\title{
Critical phenomena in complex networks
}

\author{
S. N. Dorogovtsev and A. V. Goltsev \\ Departamento de Física da Universidade de Aveiro, 3810-193 Aveiro, Portugal and \\ A.F. Ioffe Physico-Technical Institute, 194021 St. Petersburg, Russia \\ J. F. F. Mendest \\ Departamento de Física da Universidade de Aveiro, 3810-193 Aveiro, Portugal
}

\begin{abstract}
The combination of the compactness of networks, featuring small diameters, and their complex architectures results in a variety of critical effects dramatically different from those in cooperative systems on lattices. In the last few years, researchers have made important steps toward understanding the qualitatively new critical phenomena in complex networks. We review the results, concepts, and methods of this rapidly developing field. Here we mostly consider two closely related classes of these critical phenomena, namely structural phase transitions in the network architectures and transitions in cooperative models on networks as substrates. We also discuss systems where a network and interacting agents on it influence each other. We overview a wide range of critical phenomena in equilibrium and growing networks including the birth of the giant connected component, percolation, $k$-core percolation, phenomena near epidemic thresholds, condensation transitions, critical phenomena in spin models placed on networks, synchronization, and self-organized criticality effects in interacting systems on networks. We also discuss strong finite size effects in these systems and highlight open problems and perspectives.
\end{abstract}

\section{Contents}

\section{INTRODUCTION}

\section{MODELS OF COMPLEX NETWORKS}
A. Structural characteristics of networks
B. Cayley tree versus Bethe lattice
C. Equilibrium random trees versus growing ones
D. Classical random graphs
E. Uncorrelated networks with arbitrary degree distributions
1. Configuration model
2. Static model
3. Statistical mechanics of uncorrelated networks
4. Cutoffs of degree distributions
F. Equilibrium correlated networks
G. Loops in networks
H. Evolving networks
1. Preferential attachment
2. Deterministic graphs
I. Small-world networks

\section{THE BIRTH OF A GIANT COMPONENT}
A. Tree ansatz
B. Organization of uncorrelated networks
1. Evolution of the giant connected component
2. Percolation on uncorrelated networks
3. Statistics of finite connected components
4. Finite size effects
5. $k$-core architecture of networks
C. Percolation on degree-degree correlated networks
D. The role of clustering
E. Giant component in directed networks
F. Giant component in growing networks
G. Percolation on small-world networks
H. $k$-clique percolation

\section{I. $e$-core}

IV. CONDENSATION TRANSITION
A. Condensation of edges in equilibrium networks
B. Condensation of triangles in equilibrium nets
C. Condensation of edges in growing networks

\section{CRITICAL EFFECTS IN THE DISEASE} SPREADING

A. The SIS, SIR, SI, and SIRS models

B. Epidemic thresholds and prevalence

C. Evolution of epidemics

VI. THE ISING MODEL ON NETWORKS

A. Main methods for tree-like networks

1. Bethe approach

2. Belief-propagation algorithm

3. Annealed network approach

B. The Ising model on a regular tree

1. Recursion method

2. Spin correlations

3. Magnetic properties

C. The ferromagnetic Ising model on uncorrelated networks

1. Derivation of thermodynamic quantities

2. Phase transition

3. Finite-size effects

4. Ferromagnetic correlations

5. Degree-dependent interactions

D. The Ising model on small-world networks

E. Spin glass transition on networks

1. The Ising spin glass

2. The antiferromagnetic Ising model and MAX-CUT problem

3. Antiferromagnet in a magnetic field, the hard-core gas model, and vertex covers

F. The random-field Ising model

1. Phase diagram

2. Hysteresis on a fully connected graph

3. Hysteresis on a complex network

4. The random-field model at $T=0$

G. The Ising model on growing networks

1. Deterministic graphs with BKT-like transitions
${ }^{\dagger}$ Electronic address: goltsev@fis.ua.pt

‡Electronic address: jfmendes@fis.ua.pt 
2. The Ising model on growing random networks

VII. THE POTTS MODEL ON NETWORKS

A. Solution for uncorrelated networks

B. A first order transition

C. Coloring a graph

D. Extracting communities

VIII. THE XY MODEL ON NETWORKS

A. The XY model on small-world networks

B. The XY model on uncorrelated networks

IX. PHENOMENOLOGY OF CRITICAL PHENOMENA IN NETWORKS

A. Generalized Landau theory

B. Finite-size scaling

X. SYNCHRONIZATION ON NETWORKS
A. The Kuramoto model
B. Mean-field approach
C. Numerical study of the Kuramoto model
D. Coupled dynamical systems
1. Stability criterion.
2. Numerical study.

XI. SELF-ORGANIZED CRITICALITY PROBLEMS ON NETWORKS

A. Sandpiles and avalanches

B. Cascading failures

C. Congestion

XII. OTHER PROBLEMS AND APPLICATIONS
A. Contact and reaction-diffusion processes
1. Contact process
2. Reaction-diffusion processes
B. Zero-range processes
C. The voter model
D. Co-evolution models
E. Localization transitions
1. Quantum localization
2. Biased random walks
F. Decentralized search
G. Graph partitioning

XIII. SUMMARY AND OUTLOOK
A. Open problems
B. Conclusions

Acknowledgments

A. BETHE-PEIERLS APPROACH: THERMODYNAMIC PARAMETERS

B. BELIEF-PROPAGATION ALGORITHM: MAGNETIC MOMENT AND THE BETHE FREE ENERGY

\section{REPLICA TRICK}

D. MAX-CUT ON THE ERDÖS-RÉNYI GRAPH

E. EQUATIONS OF STATE OF THE POTTS MODEL ON A NETWORK

References

\section{INTRODUCTION}

By definition, complex networks are networks with more complex architectures than classical random graphs with their "simple" Poissonian distributions of connections. The great majority of real-world networks, including the World Wide Web, the Internet, basic cellular networks, and many others, are complex ones. The complex organization of these nets typically implies a skewed distribution of connections with many hubs, strong inhomogeneity and high clustering, as well as non-trivial temporal evolution. These architectures are quite compact (with a small degree of separation between vertices), infinitely dimensional, which is a fundamental property of various networks - small worlds.

Physicists intensively studied structural properties of complex networks since the end of 90's, but the current focus is essentially on cooperative systems defined on networks and on dynamic processes taking place on networks. In recent years it was revealed that the extreme compactness of networks together with their complex organization result in a wide spectrum of non-traditional critical effects and intriguing singularities. This paper reviews the progress in the understanding of the unusual critical phenomena in networked systems.

One should note that the tremendous current interest in critical effects in networks is explained not only by numerous important applications. Critical phenomena in disordered systems were among the hottest fundamental topics of condensed matter theory and statistical physics in the end of XX century. Complex networks imply a new, practically unknown in condensed matter, type of strong disorder, where fluctuations of structural characteristics of vertices (e.g., the number of neighbors) may greatly exceed their mean values. One should add to this large-scale inhomogeneity which is significant in many complex networks - statistical properties of vertices may strongly differ in different parts of a network.

The first studies of a critical phenomenon in a network were made by Solomonoff and Rapoport (1951) and Erdős and Rénvi (1959) who introduced classical random graphs and described the structural phase transition of the birth of the giant connected component. These simplest random graphs were widely used by physicists as substrates for various cooperative models.

Another basic small-world substrate in statistical mechanics and condensed matter theory is the Bethe lattice - an infinite regular tree - and its diluted variations. The Bethe lattice usually allows exact analytical treatment, and, typically, each new cooperative model is inspected on this network (as well as on the infinite fully connected graph).

Studies of critical phenomena in complex networks essentially use approaches developed for these two fundamental, related classes of networks - classical random graphs and the Bethe lattices. In these graphs and many others, small and finite loops (cycles) are rare and not essential, the architectures are locally tree-like, which is a 
great simplifying feature extensively exploited. One may say, the existing analytical and algorithmic approaches already allow one to exhaustively analyse any locally tree-like network and to describe cooperative models on it. Moreover, the tree ansatz works well even in numerous important situations for loopy and clustered networks. We will discuss in detail various techniques based on this standard approximation. It is these techniques, including, in particular, the Bethe-Peierls approximation, that are main instruments for study the critical effects in networks.

Critical phenomena in networks include a wide range of issues: structural changes in networks, the emergence of critical - scale-free - network architectures, various percolation phenomena, epidemic thresholds, phase transitions in cooperative models defined on networks, critical points of diverse optimization problems, transitions in co-evolving couples - a cooperative model and its network substrate, transitions between different regimes in processes taking place on networks, and many others. We will show that many of these critical effects are closely related and universal for different models and may be described and explained in the framework of a unified approach.

The outline of this review is as follows. In Sec. II we briefly describe basic models of complex networks. Section III contains a discussion of structural phase transitions in networks: the birth of the giant connected component of a complex random network and various related percolation problems. In Sec. IV] we describe condensation phenomena, where a finite fraction of edges, triangles, etc. are attached to a single vertex. Section $\nabla$ overviews main critical effects in the disease spreading. Sections VI, VII and VIII discuss the Ising, Potts and $X Y$ models on networks. We use the Ising model to introduce main techniques of analysis of interacting systems in networks. We place a comprehensive description of this analytical apparatus, more useful for theoretical physicists, in the Appendix. Section IX contains a general phenomenological approach to critical phenomena in networks. In Secs. $\mathrm{X}$ and $\mathrm{XI}$ we discuss specifics of synchronization and self-organized criticality on networks. Section XII briefly describes a number of other critical effects in networks. In Sec. XIII we indicate open problems and perspectives of this field. Note that for a few interesting problems, as yet uninvestigated for complex networks, we discuss only the classical random graph case.

\section{MODELS OF COMPLEX NETWORKS}

In this section we briefly introduce basic networks, which are used as substrates for models, and basic terms. For more detail see books and reviews of Albert and Barabási (2002), Dorogovtsev and Mendes (2002, 2003), Newman (2003a), Bollobás and Riordan (2003), Pastor-Satorras and Vespignani (2004), Boccaletti et al. (2006), Durrett (2006), and Caldarelli
(2007).

\section{A. Structural characteristics of networks}

A random network is a statistical ensemble, where each member - a particular configuration of vertices and edges - is realized with some prescribed probability (statistical weights). Each graph of $N$ vertices may be described by its adjacency $N \times N$ matrix $\left(a_{i j}\right)$, where $a_{i j}=0$ if edges between vertices $i$ and $j$ are absent, and $a_{i j}>0$ otherwise. In simple graphs, $a_{i j}=0,1$. In weighted networks, the adjacency matrix elements are non-negative numbers which may be non-integer - weights of edges. The simplest characteristic of a vertex in a graph is its degree $q$, that is the number of its nearest neighbors. In physics this is often called connectivity. In directed graphs, at least some of edges are directed, and one should introduce in- and out-degrees. For random networks, a vertex degree distribution $P(q)$ is the first statistical measure.

The presence of connections between the nearest neighbors of a vertex $i$ is described by its clustering coefficient $C\left(q_{i}\right) \equiv t_{i} /\left[q_{i}\left(q_{i}-1\right) / 2\right]$, where $t_{i}$ is the number of triangles (loops of length 3 ) attached to this vertex, and $q_{i}\left(q_{i}-1\right) / 2$ is the maximum possible number of such triangles. Note that in general, the mean clustering $\langle C\rangle \equiv \sum_{q} P(q) C(q)$ should not coincide with the clustering coefficient (transitivity) $C \equiv\left\langle t_{i}\right\rangle /\left\langle q_{i}\left(q_{i}-1\right) / 2\right\rangle$ which is three times the ratio of the total number of triangles in the network and the total number of connected triples of vertices. A connected triple here is a vertex with its two nearest neighbors. A triangle can be treated as a three connected triples, which explains the coefficient 3 .

A loop (simple cycle) is a closed path visiting each its vertex only once. By definition, trees are graphs without loops.

For each pair of vertices $i$ and $j$ connected by at least one path, one can introduce the shortest path length, the so-called intervertex distance $\ell_{i j}$, the corresponding number of edges in the shortest path. The distribution of intervertex distances $\mathcal{P}(\ell)$ describes the global structure of a random network, and the mean intervertex distance $\bar{\ell}(N)$ characterizes the "compactness" of a network. In finite-dimensional systems, $\bar{\ell}(N) \sim N^{1 / d}$. We, however, mostly discuss networks with the smallworld phenomenon - the so called small worlds, where $\bar{\ell}$ increases with the total number of vertices $N$ slower than any positive power, i.e., $d=\infty$ (Watts, 1999). Typically in networks, $\bar{\ell}(N) \sim \ln N$.

Another important characteristic of a vertex (or edge) is its betweenness centrality (or, which is the same, load): the number of the shortest paths between other vertices which run through this vertex (or edge). In more strict terms, the betweenness centrality $b(v)$ of vertex $v$ is defined as follows. Let $s(i, j)>0$ be the number of the shortest paths between vertices $i$ and $j$. Let $s(i, v, j)$ be the number of these paths, passing through vertex $v$. Then 

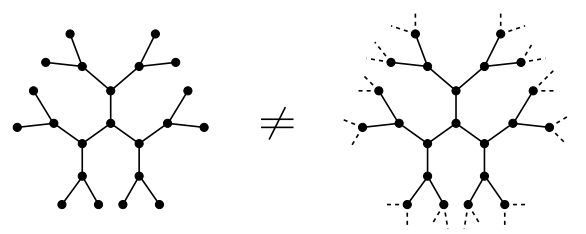

FIG. 1 The Cayley tree (on the left) versus the Bethe lattice (on the right).

$b(v) \equiv \sum_{i \neq v \neq j} s(i, v, j) / s(i, j)$. A betweenness centrality distribution is introduced for a random network.

A basic notion is a giant connected component analogous to the percolation cluster in condensed matter. This is a set of mutually reachable vertices and their interconnections, containing a finite fraction of vertices of an infinite network. Note that in physics the infinite network limit, $N \rightarrow \infty$, is also called the thermodynamic limit. The relative size of the giant component (the relative number of its vertices) and the size distribution of finite connected components describe the topology of a random network.

\section{B. Cayley tree versus Bethe lattice}

Two very different regular graphs are extensively used as substrates for cooperative models. Both are small worlds if the degree of their vertices exceeds 2 . In the (regular) Cayley tree, explained on Fig. 1, a finite fraction of vertices are dead ends. These vertices form a sharp border of this tree. There is a central vertex, equidistant from the boundary vertices. The presence of the border essentially determines the physics of interacting systems on the Cayley tree.

The Bethe lattice is an infinite regular graph (see Fig. 11). All vertices in a Bethe lattice are topologically equivalent, and boundaries are absent. Note that in the thermodynamic limit, the so called random regular graphs asymptotically approach the Bethe lattices (Johnston and Plechác,, 1998). The random regular graph is a maximally random network of vertices of equal degree. It is constructed of vertices with the same number (degree) of stubs by connecting pairs of the stubs in all possible ways.

\section{Equilibrium random trees versus growing ones}

Remarkably, random connected trees (i.e., consisting of a single connected component) may or may not be small worlds (Bialas et al., 2003; Burda et al., 2001). The equilibrium random connected trees have extremely extended architectures characterising by the fractal (Hausdorff) dimension $d_{h}=2$, i.e., $\bar{\ell}(N) \sim N^{1 / 2}$. These random trees are the statistical ensembles that consist of all possible connected trees with $N$ labelled vertices, taken

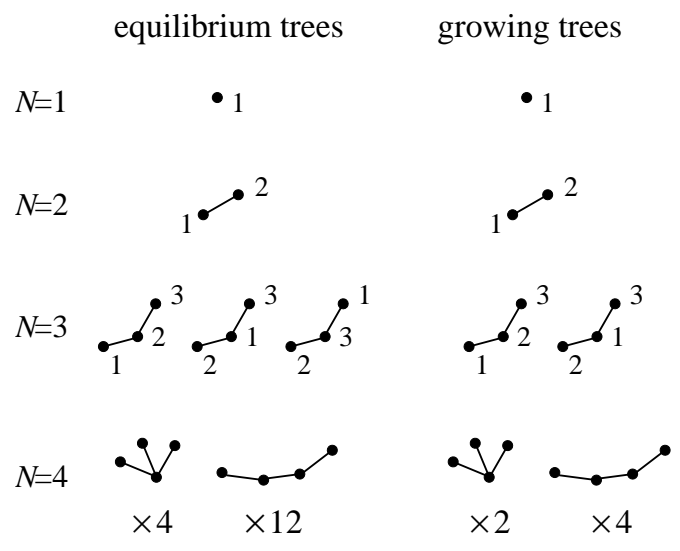

FIG. 2 Statistical ensembles of equilibrium random connected trees (left-hand side) and of growing connected trees (right-hand side) for $N=1,2,3,4$. The ensemble of equilibrium trees consists of all possible connected trees of $N$ labelled vertices, where each tree is taken with the same weight. The ensemble of growing (causal) trees is the following construction. Its members are the all possible connected trees of size $N$ that can be made by sequential attachment of new labelled vertices. Each of these trees of $N$ vertices is taken with the same weight. Notice that at $N=3$, one of the labelled graphs of the equilibrium ensemble is absent in the ensemble of growing trees. At $N=4$, we indicate the numbers of isomorphic graphs in both ensembles. (By definition, isomorphic graphs differ from each other only by vertex labels.) Already at $N=4$, the equilibrium random tree is less compact, since the probability of realization of the chain is higher in this case.

with equal probability - Fig. 2, left side. The degree distributions of these networks are rapidly decreasing, $P(q)=e^{-1} /(q-1)$ !. However one may arrive at scalefree degree distributions $P(q) \sim q^{-\gamma}$ by, for example, introducing special degree dependent statistical weights of different members of these ensembles. In this case, if $\gamma \geq 3$, then $d_{h}=2$, and if $2<\gamma<3$, then the fractal dimension is $d_{h}=(\gamma-1) /(\gamma-2)>2$.

In contrast to this, the growing (causal, recursive) random connected trees are small worlds. These trees are constructed by sequential attachment of new (labelled) vertices - Fig. 2, right side. The rule of this attachment or, alternatively, specially introduced degree dependent weights for different realizations, determine the resulting degree distributions. The mean intervertex distance in these graphs $\bar{\ell} \sim \ln N$. Thus, even with identical degree distributions, the equilibrium random trees and growing ones have quite different geometries.

\section{Classical random graphs}

Two simplest models of random networks are so close (one may say, asymptotically coincident in the thermodynamic limit) that they are together called classical random graphs. The Gilbert model, or the $G_{n p}$ model, (Gilbert, 1959; Solomonoff and Rapoport, 1951) is a ran- 
dom graph where an edge between each pair of $N$ vertices is present with a fixed probability $p$.

The slightly more difficult for analytical treatment Erdös-Rényi model (Erdös and Rénvi, 1959), which is also called the $G_{n m}$ model, is a statistical ensemble where all members - all possible graphs with a given numbers of vertices, $N$, and edges, $M$,- have equal probability of realization. The relationship between the Erdős-Rényi model and the Gilbert one is given by the following equalities for the mean degree: $\langle q\rangle=2 M / N=p N$. If $\langle q\rangle / N \rightarrow 0$ as $N \rightarrow \infty$, a network is sparse, i.e., it is far more sparse than a fully connected graph. So, the Gilbert model is sparse when $p(N \rightarrow \infty) \rightarrow 0$.

The classical random graphs are maximally random graphs under a single constraint - a fixed mean degree $\langle q\rangle$. Their degree distribution is Poissonian, $P(q)=$ $e^{-\langle q\rangle}\langle q\rangle^{q} / q$ !

\section{E. Uncorrelated networks with arbitrary degree distributions}

One should emphasize that in a random network, the degree distribution of the nearest neighbor $P_{\mathrm{nn}}(q)$ (or, which is the same, the degree distribution of an end vertex of a randomly chosen edge) does not coincide with the vertex degree distribution $P(q)$. In general random networks,

$$
P_{\mathrm{nn}}(q)=\frac{q P(q)}{\langle q\rangle}, \quad\langle q\rangle_{\mathrm{nn}}=\frac{\left\langle q^{2}\right\rangle}{\langle q\rangle}>\langle q\rangle,
$$

see Fig. 3. These simple relations play a key role in the theory of complex networks.

By definition, in uncorrelated networks correlations are absent, in particular, there are no correlations between degrees of the nearest neighbors. That is, the joint distribution of degrees of the nearest neighbor vertices factors into the product:

$$
P\left(q, q^{\prime}\right)=\frac{q P(q) q^{\prime} P\left(q^{\prime}\right)}{\langle q\rangle^{2}} .
$$

Thus, the architectures of uncorrelated networks are determined by their degree distributions. The ErdősRényi and Gilbert models are simple uncorrelated networks. Below we list the models of complex uncorrelated

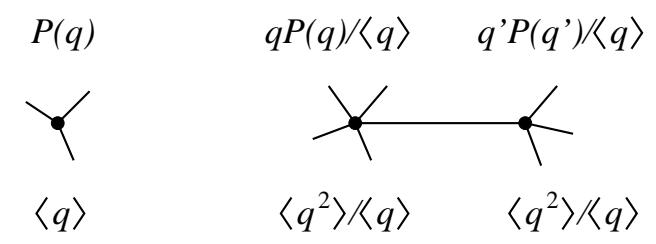

FIG. 3 The distribution of connections and the mean degree of a randomly chosen vertex (on the left) differ sharply from those of end vertices of a randomly chosen edge (on the right). networks, which are actually very close to each other in the thermodynamic limit. In this limit all these networks are locally tree-like (if they are sparse, of course), with only infinite loops.

\section{Configuration model}

The direct generalization of the Erdős-Rényi graphs is the famous configuration model formulated by Bollobás (1980), see also the work of Bender and Canfield (1978). In graph theory, these networks are also called random labelled graphs with a given degree sequence. The configuration model is the statistical ensemble, whose members are realized with equal probability. These members are all possible graphs with a given set $\left\{N_{q}=N P(q)\right\}$, $q=0,1,2,3, \ldots$, where $N_{q}$ is the number of vertices of degree $q$. In simple terms, the configuration model provides maximally random graphs with a given degree distribution $P(q)$.

This construction may be also portrayed in more graphic terms: (i) Attach stubs - edge-halves - to $N$ vertices according to a given sequence of numbers $\left\{N_{q}\right\}$. (ii) Pair randomly chosen stubs together into edges. Since stubs of the same vertex may be paired together, the configuration model, in principle, allows a number of loops of length one as well as multiple connections. Fortunately, these may be neglected in many problems.

Using relation (11) gives the formula $z_{2}=\left\langle q^{2}\right\rangle-\langle q\rangle$ for the mean number of the second nearest neighbors of a vertex. That is, the mean branching coefficient of the configuration model and, generally, of an uncorrelated network is

$$
B=\frac{z_{2}}{z_{1}}=\frac{\left\langle q^{2}\right\rangle-\langle q\rangle}{\langle q\rangle}
$$

where $z_{1}=\langle q\rangle$. Consequently, the mean number of the $\ell$ th nearest neighbors of a vertex is $z_{\ell}=z_{1}\left(z_{2} / z_{1}\right)^{\ell-1}$. So the mean intervertex distance is $\bar{\ell}(N) \cong \ln N / \ln \left(z_{2} / z_{1}\right)$ (Newman et al., 2001).

The distribution of the intervertex distances in the configuration model is quite narrow. Its relative width approaches zero in the thermodynamic limit. In other words, in this limit, almost all vertices of the configuration model are mutually equidistant (Dorogovtsev et al., 2003a). We emphasise that this remarkable property is valid for a very wide class of networks with the smallworld phenomenon.

The configuration model was generalized to bipartite networks (Newman et al., 2001). By definition, a bipartite graph contains two kinds of vertices, and only vertices of different kinds may be interlinked. In short, the configuration model of a bipartite network is a maximally random bipartite graph with two given degree distributions for two types of vertices. 


\section{Static model}

The direct generalization of the Gilbert model is the static one (Goh et al., 2001), see also works of Chung and Lu (2002), Soderberg (2002), and Caldarelli et al. (2002). These are graphs with a given sequence of desired degrees. These desired degrees $\left\{d_{i}\right\}$ play role of "hidden variables" defined on vertices $i=$ $1,2, \ldots, N$. Pairs of vertices $(i j)$ are connected with probabilities $p_{i j}=1-\exp \left(-d_{i} d_{j} / N\langle d\rangle\right)$. The degree distribution of resulting network $P(q)$ tends to a given distribution of desired degrees at sufficiently large $q$. It is important that at small enough $d_{i}$, the probability $p_{i j} \cong d_{i} d_{j} /(N\langle d\rangle)$. The exponential function keeps the probability below 1 even if $d_{i} d_{j}>N\langle d\rangle$ which is possible if the desired degree distribution is heavy tailed.

\section{Statistical mechanics of uncorrelated networks}

It is also easy to generate random networks by using a standard thermodynamic approach, see Burda et al. (2001), Bauer and Bernard (2002), and Dorogovtsev et al. (2003b). In particular, assuming that the number of vertices is constant, one may introduce "thermal" hopping of edges or their rewiring. These processes lead to relaxational dynamics in the system of edges connecting vertices. The final state of this relaxation process - an equilibrium statistical ensemblemay be treated as an "equilibrium random network". This network is uncorrelated if the rate/probability of rewiring depends only on degrees of host vertices and on degrees of targets, and, in addition, if rewirings are independent. The resulting diverse degree distributions are determined by two factors: a specific degree dependent rewiring and the mean vertex degree in the network. Note that if multiple connections are allowed, this construction is essentially equivalent to the simple balls-inboxes (or backgammon) model (Bialas et al., 2000, 1997), where ends of edges - balls - are statistically distributed among vertices - boxes.

\section{Cutoffs of degree distributions}

Heavy tailed degree distributions $P(q)=\langle N(q)\rangle / N$ in finite networks, inevitably end by a rapid drop at large degrees - cutoff. Here, $\langle N(q)\rangle$ is the number of vertices of degree $q$ in a random network, averaged over all members of the corresponding statistical ensemble. The knowledge of the size dependence of the cutoff position, $q_{\text {cut }}(N)$ is critically important for the estimation of various size effects in complex networks. The difficulty is that the form of $q_{\text {cut }}(N)$ is highly model dependent.

We here present estimates of $q_{\text {cut }}(N)$ in uncorrelated scale-free networks, where $P(q) \sim q^{-\gamma}$. The results essentially depend on (i) whether exponent $\gamma$ is above or below 3, and (ii) whether multiple connections are al- lowed in the network or not.

In the range $\gamma \geq 3$, the resulting estimates are the same in networks with multiple connections (Burda et al., 2001) and without them (Dorogovtsev et al., 2005). In this range, strict calculation of a degree distribution taking into account all members of a statistical network ensemble leads to $q_{\text {cut }}(N) \sim N^{1 / 2}$. The total number of the members of an equilibrium network ensemble (e.g., for the configuration model) is huge, say, of the order of $N$ !. However, in empirical research or simulations, ensembles under investigation have rather small number $n$ of members - a whole ensemble may consist of a single empirically studied map or of a few runs in a simulation. Often, only a single network configuration is used as a substrate in simulations of a cooperative model. In these measurements, a natural cutoff of an observed degree distribution arises (Cohen et al., 2000; Dorogovtsev et al., 2001c). Its degree, much lower than $N^{1 / 2}$, is estimated from the following condition. In the $n$ studied ensemble members, a vertex degree exceeding $q_{\text {cut }}$ should occur one time: $n N \int_{q_{\text {cut }}(N)}^{\infty} d q P(q) \sim 1$. This gives the really observable cutoff:

$$
q_{\text {cut }}(N, \gamma \geq 3) \sim(n N)^{1 /(\gamma-1)}
$$

if $n \ll N^{(\gamma-3) / 2}$, which is a typical situation, and $q_{\text {cut }}(N, \gamma \geq 3) \sim N^{1 / 2}$ otherwise.

In the interesting range $2<\gamma<3$, the cutoff essentially depends on the kind of an uncorrelated network. If in an uncorrelated network, multiple connections are allowed, then $q_{\text {cut }}(N, 2<\gamma<3) \sim$ $N^{1 /(\gamma-1)}$. In uncorrelated networks without multiple connections, $q_{\text {cut }}(N, 2<\gamma<3) \sim N^{1 / 2} \ll$ $N^{1 /(\gamma-1)}$ (Burda and Krzywicki, 2003), although see Dorogovtsev et al. (2005) for a different estimate for a specific model without multiple connections. For discussion of the cutoff problem in the static model in this range of exponent $\gamma$, see Lee et al. (2006a).

Seyed-allaei et al. (2006) found that in scale-free uncorrelated networks with exponent $\gamma<2$, the cutoff is $q_{\text {cut }}(N, 1<\gamma<2) \sim N^{1 / \gamma}$. They showed that the mean degree of these networks increases with $N$ : namely, $\langle q\rangle \sim N^{(2-\gamma) / \gamma}$.

For the sake of completeness, we here mention that in growing scale-free recursive networks, $q_{\text {cut }}(N, \gamma>2) \sim N^{1 /(\gamma-1)}$ (Dorogovtsev et al., 2001c; Krapivsky and Redner, 2002; Waclaw and Sokolov, 2007). Note that the growing networks are surely correlated.

\section{F. Equilibrium correlated networks}

The simplest kind of correlations in a network are correlations between degrees of the nearest neighbor vertices. These correlations are described by the joint degree-degree distribution $P\left(q, q^{\prime}\right)$. If $P\left(q, q^{\prime}\right)$ is not factorized, unlike equality (2), the network is correlated (Maslov and Sneppen, 2002; Newman, 2002b). 
The natural generalization of uncorrelated networks, which is still sometimes analytically treatable, are networks maximally random under the constraint that their joint degree-degree distributions $P\left(q, q^{\prime}\right)$ are fixed. That is, only these correlations are present. In the hierarchy of equilibrium network models, this is the next, higher, level, after the classical random graphs and uncorrelated networks with an arbitrary degree distribution. Note that networks with this kind of correlations are still locally tree-like in the sparse network regime. In this sense they may be treated as random Bethe lattices.

These networks may be constructed in the spirit of the configuration model. An alternative constructionnetworks with hidden variables - directly generalizes the static model. These are networks, where (i) a random hidden variable $h_{i}$ with distribution $P_{h}(h)$ is assigned to each vertex, and (ii) each pair of vertices $(i j)$ is connected by an edge with probability $p\left(h_{i}, h_{j}\right)$ (Boguñá and Pastor-Satorras, 2003; Caldarelli et al., 2002; Soderberg, 2002). The resulting joint degree-degree distribution is determined by $P_{h}(h)$ and $p\left(h, h^{\prime}\right)$ functions.

\section{G. Loops in networks}

The above-described equilibrium network models share the convenient locally tree-like structure in the sparse network regime. The number of loops $\mathcal{N}_{L}$ of length $L$ in a network allows us to quantify this important property. We stress that the total number of loops in these networks is in fact very large. Indeed, the typical intervertex distance $\sim \ln N$, so that the number of loops with lengths $\gtrsim \ln N$ should be huge. On the other hand, there is few loops of smaller lengths. In simple terms, if the second moment of the degree distribution is finite in the thermodynamic limit, then the number of loops of any given finite length is finite even in an infinite network. Consequently, the probability that a finite loop passes through a vertex is quite small, which explains the treelikeness.

In more precise terms, the number of loops in uncorrelated undirected networks is given by the following expression (Bianconi and Capocci, 2003; Bianconi and Marsili, 2005a):

$$
\mathcal{N}_{L} \sim \frac{1}{2 L}\left(\frac{\left\langle q^{2}\right\rangle-\langle q\rangle}{\langle q\rangle}\right)^{L},
$$

which is valid for sufficiently short (at least, for finite) loops, so that the clustering coefficient $C(k)=C=$ $\langle C\rangle=\left(\left\langle q^{2}\right\rangle-\langle q\rangle\right)^{2} /\left(N\langle q\rangle^{3}\right)$ (Newman, 2003b). In addition, there are exponentially many, $\ln \mathcal{N}_{L} \propto N$, loops of essentially longer lengths (roughly speaking, longer than the network diameter). These "infinite loops", as they are longer than a correlation length for a cooperative system, do not violate the validity of the tree approximation. Moreover, without these loops - in perfect trees - phase transitions are often impossible, as, e.g., in the Ising model on a tree. The mean number of loops of length $L$ passing through a vertex of degree $k$ is $\mathcal{N}_{L}(k) \approx[k(k-1) /(\langle q\rangle N)][(L-1) / L] \mathcal{N}_{L-1}$. With degree distribution cutoffs represented in Sec. II.E.4 formula (5) leads to finite $\mathcal{N}_{L}$ in uncorrelated networks with $\gamma>3$, and to a large number of loops

$$
\mathcal{N}_{L} \sim \frac{1}{2 L}(a /\langle q\rangle)^{L} N^{L(3-\gamma) / 2},
$$

for $2<\gamma<3$ and $\left\langle q^{2}\right\rangle \cong a N^{(3-\gamma) / 2}$, where $a$ is a constant. For the statistics of loops in directed networks, see Bianconi et al. (2007). Note that formulas (5) and (6) indicate that even the sparse uncorrelated networks are actually loopy if $\gamma<3$. Nonetheless, we suppose that the tree ansatz still works even in this situation (see discussion in following sections).

\section{H. Evolving networks}

Self-organization of non-equilibrium networks during their evolution (usually growth) is one of traditional explanations of network architectures with a great role of highly connected hubs. One should also stress that nonequilibrium networks inevitably have a wide spectrum of correlations.

The simplest random growing network is a random recursive tree defined as follows. The evolution starts from a single vertex. At each time step, a new vertex is attached to a random existing one by an edge. The resulting random tree has an exponential degree distribution.

\section{Preferential attachment}

To arrive at a heavy-tailed degree distribution, one may use preferential attachment - vertices for linking are chosen with probability proportional to a special function $f(q)$ of their degrees (preference function). In particular, the scale-free networks are generated with a linear preference function.

A recursive network growing by the following way is rather representative. The growth starts with some initial configuration, and at each time step, a new vertex is attached to preferentially chosen $m \geq 1$ existing vertices by $m$ edges. Each vertex for attachment is chosen with probability, proportional to a linear function of its degree, $q+A$, where the constant $A>-m$. In particular, if $A=0$ - the proportional preference,- this is the BarabásiAlbert model (Barabási and Albert, 1999), where the $\gamma$ exponent of the degree distribution is equal to 3 . In general, for a linear preferential attachment, the degree distribution exponent is $\gamma=3+A / m$ (Dorogovtsev et al., 2000; Krapivsky et al., 2000).

Among these recursive networks, the Barabási-Albert model is a very special case: it has anomalously weak degree-degree correlations for the nearest neighbors, and so it is frequently treated as "almost uncorrelated". 
(a)

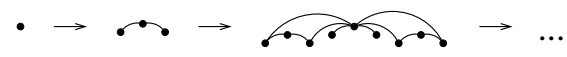

(b)

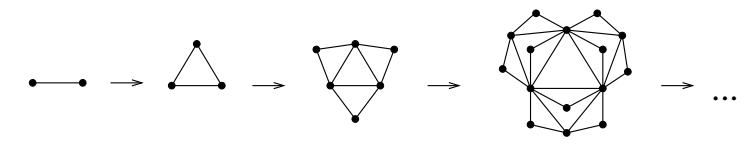

(c)

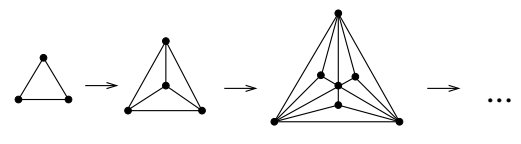

(d)

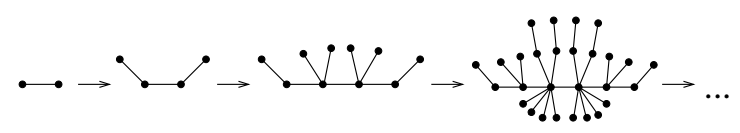

FIG. 4 Examples of deterministic small worlds: (a) of Barabási et al. (2001), (b) of Dorogovtsev and Mendes (2002) and Dorogovtsev et al. (2002a), (c) of Andrade et al. (2005) and Dove and Massen (2005), (d) of Jung et al. (2002). The $\gamma$ exponent for each of these four deterministic graphs equals $1+\ln 3 / \ln 2=2.585 \ldots$

The idea of preferential attachment providing complex network architectures was well explored. The smooth variations of these diverse structures with various model parameters were extensively studied. For example, Szabó et al. (2003) described the variations of the degree-dependent clustering in simple generalizations of the Barabási-Albert model.

\section{Deterministic graphs}

Deterministic graphs often provide the only possibility for analytical treatment of difficult problems. Moreover, by using these graphs, one may mimic complex random networks surprisingly well. Fig. 4 demonstrates a few simple "scale-free" deterministic graphs, which show the small-world phenomenon and whose discrete degree distribution have a power-law envelope.

\section{Small-world networks}

The small-world networks introduced by Watts and Strogatz (1998) are superpositions of finite dimensional lattices and classical random graphs, thus combining their properties. One of variations of the Watts-Strogatz model is explained in Fig. 5; randomly chosen pairs of vertices in a one-dimensional lattice are connected by shortcuts. There is a smooth crossover from a lattice to a small-world geometry with an increasing number of shortcuts. Remarkably, even with extremely low relative numbers of shortcuts, these networks demonstrate the small-world phenomenon.

Kleinberg (1999, 2000) used an important generalization of the Watts-Strogatz model. In the Kleinberg network ("the grid-based model with exponent $\alpha$ "), the

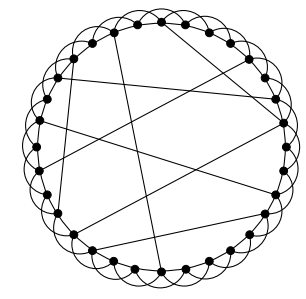

FIG. 5 A simple variation of the Watts-Strogatz model (Watts, 1999; Watts and Strogatz, 1998). Adapted from Newman (2000).

probability that a shortcut connects a pair of vertices separated by Euclidean distance $r$ decreases as $r^{-\alpha}$. The resulting network geometry critically depends on the value of exponent $\alpha$.

We end this section with a short remark. In solid state physics, boundary conditions play an important role. We stress that as a rule, the networks under discussion have no borders. So the question of boundary conditions is meaningless here. There are very few exceptions, e.g., the Cayley tree.

\section{THE BIRTH OF A GIANT COMPONENT}

This is a basic structural transition in the network architecture. Numerous critical phenomena in cooperative models on networks can be explained by taking into account the specifics of this transition in complex networks. The birth of a giant connected component corresponds to the percolation threshold notion in condensed matter. The study of random graphs was started with the discovery and description of this transition (Erdös and Rénvi, 1959; Solomonoff and Rapoport, 1951). Remarkably, it takes place in sparse networks, at $\langle q\rangle \sim$ const, which makes this range of mean degrees most interesting.

\section{A. Tree ansatz}

The great majority of analytical results for cooperative models on complex networks were obtained in the framework of the tree approximation. This ansatz assumes the absence of finite loops in a network in the thermodynamic limit and allows only infinite loops. The allowance of the infinite loops is of primary importance since they greatly influence the critical behavior. Indeed, without loops, that is on perfect trees, the ferromagnetic order, say, in the Ising model occurs only at zero temperature. Also, the removal of even a vanishingly small fraction of vertices or edges from a perfect tree eliminates the giant connected component.

The tree ansatz allows one to use the convenient techniques of the theory of random branching processes. On the other hand, in the framework of this ansatz, equilibrium networks are actually equivalent to random Bethe 
(a)

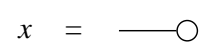

(b)

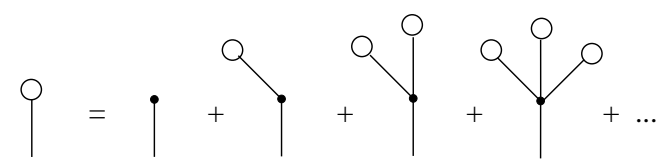

(c)

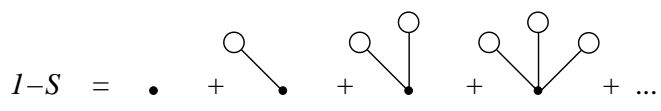

FIG. 6 (a) The graphic notation for the probability $x$ that, following a randomly chosen edge to one of its end vertices, we arrive at a finite connected component. (b) Equation (8) or, equivalently, Eq. (10) in graphic form. (c) The graphic representation of formula (9) and of equivalent relation (11) for the relative size $S$ of the giant connected component.

lattices.

\section{B. Organization of uncorrelated networks}

The mathematical solution of the problem of organization of arbitrary uncorrelated networks as a system of connected components was proposed by Mollov and Reed (1995, 1998). In the works of Newman et al. (2001) and of Callaway et al. (2000) these ideas were represented and developed using the apparatus and language of physics. Here we describe these fundamental results and ideas in simple terms. The reader may refer to the papers of Newman et al. (2001) and Newman (2003b) for the details of this theory based on the generating function technique.

\section{Evolution of the giant connected component}

The theory of uncorrelated networks (we mostly discuss the configuration model, which is completely described by the degree distribution $P(q)$ and size $N$ ) is based on their following simplifying features:

(i) The sole characteristic of a vertex in these networks is its degree, in any other respect, the vertices are statistically equivalent - there are no borders or centers, or older or younger vertices in these models. The same is valid for edges.

(ii) The tree ansatz is supposed to be valid.

(iii) Formulas (1) and (2) are valid (see Fig. 33).

Feature (i) allows one to introduce the probability $x$ that, following a randomly chosen edge to one of its end vertices, he or she arrives at a finite connected component. In more strict terms, choose a random edge; choose its random end; then $x$ is the probability that after removing this edge, the chosen end vertex will belong to a finite connected component. A graphic representation of $x$ is introduced in Fig. 6(a). The probability that an edge belongs to one of finite components is, graphically,

$$
\bigcirc-\bigcirc=x^{2} .
$$

This is the probability that following an edge in any direction, we arrive at finite trees. Thus $1-x^{2}$ is a fraction of edges which are in the giant connected component. This simple relation enables us to measure $x$. Using features (i), (ii), and (iii) immediately leads to the following self-consistent equation for $x$ and expression for the probability $1-S$ that a vertex belongs to a finite connected component:

$$
\begin{aligned}
& x=\sum_{q} \frac{q P(q)}{\langle q\rangle} x^{q-1}, \\
& 1-S=\sum_{q} P(q) x^{q} .
\end{aligned}
$$

In particular, relation (9) is explained as follows. A vertex belongs to a finite connected component if and only if following every its edge in direction from this vertex we arrive at a finite tree. The probability of this event is $x^{q}$ for a vertex of degree $q$. For a randomly chosen vertex, we must sum over $q$ the products of $x^{q}$ and the probability $P(q)$. One can see that $S$ is the relative size of the giant connected component. Figures 6(b) and (c) present these formulas in graphic form and explain them. Note that if $P(q=0,1)=0$, then Eq. (8) has the only solution $x=1$, and so $S=1$, i.e., the giant connected component coincides with the network. Using the generating function of the degree distribution, $\phi(z) \equiv \sum_{q} P(k) z^{q}$ and the notation $\phi_{1}(z) \equiv \phi^{\prime}(z) / \phi^{\prime}(1)=\phi^{\prime}(z) /\langle q\rangle$ gives

$$
\begin{aligned}
& x=\phi_{1}(x), \\
& S=1-\phi(x) .
\end{aligned}
$$

These relations demonstrate the usefulness of the generating function technique in network theory. The deviation $1-x$ plays the role of the order parameter. If Eq. (8) has a non-trivial solution $x<1$, then the network has the giant connected component. The size of this component can be found by substituting the solution of Eqs. (8) or (10) into formulas (9) or (11). Remarkably, the resulting $S$ is obtained by only considering finite connected components [which are (almost) surely trees in these networks], see Fig. 6. Knowing the size of the giant connected component and the total number of finite components, one can find the number of loops in the giant component. For the calculation of this number, see Lee et al. (2004c). Applying generating function techniques in a similar way one may also describe the organization of connected components in the bipartite uncorrelated networks, see, e.g., Soderberg (2002). 
The analysis of Eq. (8) shows that an uncorrelated network has a giant connected component when the mean number of second nearest neighbors of a randomly chosen vertex $z_{2}=\left\langle q^{2}\right\rangle-\langle q\rangle$ exceeds the mean number of nearest neighbors: $z_{2}>z_{1}$. This is the Molloy-Reed criterion:

$$
\left\langle q^{2}\right\rangle-2\langle q\rangle>0
$$

(Molloy and Reed, 1995). For the Poisson degree distribution, i.e., for the classical random graphs, $z_{2}=\langle q\rangle^{2}$, and so the birth point of the giant connected component is $z_{1}=1$. In the Gilbert model, this corresponds to the critical probability $p_{c}(N \rightarrow \infty) \cong 1 / N$ that a pair vertices is connected. These relations explain the importance of the sparse network regime, where this transition takes place. The Molloy-Reed criterion shows that the divergence of the second moment of the degree distribution guarantees the presence of the giant connected component.

Exactly at the birth point of the giant connected component, the mean size of a finite component to which a randomly chosen vertex belongs diverges as follows:

$$
\langle s\rangle=\frac{\langle q\rangle^{2}}{2\langle q\rangle-\left\langle q^{2}\right\rangle}+1
$$

Newman et al. (2001). This formula is given for the phase without the giant connected component. In this problem, $\langle s\rangle$ plays the role of susceptibility. Usually, it is convenient to express the variation of the giant component near the critical point and other critical properties in terms of the deviation of one parameter, e.g., the mean degree $\langle q\rangle$, from its critical value, $\langle q\rangle_{c}$. Usually, the resulting singularities in terms of $\langle q\rangle-\langle q\rangle_{c}$ are the same as in terms of $p-p_{c}$ in the percolation problem on complex networks ( $p$ is the concentration of undeleted vertices, see below). Note that in scale-free networks with fixed exponent $\gamma$ one may vary the mean degree by changing the low degree part of a degree distribution.

\section{Percolation on uncorrelated networks}

What happens with a network if a random fraction $1-p$ of its vertices (or edges) are removed? In this site (or bond) percolation problem, the giant connected component plays the role of the percolation cluster which may be destroyed by decreasing $p$. Two equivalent approaches to this problem are possible. The first way (Cohen et al., 2000) uses the following idea. (i) Find the degree distribution of the damaged network, which is $\tilde{P}(q)=\sum_{r=q}^{\infty} P(r) C_{q}^{r} p^{q}(1-p)^{r-q}$ both for the site and bond percolation. (ii) Since the damaged network is obviously still uncorrelated, Eqs. (8) and (9) with this $\tilde{P}(q)$ describe the percolation.

The second way is technically more convenient: derive direct generalizations of Eqs. (8) and (9) with the parameter $p$ and the degree distribution $P(q)$ of the original, undamaged network (Callawav et al., 2000). Simple

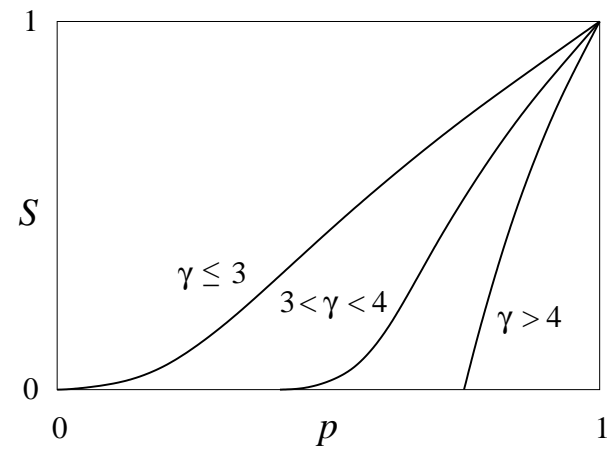

FIG. 7 The effect of the heavy-tailed architecture of a network on the variation of its giant connected component under random damage. The relative size of the giant connected component, $S$, is shown as a function of the concentration $p$ of the retained vertices in the infinite network.

arguments, similar to those illustrated by Fig. 6, immediately lead to

$$
\begin{aligned}
& x=1-p+p \sum_{q} \frac{q P(q)}{\langle q\rangle} x^{q-1}, \\
& 1-S=1-p+p \sum_{q} P(q) x^{q} .
\end{aligned}
$$

Although Eq. (14) is valid for both the site and bond percolation, relation (15) is valid only for site percolation. For the bond percolation problem, use Eq. (9). One can see that the giant connected component is present when

$$
p z_{2}>z_{1}
$$

that is, the percolation threshold is at

$$
p_{c}=\frac{z_{1}}{z_{2}}=\frac{\langle q\rangle}{\left\langle q^{2}\right\rangle-\langle q\rangle},
$$

So, in particular, $p_{c}=1 /\langle q\rangle$ for classical random graphs, and $p_{c}=1 /(q-1)$ for random regular graphs. Relations (16) and (17) show that it is practically impossible to eliminate the giant connected component in an infinite uncorrelated network if the second moment of its degree distribution diverges - the network is ultraresilient against random damage or failures (Albert et al., 2000; Cohen et al., 2000). In scale-free networks, this takes place if $\gamma \leq 3$. Callaway et al. (2000) considered a more general problem, where the probability $p(q)$ that a vertex is removed depends on its degree. As is natural, the removal of highly connected hubs from a scale-free network - an intentional damage effectively destroys its giant connected component (Albert et al., 2000; Cohen et al., 2001).

Near the critical point, the right hand side of Eq. (14) for the order parameter $1-x$ becomes non-analytic if the higher moments of the degree distribution diverge. This leads to unusual critical singularities in these percolation 
problems and, more generally, to unusual critical phenomena at the birth point of the giant connected component in networks with heavy-tailed degree distributions (Cohen et al., 2002, 2003a). For the sake of convenience, let the infinite uncorrelated network be scale-free. In this case, the critical behavior of the size $S$ of the giant connected component is as follows (Cohen et al., 2002):

(i) if $\gamma>4$, i.e., $\left\langle q^{3}\right\rangle<\infty$, then $S \propto p-p_{c}$, which is the standard mean-field result, also valid for classical random graphs;

(ii) if $3<\gamma<4$, then $S \propto\left(p-p_{c}\right)^{1 /(\gamma-3)}$, i.e., the $\beta$ exponent equals $1 /(\gamma-3)$;

(iii) if $\gamma=3$, then $p_{c}=0$ and $S \propto p \exp [-2 /(p\langle q\rangle)]$;

(iv) if $2<\gamma<3$, then $p_{c}=0$ and $S \propto p^{1 /(3-\gamma)}$.

These results are schematically shown in Fig. 7. We stress that the unusual critical exponents here are only the consequence of a fat-tailed degree distribution, and the theory is essentially of mean-field nature. Note that we discuss only unweighted networks, where edges have unit weights. For percolation on weighted networks, see Braunstein et al. (2003a, 2004); Li et al. (2007) and references therein. In weighted networks one can naturally introduce a mean length of the path along edges with the minimum sum of weights, $\bar{\ell}_{\mathrm{opt}}$. Based on the percolation theory Braunstein et al. showed that in the Erdős-Rényi graphs with a wide weight distribution, the optimal path length $\bar{\ell}_{\text {opt }} \sim N^{1 / 3}$.

Numerous variations of percolation on networks may be considered. In particular, one may remove vertices from a network with a degree-dependent probability (Albert et al., 2000; Callawav et al., 2000; Gallos et al., 2005).

The probability that a vertex of degree $q$ belongs to the giant connected component is $1-x^{q}$ [compare with Eq (9)], so that it is high for highly connected vertices. Here $x$ is the physical root of Eq. (8) for the order parameter. The degree distribution of vertices in the giant connected component (GCC) is

$$
P_{\mathrm{GCC}}(q)=\frac{P(q)\left(1-x^{q}\right)}{1-\sum_{q} P(q) x^{q}} .
$$

Therefore at the birth point $(x \rightarrow 1)$ of the giant connected component, the degree distribution of its vertices is proportional to $q P(q)$. Thus, in networks with slowly decreasing degree distributions, the giant connected component near its birth point mostly consists of vertices with high degrees.

Cohen et al. (2001, 2003a) found that at the birth point, the giant connected component does not have a small-world geometry (that is, with a diameter growing with the number of vertices $N$ slower than any positive power of $N$ ) but a fractal one. Its fractal dimension-a chemical dimension $d_{l}$ in their notations - equals $d_{l}(\gamma>$ $4)=2$ and $d_{l}(3<\gamma<4)=(\gamma-2) /(\gamma-3)$. That is, the mean intervertex distance in the giant connected component (of size $n$ ) at the point of its disappearance is quite large, $\bar{\ell} \sim n^{d_{l}}$. To be clear, suppose that we are destroying a small world by deleting its vertices. Then precisely at the moment of destruction, a tiny remnant of the network has a much greater diameter than the original compact network. It is important that this remnant is an equilibrium tree with a degree distribution characterized by exponent $\gamma-1$. Indeed, recall that in Sec. II.C we indicated that equilibrium connected trees have a fractal structure. So substituting $\gamma-1$ for $\gamma$ in the expression for the fractal dimension of equilibrium connected trees Burda et al. (2001), see Sec. II.C, we readily explain the form of $d_{l}(\gamma)$.

\section{Statistics of finite connected components}

The sizes of largest connected components $s^{(i)}$ depend on the number of vertices in a network, $N$. Here the index $i=1$ is for the largest component, $i=2$ is for the second largest component, and so on. In the classical random graphs, $s^{(i)}(N)$ with a fixed $i$ and $N \rightarrow \infty$ are as follows (for more detail see the graph theory papers of Borgs et al. (2001) and of Bollobás and Riordan (2003)):

(i) for $p<p_{c}\left(1-C N^{-1 / 3}\right), \quad s^{(i \geq 1)}(N) \sim \ln N$;

(ii) within the so called scaling window $\left|p-p_{c}\right|<$ $C N^{-1 / 3}, \quad s^{(i \geq 1)}(N) \sim N^{2 / 3}$

(iii) for $p>p_{c}\left(1+C N^{-1 / 3}\right), s^{(1)}(N) \sim N, s^{(i>1)}(N) \sim$ $\ln N$ (Bollobás, 1984).

Here $C$ denotes corresponding constants and $p=\langle q\rangle / N$.

In Sec. IX.B we will present a general phenomenological approach to finite-size scaling in complex networks. The application of this approach to scale-free networks with degree distribution exponent $\gamma$ allows one to describe the sizes of the largest connected components:

(i) if $\gamma>4$, the same formulas hold, as for the classical random graphs;

(ii) if $3<\gamma<4$, then $s^{(i \geq 1)}(N) \sim N^{(\gamma-2) /(\gamma-1)}$ within the scaling window $\left|p-p_{c}\right|<C N^{-(\gamma-3) /(\gamma-1)}$ (Kalisky and Cohen, 2006), and the classical results, represented above, hold outside of the scaling window.

Similarly, one can write

$$
p_{c}(N=\infty)-p_{c}(N) \sim N^{-(\gamma-3) /(\gamma-1)}
$$

for the deviation of the percolation threshold in the range $3<\gamma<4$. (Note that rigorously speaking, $p_{c}$ is well defined only in the $N \rightarrow \infty$ limit.) We will discuss the size effect in networks with $2<\gamma<3$ in Sec. III.B.4.

Let us compare these results with the corresponding formulas for the standard percolation on lattices. If the 
dimension of a lattice is below the upper critical dimension for the percolation problem, $d<d_{u}=6$, then

$$
s^{(i \geq 1)}(N) \sim N^{d_{f} / d}
$$

within the scaling window $\left|p-p_{c}\right|<$ const $N^{-1 /(\nu d)}$. Here $d_{f}=(d+2-\eta) / 2=\beta / \nu+2-\eta$ is the fractal dimension of the percolation cluster in the critical point measured in the $d$-dimensional space by using a box counting procedure, $\nu$ is the correlation length exponent, and $\eta$ is the Fisher exponent. (The boxes in this box counting procedure are based on an original, undamaged network.) Above the upper critical dimension, which is the case for the small worlds, one must replace, as is usual, $d$ in these formulas (and in scaling relations) by $d_{u}$ and substitute the mean-field values of the critical exponents $\nu, \eta$, and $\beta$. Namely, use $\nu=1 / 2$ and $\eta=0$. For networks, the meanfield exponent $\beta=\beta(\gamma)$, and so, similarly to Hong et al. (2007a), we may formally introduce the upper critical dimension $d_{u}(\gamma)=2 \beta / \nu+2-\eta=4 \beta(\gamma)+2$ and the fractal dimension $d_{f}(\gamma)=\beta / \nu+2-\eta=2 \beta(\gamma)+2$.

With the known order parameter exponent $\beta(\gamma)$ from Sec. III.B.2 this heuristic approach gives the fractal dimension

$$
d_{f}(\gamma \geq 4)=4 \text { and } d_{f}(3<\gamma<4)=2 \frac{\gamma-2}{\gamma-3}
$$

(Cohen et al., 2003a). Note that this fractal dimension $d_{f}$ does not coincide with the "chemical dimension" $d_{l}$ discussed above but rather $d_{f}=2 d_{l}$. Similarly,

$$
d_{u}(\gamma \geq 4)=6 \text { and } d_{u}(3<\gamma<4)=2 \frac{\gamma-1}{\gamma-3}
$$

(Cohen et al., 2003a; Hong et al., 2007a; Wu et al., 2007a). With these $d_{u}(\gamma)$ and $d_{f}(\gamma)$, we reproduce the above formulas for finite-size networks.

The distribution of sizes of connected components in the configuration model was derived by using the generating function technique (Newman, 2007; Newman et al., 2001). Let $\mathcal{P}(s)$ be the size distribution of a finite component to which a randomly chosen vertex belongs and $\mathcal{Q}(s)$ be the distribution of the total number of vertices reachable following a randomly chosen edge. $h(z) \equiv$ $\sum_{s} \mathcal{P}(s) z^{s}$ and $h_{1}(z) \equiv \sum_{s} \mathcal{Q}(s) z^{s}$ are the corresponding generating functions. Then

$$
\begin{aligned}
& h(z)=z \phi\left(h_{1}(z)\right), \\
& h_{1}(z)=z \phi_{1}\left(h_{1}(z)\right)
\end{aligned}
$$

(Newman et al., 2001). To get $h(z)$ and its inverse transformation $\mathcal{P}(s)$, one should substitute the solution of Eq. (24) into relation (23).

Equations (23), (24) have an interesting consequence for scale-free networks without a giant connected component. If the degree distribution exponent is $\gamma>3$, then in this situation the size distribution $\mathcal{P}(s)$ is also asymptotically power-law, $\mathcal{P}(s) \sim s^{-(\gamma-1)}$ (Newman, 2007). To arrive at this result, one must recall that if a function is power-law, $P(k) \sim k^{-\gamma}$, then its generating function near $z=1$ is $\phi(z)=a(z)+C(1-z)^{\gamma-1}$, where $a(z)$ is some function, analytic at $z=1$ and $C$ is a constant. Substituting this $\phi(z)$ into Eqs. (23) and (24) immediately results in the nonanalytic contribution $\sim(1-z)^{\gamma-2}$ to $h(z)$. [One must also take into account that $h(1)=h_{1}(1)=1$ when a giant component is absent.] This corresponds to the power-law asymptotics of $\mathcal{P}(s)$. Remarkably, there is a qualitative difference in the component size distribution between undamaged networks and networks with randomly removed vertices or edges. In percolation problems for arbitrary uncorrelated networks, the power law for the distribution $\mathcal{P}(s)$ fails everywhere except a percolation threshold (see below).

In uncorrelated scale-free networks without a giant connected component, the largest connected component contains $\sim N^{1 /(\gamma-1)}$ vertices (Durrett, 2006; Janson, 2007), where we assume $\gamma>3$. As is natural, this size coincides with the cutoff $k_{\text {cut }}(N)$ in these networks.

Near the critical point in uncorrelated scale-free networks with a giant connected component, the size distribution of finite connected components to which a randomly chosen vertex belongs is

$$
\mathcal{P}(s) \sim s^{-\tau+1} e^{-s / s^{*}(p)},
$$

where $s^{*}\left(p_{c}\right) \rightarrow \infty$ : $\quad s^{*}(p) \sim\left(p-p_{c}\right)^{-1 / \sigma}$ near $p_{c}$ (Newman et al. , 2001). The distribution of the sizes of finite connected components is $\mathcal{P}_{s}(s) \sim \mathcal{P}(s) / s$. In uncorrelated networks with rapidly decreasing degree distributions, relation (25) is also valid in the absence of a giant connected component. Note that this situation, in particular, includes randomly damaged scale-free networkspercolation. The distribution $\mathcal{P}(s)$ near critical point in undamaged scale-free networks without a giant component, in simple terms, looks as follows: $\mathcal{P}(s) \sim s^{-\tau+1}$ at sufficiently small $s$, and $\mathcal{P}(s) \sim s^{-\gamma+1}$ at sufficiently large $s(\gamma>3$, in this region the inequality $\gamma>\tau$ is valid). Exponents $\tau, \sigma$, and $\beta$ satisfy the scaling relations $\tau-1=\sigma \beta+1=\sigma d_{u} / 2=d_{u} / d_{f}$. We stress that the mean size of a finite connected component, i.e., the first moment of the distribution $\mathcal{P}_{s}(s)$, is finite at the critical point. A divergent quantity (and an analogue of susceptibility) is the mean size of a finite connected component to which a randomly chosen vertex belongs,

$$
\langle s\rangle=\sum_{s} s \mathcal{P}(s) \sim\left|p-p_{c}\right|^{-\tilde{\gamma}}
$$

where $\tilde{\gamma}$ is the "susceptibility" critical exponent. This exponent does not depend on the form of the degree distribution. Indeed, the well-known scaling relation $\tilde{\gamma} / \nu=2-\eta$ with $\nu=1 / 2$ and $\eta=0$ substituted leads to $\tilde{\gamma}=1$ within the entire region $\gamma>3$.

The resulting exponents for finite connected components in the scale-free configuration model are as follows:

(i) for $\gamma>4$, the exponents are $\tau=5 / 2, \sigma=1 / 2, \tilde{\gamma}=$ 1 , which is also valid for classical random graphs; 
(ii) for $3<\gamma<4, \tau=2+1 /(\gamma-2), \sigma=(\gamma-3) /(\gamma-2)$, $\tilde{\gamma}=1$ (Cohen et al., 2003a).

The situation in the range $2<\gamma<3$ is not so clear. The difficulty is that in this interesting region, the giant connected component disappears at $p=0$, i.e., only with disappearance of the network itself. Consequently, one cannot separate "critical" and non-critical contributions, and so scaling relations fail. In this range,

(iii) i.e., for $2<\gamma<3,\langle s\rangle \propto p, \tau=3, \sigma=3-\gamma$.

Note that the last two values imply a specific cutoff of the degree distribution, namely $q_{\text {cut }} \sim N^{1 / 2}$.

In principle, the statistics of connected components in the bond percolation problem for a network may be obtained by analysing the solution of the $p$-state Potts model (Sec. VII) with $p=1$ placed on this net. Lee et al. (2004c) realized this approach for the static model.

The correlation volume of a vertex is defined as

$$
V_{i} \equiv \sum_{\ell=0} z_{\ell}(i) b^{\ell},
$$

where $z_{\ell}$ is the number of the $\ell$-th nearest neighbors of vertex $i$, and $b$ is a parameter characterizing the decay of correlations. The parameter $b$ may be calculated for specific cooperative models and depends on their control parameters, Sec. VI.C.4 In particular, if $b=1$, the correlation volume is reduced to the size of a connected component. Let us estimate the mean correlation volume in uncorrelated network with the mean branching coefficient $B=z_{2} / z_{1}: \bar{V} \sim \sum_{\ell}(b B)^{\ell}$ (we assume that the network has the giant connected component). So $\bar{V}(N \rightarrow \infty)$ diverges at and above the critical value of the parameter, $b_{c}=1 / B$. At the critical point, $\bar{V}\left(b_{c}\right)=\sum_{\ell} 1 \sim \ln N$. Since $B^{\bar{\ell}(N)} \sim N$, we obtain $\bar{V} \sim N^{\ln (b B) / \ln B}$ for $b>b_{c}$. Thus, as $b$ increases from $b_{c}$ to 1 , the exponent of the correlation volume grows from 0 to 1 .

The correlation volume takes into account remote neighbors with exponentially decreasing (if $b<1$ ) weights. A somewhat related quantity - the mean number vertices at a distance less than $a \bar{\ell}(N)$ from a vertex, where $a \geq 1$, - was analysed by López et al. (2007) in their study of "limited path percolation". This number is of the order of $N^{\delta}$, where exponent $\delta=\delta(a, B) \leq 1$.

\section{Finite size effects}

Practically all real-world networks are small, which makes the factor of finite size of paramount importance. For example, empirically studied metabolic networks contain about $10^{3}$ vertices. Even the largest artificial net - the World Wide Web, whose size will soon approach $10^{11}$ Web pages, show qualitatively strong finite size effects (Boguñá et al., 2004; Dorogovtsev and Mendes, 2002; May and Lloyd, 2001). To understand the strong effect of finite size in real scale-free networks one must recall that exponent $\gamma \leq 3$ in most of them, that is the second moment of a degree distribution diverges in the infinite network limit.

Note that the tree ansatz may be used even in this region $(\gamma \leq 3)$, where the uncorrelated networks are loopy. The same is true for at least the great majority of interacting systems on these networks. The reason for this surprising applicability is not clear up to now.

Let us demonstrate a poor-man's approach to percolation on a finite size (uncorrelated) network with $\gamma \leq 3$, where $p_{c}(N \rightarrow \infty) \rightarrow 0$. To be specific, let us, for example, find the size dependence of the percolation threshold, $p_{c}(N)$. The idea of this estimate is quite simple. Use Eq. (17), which was derived for an infinite network, but with the finite network's degree distribution substituted. Then, if the cutoff of the degree distribution is $q_{\text {cut }} \sim N^{1 / 2}$, we readily arrive at the following results:

$$
p_{c}(N, 2<\gamma<3) \sim N^{-(3-\gamma) / 2}, p_{c}(N, \gamma=3) \sim 1 / \ln N .
$$

These relations suggest the emergence of the noticeable percolation thresholds even in surprisingly large networks. In other words, the ultraresilience against random failures is effectively broken in finite networks.

Calculations of other quantities for percolation (and for a wide circle of cooperative models) on finite nets are analogous. Physicists, unlike mathematicians, routinely apply estimates of this sort to various problems defined on networks. Usually, these intuitive estimates work but evidently demand thorough verification. Unfortunately, a strict statistical mechanics theory of finite size effects for networks is technically hard and was developed only for very special models (see Sec. IV.A). For a phenomenological approach to this problem, see Sec. IX.B

\section{5. $k$-core architecture of networks}

The $k$-core of a network is its largest subgraph whose vertices have degree at least $k$ (Bollobás, 1984; Chalupa et al., 1979). In other words, each of vertices in the $k$-core has at least $k$ nearest neighbors within this subgraph. The notion of the $k$-core naturally generalizes the giant connected component and offers a more comprehensive view of the network organization. The $k$-core of a graph may be obtained by the "pruning algorithm" which looks as follows (see Fig. 8). Remove from the graph all vertices of degrees less than $k$. Some of remaining vertices may now have less than $k$ edges. Prune these vertices, and so on until no further pruning is possible. The result, if it exists, is the $k$-core. Thus, a network is hierarchically organized as a set of successfully enclosed $k$-cores, similarly to a Russian nesting doll"matrioshka". Alvarez-Hamelin et al. (2006) used this $k$-core architecture to produce a set of beautiful visualizations of diverse networks.

The $k$-core (bootstrap) percolation implies the breakdown of the giant $k$-core at a threshold concentration of vertices or edges removed at random from an infinite 


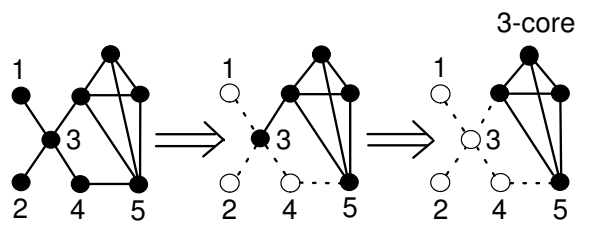

FIG. 8 Construction of the 3-core of a given graph. First we remove vertices 1,2 and 4 together with their links because they have degrees smaller than 3 . In the obtained graph, vertex 3 has degree 1 . Removing it, we get the 3 -core of the graph.

network. Pittel et al. (1996) found the way to analytically describe the $k$-core architecture of classical random graphs. More recently, Fernholz and Ramachandran (2004) mathematically proved that the $k$-core organization of the configuration model is asymptotically exactly described in the framework of a simple tree ansatz.

Let us discuss the $k$-core percolation in the configuration model with degree distribution $P(q)$ by using intuitive arguments based on the tree ansatz (Dorogovtsev et al., 2006a, b; Goltsev et al., 2006). The validity of the tree ansatz here is non-trivial since in this theory it is applied to a giant $k$-core which has loops. Note that in tree-like networks, $(k \geq 3)$-cores (if they exist) are giant - finite $(k \geq 3)$-cores are impossible. In contrast to the giant connected component problem, the tree ansatz in application to higher $k$-cores fails far from the $k$-core birth points. We assume that a vertex in the network is present with probability $p=1-Q$. In this locally tree-like network, the giant $k$-core coincides with the infinite $(k-1)$-ary subtree. By definition, the $m$-ary tree is a tree where all vertices have branching at least $m$.

Let the order parameter in the problem, $R$, be the probability that a given end of an edge of a network is not the root of an infinite $(k-1)$-ary subtree. (Of course, $R$ depends on $k$.) An edge is in the $k$-core if both ends of this edge are roots of infinite $(k-1)$-ary subtrees, which happens with the probability $(1-R)^{2}$. In other words,

$$
(1-R)^{2}=\frac{\text { number of edges in the } k \text {-core }}{\text { number of edges in the network }},
$$

which expresses the order parameter $R$ in terms of observables. Figure 9 graphically explains this and the following two relations. A vertex is in the $k$-core if at least $k$ of its neighbors are roots of infinite $(k-1)$-ary trees. So, the probability $M_{k}$ that a random vertex belongs to the $k$-core (the relative size of the $k$-core) is given by the equation:

$$
M_{k}=p \sum_{n \geq k} \sum_{q \geq n} P(q) C_{n}^{q} R^{q-n}(1-R)^{n},
$$

where $C_{n}^{q}=q ! /[(q-n) ! n !]$. To obtain the relative size of the $k$-core, one must substitute the physical solution of the equation for the order parameter into Eq. (30). We write the equation for the order parameter, noticing that (a)

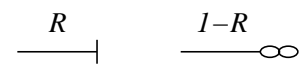

(b)

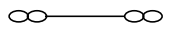

(c)

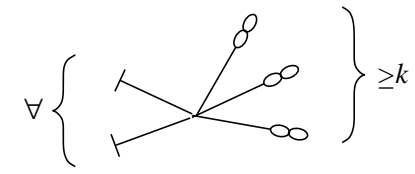

(d)

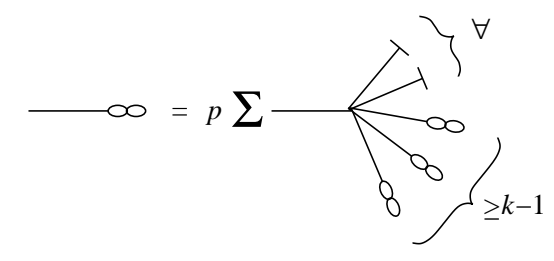

FIG. 9 Diagrammatic representation of Eqs. (29)-(31). (a) Graphic notations for the order parameter $R$ and for $1-R$. (b) The probability that both ends of an edge are in the $k$ core, Eq. (29). (c) Configurations contributing to $M_{k}$, which is the probability that a vertex is in the $k$-core, Eq. (30). The symbol $\forall$ here indicates that there may be any number of the nearest neighbors which are not trees of infinite $(k-1)$-ary subtrees. (d) A graphic representation of Eq. (31) for the order parameter. Adapted from Goltsev et al. (2006).

a given end of an edge is a root of an infinite $(k-1)$-ary subtree if it has at least $k-1$ children which are roots of infinite $(k-1)$-ary subtrees. Therefore,

$$
1-R=p \sum_{n=k-1}^{\infty} \sum_{i=n}^{\infty} \frac{(i+1) P(i+1)}{z_{1}} C_{n}^{i} R^{i-n}(1-R)^{n} .
$$

This equation strongly differs from that for the order parameter in the ordinary percolation, compare with Eq. (14). The solution of Eq. (32) at $k \geq 3$ indicates a quite unusual critical phenomenon. The order parameter (and also the size of the $k$-core) has a jump at the critical point like a first order phase transition. On the other hand, it has a square root critical singularity:

$$
R_{c}-R \propto\left[p-p_{c}(k)\right]^{1 / 2} \propto M_{k}-M_{k c},
$$

see Fig. 10. This intriguing critical phenomenon is often called a hybrid phase transition (Parisi and Rizzo, 2006; Schwartz et al., 2006). Relations (32) are valid if the second moment of the degree distribution is finite. Otherwise, the picture is very similar to what we observed for ordinary percolation. In this range, the $k$-cores, even of high order, practically cannot be destroyed by the random removal of vertices from an infinite network.

The 2-core of a graph can be obtained from the giant connected component of this graph by pruning dangling branches. At $k=2$, Eq. (31) for the order parameter is identical to Eq. (14) for the ordinary percolation. Therefore the birth point of the 2-core coincides with that of the giant connected component, and the phase transition 


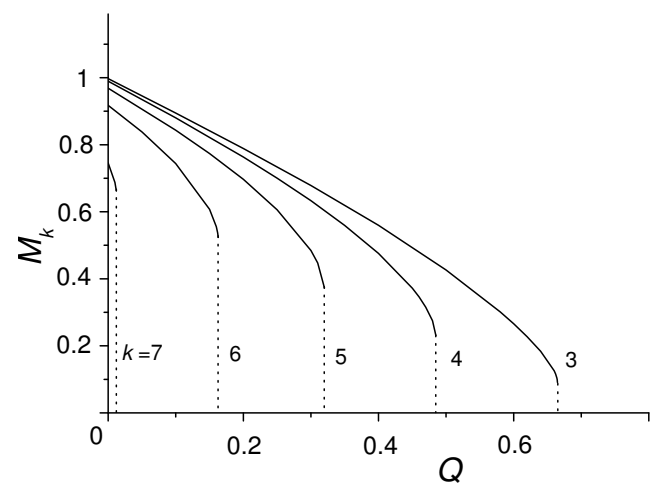

FIG. 10 Relative sizes of the $k$-cores, $M_{k}$, in classical random graphs with the mean degree $z_{1}=10$ versus the concentration $Q=1-p$ of randomly removed vertices.Adapted from Dorogovtsev et al. (2006a).

is continuous. According to Eq. (30) the size $M_{2}$ of the 2 -core is proportional to $(1-R)^{2}$ near the critical point, and so it is proportional to the square of the size of the giant connected component. This gives $M_{2} \propto\left(p-p_{c}\right)^{2}$ if the degree distribution decays rapidly.

In stark contrast to ordinary percolation, the birth of $(k>2)$-cores is not related to the divergence of corresponding finite components which are absent in tree-like networks. Then, is there any divergence associated with this hybrid transition? The answer is yes. To unravel the nature of this divergence, let us introduce a new notion. The $k$-core's corona is a subset of vertices in the $k$-core (with their edges) which have exactly $k$ nearest neighbors in the $k$-core, i.e., the minimum possible number of connections. One may see that the corona itself is a set of disconnected clusters. Let $N_{\text {crn }}$ be the mean total size of corona clusters attached to a vertex in the $k$-core. It turns out that it is $N_{\text {crn }}(p)$ which diverges at the birth point of the $k$-core,

$$
N_{\text {crn }}(p) \propto\left[p-p_{c}(k)\right]^{-1 / 2}
$$

(Goltsev et al., 2006; Schwartz et al., 2006). Moreover, the mean intervertex distance in the corona clusters diverges by the same law as $N_{\text {crn }}(p)$ (Goltsev et al., 2006). It looks like the corona clusters "merge together" exactly at the $k$-core percolation threshold and simultaneously disappear together with the $k$-core, which, of course, does not exist at $p>p_{c}(k)$.

Similarly to the mean size of a cluster to which a vertex belongs in ordinary percolation, $N_{\mathrm{crn}}$ plays the role of susceptibility in this problem, see Schwartz et al. (2006) for more detail. The exponent of the singularity in Eq. (33), 1/2, dramatically differs from the standard mean-field value of exponent $\tilde{\gamma}=1$ (see Sec. III.B.3). At this point, it is appropriate to mention a useful association. Recall the temperature dependence of the order parameter $m(T)$ in a first order phase transition. In normal thermodynamics, metastable states cannot be realized. Nonetheless, consider the metastable branch of

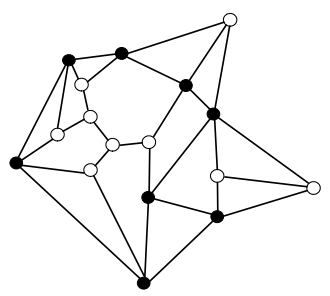

FIG. 11 3-core of a graph and its corona (removed vertices and links are not shown). The corona consists of a set of clusters with vertices (open circles) having exactly 3 nearest neighbors in this 3-core.

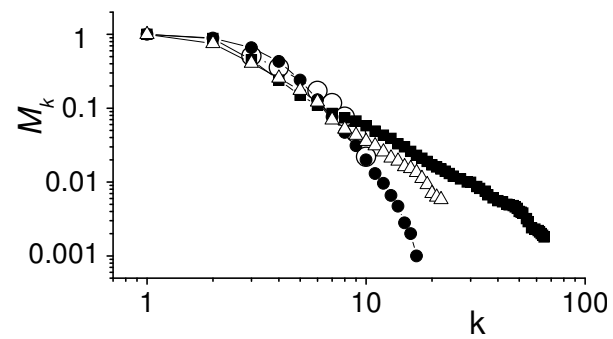

FIG. 12 Relative size of the $k$-cores vs. $k$ in several networks. $\bigcirc, M_{k}$ calculated neglecting correlations, by using the degree distribution of Internet router network, $N \approx 190000$, adapted from Dorogovtsev et al. (2006a). $\triangle$, measurements for the Autonomous System network (CAIDA map), $N=8542$, adapted from Alvarez-Hamelin et al. (2005b). •, results for a maximally random scale-free $(\gamma=2.5)$ network of $10^{6}$ vertices, and $\boldsymbol{\square}$, for a similar network but with a given strong clustering, $C=0.71$, adapted from Serrano and Boguná (2006a).

$m(T)$. One may easily find that near the end $\left(T_{0}\right)$ of this branch, $m(T)=m\left(T_{0}\right)+\operatorname{const}\left[T_{0}-T\right]^{1 / 2}$, and the susceptibility $\chi(T) \propto\left[T_{0}-T\right]^{-1 / 2}$. Compare these singularities with those of Eqs. (32) and (33). The only essential difference is that, in contrast to the $k$-core percolation, in the ordinary thermodynamics this region is not approachable. Parallels of this kind were discussed already by Aizenman and Lebowitz (1988).

By using Eqs. (30) and (31), we can easily find the $k$-core sizes, $M_{k}$ in the important range $2<\gamma<3$ :

$$
M_{k}=p^{1 /(3-\gamma)}\left(q_{0} / k\right)^{(\gamma-1) /(3-\gamma)},
$$

where $q_{0}$ is the minimal degree in the scale-free degree distribution (Dorogovtsev et al., 2006a). The exponent of this power law agrees with the observed one in a realworld network - the Internet at the Autonomous System level and the map of routers Alvarez-Hamelin et al., 2005b; Carmi et al., 2006b; Kirkpatrick, 2005). In the infinite scale-free networks of this kind, there is an infinite sequence of the $k$-cores (34). All these cores have a practically identical architecture - their degree distributions asymptotically coincide with the degree distribution of the network in the range of high degrees.

The finiteness of networks restricts the $k$-core sequence by some maximum number $k_{h}$ for the highest $k$-core. 
Goltsev et al. (2006) and Dorogovtsev et al. (2006a, b) estimated $k_{h}$ substituting empirical degree distributions into the equations for uncorrelated networks. Unfortunately, the resulting $k_{h}$ turned out to be several (3) times smaller than the observed values (Alvarez-Hamelin et al., 2005b; Carmi et al., 2007). Later Serrano and Boguñá (2006a, c) arrived at much more realistic $k_{h}$, taking into account high clustering (see Fig. 12). (They simulated a maximally random network with a given degree distribution and a given clustering.) There is also another way to diminish $k_{h}$ : random damaging first destroys the highest $k$-core, then the second highest, and so on.

\section{Percolation on degree-degree correlated networks}

Let in a random network only pair correlations between nearest neighbor degrees be present. Then this network has a locally tree-like structure, and so one can easily analyse the organization of connected components (Boguñá et al., 2003b; Newman, 2002b; Vázquez and Moreno, 2003). The network is completely described by the joint degree-degree distribution $P\left(q, q^{\prime}\right)$, see Sec. II.F (and, of course, by $N$ ). It is convenient to use a conditional probability $P\left(q^{\prime} \mid q\right)$ that if an end vertex of an edge has degree $q$, then the second end has degree $q^{\prime}$. In uncorrelated networks, $P\left(q^{\prime} \mid q\right)=q^{\prime} P\left(q^{\prime}\right) /\langle q\rangle$ is independent of $q$. Obviously, $P\left(q^{\prime} \mid q\right)=\langle q\rangle P\left(q, q^{\prime}\right) /[q P(q)]$. The important quantity in this problem is the probability $x_{q}$ that if an edge is attached to a vertex of degree $q$, then, following this edge to its second end, we will not appear in the giant connected component. For the sake of brevity, let us discuss only the site percolation problem, where $p$ is the probability that a vertex is retained. For this problem, equations for $x_{q}$ and an expression for the relative size of the giant connected component take the following form:

$$
\begin{aligned}
& x_{q}=1-p+p \sum_{q^{\prime}} P\left(q^{\prime} \mid q\right)\left(x_{q^{\prime}}\right)^{q^{\prime}-1}, \\
& 1-S=1-p+p \sum_{q} P(q)\left(x_{q}\right)^{q}
\end{aligned}
$$

(Vázquez and Moreno, 2003), which naturally generalizes Eqs. (14) and (15). Solving the system of equations (35) gives the full set $\left\{x_{q}\right\}$. Substituting $\left\{x_{q}\right\}$ into Eq. (36) provides $S$. Newman (2002b) originally derived these equations in a more formal way, using generating functions, and numerically solved them for various networks. The resulting curve $S(p)$ was found to significantly depend on the type of correlations - whether the degree-degree correlations were assortative or disassortative. Compared to an uncorrelated network with the same degree distribution, the assortative correlations increase the resilience of a network against random damage, while the disassortative correlations diminish this resilience. See Noh (2007) for a similar observation in another network model with correlations.
Equation (35) shows that the birth of the giant connected component is a continuous phase transition. The percolation threshold is found by linearizing Eq. (35) for small $y_{q}=1-x_{q}$, which results in the condition: $\sum_{q^{\prime}} C_{q q^{\prime}} y_{q^{\prime}}=0$, where the matrix elements $C_{q q^{\prime}}=$ $-\delta_{q q^{\prime}}+p\left(q^{\prime}-1\right) P\left(q^{\prime} \mid q\right)$. With this matrix, the generalization of the Molloy Reed criterion to the correlated networks is the following condition: if the largest eigenvalue of the matrix $C_{q q^{\prime}}$ is positive, then the correlated network has a giant connected component. The percolation threshold may be obtained by equating the largest eigenvalue of this matrix to zero. In uncorrelated networks this reduces to criterion (17).

Interestingly, the condition of ultra-resilience against random damage does not depend on correlations. As in uncorrelated networks, if the second moment $\left\langle q^{2}\right\rangle$ diverges in an infinite network, the giant connected component cannot be eliminated by random removal of vertices (Boguñá et al., 2003b; Vázquez and Moreno, 2003). Very simple calculations show that the mean number $z_{2}$ of the second nearest neighbors of a vertex in a degreedegree correlated network diverges simultaneously with $\left\langle q^{2}\right\rangle$. It is this divergence of $z_{2}$ that guarantees the ultraresilience.

Percolation and optimal shortest path problems were also studied for weighted networks with correlated weights (Wu et al., 2007a).

\section{The role of clustering}

The statistics of connected components in highly clustered networks, with numerous triangles (i.e., the clustering coefficient $C$ does not approach zero as $N \rightarrow \infty$ ), is a difficult and poorly studied problem. An important step to the resolution of this problem has been made by Serrano and Boguñá (2006a,b,c). These authors studied constructions of networks with given degree distributions and given mean clusterings of vertices of degree $q, C(q)$. It turns out that only if $C(q)<1 /(q-1)$, it is possible to build an uncorrelated network with a given pair of characteristics: $P(q)$ and $C(q)$. Since clustering of this kind does not induce degree-degree correlations, the regime $C(q)<1 /(q-1)$ was conventionally called "weak clustering". (When $C(q)<1 /(q-1)$, then the number of triangles based on an edge in the network is one or zero.) On the other hand, if $C(q)$ is higher than $1 /(q-1)$ at least at some degrees - "strong clustering",- - then the constructed networks necessarily have at least correlations between the degrees of the nearest neighbors.

Serrano and Boguñá (2006a,b, c) made a helpful simplifying assumption that the triangles in a network cannot have joint edges and neglected long loops. This assumption allowed them to effectively use a variation of the "tree ansatz". In particular they studied the bond percolation problem for these networks. The conclusions of this work are as follows:

(i) If the second moment of the degree distribution 


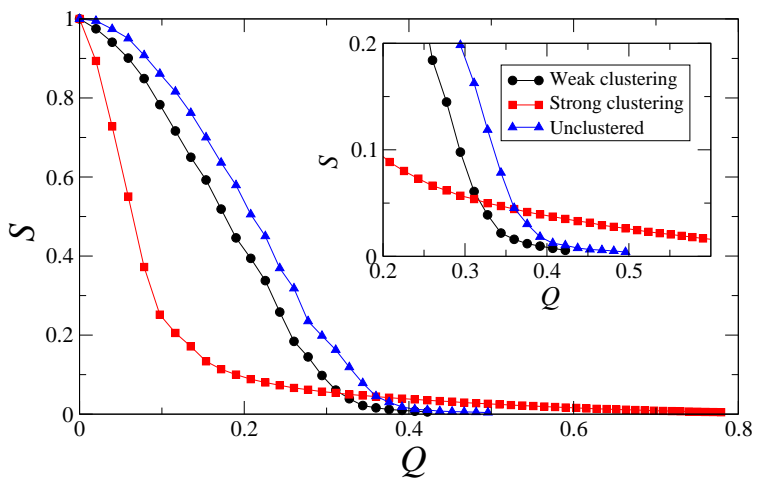

FIG. 13 Bond percolation on unclustered, and "strongly" and "weakly" clustered scale-free networks. Exponent $\gamma=$ 3.5. The relative size $S$ of the giant connected component is shown as a function of the concentration $Q=1-p$ of removed edges. From Serrano and Boguñá (2006c).

is finite, the "weak clustering" makes the network less resilient to random damage - the percolation threshold (in terms of $Q=1-p$, where $Q$ is the fraction of removed edges) decreases, see Fig. 13. Contrastingly, the "strong clustering" moves the percolation threshold in the opposite direction, although small damage (low $Q$ ) noticeably diminishes the giant connected component.

(ii) If the second moment of the degree distribution diverges, neither "weak" nor "strong" clustering can destroy the giant connected component in an infinite network.

Newman (2003b) proposed a different approach to highly clustered networks. He used the fact that a onemode projection of a bipartite uncorrelated network has high clustering, while the original bipartite network has a locally tree-like structure. (In this projection, two vertices of, say, type 1 , are the nearest neighbors if they have at least one joint vertex of type 2.) This convenient feature allows one to describe properties of the clustered one-partite network with a tunable clustering and a tunable degree distribution by applying the tree ansatz to the bipartite network. For details - applications to percolation and epidemic processes,- - see Newman (2003b).

\section{E. Giant component in directed networks}

The structure of the giant connected component in uncorrelated directed networks was studied by Dorogovtsev et al. (2001a). By definition, edges of directed networks are directed, so that the configuration model is described by the joint in-, out-degree distribution $P\left(q_{i}, q_{o}\right)$. Directed networks have a far more complex organization and topology of the giant connected components than undirected ones. This organization may include specifically interconnected giant subcomponents with different birth points. Applying the tree ansatz, these authors found the birth points of various giant components and obtained their sizes for an arbitrary $P\left(q_{i}, q_{o}\right)$, see also Schwartz et al. (2002). For more detailed description of the giant components in directed networks see Serrano and De Los Rios (2007).

Boguñá and Serrano (2005) generalized this theory to uncorrelated networks which contain both directed and undirected connections. These networks are characterized by a distribution $P\left(q, q_{i}, q_{o}\right)$, where $q, q_{i}$, and $q_{o}$ are the numbers of undirected, in-directed, and out-directed connections of a vertex, respectively.

The exponents of the critical singularities for the transitions of the birth of various giant connected components in directed networks were calculated by Schwartz et al. (2002). Note that although the in-, out-degrees of different vertices in these networks are uncorrelated, there may be arbitrary correlations between in- and out-degrees of the same vertex. The critical exponents, as well as the critical points, essentially dependent on these in-, outdegree correlations.

\section{F. Giant component in growing networks}

The intrinsic large-scale inhomogeneity of nonequilibrium (e.g., growing) networks may produce a surprising critical phenomenon. The large-scale inhomogeneity here means the difference between properties of vertices according to their age. This difference usually makes the "old" part of a growing network more "dense" than the "young" one.

Callaway et al. (2001) found an unexpected effect in the birth of the giant connected component already in a very simple model of the growing network. In their model, the network grows due to two parallel processes: (i) there is an inflow of new vertices with the unit rate, and, in addition, (ii) there is an inflow of edges with rate $b$, which interconnect randomly chosen vertex pairs. The rate $b$ plays the role of the control parameter. As one could expect, the resulting degree distribution is very simple exponential. The inspection of this network when it is already infinite shows that it has a giant connected component for $b>b_{c}$, where $b_{c}$ is some critical value, unimportant for us. Remarkably, the birth of the giant connected component in this net strongly resembles the famous Berezinskii-Kosterlitz-Thouless (BKT) phase transition in condensed matter (Berezinskii, 1970; Kosterlitz and Thouless, 1973). Near the critical point, the relative size of the giant connected component has the specific BKT singularity:

$$
S \propto \exp \left(- \text { const } / \sqrt{b-b_{c}}\right) .
$$

Note that in an equilibrium network with the same degree distribution, $S$ would be proportional to the small deviation $b-b_{c}$. The singularity (37), with all derivatives 
vanishing at the critical point, implies an infinite order phase transition.

Normally, the BKT transition occurs at the lower critical dimension of an interacting system, where critical fluctuations are strong, e.g., dimension 2 for the $X Y$ model. Most of known models with this transition have a continuous symmetry of the order parameter. So that the discovery of the BKT singularity in infinite dimensional small worlds, that is in the mean-field regime, was somewhat surprising. The mean size of a finite connected component to which a vertex belongs in this network was also found to be nontraditional. This characteristic - an analogy of susceptibility - has a finite jump at this transition and not a divergence generic for equilibrium networks and disordered lattices.

Dorogovtsev et al. (2001b) analytically studied a much wider class of growing networks with an arbitrary linear preferential attachment (which may be scale-free or exponential) and arrived at very similar results. In particular, they found that the constant and $b_{c}$ in Eq. (37) depend on the rules of the growth. Looking for clues and parallels with the canonical BKT transition, they calculated the size distribution of connected components, $\mathcal{P}_{s}(s)$, characterizing correlations. The resulting picture looks as follows.

- The distribution $\mathcal{P}_{s}(s)$ slowly (in a power-law fashion) decays in the whole phase without the giant connected component, and this distribution rapidly decreases in the phase with the giant connected component.

This picture is in stark contrast to the equilibrium networks, where

- the distribution $\mathcal{P}_{s}(s)$ slowly decays only at the birth point of the giant connected component (if a network is non-scale-free, see Sec. III.B.3.

In this respect, the observed transition in growing networks strongly resembles the canonical BKT transitions, where the critical point separates a phase with rapidly decreasing correlations and "a critical phase" with correlations decaying in a power-law fashion. (Note, however the inverted order of the phases with a power-law decay and with a rapid drop in these transitions.)

This phase transition was later observed in many other growing networks with exponential and scalefree degree distributions (only for some of these networks, see Lancaster (2002), Coulomb and Bauer (2003), Krapivsky and Derrida (2004), Bollobás and Riordan (2005), and Durrett (2006)). Moreover, even ordinary, "equilibrium" bond percolation considered on special networks has the same critical phenomenon. For example, (i) grow up an infinite random recursive graph (at each time step, add a new vertex and attach it to $m$ randomly chosen vertices of the graph), (ii) consider the bond percolation problem on this infinite network. We emphasize that the attachment must be only random here. It is easy to see that the resulting network may be equivalently prepared by using a stochastic growth process which just leads to the BKT-like transition. Similar effects were observed on the Ising and Potts models placed on growing networks (see Sec. VI.G.1). A more realistic model of a growing protein interaction network where a giant connected component is born with the BKT-type singularity was described by Kim et al. (2002).

Various percolation problems on deterministic (growing) graphs may be solved exactly. Surprisingly, percolation properties of deterministic graphs are rather similar to those of their random analogs. For detailed discussion of these problems, see, e.g., Dorogovtsev et al. (2002a), Dorogovtsev (2003), and Rozenfeld and ben-Avraham (2007).

\section{G. Percolation on small-world networks}

Let us consider a small-world network based on a $d$ dimensional hypercubic lattice $\left(N \cong L^{d}\right)$ with random shortcuts added with probability $\phi$ per lattice edge. Note that in this network, in the infinite network limit, there are no finite loops including shortcuts. All finite loops are only of lattice edges. This fact allows one to apply the usual tree ansatz to this actually loopy network. In this way Newman et al. (2002) obtained the statistics of connected components in the bond percolation problem for two-dimensional small-world networks. Their qualitative conclusions are also valid for bond and site percolation on one-dimensional (Moore and Newman, 2000a, b; Newman and Watts, 1999a, b) and arbitrary-dimensional small-world networks.

In the spirit of classical random graphs, at the percolation threshold point, $p_{c}$, there must be one end of a retained shortcut per connected component in the lattice substrate. In more strict terms, this condition is $2 d \phi p_{c}=1 /\left\langle n_{0}\right\rangle\left(p_{c}\right)$, i.e., the mean density of the ends of shortcuts on the lattice substrate must be equal to the mean size $\left\langle n_{0}\right\rangle$ of a connected component (on a lattice) to which a vertex belongs. In the standard percolation problem on a lattice, $\left\langle n_{0}\right\rangle(p) \propto\left(p_{c 0}-p\right)^{-\tilde{\gamma}}$, where $p_{c 0}$ and $\tilde{\gamma}$ are the percolation threshold and the "susceptibility" critical exponent in the standard percolation. So, the percolation threshold is displaced by

$$
p_{c 0}-p_{c} \propto \phi^{1 / \tilde{\gamma}}
$$

if $\phi$ is small (Warren et al., 2003). For example, for the bond percolation on the two-dimensional small-world network, $p_{c 0}=1 / 2$ and $\tilde{\gamma}=43 / 18=2.39 \ldots$. The mean size of a connected component to which a random vertex belongs is also easily calculated:

$$
\langle n\rangle=\left\langle n_{0}\right\rangle /\left(1-2 d \phi p\left\langle n_{0}\right\rangle\right) \propto\left(p_{c}-p\right)^{-1} .
$$

So that its critical exponent equals 1 , as in the classical random graphs. The other percolation exponents also 
coincide with their values for classical graphs. In general, this claim is equally valid for other cooperative models on small-world networks in a close environment of a critical point.

Ozana (2001) described the entire crossover from the lattice regime to the small-world one and finite size effects by using scaling functions with dimensionless combinations of the three characteristic lengths: (i) $L$, (ii) the mean Euclidean distance between the neighboring shortcut ends, $\xi_{\mathrm{sw}} \equiv 1 /(2 d \phi p)^{1 / d}$, and (iii) the usual correlation length $\xi_{1}$ for percolation on the lattice. For an arbitrary physical quantity, $X(L)=L^{x} f\left(\xi_{\mathrm{sw}} / L, \xi_{1} / L\right)$, where $x$ and $f($,$) are scaling exponent and function. In$ the case of $L \rightarrow \infty$, this gives $X=\xi_{\mathrm{sw}}^{y} \xi_{1}^{z} g\left(\xi_{\mathrm{sw}} / \xi_{1}\right)$, where $y, z$, and $g()$ are other scaling exponents and function. This scaling is equally applicable to many other cooperative models on small-world networks.

\section{H. $k$-clique percolation}

A possible generalization of percolation was put forward by Derénvi et al. (2005). They considered percolation on the complete set of the $k$-cliques of a network. The $k$-clique is a fully connected subgraph of $k$ vertices. Two $k$-cliques are adjacent if they share $k-1$ vertices. For example, the smallest non-trivial clique, the 3-clique, is a triangle, and so that two triangles must have a common edge to allow the "3-percolation".

In fact, Derénvi et al. (2005) described the birth of the giant connected component in the set of the $k$-cliques of a classical random graph - the Gilbert model. The $k$-clique graph has vertices - $k$-cliques - and edges - connections between adjacent $k$-cliques. The total number of $k$ cliques approximately equals $N^{k} p^{k(k-1) / 2} / k$ !. The degree distribution of this graph is Poissonian, and the mean degree is $\langle q\rangle \cong N k p^{k-1}$, which may be much less than the mean degree in the Gilbert model, $N p$.

Since the sparse classical random graphs have few $(k \geq 3)$-cliques, this kind of percolation obviously implies the dense networks with a divergent mean degree. The application of the Molloy-Reed criterion to the $k$-clique graph gives the birth point of the $k$-clique giant connected component

$$
p_{c}(k) N=\frac{1}{k-1} N^{(k-2) /(k-1)} \text { as } N \rightarrow \infty
$$

(for more detail, see Palla et al. (2007)).

The birth of the giant connected component in the $k$ clique graph looks quite standard and so that its relative size is proportional to the deviation $\left[p-p_{c}(k)\right]$ near the critical point. On the other hand, the relative size $S_{k}$ of the $(k \geq 3)$-clique giant connected component in the original graph (namely, the relative number of vertices in this component) evolves with $p$ in a quite different manner. This component emerges abruptly, and for any $p$ above the threshold $p_{c}(k)$ it contains almost all vertices of the network: $S_{k}\left(p<p_{c}(k)\right)=0$ and $S_{k}\left(p>p_{c}(k)\right)=1$.

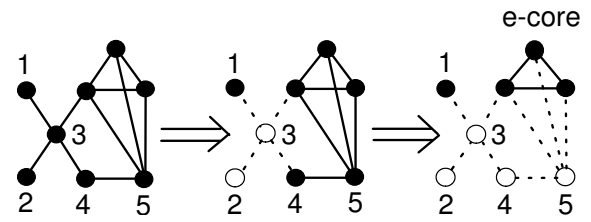

FIG. 14 Construction of the $e$-core of a given graph. Consequently removing the leaf [23] and a new leaf [45] we obtain one isolated vertex and the $e$-core of the graph. Removing at first the leaf [13] and then the leaf [45] leads to the same $e$-core and the same number of isolated vertices. Compare with the 3-core of the same graph in Fig. 8.

\section{I. $e$-core}

Let us define a leaf as the triple: a dead end vertex, its sole nearest neighbor vertex, and the edge between them. A more traditional definition does not include the neighbor, but here for the sake of convenience we modify it. A number of algorithms for networks are based on successive removal of these leaves from a graph. In particular, algorithms of this kind are used in the matching problem and in minimal vertex covers. Bauer and Golinelli (2001a) described the final result of the recursive removal of all leaves from the Erdős-Rényi graph. They found that if the mean degree $\langle q\rangle>e=2.718 \ldots$, the resulting network contains a giant connected component-we call it the e-core to distinct from resembling terms. The $e$-core is explained in Fig. 14, $e$-cores in other networks were not studied yet.

For $\langle q\rangle \leq e$, the removal procedure destroys the graph - only $O(N)$ isolated vertices and small connected components consisting in sum of $o(N)$ vertices remain. At $\langle q\rangle=e$, a second order phase transition of the birth of the $e$-core takes place. For $\langle q\rangle>e$, a finite fraction $S_{e}$ of $N$ vertices are in the $e$-core, a fraction $I$ are isolated vertices, and negligible fraction of vertices are in finite components. In the critical region,

$$
S_{e} \cong 12(\langle q\rangle-e) / e,
$$

and the mean degree $\langle q\rangle_{e}$ of the vertices in the $e$-core at the moment of its birth is exactly 2 which corresponds to a tree graph. In the critical region,

$$
\langle q\rangle_{e} \cong 2+\sqrt{8 / 3} \sqrt{\langle q\rangle-e} .
$$

This singularity is in sharp contrast to the analytic behavior of the mean degree of the usual giant connected component of this graph at the point of its birth. The relative number of isolated vertices has a jump only in the second derivative:

$$
I \cong \frac{3-e}{e}-\frac{1}{e}(\langle q\rangle-e)+\frac{1+3 \theta(\langle q\rangle-e)}{2 e}(\langle q\rangle-e)^{2},
$$

where $\theta(x<0)=0$ and $\theta(x>0)=1$. Interestingly, the leaf removal algorithm slows down as $\langle q\rangle$ approaches 
the critical point, which is a direct analog of the wellknown critical slowing down for usual continuous phase transitions.

The same threshold $\langle q\rangle=e$ is present in several combinatorial optimization problems on the classical random graphs. In simple terms, in each of these problems, a solution may be found "rapidly" only if $\langle q\rangle<e$. Above $e$, any algorithm applied needs a very long time. Note that this statement is valid both for the efficiently solvable in polynomial time, $\mathrm{P}$ (deterministic polynomialtime) problems and for the NP (non-deterministic polynomial time) problems. In particular, the $e$ threshold takes place in the matching $(P)$ problem - find in a graph the maximum set of edges without common vertices (Karp and Sipser, 1981), and, also, in the minimum vertex cover (NP) problem - if a guard sitting at a vertex controls the incident edges, find the minimum set of guards needed to watch over all the edges of a graph (Weigt and Hartmann, 2000). The matching problem, belonging to the $\mathrm{P}$ class (Aronson et al., 1998), is actually equivalent to the model of dimers with repulsion. We will discuss the minimum vertex cover in Sec.VI.E.3. Here we only mention that in the combinatorial optimization problems, the $e$ threshold separates the phase $\langle q\rangle<e$ with a "simple" structure of the "ground state", where the replica symmetry solution is stable from the phase with huge degeneracy of the "ground state", where the replica symmetry breaks. In particular, this degeneracy implies a huge number of minimum covers.

Note another class of problems, where leaf removal is essential. The adjacency matrix spectrum is relevant to the localization/delocalization of a quantum particle on a graph, see Sec. XII.E It turns out that that leaf removal does not change the degeneracy of the zero eigenvalue of the adjacency matrix, and so the $e$-core notion is closely related to the structure of this spectrum and to localization phenomena. Bauer and Golinelli (2001a) showed that the number of eigenvectors with zero eigenvalue equals the product $I N$, see Eq. (43), and thus has a jump in the second derivative at $\langle q\rangle=e$. See Sec. XII.E for more detail.

\section{CONDENSATION TRANSITION}

Numerous models of complex networks show the following phenomenon. A finite fraction of typical structural elements in a network (motifs) - edges, triangles, etc.- turn out to be aggregated into an ultra-compact subgraph with diameters much smaller than the diameter of this network. In this section we discuss various types of this condensation.

\section{A. Condensation of edges in equilibrium networks}

Networks with multiple connections. We start with rather simple equilibrium uncorrelated networks, where multiple connections, loops of length one, and other arbitrary configurations are allowed. There exist a number of more or less equivalent models of these networks (Bauer and Bernard, 2002; Berg and Lässig, 2002; Burda et al., 2001; Dorogovtsev et al., 2003b; Farkas et al., 2004). In many respects, these networks are equivalent to an equilibrium non-network systemballs statistically distributed among boxes - and so that they can be easily treated. On the other hand, the balls-in-boxes model has a condensation phase transition (Bialas et al., 1997; Burda et al., 2002).

We can arrive at uncorrelated networks with complex degree distribution in various ways. Here we mention two equivalent approaches to networks with a fixed number $N$ of vertices.

(i) Similarly to the balls-in-boxes model, one can define the statistical weights of the random ensemble members in the factorized form: $\prod_{i=1}^{N} p\left(q_{i}\right)$ (Burda et al., 2001), where the "one-vertex" probability $p(q)$ is the same for all vertices (or boxes) and depends on the degree of a vertex. If the number of edges $L$ is fixed, these weights additionally take into account the following constraint $\sum_{i} q_{i}=2 L$. With various $p(q)$ (and the mean degree $\langle q\rangle=2 L / N)$ we can obtain various complex degree distributions.

(ii) A more "physical", equivalent approach is as follows. A network is treated as an evolving statistical ensemble, where edges permanently change their positions between vertices (Dorogovtsev et al., 2003b). After relaxation, this ensemble approaches a final state - an equilibrium random network. If the rate of relinking factors into the product of simple, one-vertex-degree preference functions $f(q)$, the resulting network is uncorrelated. For example, one may choose a random edge and move it to vertices $i$ and $j$ selected with probability proportional to the product $f\left(q_{i}\right) f\left(q_{j}\right)$. The form of the preference function and $\langle q\rangle$ determines the distribution of connections in this network.

It turns out that in these equilibrium networks, scalefree degree distributions can be obtained only if $f(q)$ is a linear function. Furthermore, the value of the mean degree plays a crucial role. If, say, $f(q) \cong q+1-\gamma$ as $q \rightarrow \infty$, then three distinct regimes are possible. (i) When the mean degree is lower than some critical value $q_{c}$ (which is determined by the form of $f(q)$ ), the degree distribution $P(q)$ is an exponentially decreasing function. (ii) If $\langle q\rangle=q_{c}$, then $P(q) \sim q^{-\gamma}$ is scale-free. (iii) If $\langle q\rangle>q_{c}$, then one vertex attracts a finite fraction of all connections, in sum, $L_{\mathrm{ex}}=N\left(\langle q\rangle-q_{c}\right) / 2$ edges, but the other vertices are described by the same degree distribution as at the critical point. In other words, at $\langle q\rangle>q_{c}$, a finite fraction of edges are condensed on a single vertex, see Fig. 15(a). One can show that it is exactly one vertex that attracts these edges and not two or three or several. Notice a huge number of one-loops and multiple connections attached to this vertex. We emphasize that a scale-free degree distribution without condensation occurs only at one point - at the critical mean degree. This 
(a)

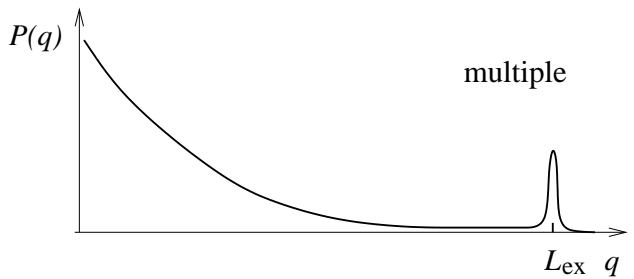

(b)

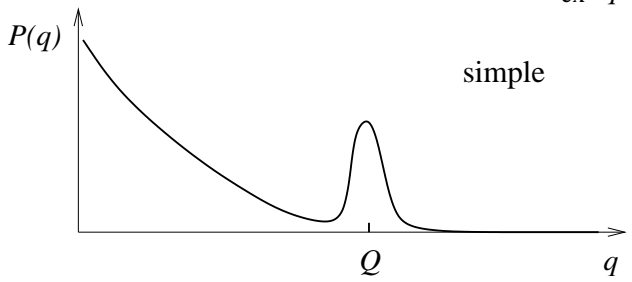

FIG. 15 Schematic plots of the degree distributions of the equilibrium networks with (a) and without (b) multiple connections in the condensation phase where the mean degree exceeds the critical value $q_{c}$ (Dorogovtsev et al., 2005). The peaks are due to a single vertex attracting $L_{\mathrm{ex}}=N\left(\langle q\rangle-q_{c}\right) / 2$ edges (a) or due to the highly interconnected core vertices of typical degree $Q \sim N / N_{h}(N)$ (b). Note the difference from the rich club phenomenon, where there are no such peaks in degree distributions. From Dorogovtsev et al. (2005).

is in contrast to networks growing under the mechanism of the preferential attachment, where linear preference functions generate scale-free architectures for wide range of mean degrees.

One can arrive at the condensation of edges in a quite different way. In the spirit of the work of Bianconi and Barabási (2001), who applied this idea to growing networks, let few vertices, or even a single vertex, be more attractive than others. Let, for example, the preference function for this vertex be $g f(q)$, where $f(q)$ is the preference function for the other vertices, and $g>1$ is a constant characterising a relative "strength" or "fitness" of this vertex. It turned out that as $g$ exceeds some critical value $g_{c}$, a condensation of edges on this "strong" vertex occurs (Dorogovtsev and Mendes, 2003). Interestingly, in general, this condensation is not accompanied by scale-free organization of the rest network.

Networks without multiple connections. If multiple connections and one-loops are forbidden, the structure of the condensate changes crucially. This difficult problem was analytically solved in Dorogovtsev et al. (2005). The essential difference from the previous case is only in the structure of the condensate. It turns out that in these networks, at $\langle q\rangle>q_{c}$, a finite fraction of edges, involved in the condensation, link together a relatively small, highly interconnected core of $N_{h}$ vertices, $N_{h}(N) \ll N$, Fig. 16. This core, however, is not fully interconnected, i.e., it is not a clique. (i) If the degree distribution $P(q)$ of this network decreases slower than any stretched exponential dependence, e.g., the network is scale-free, then $N_{h} \sim N^{1 / 2}$. (ii) In the case of a stretched exponential

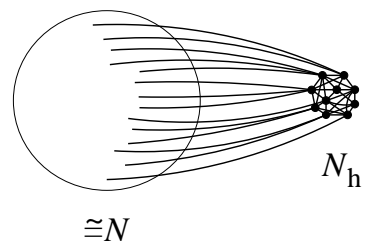

FIG. 16 The structure of a network without multiple connections when its vertex mean degree exceeds a critical value. The size $N_{h}(N)$ of the highly interconnected core varies in the range of $\sim N^{1 / 2}$ and $\sim N^{2 / 3}$ vertices. These vertices are interconnected by $\sim N$ edges. From Dorogovtsev et al. $(2005)$.

$P(q) \sim \exp \left(-\right.$ const $\left.q^{\alpha}\right), 0<\alpha<1$, the core consists of

$$
N_{h} \sim N^{(2-\alpha) /(3-\alpha)}
$$

vertices, that is the exponent of $N_{h}(N)$ is in the range $(1 / 2,2 / 3)$. The connections inside the core are distributed according to the Poisson law, and the mean degree $\sim N / N_{h}$ varies in the range from $\sim N / N^{1 / 2} \sim N^{1 / 2}$ to $\sim N / N^{2 / 3} \sim N^{1 / 3}$.

In the framework of traditional statistical mechanics, one can also construct networks with various correlations (Berg and Lässig, 2002), directed networks (Angel et al., 2006), and many others. Derénvi et al. (2004), Palla et al. (2004), and Farkas et al. (2004) constructed a variety of network ensembles, with statistical weights of members $\propto \prod_{i} \exp \left[-E\left(q_{i}\right)\right]$, where $E(q)$ is a given one-vertex degree function- "energy", as they called it. In particular, in the case $E(q)=-$ const $q \ln q$, these authors numerically found an additional, first-order phase transition. They studied a variation of the maximum vertex degree $q_{\max }$ in a network. As, say, $\langle q\rangle$ reaches $q_{c}$, a condensation transition takes place, and $q_{\text {max }}$ approaches the value $\sim N\langle q\rangle$, i.e., a finite fraction of all edges. Remarkably, at some essentially higher mean degree, $q_{c 2}, q_{\max }$ sharply, with hysteresis, drops to $\sim N^{1 / 2}$. That is, the network demonstrates a first order phase transition from the condensation ("star") phase to the "fully connected graph" regime.

\section{B. Condensation of triangles in equilibrium nets}

The condensation of triangles in network models was already observed in the pioneering work of Strauss (1986). Strauss proposed the exponential model, where statistical weights of graphs are

$$
W(g)=\exp \left[-\sum_{n} \beta_{n} E_{n}(g)\right] .
$$

Here $E_{n}(g)$ is a set of some quantities of a graph $g-$ a member of this statistical ensemble, and $\beta_{n}$ is a set of some positive constants. The reader may see that many modern studies of equilibrium networks are essentially 
based on the exponential model. Strauss included the quantity $E_{3}(g)$, that is the number of triangles in the graph $g$ taken with the minus sign, in the exponential. This term leads to the presence of a large number of triangles in the network. On the other, hand, they turn out to be very inhomogeneously distributed over the network. By simulating this (in his case, very small) network Strauss discovered that all triangles merge together forming a clique (fully connected subgraph) in the networkthe condensation of triangles.

Burda et al. (2004a, b) analytically described and explained this non-trivial phenomenon. Let us discuss the idea and results of their theory. The number of edges, $L$, and the number of triangles, $T$, in a network are expressed in terms of its adjacency matrix, $\hat{A}$. Namely, $L=\operatorname{Tr}\left(\hat{A}^{2}\right) / 2$ ! and $T=\operatorname{Tr}\left(\hat{A}^{3}\right) / 3$ !. The partition function of the Erdős-Rényi graph is simply $Z_{0}=\sum_{\hat{A}} \delta\left(\operatorname{Tr}\left(\hat{A}^{2}\right)-2 L\right)$, where sum is over all possible adjacency matrices. In the spirit of Strauss, the simplest generalization of the Erdős-Rényi ensemble, favoring triangles, has the following partition function

$$
Z=\sum_{\hat{A}} \delta\left(\operatorname{Tr}\left(\hat{A}^{2}\right)-2 L\right) e^{G T r\left(\hat{A}^{3}\right) / 3 !}=Z_{0}\left\langle e^{G T r\left(\hat{A}^{3}\right) / 3 !}\right\rangle_{0},
$$

where the constant $G$ quantifies the tendency to have many triangles, and $\langle\ldots\rangle_{0}$ denotes the averaging over the Erdős-Rényi ensemble. Equation (46) shows the form of the partition function for the canonical ensemble, i.e., with fixed $L$. In the grand canonical formulation, it looks more invariant: $Z_{g c}=$ $\sum_{\hat{A}} \exp \left[-C \operatorname{Tr}\left(\hat{A}^{2}\right) / 2 !+G \operatorname{Tr}\left(\hat{A}^{3}\right) / 3 !\right]$ (we here do not discuss the constant $C$ ). In fact, based on this form, Strauss (1986) argued that with $L / N$ finite and fixed, there exists a configuration, where all edges belong to a fully connected subgraph and so $\operatorname{Tr}\left(\hat{A}^{3}\right) \sim N^{3 / 2} \gg N$. Therefore, as $N \rightarrow \infty$, for any positive "interaction constant" $G$, the probability of realization of such a configuration should go to 1 , which is the stable state of this theory.

The situation, however, is more delicate. Burda et al. showed that apart from this stable condensation state, the network has a metastable, homogeneous one. These states are separated by a barrier, whose height approaches infinity as $N \rightarrow \infty$. So, in large networks (with sufficiently small $G$ ), it is practically impossible to approach the condensation state if we start evolutionrelaxation-from a homogeneous configuration. (Recall that Strauss numerically studied very small networks.)

Assuming small $G$, Burda et al. used the second equality in Eq. (46) to make a perturbative analysis of the problem. They showed that in the "perturbative phase", the mean number of triangles $\langle T\rangle=\left(\langle q\rangle^{3} / 6\right) \exp (G)$, where $\langle q\rangle$ is the mean degree of the network, see Fig. 17 In this regime, the number of triangles may be large, $\langle T\rangle \lesssim N$. Above the threshold $G_{t}(\langle q\rangle, N) \approx a \ln N+b$, where the coefficients $a$ and $b$ depend only on $\langle q\rangle$, the system easily jumps over the barrier and quickly approaches

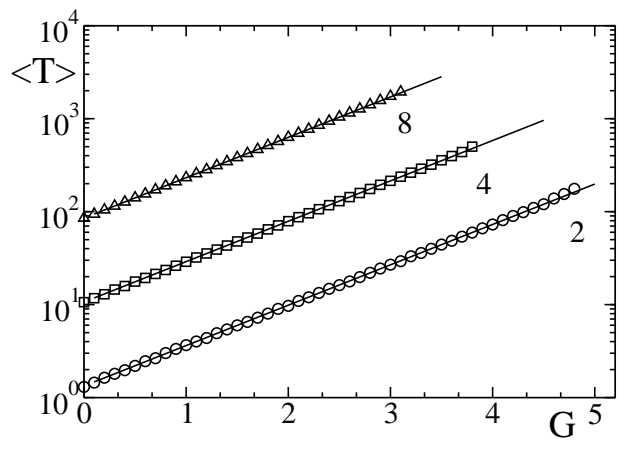

FIG. 17 The mean number of triangles $\langle T\rangle$ as a function of the parameter $G$ in the metastable state of the network of $N=2^{14}$ vertices for three values of the mean degree $\langle q\rangle=$ $2,4,8$. The dots are results of a simulation, and the lines are theoretical curves $\langle T\rangle=\left(\langle q\rangle^{3} / 6\right) \exp (G) \lesssim N$. The very right dot in each set corresponds to the threshold value $G_{t}(\langle q\rangle)$ above which the network quickly approaches the condensation state with $\langle T\rangle \sim N^{3 / 2}$. From Burda et al. (2004a).

the condensation state.

Burda et al. (2004b) generalized this theory to networks with complex degree distributions using the partition function $Z=\sum_{\hat{A}} \delta\left(\operatorname{Tr}\left(\hat{A}^{2}\right)-2 L\right) e^{G \operatorname{Tr}\left(\hat{A}^{3}\right)} \prod_{i}^{N} p\left(q_{i}\right)$, where $q_{i}$ is the degree of vertex $i$, and the weight $p(q)$ is given. In an even more general approach, $\operatorname{Tr}\left(\hat{A}^{3}\right)$ in the exponential should be replaced by a more general perturbation $S(\hat{A})$. Note that a different perturbation theory for the exponential model was developed by Park and Newman (2004a,b).

\section{Condensation of edges in growing networks}

Bianconi and Barabási (2001) discovered the condensation phase transition in networks, growing under the mechanism of preferential attachment. In their inhomogeneous network, preference function of vertices had a random factor ("fitness"): $g_{i} f\left(q_{i}\right)$ distributed according to a given function $p(g)$. Bianconi and Barabási indicated a class of sufficiently long-tailed distributions $p(g)$, for which an infinitely small fraction of vertices (maximally fitted ones) attract a finite fraction of edges. In fact, this condensation may be obtained even with a single more fitted vertex $(j): g_{i \neq j}=1, g_{j}=g>1$ (Dorogovtsev, and Mendes, 2001). In this case, the condensation on this vertex occurs in large networks of size $t \gg j$, if $g$ exceeds some critical value $g_{c}$.

Suppose that the network is a recursive graph, and the preference function $f(q)$ is linear. Then $g_{c}=\gamma_{0}-1$, where $\gamma_{0}$ is the exponent of the degree distribution of this network with all equal vertices $(g=1)$. Note that if the degree distribution is exponential $\left(\gamma_{0} \rightarrow \infty\right), g_{C} \rightarrow$ $\infty$, and the condensation is impossible. If $g<g_{c}$, the degree distribution of the network is the same as in the "pure" network. On the other hand, the phase with the 
condensate, $g>g_{c}$ has the following characteristics. (i) A finite fraction of edges $d \propto\left(g-g_{c}\right)$ is attached to the "fittest" vertex. (ii) The degree distribution exponent increases: $\gamma=1+g>\gamma_{0}$. (iii) In the entire condensation phase, relaxation to the final state (with the fraction $d$ of edges in the condensate) is very slow, of a power-law kind: $d_{j}(t)-d \sim t^{-\left(g-g_{c}\right) / g}$. Here $d_{j}(t)$ is a condensed fraction of edges at time $t$.

Bianconi and Barabási called this phenomenon the Bose-Einstein condensation based on evident parallels (in fact, this term was also applied sometimes to condensation in equilibrium networks, in the balls-in-boxes model, and in zero-range processes). We emphasize the completely classical nature of this condensation.

Kim et al. (2005) and Minnhagen et al. (2004) introduced a wide class of equilibrium and growing networks, where complex architectures are results of the process of merging and splitting of vertices. In many of these networks (where vertices differ from each other only by their degrees) the condensation of edges takes place. This phenomenon in the networks with aggregation was studied by Alava and Dorogovtsev (2005).

\section{CRITICAL EFFECTS IN THE DISEASE SPREADING}

The epidemic spreading in various complex networks was quite extensively studied in recent years, and it is impossible here to review in detail and even cite numerous works on this issue. In this section we only explain basic facts on the spread of diseases in networks, discuss relations to other phenomena in complex networks, and describe several recent results. The reader may refer to Pastor-Satorras and Vespignani (2003, 2004) for a comprehensive introduction to this topic.

\section{A. The SIS, SIR, SI, and SIRS models}

Four basic models of epidemics are widely used: the SIS, SIR, SI, and SIRS models, see, e.g., Nâsell (2002). $\mathrm{S}$ is for susceptible, $\mathrm{I}$ is for infective, and $\mathrm{R}$ is for recovered (or removed). In the network context, vertices are individuals, which are in one of these three $(S, I, R)$ or two $(\mathrm{S}, \mathrm{I})$ states, and infections spread from vertex to vertex through edges. Note that an ill vertex can infect only its nearest neighbors: $\mathrm{S} \rightarrow \mathrm{I}$.

The SIS model describes infections without immunity, where recovered individuals are susceptible. In the SIR model, recovered individuals are immune forever, and do not infect. In the SI model, recovery is absent. In the SIRS model, the immunity is temporary. The SIS, SIR, and SI models are particular cases of the more general SIRS model. We will touch upon only first three models.

Here we consider a heuristic approach of Pastor-Satorras and Vespignani (2001, 2003). This (a kind of mean-field) theory fairly well describes the epidemic spreading in complex networks. For a more strict approach, see, e.g., Newman (2002a), Kenah and Robins (2006), and references therein.

Let a network have only degree-degree correlations, and so it is defined by the conditional probability $P\left(q^{\prime} \mid q\right)$, see Sec. III.C. Consider the evolution of the probabilities $i_{q}(t), s_{q}(t)$, and $r_{q}(t)$ that a vertex of degree $q$ is in the I, S, and R states, respectively. For example, $i_{q}(t)=$ (number of infected vertices of degree $q$ ) $/[N P(q)]$. As is natural, $i_{q}(t)+s_{q}(t)+r_{q}(t)=1$. Let $\lambda$ be the infection rate. In other words, a susceptible vertex becomes infected with the probability $\lambda$ (per unit time) if at least one of the nearest neighbors is infected. Remarkably, $\lambda$ is actually the sole parameter in the SIS and SIR modelsother parameters can be easily set to 1 by rescaling. Here we list evolution equations for the SIS, SIR, and SI models. For derivations, see Boguñá et al. (2003b). However, the structure of these equations is so clear that the reader can easily explain them himself or herself, exploiting obvious similarities with percolation.

The SIS model. In this model, infected vertices become susceptible with unit rate, $r_{q}(t)=0, s_{q}(t)=1-i_{q}(t)$. The equation is

$$
\frac{d i_{q}(t)}{d t}=-i_{q}(t)+\lambda q\left[1-i_{q}(t)\right] \sum_{q^{\prime}} P\left(q^{\prime} \mid q\right) i_{q^{\prime}}(t) .
$$

The SIR model. In this model, infected vertices become recovered with unit rate. Two equations describe this system:

$$
\begin{aligned}
& \frac{d r_{q}(t)}{d t}=i_{q}(t) \\
& \frac{d i_{q}(t)}{d t}=-i_{q}(t)+\lambda q\left[1-i_{q}(t)\right] \sum_{q^{\prime}} \frac{q^{\prime}-1}{q^{\prime}} P\left(q^{\prime} \mid q\right) i_{q^{\prime}}(t) .
\end{aligned}
$$

Note the factor $\left(q^{\prime}-1\right) / q^{\prime}$ in the sum. This ratio is due to the fact that an infected vertex in this model cannot infect back its infector, and so one of the $q^{\prime}$ edges is effectively blocked.

The SI model. Here infected vertices are infected forever, $s_{q}(t)=1-i_{q}(t)$, and the dynamics is described by the following equation:

$$
\frac{d i_{q}(t)}{d t}=\lambda q\left[1-i_{q}(t)\right] \sum_{q^{\prime}} \frac{q^{\prime}-1}{q^{\prime}} P\left(q^{\prime} \mid q\right) i_{q^{\prime}}(t)
$$

(compare with Eq. (48)). This simplest model has no epidemic threshold. Moreover, in this model, $\lambda$ may be set to 1 without loss of generality.

If a network is uncorrelated, simply substitute $P\left(q^{\prime} \mid q\right)=q^{\prime} P\left(q^{\prime}\right) /\langle q\rangle$ into these equations. It is convenient to introduce $\Theta=\sum_{q^{\prime}}\left(q^{\prime}-1\right) P\left(q^{\prime}\right) i_{q^{\prime}}\langle q\rangle$ (for the SIR model) or $\Theta=\sum_{q^{\prime}} q^{\prime} P\left(q^{\prime}\right) i_{q^{\prime}}\langle q\rangle$ (for the SIS model) and then solve a simple equation for this degree-independent quantity. We stress that the majority of results on epidemics in complex networks were obtained by using only Eqs. (47), (48), and (49). Note that one can also analyse 
these models assuming a degree-dependent infection rate $\lambda$ (Giuraniuc et al., 2006).

\section{B. Epidemic thresholds and prevalence}

The epidemic threshold $\lambda_{c}$ is a basic notion in epidemiology. Let us define the fractions of infected and recovered (or removed) vertices in the final state as $i(\infty)=$ $\sum_{q} P(q) i_{q}(t \rightarrow \infty)$ and $r(\infty)=\sum_{q} P(q) r_{q}(t \rightarrow \infty)$, respectively. Below the epidemic threshold, $i(\infty)=r(\infty)=0$. In epidemiology the fraction $i(t)$ of infected vertices in a network is called the prevalence. The On the other hand, above the epidemic thresholds, (i) in the SIS model, $i\left(\infty, \lambda>\lambda_{c}^{S I S}\right)$ is finite, and (ii) in the SIR model, $i\left(\infty, \lambda>\lambda_{c}^{S I R}\right)=0$ and $r\left(\infty, \lambda>\lambda_{c}^{S I R}\right)$ is finite.

The linearization of Eqs. (47), (48), and (49) readily provide the epidemic thresholds. The simplest SI model on any network has no epidemic threshold - all vertices are infected in the final state, $i_{q}(t \rightarrow \infty)=1$. Here we only discuss results for uncorrelated networks (Pastor-Satorras and Vespignani, 2001, 2003), for correlated networks, see Boguná et al. (2003b). The reader can easily check that the SIS and SIR models have the following epidemic thresholds:

$$
\lambda_{c}^{S I S}=\frac{\langle q\rangle}{\left\langle q^{2}\right\rangle}, \quad \lambda_{c}^{S I R}=\frac{\langle q\rangle}{\left\langle q^{2}\right\rangle-\langle q\rangle} .
$$

Notice the coincidence of $\lambda_{c}^{S I R}$ with the percolation threshold $p_{c}$ in these networks, Eq. (17). (Recall that for bond and site percolation problems, $p_{c}$ is the same.) This coincidence is not occasional - strictly speaking, the SIR model is equivalent to dynamic percolation (Grassberger, 1983). In more simple terms, the SIR model, in the respect of its final state, is practically equivalent to the bond percolation problem [see Hastings (2006) for discussion of some difference, see also discussions in Kenah and Robins (2006) and Miller (2007)]. Equation (50) shows that general conclusions for percolation on complex networks are also valid for the SIS and SIR models. In particular, (i) the estimates and conclusions for $p_{c}$ from Secs. III.B.2, III.B.3, and III.B.4 are valid for the SIS and SIR models (simply replace $p_{c}$ by $\lambda_{c}^{S I S}$ or $\lambda_{c}^{S I R}$ ), the finite size relations also work; and (ii) the estimates and conclusions for the size $S$ of the giant connected component from these sections are also valid for $i(\infty)$ in the SIS model and for $r(\infty)$ in the SIR model, i.e., for prevalence.

In particular, Pastor-Satorras and Vespignani (2001) discovered that in uncorrelated networks with diverging $\left\langle q^{2}\right\rangle$, the epidemic thresholds approach zero value, but a finite epidemic threshold is restored if a network is finite (Boguñá et al., 2004; Mav and Lloyd, 2001; Pastor-Satorras and Vespignani, 2002a). Similarly to percolation, the same condition is valid for networks with degree-degree correlations (Boguñá et al., 2003a; Moreno and Vázquez, 2003).
The statistics of outbreaks near an epidemic threshold in the SIR model is similar to that for finite connected components near the birth point of a giant component. In particular, at a (SIR) epidemic threshold in a network with a rapidly decreasing degree distribution, the maximum outbreak scales as $N^{2 / 3}$ and the mean outbreak scales as $N^{1 / 3}$ (Ben-Naim and Krapivsky, 2004). (In the SIS model, the corresponding quantities behave as $N$ and $N^{1 / 2}$.) These authors also estimated duration of epidemic outbreaks. At a SIR epidemic threshold in these networks, the maximum duration of an outbreak scales as $N^{1 / 3}$, the average duration scales as $\ln N$, and the typical duration is of the order of one.

Interestingly, some of results on the disease spreading on complex networks were obtained before those for percolation, see the work of Pastor-Satorras and Vespignani (2001). For example, they found that in the SIS and SIR model on the uncorrelated scale-free network with degree distribution exponent $\gamma=3$, the final prevalence is proportional to $\exp [-g(\langle q\rangle) / \lambda)]$. Here $g(\langle q\rangle)$ depends only on the mean degree. That is, all derivatives of the prevalence over $\lambda$ equal zero at this specific point (recall the corresponding result for percolation). Furthermore, Boguñá and Pastor-Satorras (2002) fulfilled numerical simulations of the SIS model on the growing network of Callaway et al. (2001) and observed prevalence proportional to $\exp \left[-\right.$ const $\left./ \sqrt{\lambda-\lambda_{c}}\right]$, i.e., the Berezinskii-Kosterlitz-Thouless singularity.

Disease spreading was also studied in many other networks. For example, for small-world networks, see Moore and Newman (2000a); Newman (2002a); Newman et al. (2002) and references therein. For epidemics in networks with high clustering, see Newman (2003b); Petermann and De Los Rios (2004); Serrano and Boguñá (2006a). A very popular topic is various immunization strategies, see Cohen et al. (2003b); Dezsõ and Barabási (2002); Gallos et al. (2007a); Pastor-Satorras and Vespignani (2002b, 2003), and many other works.

Note that the excitation of a system of coupled neurons in response to external stimulus, in principle, may be considered similarly to the disease spreading. Excitable networks with complex architectures were studied in Kinouchi and Copelli (2006), Copelli and Campos (2007), and Wu et al. (2007b).

\section{Evolution of epidemics}

Equations (47), (48), and (49) describe the dynamics of epidemics. Let us discuss this dynamics above an epidemic threshold, where epidemic outbreaks are giant, that is involve a finite fraction of vertices in a network (Barthelemy et al., 2004, 2005; Moreno et al., 2002; Vazquez, 2006a). The demonstrative SI model is especially easy to analyse. A characteristic time scale of the epidemic outbreak can be trivially obtained in the following way (Barthelemy et al., 2004, 2005). Let the initial 


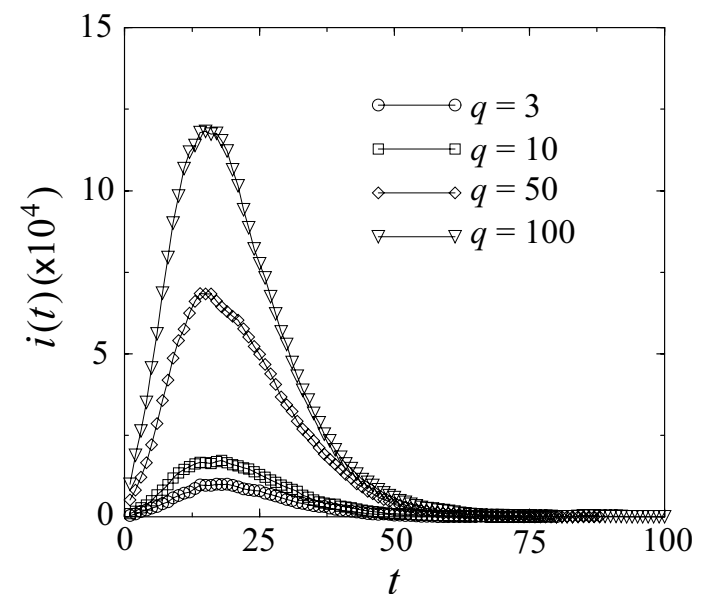

FIG. 18 The evolution of the average fraction of infected vertices in the SIR model on the Barabási-Albert network of $10^{6}$ vertices for various initial conditions. At $t=0$, randomly chosen vertices of a given degree $q$ are infected. The spreading rate is $\lambda=0.09$ which is above the epidemic threshold of this finite network. From Moreno et al. (2002).

condition be uniform, $i_{q}(t=0)=i_{0}$. Then in the range of short times the prevalence $i(t)=\sum_{q} P(q) i_{q}(t)$ rises according to the law:

$$
\frac{i(t)-i_{0}}{i_{0}}=\frac{\langle q\rangle^{2}-\langle q\rangle}{\left\langle q^{2}\right\rangle-\langle q\rangle}\left(e^{t / \tau}-1\right)
$$

with the time scale

$$
\tau=\frac{\langle q\rangle}{\lambda\left(\left\langle q^{2}\right\rangle-\langle q\rangle\right)} .
$$

Thus $\tau$ decreases with increasing $\left\langle q^{2}\right\rangle$. As is natural, the law (51) is violated at long times, when $i(t) \sim 1$. Expressions for $\tau$ in the SIS and SIR models are qualitatively similar to Eq. (52).

Notice some difference between the SIR and SIS (or SI) models. In the SIS and SI models, the fraction of infected vertices $i(t)$ monotonously grows with time until it approaches the final stationary state. Adversely, in the SIR model, $i(t)$ shows a peak - outbreak - at $t \sim \tau$ and approaches zero value as $t \rightarrow \infty$. As a result of heterogeneity of a complex network, the epidemic outbreaks strongly depend on initial conditions, actually on a first infected individual. Figure 18 shows how the average fraction of infected vertices evolves in the SIR model placed on the Barabási-Albert network if the first infected individual has exactly $q$ neighbors (Moreno et al., 2002). The spreading rate is supposed to be above the epidemic threshold. If this $q$ is large, then the outbreak is giant with high probability. On the other hand, if $q$ is small, then, as a rule, the infection disappears after a small outbreak, and the probability of a giant outbreak is low.

When $\left\langle q^{2}\right\rangle$ diverges $(\gamma \leq 3)$, Eqs. (51) and (52) are not applicable. Vazquez (2006a) considered disease spreading in this situation on a scale-free growing (or causal) tree.
Actually he studied a variation of the SI model, with an "average generation time" $T_{G} \sim 1 / \lambda$. In this model he analytically found

$$
d i(t) / d t \propto t^{\ell_{\max }-1} e^{-t / T_{G}},
$$

where $\ell_{\max }(N)$ is the diameter of the network (the maximum intervertex distance). Vazquez compared this dependence with his numerical simulations of the SI model on a generated network and a real-world one (the Internet at the Autonomous System level). He concluded that Eq. (53) provides a reasonable fitting to these results even in rather small networks.

\section{THE ISING MODEL ON NETWORKS}

The Ising model, named after the physicist Ernst Ising, is an extremely simplified mathematical model describing the spontaneous emergence of order. Despite its simplicity, this model is valuable for verification of general theories and assumptions, such as scaling and universality hypotheses in the theory of critical phenomena. What is important is that many real systems can be approximated by the Ising model. The Hamiltonian of the model is

$$
\mathcal{H}=-\sum_{i<j} J_{i j} a_{i j} S_{i} S_{j}-\sum_{i} H_{i} S_{i},
$$

where the indices $i$ and $j$ numerate vertices on a network, $i, j=1,2 \ldots N . a_{i j}$ is an element of the adjacency matrix: $a_{i j}=1$ or 0 if vertices $i$ and $j$ are connected or disconnected, respectively. Network topology is encoded in the adjacency matrix. In general, couplings $J_{i j}$ and local fields $H_{i}$ can be random parameters.

What kind of a critical behavior one might expect if we put the Ising model on the top of a complex network? Is it the standard mean-field like behavior? A naive answer is yes because a complex network is an infinitedimensional system. Indeed, it is generally accepted that the critical behavior of the ferromagnetic Ising model on a $d$-dimensional lattice at $d>4$ is described by the simple mean-field theory which assumes that an average effective magnetic field $H+J z_{1} M$ acts on spins, where $M$ is an average magnetic moment and $z_{1}=\langle q\rangle$ is the mean number of the nearest neighbors. An equation

$$
M=\tanh \left[\beta H+\beta J z_{1} M\right]
$$

determines $M$. This theory predicts a second order ferromagnetic phase transition at the critical temperature $T_{\mathrm{MF}}=J z_{1}$ in zero field with the standard critical behavior: $M \sim \tau^{\beta}, \chi=d M / d H \sim|\tau|^{-\tilde{\gamma}}$, where $\tau \equiv T_{\mathrm{MF}}-T, \beta=1 / 2$, and $\tilde{\gamma}=1$. First investigations of the ferromagnetic Ising model on the WattsStrogatz networks revealed the second order phase transition (Barrat and Weigt, 2000; Gitterman, 2000; Herrero, 2002; Hong et al., 2002b). This result qualitatively agreed with the simple mean-field theory. 
Numerical simulations of the ferromagnetic Ising model on a growing Barabási-Albert scale-free network (Aleksiejuk et al., 2002) demonstrated that the critical temperature $T_{c}$ increases logarithmically with increasing $N: T_{c}(N) \sim \ln N$. Therefore, in the thermodynamic limit, the system is ordered at any finite $T$. The simple mean-field theory fails to explain this behavior. Analytical investigations (Dorogovtsev et al., 2002b; Leone et al., 2002) based on a microscopic theory revealed that the critical behavior of the ferromagnetic Ising model on complex networks is richer and extremely far from that expected from the standard mean-field theory. They showed that the simple mean-field theory does not take into account the strong heterogeneity of networks.

In the present section, we look first at exact and approximate analytical methods (see also Appendices A, B. C) and then consider critical properties of ferro- and antiferromagnetic, spin-glass and random-field Ising models on complex networks.

\section{A. Main methods for tree-like networks}

\section{Bethe approach}

The Bethe-Peierls approximation is one of the most powerful methods for studying cooperative phenomena (Domb, 1960). It was proposed by Bethe (1935) and then applied by Peierls (1936) to the Ising model. This approximation gives a basis for developing a remarkably accurate mean-field theory. What is important, it can be successfully used to study a finite system with a given quenched disorder.

The list of modern applications of the Bethe-Peierls approximation ranges from solid state physics, information and computer sciences (Pearl, 1988), for example, image restoration (Tanaka, 2002), artificial vision (Freeman et al., 2000), decoding of error-correcting codes (McEliece et al., 1998), combinatorial optimization problems (Mézard and R. Zecchina, 2002), medical diagnosis (Kappen, 2002) to social models.

Let us consider the Ising model Eq. (54) on an arbitrary complex network. In order to calculate magnetic moment of a spin $S_{i}$, we must know the total magnetic field $H_{i}^{(t)}$ which acts on this spin. This gives $M_{i}=\left\langle S_{i}\right\rangle=\tanh \left[\beta H_{i}^{(t)}\right]$, where $\beta=1 / T . H_{i}^{(t)}$ includes both a local field $H_{i}$ and fields created by nearest neighboring spins. The spins interact with their neighbors who in turn interact with their neighbors, and so on. As a result, in order to calculate $H_{i}^{(t)}$ we have to account for all spins in the system. It is a hard work.

Bethe and Peierls proposed to take into account only interactions of a spin with its nearest neighbors. Interactions of these neighbors with remaining spins on a network were included in "mean fields". This simple idea reduces the problem of $N$ interacting spins to a problem of a finite cluster.

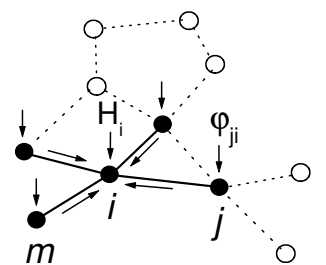

FIG. 19 A cluster on a graph. Within the Bethe-Peierls approach we choose a cluster consisting of spin $i$ and its nearest neighbors (closed circles). Cavity fields $\varphi_{j \backslash i}$ (vertical arrows) take into account interactions with remaining spins (dotted lines and open circles). $H_{i}$ is a local field. Arrows along edges show fields created by neighboring spins at vertex $i$.

Consider a cluster consisting of a central spin $S_{i}$ and its nearest neighbors $S_{j}$, see Fig. 19] The energy of the cluster is

$$
H_{\mathrm{cl}}=-\sum_{j \in N(i)} J_{i j} S_{i} S_{j}-H_{i} S_{i}-\sum_{j \in N(i)} \varphi_{j \backslash i} S_{j},
$$

where $N(i)$ means all vertices neighboring vertex $i$. Interactions between the spins $j \in N(i)$ are neglected. They will be approximately taken into account by the fields $\varphi_{j \backslash i}$. These fields are called cavity fields within the cavity method (Mézard and Parisi, 2001). The cavity fields must be found in a self-consistent way.

It is easy to calculate the magnetic moments of spins in the cluster. The magnetic field $H_{i}^{(t)}$ acting on $i$ is

$$
H_{i}^{(t)}=H_{i}+\sum_{j \in N(i)} h_{j i}
$$

where $h_{j i}$ is an additional field created by a spin $S_{j}$ at vertex $i$ (see Fig. 19):

$$
\tanh \beta h_{j i} \equiv \tanh \beta J_{i j} \tanh \beta \varphi_{j \backslash i} .
$$

In turn the field $H_{j}^{(t)}$ acting on spin $j$ is

$$
H_{j}^{(t)}=\varphi_{j \backslash i}+h_{i j},
$$

where the additional field $h_{i j}$ is created by the central spin $i$ at vertex $j$. This field is related to the additional fields in Eq. (58) as follows:

$$
\tanh \beta h_{i j}=\tanh \beta J_{i j} \tanh \left[\beta\left(H_{i}+\sum_{m \in N(i) \backslash j} h_{m i}\right)\right],
$$

where $N(i) \backslash j$ means all vertices neighboring vertex $i$, except $j$. In the framework of the belief-propagation algorithm (Sec. VI.A.2) the additional fields $h_{j i}$ are called messages. Using this vivid term, we can interpret Eq. (60) as follows (see Fig. 20). An outgoing message sent by spin $i$ to neighbor $j$ is determined by incoming messages which spin $i$ receives from other neighbors $m \in N(i)$, except $j$. Note that if vertex $i$ is a dead end, 


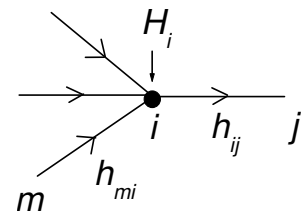

FIG. 20 Diagram representation of Eq. (60). An outgoing message $h_{i j}$ from vertex $i$ to vertex $j$ is determined by the local field $H_{i}$ and incoming messages to $i$ excluding the message from $j$.

then from Eq. (60) we obtain that the message $h_{i j}$ from $i$ to the only neighbor $j$ is determined by a local field $H_{i}$ :

$$
\tanh \beta h_{i j}=\tanh \beta J_{i j} \tanh \left(\beta H_{i}\right) .
$$

We can choose a cluster in which $S_{j}$ is the central spin. The field $H_{j}^{(t)}$ is given by the same Eq. (57). Comparing Eq. (57), where $j$ replaces $i$, with Eq. (59), we obtain

$$
\varphi_{i \backslash j}=H_{i}+\sum_{m \in N(i) \backslash j} h_{m i} .
$$

Equations (57)- 62) establish relations between the fields $\left\{h_{i j}\right\}$ and $\left\{\varphi_{i \backslash j}\right\}$. All we need is to solve Eq. [60) and find messages $\left\{h_{i j}\right\}$ in a graph. Apart of the local magnetic moments, the Bethe-Peierls approximation allows one to find a spin correlation function and the free-energy. These formulas are given in Appendix A.

The Bethe-Peierls approach is exact for a treelike graph and the fully connected graph. It leads to the same equations as the cavity method and the exact recursion method (see Sec. VI.B). The Bethe-Peierls approach is approximate for graphs with loops due to spin correlations induced by loops. However, even in this case, it usually leads to remarkably accurate results. The approach can be improved by using the Kikuchi "cluster variation method" (Domb, 1960; Kikuchi, 1951; Yedidia et al., 2001).

How large are loop corrections to the Bethe-Peierls approximation? There is no clear answer on this important question. Several methods have recently been proposed for calculating loop corrections (Chertkov and Chernyak, 2006a. b; Montanari and Rizzo, 2005; Parisi and Slanina, 2006; Rizzo et al., 2006; Yedidia et al., 2001), however this problem is still unsolved.

a. Regular Bethe lattice. The Bethe-Peierls approach gives an exact solution of the ferromagnetic Ising model in an uniform magnetic field on a regular Bethe lattice with a coordination number $q$ (Baxter, 1982). In this case, all vertices and edges on the lattice are equivalent, therefore, $M_{i}=M$ and $h_{i j}=h$. From Eqs. (57) and (60), we obtain

$$
\begin{aligned}
M & =\tanh [\beta H+\beta q h], \\
\tanh \beta h & =\tanh \beta J \tanh [\beta H+\beta B h] .
\end{aligned}
$$

The parameter $B \equiv q-1$ on the right-hand side is the branching parameter.

At $H=0$, the model undergoes the standard second order phase transition at a critical point in which $B \tanh \beta J=1$. It gives the critical temperature

$$
T_{\mathrm{BP}}=2 J / \ln [(B+1) /(B-1)] .
$$

In the limit $q \gg 1$ the critical temperature $T_{\mathrm{MF}}$ tends to $T_{\mathrm{BP}}$, i.e., the simple mean-field approach Eq. (55) becomes exact in this limit. At the critical temperature $T=T_{\mathrm{BP}}$, the magnetic moment $M$ is a nonanalytic function of $H: M(H) \sim H^{1 / 3}$.

b. Fully connected graph. The Bethe-Peierls approximation is exact for the fully connected graph. For example, consider the spin-glass Ising model with random interactions $\left|J_{i j}\right| \propto N^{-1 / 2}$ on the graph (the SherringtonKirkpatrick model). The factor $N^{-1 / 2}$ gives a finite critical temperature. In the leading order in $N$, Eqs. (57) and (60) lead to a set of equations for magnetic moments $M_{i}$ :

$$
M_{i}=\tanh \left[\beta H+\beta \sum_{j} J_{i j} M_{j}-\beta^{2} \sum_{j} J_{i j}^{2} M_{i}\left(1-M_{j}^{2}\right)\right] .
$$

These are the TAP equations (Thouless et al., 1977) which are exact in the thermodynamic limit.

\section{Belief-propagation algorithm}

The belief-propagation algorithm is an effective numerical method for solving inference problems on sparse graphs. It was originally proposed by Pearl (1988) for treelike graphs. Among its numerous applications are computer vision problems, decoding of high performance turbo codes and many others, see Frey (1998); McEliece et al. (1998). Empirically it was found that it works surprisingly good even for graphs with loops. Yedidia et al. (2001) recently discovered that the belief-propagation algorithm actually coincides with the minimization of the Bethe free energy. This discovery renews interest in the Bethe-Peierls approximation and related methods Hartmann and Weigt, 2005; Mooij and Kappen, 2005; Pretti and Pelizzola, 2003). The recent progress in the survey propagation algorithm, which was proposed to solve some difficult combinatorial optimization problems, is a good example of interference between computer science and statistical physics (Braunstein and Zecchina, 2004; Mézard et al., 2002; Mézard and R. Zecchina, 2002).

In this section we give a physical interpretation of the belief-propagation algorithm in application to the Ising and other physical models on a graph. It enables us to find a general solution of an arbitrary physical model with discrete or continuous variables on a complex network. 
We start with the Ising model on a graph. Consider a spin $i$. Choose one of its nearest neighbors, say, a spin $j \in N(i)$. We define a parameter $\mu_{j i}\left(S_{i}\right)$ as probability to find spin $i$ in a state $S_{i}$ under the following conditions: (i) spin $i$ interacts only with spin $j$ while other neighboring spins are removed; (ii) an local magnetic field $H_{i}$ is zero. We normalize $\mu_{j i}\left(S_{i}\right)$ as follows: $\sum_{S_{i}= \pm 1} \mu_{j i}\left(S_{i}\right)=1$. For example, if $\mu_{j i}(+1)=1$ and $\mu_{j i}(-1)=0$, then the spin $j$ permits the spin state $S_{i}=+1$ and forbids the spin state $S_{i}=-1$. In the same way, we define probabilities $\mu_{n i}\left(S_{i}\right)$ for other neighboring spins $n \in N(i)$. We assume that the probabilities $\mu_{j i}\left(S_{i}\right)$ for all $j \in N(i)$ are statistically independent. Strictly speaking, this assumption holds true only in a treelike graph. For a graph with loops this approach is approximate. In the beliefpropagation algorithm the probabilities $\mu_{j i}\left(S_{i}\right)$ are traditionally called messages (do not mix with the messages in the Bethe-Peierls approach).

Let us search for an equilibrium state, using an iteration algorithm. We start from an initial set of nonequilibrium normalized probabilities $\left\{\mu_{j i}^{(0)}\left(S_{i}\right)\right\}$. Let us choose two neighboring vertices $i$ and $j$. Using the initial probabilities, we can calculate a probability to find a spin $j$ in a state $S_{j}$ under the condition that the state $S_{i}$ is fixed. This probability is proportional to the product of independent probabilities which determine the state $S_{j}$. First, we have the product of all incoming messages $\mu_{n j}^{(0)}\left(S_{j}\right)$ from nearest neighboring spins $n$ of $j$, except $i$, because its state is fixed. This is $\prod_{n \in N(j) \backslash i} \mu_{n j}^{(0)}\left(S_{j}\right)$. Second, we have a probabilistic factor $\exp \left(\beta H_{j} S_{j}\right)$ due to a local field $H_{j}$. Third, we have a probabilistic factor $\exp \left(\beta J_{i j} S_{i} S_{j}\right)$ due to the interaction between $i$ and $j$. Summing the total product of all these factors over two possible states $S_{j}= \pm 1$, we obtain a new probability:

$$
A \sum_{S_{j}= \pm 1} e^{\beta H_{j} S_{j}+\beta J_{i j} S_{i} S_{j}} \prod_{n \in N(j) \backslash i} \mu_{n j}^{(0)}\left(S_{j}\right)=\mu_{j i}^{\text {new }}\left(S_{i}\right),
$$

where $A$ is a normalization constant. This equation is the standard update rule of the belief-propagation algorithm. Its diagram representation is shown in Fig. 21, We assume that the update procedure converges to a fixed point $\mu_{j i}^{\text {new }}\left(S_{i}\right) \rightarrow \mu_{j i}\left(S_{i}\right)$. Sufficient conditions for convergence of the belief-propagation algorithm to a unique fixed point are derived in Ihler et al. (2005); Mooij and Kappen (2005). This fixed point determines an equilibrium state of the Ising model on a given graph. Indeed, we can write $\mu_{j i}\left(S_{i}\right)$ in a general form as follows:

$$
\mu_{j i}\left(S_{i}\right)=\exp \left(\beta h_{j i} S_{i}\right) /\left[2 \cosh \beta h_{j i}\right],
$$

where $h_{j i}$ is some parameter. Inserting Eq. (68) into Eq. (67), we obtain that the fixed point equation is exactly the recursion equation (60) in the Bethe-Peierls approach. This demonstrates a close relationship between the belief-propagation algorithm and the Bethe-Peierls approximation. Local magnetic moments and the Bethe free energy are calculated in Appendix B.

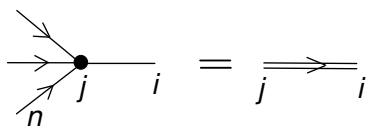

FIG. 21 Diagram representation of the belief-propagation update rule. Arrows show incoming messages to a vertex $j$. A factor $\exp \left(\beta H_{j} S_{j}\right)$ is shown as the closed circle. A solid line between $j$ and $i$ shows a factor $\exp \left(\beta J_{i j} S_{i} S_{j}\right)$. The double line is a new (outgoing) message from $j$ to $i$.

One can apply the belief-propagation algorithm to practically arbitrary physical model with discrete (Potts states) or continues (many component vectors) local parameters $x_{i}$. Let us introduce local energies $E_{i}\left(x_{i}\right)$ and pairwise interaction energies $E_{i j}\left(x_{i}, x_{j}\right)$. A generalized fixed point equation is

$$
A \sum_{x_{j}} e^{-\beta E_{j}\left(x_{j}\right)-\beta E_{i j}\left(x_{i}, x_{j}\right)} \prod_{n \in N(j) \backslash i} \mu_{n j}\left(x_{j}\right)=\mu_{j i}\left(x_{i}\right),
$$

where $A$ is a normalization constant. If $x_{i}$ is a continuous variable, then we integrate over $x_{j}$ instead of summing. In particular, one can show that for the Potts model this equation leads to the exact recursion equation (E2).

The belief-propagation algorithm was recently applied to study ferro- and antiferromagnetic, and spin-glass Ising models on the configuration model (Mooij and Kappen, 2005) and the Barabási-Albert growing network (Ohkubo et al., 2005).

\section{Annealed network approach}

In this subsection we describe an improved mean-field theory which accounts for heterogeneity of a complex network. Despite its simplicity, usually this approximation gives surprisingly good results in the critical region.

The main idea of the annealed network approach is to replace a model on a complex network by a model on a weighted fully connected graph. Let us consider the Ising model Eq. (54) on a graph with the adjacency matrix $a_{i j}$. We replace $a_{i j}$ by the probability that vertices $i$ and $j$ with degrees $q_{i}$ and $q_{j}$ are connected. For the configuration model, this probability is $q_{i} q_{j} / z_{1} N$, where $z_{1}=\langle q\rangle$. We obtain the Ising model on the fully connected graph:

$$
\mathcal{H}_{\mathrm{an}}=-\frac{1}{z_{1} N} \sum_{i<j} J_{i j} q_{i} q_{j} S_{i} S_{j}-\sum_{i} H_{i} S_{i}
$$

where $q_{i}$ plays the role of a "fitness" of vertex $i$. The resulting fully connected graph with these inhomogeneous fitnesses approximates the original complex network. Assuming that couplings $J_{i j}$ are finite and using exact equation (66), we find magnetic moments:

$$
M_{i}=\tanh \left[\beta H_{i}+\frac{\beta q_{i}}{z_{1} N} \sum_{j=1}^{N} J_{i j} q_{j} M_{j}\right] .
$$


Note that this set of equations is exact for the model Eq. (70) in the limit $N \rightarrow \infty$. For the ferromagnetic Ising model with $J_{i j}=J$ in zero field, i.e., $H_{i}=0$ for all $i$, the magnetic moment $M_{i}$ is given by

$$
M_{i}=\tanh \left[\beta J q_{i} M_{w}\right],
$$

where we introduced a weighted magnetic moment $M_{w} \equiv$ $\left(z_{1} N\right)^{-1} \sum_{j} q_{j} M_{j}$ which is a solution of the equation:

$$
M_{w}=\frac{1}{z_{1}} \sum_{q} P(q) q \tanh \left[\beta J q M_{w}\right] .
$$

Equations (72) and (73) were first derived by Bianconi (2002) for the Barabási-Albert network. They give an approximate mean-field solution of the ferromagnetic Ising model on an uncorrelated random complex network.

The effective model Eq. (70) undergoes a continues phase transition at a critical temperature $T_{c} / J=$ $z_{2} / z_{1}+1$ which approaches the exact critical temperature, Eq. (84), at $z_{2} / z_{1} \gg 1$. The annealed network approach gives a correct critical behavior of the ferromagnetic Ising model. It shows that at $T$ near $T_{c}$ the magnetic moment $M_{i} \propto q_{i} M_{w}$ for not too large degree $q_{i}$ in agreement with the microscopic results in Sec.VI.C.2 However this approach gives wrong results for a cooperative model on an original network with $z_{2} / z_{1} \rightarrow 1$, i.e., near the birth point of the giant connected component. It predicts a non-zero $T_{c}$ contrary to the exact one which tends to 0 . At $T=0$ the annealed network approximation gives the average magnetic moment $M=1$. The exact calculations in Sec. VI.C.2 give $M<1$ due to the existence of finite clusters with zero magnetic moment.

The annealed network approximation was used for studying the ferromagnetic Ising model with degreedegree dependent couplings Giuraniuc et al., 2005, 2006) and the random-field Ising model (Lee et al., 2006b). In Secs. VIII and X] we apply it to the ferromagnetic $X Y$ and Kuramoto models, respectively.

\section{B. The Ising model on a regular tree}

The Ising model Eq. (54) on a regular tree can be solved by using the exact recursion method developed for Bethe lattices and Cayley trees (Baxter, 1982). Recall that by definition, a Cayley tree is a finite tree while a Bethe lattice is infinite (see Sec. 【I.B). We will see that, even in the thermodynamic limit, thermodynamic properties of the ferromagnetic Ising model on a regular Cayley tree differ from those for a regular Bethe lattice.

\section{Recursion method}

Let us consider the ferromagnetic Ising model on a regular Cayley tree (see Fig. 1 in Sec. II.B). Any vertex can be considered as a root of the tree. This enables us to write a magnetic moment $M_{i}$ of spin $i$ and the partition function $Z$ as follows:

$$
\begin{aligned}
M_{i} & =\frac{1}{Z} \sum_{S_{i}= \pm 1} S_{i} e^{\beta H_{i} S_{i}} \prod_{j \in N(i)} g_{j i}\left(S_{i}\right), \\
Z & =\sum_{S_{i}= \pm 1} e^{\beta H_{i} S_{i}} \prod_{j \in N(i)} g_{j i}\left(S_{i}\right) .
\end{aligned}
$$

Here $g_{j i}\left(S_{i}\right)$ is a partition function of subtrees growing from vertex $j$, under the condition that the spin state $S_{i}$ is fixed:

$$
g_{j i}\left(S_{i}\right)=\sum_{\left\{S_{n}= \pm 1\right\}} \exp \left[\beta J_{i j} S_{i} S_{j}-\beta \mathcal{H}_{j \backslash i}\left(\left\{S_{n}\right\}\right)\right] .
$$

Here $\mathcal{H}_{j \backslash i}\left(\left\{S_{n}\right\}\right)$ is the interaction energy of spins, including spin $j$, on the subtrees except the edge $(i j)$. The advantage of a regular tree is that we can calculate the parameters $g_{j i}\left(S_{i}\right)$ at a given vertex $i$ by using the following recursion relation:

$g_{j i}\left(S_{i}\right)=\sum_{S_{j}= \pm 1} \exp \left[\beta J_{i j} S_{i} S_{j}+\beta H_{j} S_{j}\right] \prod_{m \in N(j) \backslash i} g_{m j}\left(S_{j}\right)$.

Note that this equation is equivalent to Eq. (67) at the fixed point within the belief-propagation algorithm. In order to shows this we introduce a parameter

$$
h_{j i} \equiv \frac{T}{2} \ln \left[g_{j i}(+1) / g_{j i}(-1)\right]
$$

and obtain $M_{i}=\tanh \left[\left(\beta H_{i}+\beta \sum_{j} h_{j i}\right)\right]$. According to the Bethe-Peierls approach in Sec. VI.A.1 the parameter $h_{j i}$ has the meaning of the additional field (message) created by vertex $j$ at nearest neighboring vertex $i$. These fields satisfy the recursion equation (60). In turn, Eq. (61) determines messages which go out boundary spins of a given tree. Starting from the boundary spins and using Eq. (60), we can calculate one by one all fields $h_{i j}$ on the Cayley tree and then find thermodynamic parameters of the Ising model.

\section{Spin correlations}

Using the recursion method, one can calculate the spin correlation function $\left\langle S_{i} S_{j}\right\rangle$ for two spins which are at a distance $\ell_{i j}$ from each other. We consider the general case when couplings $J_{i j}$ on a Cayley tree are arbitrary parameters. In zero field, $\left\langle S_{i} S_{j}\right\rangle$ is equal to a product of parameters $\tanh \beta J_{i j}$ along the shortest path connecting $i$ to $j$ :

$$
\left\langle S_{i} S_{j}\right\rangle=\prod_{m=0}^{\ell_{i j}-1} \tanh \beta J_{m, m+1} .
$$

Here the index $m$ numerates vertices on the shortest path, $m=0,1, \ldots \ell_{i j}$, where $m=0$ corresponds to 
vertex $i$ and $m=\ell_{i j}$ corresponds to vertex $j$ (Falk, 1975; Harris, 1975; Mukamel, 1974). This function coincides with a correlation function of an Ising spin chain (Bedeaux et al., 1970), and so spin correlations on a treelike graph have a one-dimensional character.

An even-spin correlation function $\left\langle S_{1} S_{2} \ldots S_{2 n}\right\rangle$ can also be calculated and presented as a product of pairwise correlation functions (Falk, 1975; Harris, 1975). Odd-spin correlation functions are zero in zero field.

\section{Magnetic properties}

The free energy of the ferromagnetic Ising model with $J_{i j}=J>0$ in zero magnetic field, $H=0$, on a regular Cayley tree was calculated by Eggarter (1974):

$$
F=-T L \ln [2 \cosh \beta J] .
$$

where $L$ is the number of edges. Moreover this is the exact free energy of an arbitrary tree with $L$ edges. $F$ is an analytic function of $T$. Hence there is no phase transition even in the limit $N \rightarrow \infty$ in contrast to a regular Bethe lattice. A magnetization is zero at all temperatures except $T=0$.

Muller-Hartmann and Zittartz (1974) revealed that the ferromagnetic Ising model on a regular Cayley tree with a branching parameter $B=q-1 \geq 2$ exhibits a new type of a phase transition which is seen only in the magnetic field dependence of the free energy. The free energy becomes a nonanalytic function of magnetic field $H>0$ at temperatures below the critical temperature $T_{\mathrm{BP}}$ given by Eq. (65):

$$
\begin{aligned}
F(T, H)= & F(T, H=0)+\sum_{l=1}^{\infty} a_{n}(T) H^{2 l} \\
& +A(T) H^{\kappa}+\mathcal{O}\left(H^{\kappa+1}\right),
\end{aligned}
$$

where $a_{n}(T)$ and $A(T)$ are temperature dependent coefficients. The exponent $\kappa$ depends on $T: \kappa=\ln B / \ln [B t]$, where $t \equiv \tanh \beta J$. It smoothly increases from 1 to $\infty$ as temperature varies from 0 to $T_{\mathrm{BP}}$, see Fig. 22, $F(T, H)$ is a continuous function of $H$ at $T=T_{\mathrm{BP}}$. All derivatives of $F$ with respect to $H$ are finite. Therefore, the phase transition is of the infinite order in contrast to the second order phase transition on a regular Bethe lattice (see Sec. VI.A.1). With decreasing $T$ below $T_{\mathrm{BP}}$, the singularity in $F$ is enhanced. The leading nonanalytic part of $F$ has a form $H^{2 l}|\ln H|$ at critical temperatures $T_{l}$ given by an equation $t B^{1-1 / 2 l}=1$ which leads to $T_{1}<T_{2}<\ldots<T_{\infty}=T_{\mathrm{BP}}$. The zero-field susceptibility $\chi(T)$ diverges as $\left(1-t^{2} B\right)^{-1}$ at $T=T_{1}$. Note that this divergence does not means the appearance of a spontaneous magnetization. Magnetic properties of the Ising model on a regular Cayley tree were studied in (Falk, 1975; Heimburg and Thomas, 1974; Matsuda, 1974; Melin et al., 1996; Stosic et al., 1998).

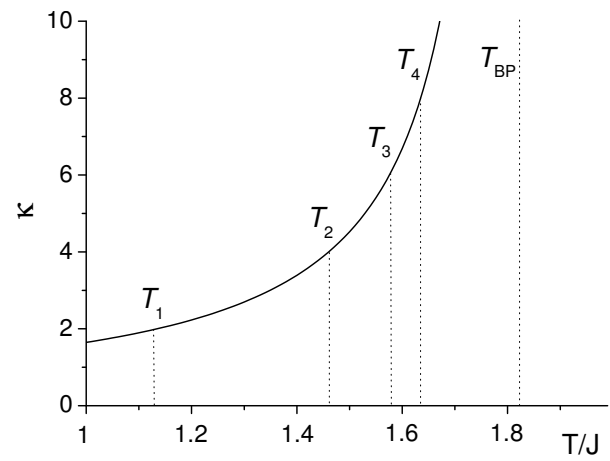

FIG. 22 Exponent $\kappa$ versus $T$ for the ferromagnetic Ising model on the regular Cayley tree with degree $q=3$. The critical temperatures $T_{l}$ are shown in dotted lines.

Insight into the origin of the critical points $T_{1}$ and $T_{\mathrm{BP}}$ may be gained by considering local magnetic properties of the Cayley tree. Let us apply a small local magnetic field $\Delta H_{i}$ on a vertex $i$. Due to ferromagnetic coupling between spins, this field induces a magnetic moment $\Delta \mathcal{M}(i)=\beta V(i) \Delta H_{i}$ in a region around $i$, where $V(i)$ is a so-called correlation volume which determines a size of likely ferromagnetic correlations around $i$ (see Sec. VI.C.4). An exact calculation of $V(i)$ shows that the correlation volume of the central spin diverges at $T=T_{\mathrm{BP}}$ in the infinite size limit $N \rightarrow \infty$. The central spin has long-ranged ferromagnetic correlations with almost all spins except for spins at a finite distance from the boundary. The correlation volume of a boundary spin diverges at a lower temperature $T=T_{1}<T_{\mathrm{BP}}$ simultaneously with the zero-field susceptibility $\chi(T)=$ $N^{-1} \sum_{i} \beta V(i)$. Therefore long-ranged spin correlations cover the whole system only at $T<T_{1}$.

A specific structure of the Cayley tree leads to the existence of numerous metastable states (Melin et al., 1996) which do not exist on a Bethe lattice. These states have a domain structure (see Fig. 231) and are stable with respect to single-spin flips. In order to reverse all spins in a large domain it is necessary to overcome an energy barrier which is proportional to the logarithm of the domain size. Therefore, a state with large domains will relax very slowly to the ground state. Melin et al. (1996) found that a glassy-like behavior appears at temperatures below a crossover temperature $T_{g}=2 \mathrm{~J} / \ln [\ln N / \ln B]$. Notice that $T_{g} \rightarrow 0$ as $N \rightarrow \infty$. However, $T_{g}$ is finite in a finite Cayley tree. Even if $N$ is equal to Avogadro's number $6.02 \times 10^{23}$, we obtain $T_{g} \approx 0.46 J$ at $q=3$. For comparison, $T_{\mathrm{BP}} \approx 1.8 \mathrm{~J}$ and $T_{1} \approx 1.1 \mathrm{~J}$. Large domains of flipped spins may arise at $T<T_{g}$. This leads to a non-Gaussian form of the magnetization distribution.

\section{The ferromagnetic Ising model on uncorrelated networks}

Here we show how strong is the influence of network topology on the critical behavior of the ferromagnetic 


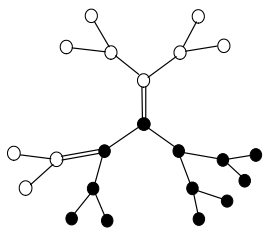

FIG. 23 Domains of flipped spins in the ferromagnetic Ising model on a regular Cayley tree. Filled and open circles represent spins up and down. Double lines shows "frustrated" edges connecting antiparallel spins.

Ising model. We will see that when increased network heterogeneity changes the critical behavior (the ferromagnetic phase transition becomes less sharp) and simultaneously increases the critical temperature. We also discuss spin correlations and finite-size effects.

\section{Derivation of thermodynamic quantities}

The microscopic theory of the ferromagnetic Ising model on uncorrelated random networks was developed by using the exact recursion method (Dorogovtsev et al., $2002 \mathrm{~b})$, which is equivalent to the Bethe-Peierls approximation, and the replica trick (Leone et al., 2002).

We consider the ferromagnetic Ising model, Eq. (54), with couplings $J_{i j}=J>0$ in uniform magnetic field $H_{i}=H$ within the Bethe-Peierls approach (see Sec. VI.A.1). In this approach, a thermodynamic state of the model is completely described by additional fields (messages) created by spins. In a complex network, due to intrinsic heterogeneity, the fields are random parameters. We introduce a distribution function $\Psi(h)$ of messages $h_{i j}: \Psi(h)=\left(1 / N z_{1}\right) \sum_{i, j} \delta\left(h-h_{i j}\right)$, where $N z_{1}$ is the normalization constant. If we assume the selfaverageness, then, in the limit $N \rightarrow \infty$, the average over a graph is equivalent to the average over a statistical network ensemble.

A self-consistent equation for $\Psi(h)$ follows from the recursion equation (60) (see also Fig. 20 in Sec. VI.A.1):

$$
\begin{aligned}
\Psi(h)= & \sum_{q} \frac{P(q) q}{z_{1}} \int \delta\left(h-T \tanh ^{-1}[\tanh \beta J \times\right. \\
& \left.\left.\tanh \left(\beta H+\beta \sum_{m=1}^{q-1} h_{m}\right)\right]\right) \prod_{m=1}^{q-1} \Psi\left(h_{m}\right) d h_{m} .
\end{aligned}
$$

This equation assumes, first, that all incoming messages $\left\{h_{m}\right\}$ are statistically independent. This assumption is valid for an uncorrelated random network. Second, an outgoing message $h$ is sent along a chosen edge by a vertex of degree $q$ with the probability $P(q) q / z_{1}$. If we know $\Psi(h)$, we can find the free energy and other thermodynamic parameters (see Appendix A). For example, the average magnetic moment is

$$
M=\sum_{q} P(q) \int \tanh \left(\beta H+\beta \sum_{m=1}^{q} h_{m}\right) \prod_{m=1}^{q} \Psi\left(h_{m}\right) d h_{m} .
$$

The replica trick (Leone et al., 2002) leads to the same equations (see Appendix C).

\section{Phase transition}

In the paramagnetic phase at zero field $H=0$, equation (82) has a trivial solution: $\Psi(h)=\delta(h)$, i.e., all messages are zero. A non-trivial solution (which describes a ferromagnetically ordered state) appears below a critical temperature $T_{c}$ :

$$
T_{c}=2 J / \ln \left(\frac{z_{2}+z_{1}}{z_{2}-z_{1}}\right)
$$

This is the exact result for an uncorrelated random network (Dorogovtsev et al., 2002b; Leone et al., 2002))

The critical temperature $T_{c}$ can be found from a "naive" estimate. As we have noted in Sec. VI.A.1, the critical temperature $T_{\mathrm{BP}}$, Eq. (65), is determined by the branching parameter rather than the mean degree. In a complex network, the branching parameter fluctuates from edge to edge. The average branching parameter $B$ may remarkably differ from the mean degree $z_{1}$. For the configuration model, inserting the average branching parameter $B=z_{2} / z_{1}$ into Eq. (65), we obtain Eq. (84). If the parameter $z_{2}$ tends to $z_{1}$, then $T_{c} \rightarrow 0$. It is not surprising, because at the percolation threshold we have $z_{2}=z_{1}$, and the giant connected component disappear.

A general analytical solution of Eq. (82) for the distribution function $\Psi(h)$ is unknown. A correct critical behavior of the Ising model at $T$ near $T_{c}$ can be found by using an "effective medium" approximation:

$$
\sum_{m=1}^{q-1} h_{m} \approx(q-1) \bar{h}+\mathcal{O}\left(q^{1 / 2}\right)
$$

where $\bar{h} \equiv \int h \Psi(h) d h$ is the average field which can be found self-consistently (Dorogovtsev et al., 2002b). This approximation takes into account the most "dangerous" highly connected spins in the best way. The ansatz (85) is equivalent to the approximation $\Psi(h) \sim \delta(h-\bar{h})$ (Leone et al., 2002). At lower temperatures a finite width of $\Psi(h)$ becomes important.

The ferromagnetic Ising model on uncorrelated random networks demonstrates three classes of universal critical behavior:

(i) the standard mean-field critical behavior in networks with a finite fourth moment $\left\langle q^{4}\right\rangle$ (scalefree networks with the degree distribution exponent $\gamma>5)$ 
TABLE I Critical behavior of the magnetization $M$, the specific heat $\delta C$, and the susceptibility $\chi$ in the Ising model on networks with a degree distribution $P(q) \sim q^{-\gamma}$ for various values of exponent $\gamma . \tau \equiv 1-T / T_{c}$.

\begin{tabular}{cccc}
\hline \hline & $M$ & $\delta C\left(T<T_{c}\right)$ & $\chi$ \\
\hline$\gamma>5$ & $\tau^{1 / 2}$ & jump at $T_{c}$ & \\
$\gamma=5$ & $\tau^{1 / 2} /\left(\ln \tau^{-1}\right)^{1 / 2}$ & $1 / \ln \tau^{-1}$ & $\tau^{-1}$ \\
$3<\gamma<5$ & $\tau^{1 /(\gamma-3)}$ & $\tau^{(5-\gamma) /(\gamma-3)}$ & \\
\hline$\gamma=3$ & $e^{-2 T /(q)}$ & $T^{2} e^{-4 T /\langle q\rangle}$ & $T^{-1}$ \\
$2<\gamma<3$ & $T^{-1 /(3-\gamma)}$ & $T^{-(\gamma-1) /(3-\gamma)}$ & $T^{2}$ \\
\hline \hline
\end{tabular}

(ii) a critical behavior with non-universal critical exponents depending on a degree distribution in networks with divergent $\left\langle q^{4}\right\rangle$, but a finite second moment $\left\langle q^{2}\right\rangle$ (scale-free networks with $3<\gamma \leq 5$ );

(iii) an infinite order phase transition in networks with a divergent second moment $\left\langle q^{2}\right\rangle$, but a finite mean degree $\langle q\rangle$ (scale-free networks with $2<\gamma \leq 3$ ).

The corresponding critical exponents $\left(M \sim \tau^{\beta}, \delta C \sim\right.$ $\left.\tau^{-\alpha}, \chi \sim \tau^{-\tilde{\gamma}}\right)$ are reported in the Table【 The evolution of the critical behavior with increasing heterogeneity is shown schematically in Fig. 24. Notice that the Ising model on a regular random network demonstrates the standard mean-field critical behavior in the infinite size limit (Scalettar, 1991). The corresponding exact solution is given in Sec. VI.A.1.a

The conventional scaling relation between the critical exponents takes place at $\gamma>3$ :

$$
\alpha+2 \beta+\tilde{\gamma}=2 \text {. }
$$

Interestingly, the magnetic susceptibility $\chi$ has a universal critical behavior with the exponent $\tilde{\gamma}=1$ when $\left\langle q^{2}\right\rangle<\infty$, i.e., at $\gamma>3$. This agrees with the scaling relation $\tilde{\gamma} / \nu=2-\eta$ if we insert the standard mean-field exponents: $\nu=1 / 2$ and the Fisher exponent $\eta=0$ (see Sec. IX.B. When $2<\gamma \leq 3$, the susceptibility $\chi$ has a paramagnetic temperature dependence, $\chi \propto 1 / T$, at temperatures $T \gtrsim J$ despite the system is in the ordered state.

At $T<T_{c}$ the ferromagnetic state is strongly heterogeneous because the magnetic moment $M_{i}$ fluctuates from vertex to vertex. The ansatz Eq. (85) enables us to find an approximate distribution function of $M_{i}$ :

$$
Y(M) \equiv \frac{1}{N} \sum_{i=1}^{N} \delta\left(M-M_{i}\right) \approx \frac{P(q(M))}{\beta \bar{h}\left(1-M^{2}\right)},
$$

where the function $q(M)$ is a solution of an equation $M(q)=\tanh [\beta \bar{h} q]$. Near $T_{c}$, low-degree vertices have a small magnetic moment, $M(q) \sim q\left|T_{c}-T\right|^{1 / 2} \ll 1$, while hubs with degree $q>T / \bar{h} \gg 1$ have $M(q) \sim 1$. The function $Y(M)$ is shown in Fig. 25. Note that the distribution of magnetic moments in scale-free networks is more inhomogeneous than in the Erdős-Rényi graphs. Moreover $Y(M)$ diverges at $M \rightarrow 1$. A local magnetic

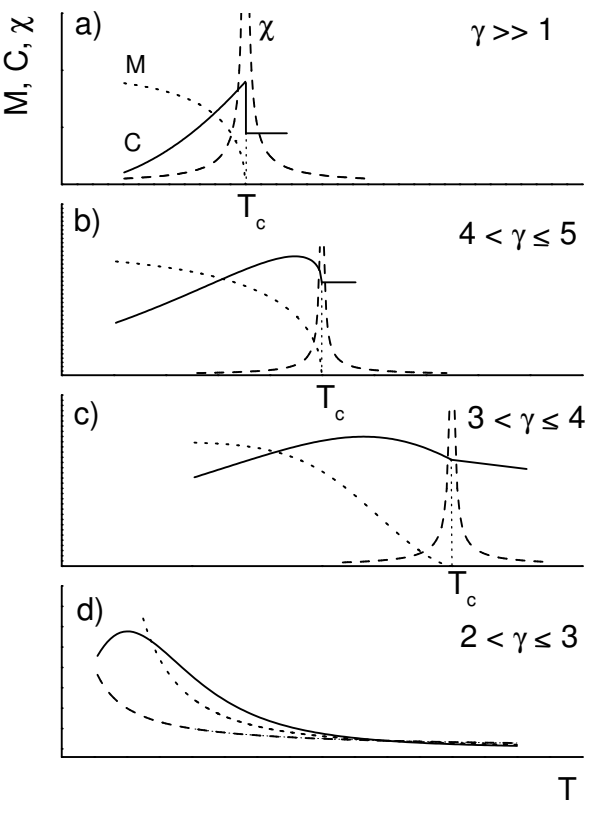

FIG. 24 Schematic representation of the critical behavior of the magnetization $M$ (dotted lines), the magnetic susceptibility $\chi$ (dashed lines), and the specific heat $C$ (solid lines) for the ferromagnetic Ising model on uncorrelated random networks with a degree distribution $P(q) \sim q^{-\gamma}$. (a) $\gamma \gg 1$, the standard mean-field critical behavior. A jump of $C$ disappears when $\gamma \rightarrow 5$. (b) $4<\gamma \leqslant 5$, the ferromagnetic phase transition is of second order. (c) $3<\gamma \leqslant 4$, the transition becomes of higher order. (d) $2<\gamma \leqslant 3$, the transition is of infinite order, and $T_{c} \rightarrow \infty$ as $N \rightarrow \infty$.

moment depends on its neighborhood. In particular, a magnetic moment of a spin, neighboring a hub may differ from a moment of a spin surrounded by low-degree vertices. Studies of these correlations are at the very beginning (Giuraniuc et al., 2006).

In the ground state $(T=0, H=0)$, an exact distribution function $\Psi(h)$ converges to a function with two delta peaks:

$$
\Psi(h)=x \delta(h)+(1-x) \delta(h-J),
$$

where the parameter $x$ is determined by an equation describing percolation in networks (see Sec. III.B.1). Equation (88) tells us that in the ground state, spins, which be- 


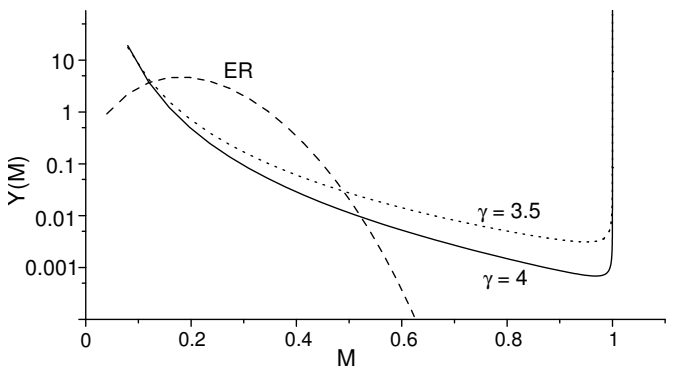

FIG. 25 Distribution function $Y(M)$ of magnetic moments $M$ in the ferromagnetic Ising model on the Erdös-Rényi graph with mean degree $z_{1}=5$ (dashed line) and scale-free graphs with $\gamma=4$ and 3.5 (solid and dotted lines) at $T$ close $T_{c}$, $\beta \bar{h}=0.04$.

long to a finite cluster, have zero magnetic moment while spins in a giant connected component have magnetic moment 1 because non-zero fields acts on these spins. The average magnetic moment is $M=1-\sum_{q} P(q) x^{q}$. This is exactly the size of the giant connected component of the network.

\section{Finite-size effects}

When $2<\gamma \leqslant 3$, a dependence of $T_{c}$ on the size $N$ is determined by the finite-size cutoff $q_{\text {cut }}(N)$ of the degree distribution in Sec. II.E.4. We obtain

$$
\begin{array}{ll}
T_{c}(N) \approx \frac{z_{1} \ln N}{4}, & \text { at } \gamma=3, \\
T_{c}(N) \approx \frac{(\gamma-2)^{2} z_{1} q_{\text {cut }}^{3-\gamma}(N)}{(3-\gamma)(\gamma-1)}, & \text { at } 2<\gamma<3
\end{array}
$$

(Bianconi, 2002; Dorogovtsev et al., 2002b; Leone et al., 2002). These estimates agree with the numerical simulations of Aleksiejuk et al. (2002); Herrero (2004). Notice that Herrero used the cutoff $q_{\text {cut }}(N) \sim N^{1 / \gamma}$ which leads to $T_{c} \sim N^{z}$ with the exponent $z=(3-\gamma) / \gamma$.

\section{Ferromagnetic correlations}

Let us consider spin correlations in the ferromagnetic Ising model in the paramagnetic state. Recall that the correlation length $\xi$ of spin correlations in the Ising model on a finite-dimensional lattice diverges at a critical point of a continuous phase transition. In contrast, in an uncorrelated random complex network, the correlation length $\xi$ is finite at any temperature. Indeed, according to Eq. (79), the correlation function $C(\ell)=\left\langle S_{i} S_{j}\right\rangle$ decays exponentially with distance $\ell \equiv \ell_{i j}: C(\ell)=e^{-\ell / \xi}$, where the coherence length $\xi \equiv 1 /|\ln \tanh \beta J| \neq 0$ at non-zero temperature. Moreover, spin correlations have a one-dimensional character despite a complex network is an infinite-dimensional system. Strictly speaking, this is valid at distances $\ell<\bar{\ell}(N) \sim \ln N$ when a network is treelike.

In complex networks, the so called correlation volume rather than $\xi$ plays a fundamental role (see also Sec. III.B.3). We define a correlation volume $V(i)$ around a spin $i$ as follows:

$$
V(i) \equiv \sum_{j=1}^{N} a_{i j}\left\langle S_{i} S_{j}\right\rangle
$$

It determines the size of likely ferromagnetic fluctuations around the spin. In the paramagnetic phase, $V(i)$ is expressed through local network characteristics: $V(i)=$ $\sum_{\ell=0}^{\infty} z_{\ell}(i) t^{\ell}$, where $t \equiv \tanh \beta J$, and $z_{\ell}(i)$ is the number of vertices which are at a distance $\ell$ from vertex $i$, and $z_{0}(i) \equiv 1$.

It is obvious that a correlation volume around a high degree vertex (hub) is larger than the one around a poorly connected vertex. In a scale-free network, hubs may form a highly connected cluster (the rich-club phenomenon (Colizza et al., 2006; Zhou and Mondragón, 2004)). A region of likely ferromagnetic correlations around the rich-club may be very large. It grows with decreasing $T$, absorbing small clusters of correlated spins. The ErdösRényi network is more homogeneous than a scale-free network with the same average degree. At high temperatures there are many small clusters of ferromagnetically correlated spins. With decreasing $T$ small clusters merge together, forming larger clusters.

The average correlation volume $\bar{V}$ is related with the total magnetic susceptibility:

$$
\bar{V} \equiv \frac{1}{N} \sum_{i=1}^{N} V(i)=\sum_{\ell=0}^{\infty} z_{\ell} t^{\ell}=T \chi
$$

where $z_{\ell}$ is the average number of $\ell$-th nearest neighbors of a vertex on a given network: $z_{\ell}=N^{-1} \sum_{i} z_{\ell}(i)$. The average correlation volume $\bar{V}$ diverges as $\ln N$ in the critical point of a continuous phase transition. The condition of divergence of the series in Eq. (92) leads to the equation: $B \tanh \beta_{c} J=1$, where $B \equiv \lim _{\ell \rightarrow \infty} \lim _{N \rightarrow \infty}\left[z_{\ell}\right]^{1 / \ell}$ is the average branching parameter of the network. This criterion for the critical point is valid for any treelike network (Lyons, 1989), including networks with degreedegree correlations, growing networks etc. $B=1$ corresponds to the point of the birth of the giant connected component. At $B<1$ a network consists of finite clusters, the correlation volume is finite at all $T$, and there is no phase transition.

Using Eq. (92), we can calculate $\chi$ in the paramagnetic phase. In the configuration model of uncorrelated random networks, we have $z_{\ell}=z_{1}\left(z_{2} / z_{1}\right)^{\ell-1}$. This gives

$$
T \chi=\bar{V}=1+\frac{z_{1} t}{1-z_{2} t / z_{1}} .
$$

So $\chi$ diverges as $\left|T-T_{c}\right|^{-1}$. 
Equation (79) for the correlation function $\left\langle S_{i} S_{j}\right\rangle$ is not valid for scale-free networks with $2<\gamma<3$ due to numerous loops. How do spin correlations decay in this case? Dorogovtsev et al. (2005) found that in these networks the pair correlation function $\left\langle S_{i} S_{j}\right\rangle$ between the second and more distant neighbors vanishes in the limit $N \rightarrow \infty$. Only pair correlations between nearest neighbors are observable in this limit.

\section{Degree-dependent interactions}

Giuraniuc et al. (2005, 2006) studied analytically and numerically a ferromagnetic Ising model on a scale-free complex network with a topology dependent coupling: $J_{i j}=J z_{1}^{2 \mu}\left(q_{i} q_{j}\right)^{-\mu}$, where a constant $J>0, \mu$ is a tunable parameter, $q_{i}$ and $q_{j}$ are degrees of neighboring vertices $i$ and $j$. The authors demonstrated that the critical behavior of the model on a scale-free network with degree distribution exponent $\gamma$ is equivalent to the critical behavior of the ferromagnetic Ising model with a constant coupling $J$ on a scale-free network with renormalized degree distribution exponent $\gamma^{\prime}=(\gamma-\mu) /(1-\mu)$. Therefore the critical exponents can be obtained, replacing $\gamma$ by $\gamma^{\prime}$ in Table @] Varying $\mu$ in range $[2-\gamma, 1]$ allows us to explore the whole range of the universality classes represented in Table I. For example, the ferromagnetic Ising model with $J_{i j}=J$ on a scale-free network with $\gamma=3$ undergoes an infinite order phase transition while the model with the degree dependent coupling for $\mu=1 / 2$ undergoes a second order phase transition with the critical behavior corresponding to $\gamma^{\prime}=5$.

\section{The Ising model on small-world networks}

The phase transition in the ferromagnetic Ising model on small-world networks strongly resembles that in the percolation problem for these nets, Sec. III.G. This system was extensively studied by Barrat and Weigt (2000), Gitterman (2000), Pekalski (2001), and many other researchers. Here we mostly discuss small-world networks based on one-dimensional lattices, with a fraction $p$ of shortcuts. Let us estimate the critical temperature $T_{c}(p)$ assuming for the sake of simplicity only nearest-neighbor interactions in the one-dimensional lattice. The reader may easily see that if $p$ is small, this network has a locally tree-like structure. At small $p$, the mean branching parameter in this graph is $B=1+c p+O\left(p^{2}\right)$, where $c$ is some model dependent constant. Substituting $B$ into Eq. (65), we arrive at

$$
T_{c}(p) \sim \frac{J}{|\ln p|},
$$

where $J$ is the ferromagnetic coupling. Barrat and Weigt (2000) arrived at this result using the replica trick. Exact calculations of Lopes et al. (2004) confirmed this formula.
Far from the critical temperature, the thermodynamic quantities of this system are close to those of the $d$ dimensional substrate lattice. However, in the vicinity of the critical temperature the ordinary mean-field picture is valid. Two circumstances naturally explain these traditional mean-field features. (i) In the interesting range of small $p$, the small-world networks effectively have a locally tree-like structure (short loops due the lattice are not essential). (ii) The small-world networks have rapidly decreasing degree distributions. As we have explained, this architecture leads to the traditional mean-field picture of critical phenomena. The region of temperatures around $T_{c}(p)$, where this mean-field picture is realized, is narrowed as $p$ decreases. Lopes et al. (2004) obtained the specific heat as a function of temperature and $p$ and showed that its jump at the critical point approaches zero as $p \rightarrow 0$. Roy and Bhattacharjee (2006) demonstrated numerically that the Ising model on the WattsStrogatz network is self-averaging in the limit $N \rightarrow \infty$, i.e., the average over this ensemble is equivalent to the average over a single Watts-Strogatz network. With increasing network size $N$, the distributions of the magnetization, the specific heat and the critical temperature of the Ising model in the ensemble of different realizations of the Watts-Strogatz network approach the $\delta$-function. The size dependence of these parameters agrees with the finite scaling theory in Sec. IX.B.

Hastings (2003) investigated the Ising model on the $d$-dimensional small-world. He found that for any $d$, the shift of the critical temperature is $T_{c}(p)-T_{c}(p=$ $0) \sim p^{1 / \tilde{\gamma}}$, where $\tilde{\gamma}$ is the susceptibility exponent at $p=0, \chi(T, p=0) \sim\left|T-T_{c}(0)\right|^{-\tilde{\gamma}}$. Compare this shift with the similar shift of the percolation threshold in the same network, Sec. III.G. Simulations of Herrero (2002); Zhang and Novotny (2006) confirmed this prediction.

In their simulations, Jeong et al. (2003) studied the Ising model with specific interactions placed on the ordinary one-dimensional small-world network. In their system the ferromagnetic interaction between two neighboring spins, say, spins $i$ and $j$, is $|i-j|^{-\alpha}$. $|i-j|$ is a distance measured along the chain. Surprisingly, a phase transition was revealed only at $\alpha=0$, no long-range order for $\alpha>0$ was observed at any non-zero temperature. Chatterjee and Sen (2006) performed numerical simulations of the ferromagnetic Ising model placed on a one-dimensional small-world network, where vertices, say, $i$ and $j$, are connected by a shortcut with probability $\sim|i-j|^{-\alpha}$ (Kleinberg's network, see Sec. II.I). They observed a phase transition at least at $\alpha<1$. In both these studies, the small sizes of simulated networks made difficult to arrive at reliable conclusions. On the other hand, these two systems were not studied analytically.

\section{E. Spin glass transition on networks}

Despite years of efforts, the understanding of spin glasses is still incomplete. The nature of the spin- 
glass state is well understood for the infinite-range Sherrington-Kirkpatrick model (Binder and Young, 1986; Mézardet al., 1987). The basic property of the spin-glass model is that a huge number of pure thermodynamic states with non-zero local magnetic moments $M_{i}$ spontaneously emerge below a critical temperature. This corresponds to the replica symmetry breaking.

Investigations of a spin-glass Ising model on treelike networks began very soon after the discovery of spin glasses. Viana and Bray (1985) proposed the so called dilute Ising spin-glass model which is equivalent to the Ising model on the Erdös-Rényi graph (the reader will find a review of early investigations in Mézard and Parisi (2001)). Most of studies considered a spin-glass on random regular and Erdős-Rényi networks. A spin-glass on the Watts-Strogatz and scale-free networks only recently drew attention.

Here we first review recent studies of the spin-glass Ising model on complex networks. Then we consider a pure antiferromagnetic Ising model, which becomes a spin-glass when placed on a complex network, and discuss relationships of this model with famous NP-complete problems (MAX-CUT and vertex cover).

\section{The Ising spin glass}

The spin-glass state arises due to frustrations. The nature of frustrations in the Sherrington-Kirkpatrick model and a spin-glass model on a finite dimensional lattice is clear. On the other hand, for an uncorrelated random network, the nature of frustrations is not so clear because such a network has a treelike structure in the thermodynamic limit. How do frustrations appear in this case? In order to answer this question we recall that locally tree-like networks usually have numerous long loops of typical length $O(\ln N)$, see Sec. II.G. It turns out that frustrations in a network are due to these long loops.

Two main methods are used to study the spin-glass Ising model on a random network. These are the replica trick and the cavity method (Mézard and Parisi, 2001). Early investigations of a spin glass on a Bethe lattice assumed that there is only one pure thermodynamic state, and the replica symmetry is unbroken. This assumption led to unphysical results such as, for example, a negative specific heat. The order parameter of the Sherrington-Kirkpatrick model is an overlap $\left\langle S_{\alpha} S_{\beta}\right\rangle$ between spins of two replicas $\alpha$ and $\beta$. The spin-glass Ising model on a random network requires multi-spin overlaps $\left\langle S_{\alpha} S_{\beta} S_{\gamma}\right\rangle,\left\langle S_{\alpha} S_{\beta} S_{\gamma} S_{\delta}\right\rangle$ and higher Goldschmidt and Dominicis, 1990; Kim et al., 2005; Mottishaw, 1987; Viana and Bray, 1985). This makes this model more complex.

Many evidences have been accumulated indicating that a spin-glass state may exist in the spin-glass Ising model on a Bethe lattice (Mézard and Parisi, 2001). It means that this model has many pure thermodynamic states at low temperatures. In order to obtain a complete description of a spin-glass state it is necessary to solve the recursion equations (60) and find the distribution function $\Psi_{\alpha}(h)$ of the messages for every pure state $\alpha$. It is a difficult mathematical problem which is equivalent to search for a solution with replica symmetry breaking. In order to find an approximate solution, a one step replica-symmetry breaking approximation was developed (Castellani et al., 2005; Mézard and Parisi, 2001; Pagnani et al., 2003). This approximation assumes that a space of pure states has a simple cluster structure (a set of clusters). Numerical simulations of the spin-glass Ising model on a random regular network demonstrated that this approximation gives better results than the replica symmetric solution. A similar result was obtained for the Watts-Strogatz network (Nikoletopoulos et al., 2004). Unfortunately the space of pure states is probably more complex, and a solution with a complete replica symmetry breaking is necessary.

The phase diagram of the Ising spin glass on the ErdösRényi graphs was studied by Castellani et al. (2005); Kanter and Sompolinsky (2000); Kwon and Thouless (1988), and Hase et al. (2006). The diagram looks like the phase diagram of the Sherrington-Kirkpatrick model. The exact critical temperature of the spin-glass transition, $T_{\mathrm{SG}}$, on a treelike complex network can be found without the replica trick. The criterion of this transition is the divergence of the spin-glass susceptibility:

$$
\chi_{\mathrm{SG}}=\frac{1}{N} \sum_{i=1}^{N} \sum_{j=1}^{N}\left\langle S_{i} S_{j}\right\rangle^{2}
$$

Using Eq. (79) for the correlation function $\left\langle S_{i} S_{j}\right\rangle$, we find that $\chi_{\mathrm{SG}}$ diverges at a critical temperature $T_{\mathrm{SG}}$ determined by the following equation:

$$
B \int \tanh ^{2}\left(\beta_{\mathrm{SG}} J_{i j}\right) P\left(J_{i j}\right) d J_{i j}=1,
$$

where $B$ is the average branching parameter.

If the distribution function $P\left(J_{i j}\right)$ is asymmetric, and the mean coupling $\bar{J}=\int J_{i j} P\left(J_{i j}\right) d J_{i j}$ is larger than a critical value, then a ferromagnetic phase transition occurs at a higher critical temperature $T_{c}$ than $T_{\mathrm{SG}}$. The criterion of the ferromagnetic phase transition is the divergence of the magnetic susceptibility $\chi$ :

$$
B \int \tanh \left(\beta_{c} J_{i j}\right) P\left(J_{i j}\right) d J_{i j}=1 .
$$

In a multicritical point, we have $T_{c}=T_{\mathrm{SG}}$. Equations (96) -(97) generalize the results obtained by the replica trick and others methods for regular random graphs, the Erdös-Rényi networks, and the static and configuration models of uncorrelated complex networks (Baillie et al., 1995; Kim et al., 2005; Mooij and Kappen, 2005; Ostilli, 2006a; b; Thouless, 1986; Viana and Bray, 1985).

It is well-known that if $\bar{J}$ exceeds a critical value, the Sherrington-Kirkpatrick model at low temperatures undergoes a phase transition from a ferromagnetic state 
into a so called mixed state in which ferromagnetism and spin-glass order coexist. The coexistence of ferromagnetism and spin-glass order in the spin-glass Ising model on a random regular graph with degree $q$ was considered by Castellani et al. (2005); Liers et al. (2003). Castellani et al. (2005) studied a zero-temperature phase diagram of the spin-glass Ising model with a random coupling $J_{i j}$ which takes values $\pm J$ with probabilities $(1 \pm \rho) / 2$. They found that at $\rho$ exceeding a critical value $\rho_{c}(q)$ the spin-glass Ising model is in a replica symmetric ferromagnetic state. For $\rho<\rho_{c}(q)$, the replica symmetric state becomes unstable. The system goes into a mixed state with a broken replica symmetry. In particular, for degree $q=3, \rho_{c}(q=3)=5 / 6$. At $q \gg 1$, $\rho_{c}(q) \sim \ln q / \sqrt{q}$. The one-step symmetry breaking solution showed that the mixed state exists in a range $\rho_{\mathrm{F}}<\rho<\rho_{c}(q)$. At $\rho<\rho_{\mathrm{F}}$ the ground state is a nonmagnetic spin-glass state. Liers et al. (2003) studied numerically a spin-glass model with a Gaussian coupling $J_{i j}$. They did not observe a mixed state in contrast to Castellani et al. (2005).

A strong effect of the network topology on the spinglass phase transition was recently revealed by Kim et al. (2005) in the Ising spin-glass model with $J_{i j}= \pm J$ on an uncorrelated scale free network. These authors used a replica-symmetric perturbation approach of Viana and Bray (1985). It turned out that in a scale-free network with $3<\gamma<4$, the critical behavior of the spinglass order parameter at $T$ near $T_{\mathrm{SG}}$ differs from the critical behavior of the Sherrington-Kirkpatrick model and depends on $\gamma$. For the paramagnetic-ferromagnetic phase transition, a deviation from the standard critical behavior takes place at $\gamma<5$ similarly to the ferromagnetic Ising model in Sec. VI.C.2. Critical temperatures of the ferromagnetic and spin-glass phase transitions approach infinity in the thermodynamic limit at $2<\gamma<3$. These transitions become of infinite order.

\section{The antiferromagnetic Ising model and MAX-CUT problem}

The antiferromagnetic (AF) Ising model becomes nontrivial on a complex network. As we will see, the model is a spin glass. We here also discuss a mapping of the ground state problem onto the MAX-CUT problem.

Consider the pure AF model on a graph:

$$
E=\frac{J}{2} \sum_{i, j} a_{i j} S_{i} S_{j}
$$

where $J>0$. The search for the ground state is equivalent to coloring a graph in two colors (colors correspond to spin states $S= \pm 1$ ) in such a way that no two adjacent vertices have the same color. Let us first consider a bipartite network, that is a network without odd loops. It is obvious that this network is 2-colorable. The ground state energy of the AF model on a bipartite graph is $E_{0}=-J L$, where $L$ is the total number of edges in the graph. An uncorrelated complex network with a giant

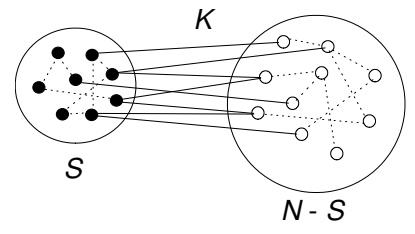

FIG. 26 Partition of vertices in a graph into two sets consisting of $S$ and $N-S$ vertices, and $K$ edges (solid lines) in the cut. Dotted lines show edges inside the sets.

connected component cannot be colored with 2 colors due to numerous odd loops. So the ground state energy, $E_{0}$, of the AF model on a random graph is higher than $-J L$ due to frustrations produced by odd loops.

The ground state problem can be mapped to the MAXCUT problem which belongs to the class of NP-complete optimization problems. Let us divide vertices of a graph (of $N$ vertices and $L$ edges) into two sets in such a way that the number $K$ of edges which connect these sets is maximum, see Fig. 26. If we define spins at vertices in one set as spins up and spins in the other set as spins down, then the maximum cut gives a minimum energy $E_{0}$ of the AF model. Indeed, $K$ edges between two sets connect antiparallel spins and give a negative contribution $-J K$ into $E_{0}$. The remaining $L-K$ edges connect parallel spins and give a positive contribution $J(L-K)$. The ground state energy, $E_{0}=J(L-2 K)$, is minimum when $K$ is maximum.

The maximum cut of the Erdős-Rényi graph with high probability is

$$
K_{c} \equiv \max K=L / 2+A N \sqrt{z_{1}}+o(N)
$$

for mean degree $z_{1}>>1$ (Coppersmith et al., 2004; Kalapala and Moore, 2002). Here $A$ is a constant with lower and upper bounds $0.26<A<\sqrt{\ln 2} / 2 \approx 0.42$. Recall that $L=z_{1} N / 2$. The estimation of $K_{c}$ is given in Appendix D, Thus the ground state energy is

$$
E_{0} / N=-2 J A \sqrt{z_{1}} .
$$

The fraction of "frustrated" edges, i.e., edges which connect "unsatisfied" parallel spins, is $\left(L-K_{c}\right) / L=$ $1 / 2-2 A / \sqrt{z_{1}}$. Thus almost half of edges are frustrated. We found that this result is valid not only for classical random graphs but also for arbitrary uncorrelated random network.

Interestingly, the lower bound of the ground state energy Eq. (100) is quite similar to the lower bound for the ground state energy of the random energy model introduced by Derrida (1981). This model approximates to spin-glass in any dimensions. Replacing the mean degree $z_{1}$ in Eq. (100) by degree of a $D$-dimensional cubic lattice, $2 D$, we obtain the ground state energy of Derrida's model: $E_{0} / N=-J \sqrt{2 D \ln 2}$. (We are grateful to $\mathrm{M}$. Ostilli for attracting our attention to this fact.)

Despite the seeming simplicity, the pure AF model on complex networks is not well studied yet. We assume that this model is the usual spin glass. On the 
other hand, the analysis of Mooij and Kappen (2005) revealed an antiferromagnetic phase transition in the model on an uncorrelated random network at a critical point $\left(z_{2} / z_{1}\right) \tanh \beta J=1$, i.e., at the critical temperature $T_{\mathrm{BP}}$ in Eq. (84). If this result is correct, then, as temperature decreases, the AF model may undergo a phase transition from an antiferromagnetic state into a spin-glass state. The structure of pairwise spin correlations in this system is non-trivial. The correlations between two spins separated by distance $\ell$ are characterized by their average value $\bar{C}(\ell)=z_{\ell}^{-1} \sum_{i j}\left\langle S_{i} S_{j}\right\rangle \delta_{\ell, \ell_{i j}}$. We expect that at least for locally tree-like networks, the spin correlations are antiferromagnetic at all distances smaller than the mean intervertex separation $\bar{\ell}(N)$. These correlations should be present in the spin-glass phase and even in some range of temperatures above the spin-glass transition. Antiferromagnetic correlations of this kind were observed in numerical simulations by Bartolozzi et al. (2006).

Holme et al. (2003) used the antiferromagnetic Ising model to study the bipartivity of real-world networks (professional collaborations, on-line interactions and so on). In order to measure the bipartivity, they proposed to put the AF model on the top of the network and calculate a fraction of edges between spins with opposite signs in the ground state. We have explained that this procedure is equivalent to finding the maximum cut of the graph. The larger is this fraction the closer is the network to bipartite. Measuring bipartivity allows one to reveal the bipartite nature of seemingly one-partite networks. Note that only their one-mode projections are usually studied, while most of real-world networks are actually multipartite.

\section{Antiferromagnet in a magnetic field, the hard-core gas model, and vertex covers}

Here we discuss relations between an antiferromagnetic Ising model, the hard-core gas model and the vertex cover problem on classical random graphs. On complex networks these problems are poorly studied.

a. The vertex cover problem. This problem is one of the basic NP-complete optimization problems. A vertex cover of a graph is a set of vertices with the property that every edge of the graph has at least one endpoint which belongs to this set. In general, there are many different vertex covers of a graph. We look for a vertex cover of a minimum size, see Fig. 27. Weigt and Hartmann (2000) proposed a vivid picture for this problem: "Imagine you are director of an open-air museum situated in a large park with numerous paths. You want to put guards on crossroads to observe every path, but in order to economize cost you have to use as few guards as possible."

Let us find size of a minimum vertex cover of the ErdősRényi graph of $N$ vertices, $L=z_{1} N / 2$ edges and mean

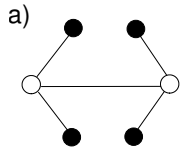

b)

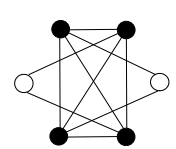

FIG. 27 Vertex cover of a graph. a) Open circles form a minimum vertex cover of the graph. Every edge has at least one endpoint which belongs to the vertex cover. The closed circles form the maximum independent set of the graph. b) The complement of the same graph (we add the missing edges and remove the already existing edges). Closed circles form the maximum clique.

degree $z_{1}$. We denote the number of vertices in a vertex cover as $N_{v c}=x N$. The parameter $x$ can be interpreted as the probability that a randomly chosen vertex is covered, i.e., it belongs to the vertex cover. An edge can be between every pair of vertices with the same probability. So the probability that a randomly chosen edge connects two vertices which do not belong to the vertex cover is $(1-x)^{2}$. With the conjugate probability $1-(1-x)^{2}=2 x(1-x)$, an edge has at least one covered endpoint. There are $\left(\begin{array}{c}N \\ N_{v c}\end{array}\right)$ ways to choose $N_{v c}$ vertices from $N$ vertices. Only a small fraction of the partitions, $[2 x(1-x)]^{L}$, are vertex covers. Thus the number of possible vertex covers is

$$
\mathfrak{N}_{v c}(x)=\left(\begin{array}{c}
N \\
N_{v c}
\end{array}\right)[2 x(1-x)]^{L} \equiv e^{N \Xi(x)} .
$$

Using the estimate Eq. (D3), we obtain

$$
\Xi(x)=-(1-x) \ln (1-x)-x \ln x+\frac{z_{1}}{2} \ln [2 x(1-x)] .
$$

The threshold fraction $x_{c}$ is determined by the condition: $\Xi\left(x_{c}\right)=0$. It gives $x_{c}\left(z_{1}\right) \approx 1-2 \ln z_{1} / z_{1}+O\left(\ln \ln z_{1}\right)$ at $z_{1} \gg 1$. The exact asymptotics was found by Frieze (1990):

$$
x_{c}\left(z_{1}\right)=1-\frac{2}{z_{1}}\left(\ln z_{1}-\ln \ln z_{1}-\ln 2+1\right)+o\left(\frac{1}{z_{1}}\right) .
$$

At $x<x_{c}$, with high probability there is no vertex cover of size $x N<x_{c} N$, while at $x>x_{c}$ there are exponentially many different covers of size $x N>x_{c} N$. The appearance of many vertex covers looks like a phase transition which occurs at the threshold parameter $x=x_{c}$.

The exact threshold $x_{c}\left(z_{1}\right)$ and the number of minimum vertex covers were calculated for the Erdős-Rényi graph by using a statistical mechanics analysis of ground state properties of a hard-core model (see below) and the replica method. The replica symmetric solution gives an exact result in the interval $1<z_{1} \leqslant e$ :

$$
x_{c}\left(z_{1}\right)=1-\frac{2 W\left(z_{1}\right)+W\left(z_{1}\right)^{2}}{2 z_{1}},
$$

where $W(x)$ is the Lambert-function defined by an equation $W \exp W=x$ (Weigt and Hartmann, 2000), see also 
Weigt and Zhou (2006). The same result was derived by Bauer and Golinelli (2001a b) , using the leaf algorithm. Note that the giant connected component of the ErdösRényi graph disappear at $z_{1}<1$. The presence of the replica symmetry indicates that in the interval $1<z_{1} \leqslant e$ the degeneracy of the minimum vertex covers is trivial in the following sense. One can interchange a finite number of covered and uncovered vertices in order to receive another minimum vertex cover. Many non-trivial minimum vertex covers appear at mean degrees $z_{1}>e$. The replica symmetry is broken and Eq. (104) is not valid. For this case the threshold $x_{c}\left(z_{1}\right)$ and the degeneracy of the minimum vertex cover were calculated by using the onestep replica symmetry breaking in Weigt and Hartmann (2000, 2001); Zhou (2003). Minimum vertex covers form a single cluster at $z_{1} \leqslant e$, while they are arranged in many clusters at $z_{1}>e$. As a result, the typical running time of an algorithm for finding a vertex cover at $z_{1} \leqslant e$ is polynomial while the time grows exponentially with the graph size at $z_{1}>e$ (Barthel and Hartmann, 2004).

The vertex cover problem on correlated scale-free networks was studied by Vázquez and Weigt (2003). It turned out that increase of likewise degree-degree correlations (assortative mixing) increases the computational complexity of this problem in comparison with an uncorrelated scale-free network having the same degree distribution. If the assortative correlations exceed a critical threshold, then many nontrivial vertex covers appear.

Interestingly, the minimum vertex cover problem is essentially equivalent to another NP-hard optimization problem-the maximum clique problem. Recall that a clique is a subset of vertices in a given graph such that each pair of vertices in the subset are linked. In order to establish the equivalence of these two optimization problems, it is necessary to introduce the notion of the complement or inverse of a graph. The complement of a graph $G$ is a graph $\bar{G}$ with the same vertices such that two vertices in $\bar{G}$ are connected if and only if they are not linked in $G$. In order to find the complement of a graph, we must add the missing edges, and remove the already existing edges. One can prove that vertices, which do not belong to the maximum clique in $\bar{G}$, form the minimum vertex cover in $G$ (see Fig. 27).

A generalization of the vertex cover problem to hypergraphs can be found in Mézard and Tarzia (2007).

b. The hard-core gas model. Let us treat uncovered vertices as particles, so that we assign a variable $\nu=1$ for uncovered and $\nu=0$ for covered vertices. Hence there are $\sum_{i} \nu_{i}=N-N_{v c}$ particles on the graph. We also introduce a repulsion between particles such that only one particle can occupy a vertex (the exclusion principle). A repulsion energy between two nearest neighboring particles is $J>0$. Then we arrive at the so called hard-core gas model with the energy

$$
E=\frac{J}{2} \sum_{i, j} a_{i j} \nu_{i} \nu_{j}
$$

where $a_{i j}$ are the adjacency matrix elements. If the number of particles is not fixed, and there is a mass exchange with a thermodynamic bath, then we add a chemical potential $\mu>0$. This results in the Hamiltonian of the hard-core gas model: $\mathcal{H}=E-\mu \sum_{i=1}^{N} \nu_{i}$.

In the ground state of this model, particles occupy vertices which do not belong to a minimum vertex cover. Their number is equal to $\left(1-x_{c}\right) N$, where $x_{c}$ is the fraction of vertices in the minimum vertex cover. The ground state energy is $E_{0}=0$ because configurations in which two particles occupy two nearest neighboring vertices, are energetically unfavorable. In other words, particles occupy the maximum subset of vertices in a given graph such that no two vertices are adjacent. In graph theory, this subset is called the maximum independent set (see Fig. 27). Unoccupied vertices form the minimum vertex cover of the graph. Thus finding the minimum vertex cover (or equivalently, the maximum independent set) of a graph is equivalent to finding the maximum clique of the complement of this graph.

The ground state of the hard-core model is degenerate if there are many minimum vertex covers (or equivalently, many maximum independent sets). The reader may see that searching for the ground state is exactly equivalent to the minimum vertex cover problem.

c. Antiferromagnet in a random field. Let us consider the following antiferromagnetic Ising model (Zhou, 2003, 2005):

$$
E=\frac{J}{2} \sum_{i, j} a_{i j} S_{i} S_{j}-\sum_{i=1}^{N} S_{i} H_{i}+J L .
$$

Here $J>0, H_{i}=-J q_{i}$ is a degree dependent local field, where $q_{i}$ is degree of vertex $i$. $L$ is the number of edges in a graph. The negative local fields force spins to be in the state -1 , however the antiferromagnetic interactions compete with these fields.

Consider a spin $S_{i}$ surrounded by $q_{i}$ nearest neighbors $j$ in the state $S_{j}=-1$. The energy of this spin is

$$
\left(J \sum_{j \in N(i)} S_{j}-H_{i}\right) S_{i}=0 \times S_{i}=0
$$

in any state $S_{i}= \pm 1$. Therefore this spin is effectively free. Positions of "free" spins on a graph are not quenched. If one of the neighboring spins flips up, then the state $S_{i}=-1$ becomes energetically favorable.

Let us apply a small uniform magnetic field $\mu, 0<$ $\mu \ll J$. At $T=0$, all "free" spins are aligned along $\mu$, i.e., they are in the state +1 . One can prove that the spins $S=+1$ occupy vertices which belong to the maximum independent set, while the spins $S=-1$ occupy the minimum vertex cover of a given graph. For this let us make the transformation $S_{i}=2 \nu_{i}-1$, where $\nu_{i}=0,1$ for spin states $\mp 1$, respectively. Then the antiferromagnetic model Eq. (106) is reduced to the hard-core gas model where the external field $\mu$ corresponds to the chemical 


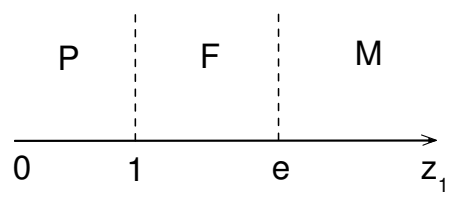

FIG. 28 Phase diagram of the antiferromagnetic Ising model Eq. (106) at $T=0$. P, F and $\mathrm{M}$ denote paramagnetic, ferromagnetic and mixed (spin-glass) phases, respectively. At mean degree $z_{1}>e$, in the mixed phase, ferromagnetism and spin-glass order coexist.

potential of the particles. The energy of the degenerate ground state is $E_{0}=0$. All these pure states have the same energy $E_{0}=0$, the same average magnetic moment $M=1-2 x_{c}$ but correspond to different non-trivial minimum vertex covers.

The exact mapping of the AF model Eq. (106) onto the vertex cover problem leads to the zero-temperature phase diagram shown in Fig. 28. The model is in a paramagnetic state at small degree $0<z_{1}<1$ because in this case the network is below the percolation threshold and consists of finite clusters. Above the percolation threshold, at $1<z_{1}<e$, the ground state is ferromagnetic with an average magnetic moment $M=1-2 x_{c}\left(z_{1}\right)$, where $x_{c}\left(z_{1}\right)$ is given by Eq. (104). The replica symmetry is unbroken at $z_{1}<e$. Many pure states appear spontaneously and the replica symmetry is broken at $z_{1}>e$. In this case the $\mathrm{AF}$ model is in a mixed phase in which ferromagnetism and spin-glass order coexist. At $z_{1} \gg 1$ the magnetic moment $M$ is determined by $x_{c}\left(z_{1}\right)$ from Eq. (103).

\section{F. The random-field Ising model}

The random-field Ising model is probably one of the simplest models showing a dramatic influence of a quenched disorder (random fields) on a collective behavior of a system with an exchange interaction (Imry and Ma, 1975; Lacour-Gavet and Toulouse, 1974). Despite its simplicity, the random-field model was an object of intensive and controversial investigations during the last three decades. The energy of this model is

$$
E=-\frac{J}{2} \sum_{i, j} a_{i j} S_{i} S_{j}-H \sum_{i} S_{i}-\sum_{i} H_{i} S_{i}
$$

where $J>0, H$ is a uniform field, and $H_{i}$ is a random field. In most cases, the distribution function of the random field is either Gaussian,

$$
P_{\mathrm{RF}}\left(H_{i}\right)=\frac{1}{\sqrt{2 \pi} \sigma} \exp \left[-\frac{H_{i}^{2}}{2 \sigma^{2}}\right]
$$

or bimodal,

$$
P_{\mathrm{RF}}\left(H_{i}\right)=\frac{1}{2} \delta\left(H_{i}-H_{0}\right)+\frac{1}{2} \delta\left(H_{i}+H_{0}\right) .
$$

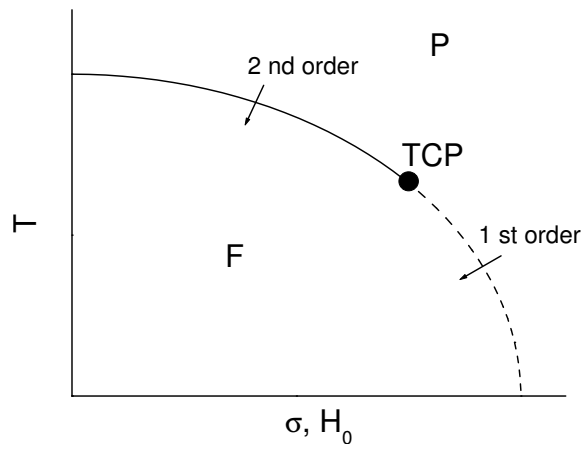

FIG. 29 Phase diagram of the random-field model on the fully connected graph. For the Gaussian distribution, the phase transition from the para- $(\mathrm{P})$ to ferromagnetic $(\mathrm{F})$ phase is of second order in the $T-\sigma$ plane. For the bimodal distribution, there is a tricritical point (TCP) in the $T-H_{0}$ plane. The phase transition is of second order (solid line) above TCP and first order (dashed line) below TCP.

The parameters $\sigma$ and $H_{0}$ characterize a strength of random fields.

The search for the ground state of the random-field model on a graph is related with a famous optimization problem of a maximum flow through the graph (Picard and Ratliff (1975); see also Hartmann and Weigt $(2005))$. This problem belongs to the class $\mathrm{P}$, that is it may be solved in time bound by a polynomial in the graph size.

\section{Phase diagram}

The random-field model is exactly solved on the fully connected graph (all-to-all interaction) (Aharony, 1978; Schneider and Pytte, 1977). In this case we replace the coupling $J$ by $J / N$ in Eq. (108). The average magnetic moment is

$$
M=\int \tanh \left[\beta\left(J M+H+H_{i}\right)\right] P_{\mathrm{RF}}\left(H_{i}\right) d H_{i} .
$$

For the Gaussian distribution, the phase transition from the para- to ferromagnetic state is a mean-field second order phase transition. Sufficiently strong random fields suppress the phase transition at $\sigma>\sigma_{c}=J[2 / \pi]^{1 / 2}$, and the system is in a disordered state at all $T$. The phase diagram of the random-field model with the bimodal distribution of random field is shown in Fig. 29, Bruinsma (1984) found that the random-field model with the bimodal distribution on a regular Bethe lattice has a rich ground state structure.

\section{Hysteresis on a fully connected graph}

The random-field model demonstrates a peculiar hysteresis phenomena at $T=0$ which may be relevant 
for understanding out-of-equilibrium phenomena in many complex systems (Sethna et al., 2001).

We start with a physical picture of the hysteresis which is valid for any network. Let all spins be in the state -1 . This initial state corresponds to an applied field $H=-\infty$. An adiabatic increase of the magnetic field results in a series of the so called discrete Barkhausen jumps (avalanches) of a finite size (Perkovic et al., 1995; Sethna et al., 1993). A spin avalanche can be initiated by a single spin flip. Indeed, if the total magnetic field $H+H_{i}$ at vertex $i$ becomes larger than the energy of the ferromagnetic interaction of the spin with neighboring spins, then the spin turns up. This spin flip can stimulate flips of neighboring spins, if they are energetically favorable. In turn the neighboring spins may stimulate flips of their neighbors and so on. As a result we observe an avalanche. If $H$ is smaller than a critical field $H_{c}(\sigma)$, then the average avalanche size is finite. At $H=H_{c}(\sigma)$ a macroscopic avalanche takes place, and the magnetization has a jump $\Delta M$.

The exact properties of the hysteresis on the fully connected graph at $T=0$ were found by Sethna et al. (1993). The dependence of the magnetization $M$ on $H$ along a hysteresis loop follows from Eq. (111):

$$
M=2 \int_{-M J-H}^{\infty} P_{\mathrm{RF}}\left(H_{i}\right) d H_{i}-1 .
$$

The analysis of this equation for the Gaussian distribution of random fields shows that the critical field $H_{c}(\sigma)$ is non-zero at small strengths $\sigma<\sigma_{c}$. There is no hysteresis at a sufficiently large strength of the random field, $\sigma>\sigma_{c}=J[2 / \pi]^{1 / 2}$, see Fig. 30. The magnetization has a universal scaling behavior near the critical point $\left(\sigma_{c}\right.$, $\left.H_{c}\left(\sigma_{c}\right)\right)$ :

$$
M(r, h)=\left|\sigma-\sigma_{c}\right|^{\beta} G\left(h /\left|\sigma-\sigma_{c}\right|^{\beta \delta}\right),
$$

where $h=H-H_{c}\left(\sigma_{c}\right) . \quad \beta=1 / 2$ and $\delta=3$ are the mean-field critical exponents. $G(x)$ is a scaling function.

\section{Hysteresis on a complex network}

Another approach applied to zero-temperature hysteresis on the random regular graph was developed by Dhar et al. (1997). Here we generalize this approach to the configuration model of an uncorrelated random network with a given degree distribution $P(q)$.

As above, we suppose that all spins are in the initial state -1 at $H=-\infty$. Then the applied field is adiabatically increased. Let $P^{*}$ be the conventional probability that if a spin at an end of a randomly chosen edge is down, then for the other end spin, it will be energetically favorable to flip up. $P^{*}$ satisfies the equation:

$$
P^{*}=\sum_{q} \frac{P(q) q}{z_{1}} \sum_{n=0}^{q-1}\left(\begin{array}{c}
q-1 \\
n
\end{array}\right)\left[P^{*}\right]^{n}\left[1-P^{*}\right]^{q-1-n} p_{n}(H)
$$
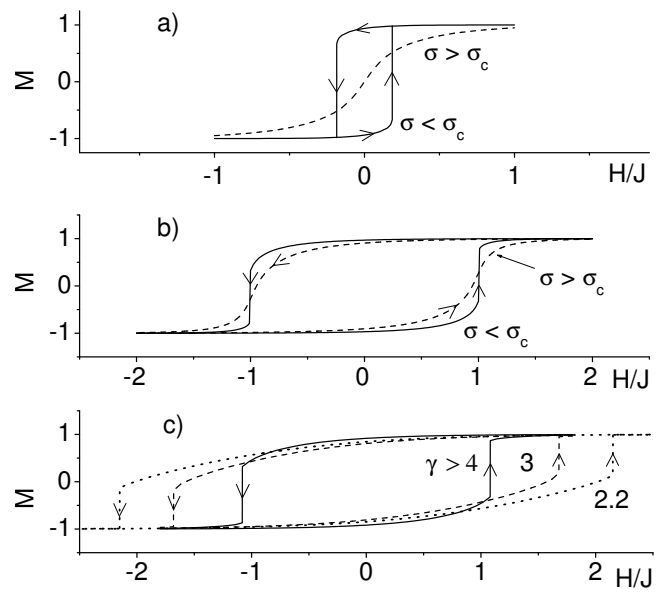

FIG. 30 Hysteresis in the ferromagnetic Ising model with Gaussian random fields (magnetization $M$ versus $H$ ). (a) Fully connected graph: solid line, $\sigma=0.5<\sigma_{c}$; dashed line, $\sigma \geqslant \sigma_{c}$. (b) Random regular network with degree $q=4$ : solid line, $\sigma=1.7<\sigma_{c}$, dashed line, $\sigma=2 \geqslant \sigma_{c}$. (c) Uncorrelated scale-free networks for $\sigma=1.7$ : solid line, $\gamma \geq 4,\langle q\rangle \approx 4$; dashed line, $\gamma=3,\langle q\rangle \approx 4$; dotted line, $\gamma=2.2,\langle q\rangle=5.3$.

where $q$ is vertex degree. The $n$-th term in the sum is the probability that $n$ neighbors of a spin turn up simultaneously with the spin while the other $q-n-1$ neighboring spins remain in the state -1 . The parameter

$$
p_{n}(H) \equiv \int_{-H+(q-2 n) J}^{\infty} P_{\mathrm{RF}}\left(H_{i}\right) d H_{i}
$$

is the probability to find a vertex with a random field $H_{i}>-H+(q-2 n) J$. Knowing $P^{*}$, we can calculate the fraction of spins which turn up at an applied field $H$ :

$$
N_{\uparrow}(H)=\sum_{q} P(q) \sum_{n=0}^{q}\left(\begin{array}{l}
q \\
n
\end{array}\right)\left[P^{*}\right]^{n}\left[1-P^{*}\right]^{q-n} p_{n}(H) .
$$

It gives the magnetization: $M(H)=2 N_{\uparrow}(H)-1$. Note that Eqs. (114) and (116) resemble Eqs. (31) and (30) describing the $k$-core architecture of networks.

Hysteresis was only studied in detail for a random regular network (all vertices have the same degree, i.e., $\left.P(q)=\delta_{q, k}\right)$, see Fig. 30. In this case there is hysteresis without a jump of the magnetization if the strength $\sigma$ of Gaussian random fields is larger than a critical strength $\sigma_{c}$, in contrast to the fully connected graph where hysteresis disappears at $\sigma>\sigma_{c}$. The critical field $H_{c}$ of the magnetization jump does not depend on $q>3$. Monte Carlo simulations of the random-field model on a random regular network made by Dhar et al. (1997) confirmed this analytical approach. A numerical solution of Eqs. (115) and (116) shows that the random-field model on uncorrelated scale-free networks has a similar hysteresis behavior (see Fig. 30). Note that the critical field $H_{c}$ 
depends on the degree distribution exponent $\gamma$ only when $2<\gamma<4$.

A similar hysteresis phenomenon was found numerically in the antiferromagnetic Ising model on growing scale-free and Erdős-Rényi networks within zero-temperature field-driven dynamics of spins (Hovorka and Friedman, 2007; Malarz et al., 2007; Tadić et al., 2005). It was shown that the network topology influences strongly properties of hysteresis loops. In this model, it is the network inhomogeneity that plays the role of disorder similar to the random fields.

\section{The random-field model at $T=0$}

Critical properties of the random-field model at $T=0$ on scale-free networks were studied numerically and analytically by Lee et al. (2006b) by using a mean-field approximation which is equivalent to the annealed network approximation in Sec. VI.A.3 These authors found that a critical behavior near a phase transition from a disordered state into the ferromagnetic state depends on degree distribution exponent $\gamma$. If the distribution function of random fields is concave at $H_{i}=0$ (i.e., $P_{\mathrm{RF}}^{\prime \prime}\left(H_{i}=0\right)<0$, similar to the Gaussian distribution) then the spontaneous magnetization $M$ emerges below a critical strength $\sigma_{c}: M \propto\left|\sigma_{c}-\sigma\right|^{\beta}$, where $\beta(\gamma>5)=1 / 2, \beta(3<\gamma \leqslant 5)=1 /(\gamma-3)$. In the case of the convex distribution function, i.e., $P_{\mathrm{RF}}^{\prime \prime}\left(H_{i}=0\right)>0$, the phase transition is of the first order at all $\gamma>3$. When $2<\gamma \leqslant 3$, the random-field model is in the ferromagnetic state for an arbitrary strength and an arbitrary distribution function of random fields. This effect is quite similar to the effect found in the ferromagnetic Ising and Potts models in Secs. VI.C.2, VII, and IX).

Son et al. (2006) proposed to use the random-field model as a tool for extracting a community structure in complex networks. In sociophysics, the random-field Ising model is used for describing the emergence of a collective opinion.

\section{G. The Ising model on growing networks}

In this section we assume that a spin system on a growing network approaches equilibrium much faster than the network changes, and the adiabatic approximation works. So we discuss the following circle of problems: a network is grown up to an infinite size and then the Ising model is placed on it.

\section{Deterministic graphs with BKT-like transitions}

As is natural, the use of deterministic graphs dramatically facilitates the analysis of any problem. Surprisingly,

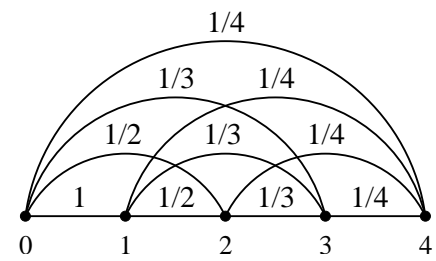

FIG. 31 The deterministic fully connected graph (Costin et al., 1990), which is equivalent to the asymmetric annealed network. The values of the Ising coupling are shown on the edges.

very often results obtained in this way appear to be qualitatively similar to conclusions for models on random networks. Various graphs similar to those shown in Fig. 4allow one to effectively apply the real space renormalization group technique. For example, Andrade and Herrmann (2005) studied the Ising model on the graph shown in Fig. 4(c) - "the Apollonian network" — and observed features typical for the Ising model on random scale-free network with exponent $\gamma<3$.

More interestingly, the Ising model on some deterministic graphs shows the BKT-like singularities which was already discovered in the 1990s by Costin et al. (1990) and Costin and Costin (1991). In network context, their model was studied in Bauer et al. (2005). This network substrate is an asymmetric annealed network, which is actually an annealed version of the random recursive graph. Vertices are labelled $i=0,1,2, \ldots, t$, as in a growing network. Each vertex, say vertex $i$ have a single connection of unit strength to "older" vertices. One end of this edge is solidly fixed at vertex $i$, while the second end frequently hops at random among vertices $0,1, \ldots, i-1$, which just means the specific asymmetric annealing. The resulting network is equivalent to the fully connected graph with a specific large scale inhomogeneity of the coupling (see Fig. 31).

The ferromagnetic Ising model on this network is described by the Hamiltonian

$$
\mathcal{H}=-\sum_{0 \leq i<j \leq t} \frac{s_{i} s_{j}}{j}-\sum_{i=0}^{t} H_{i} s_{i}
$$

where $H_{i}$ are local magnetic fields. The mean-field theory, exact for this Hamiltonian, indicates the presence of a phase transition in this system. Figure 32 shows an inhomogeneous distribution of the magnetization $m(i)$ over the network. Only in the normal phase, without field, $m(i)=0$. Otherwise, the oldest spin turns out to be strictly directed, $m(i=0)=1$, and the profile is non-analytic: $m(i) \cong 1-\operatorname{const}(i / t)^{2 / T}$. Earlier, Coulomb and Bauer (2003) observed a resembling effect studying a giant connected component in random growing networks. The full magnetization $M(T)$ demon- 


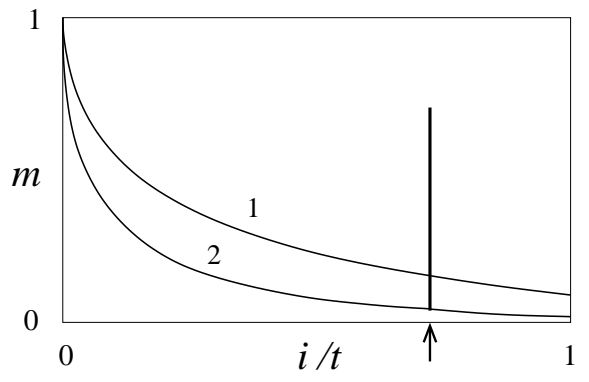

FIG. 32 The magnetization profile for the ferromagnetic Ising model on the graph shown in Fig. 31 labels vertices starting from the "oldest" one, and $t$ is the network size. Curve 1 is valid both for $T<T_{c}$ with an arbitrary homogeneous applied field $H$ and for $T>T_{c}, H \neq 0$. Curve 2 describes the profile when an external field is applied to a single spin, while $T>$ $T_{c}$. The arrow indicates the point of application of the local magnetic field. The mean magnetic moment of this vertex is very distinct from others.

strates the BKT-kind behavior near the phase transition:

$$
M(T) \propto \exp \left(-\frac{\pi}{2} \sqrt{\frac{T_{c}}{T_{c}-T}}\right) .
$$

Note that the BKT singularity, Eq. (118), and the specific non-analyticity of $m(i)$ at $i=0$ are closely related.

The distribution of the linear response, $\sum_{i} \partial m(i) /\left.\partial H_{j}\right|_{H=0}$, to a local magnetic field, which may be also called the distribution of correlation volumes, in this model is very similar to the size distribution of connected components in growing networks with the BKT-like transition. It has a power-law decay in the whole normal phase. Exactly the same decay has the distribution of correlations $\partial m(i) /\left.\partial H_{j}\right|_{H=0}$ in this phase (Khajeh et al., 2007).

We may generalize the inhomogeneity of the interaction in the Hamiltonian to a power law, $\propto j^{-\alpha}$, with an arbitrary exponent. (For brevity, we omit the normalization - the sum of the coupling strengths must grow proportionally to the size of the network.) One may show that in this model the BKT-singularity exists only when $\alpha=1$. For $\alpha>1$, phase ordering is absent at any nonzero temperature as in the one-dimensional Ising model, and for $0<\alpha<1$, there is a quite ordinary second order transition.

Let us compare this picture with the well-studied ferromagnetic Ising model for a spin chain with regular longrange interactions $\propto|i-j|^{-\alpha}$ (see, e.g., Luijten and Blöte (1997)). In this model, (i) for $\alpha>2, T_{c}=0$, similarly to the one-dimensional Ising model; (ii) at $\alpha=2$, there is a transition resembling the BKT one; (iii) for $1<\alpha<2$, there is a transition at finite $T_{c}$. The reader may see that in both models, there exist boundary values of exponent $\alpha$, where BKT-kind phenomena take place. In very simple terms, these special values of $\alpha$ play the role of lower critical dimensions. (Recall that the BKT transitions in solid state physics occur only at a lower critical dimension.) These associations show that the BKT singularities in these networks are less strange and unexpected than one may think at first sight.

Khajeh et al. (2007) solved the $q$-state Potts model on this network and, for all $q \geq 1$ arrived at results quite similar to the Ising model, i.e., $q=2$. Recall that $q=$ 1 corresponds to the bond percolation model, and that the traditional mean-field theory on lattices gives a first order phase transition if $q>2$. Thus, both the first and the second order phase transitions transformed into the BKT-like one on this network.

Hinczewski and Berker (2006) found another deterministic graph, on which the Ising model shows the BKTlike transition, so that this singularity is widespread in evolving networks with large-scale inhomogeneity.

\section{The Ising model on growing random networks}

There is still no analytical solution of the Ising model on growing random networks. Aleksiejuk et al. (2002) and their numerous followers simulated the Ising model on the very specific Barabási-Albert network, where degree-degree correlations are virtually absent. So, the resulting picture is quite similar to the Ising model on an uncorrelated scale-free network with degree distribution exponent $\gamma=3$. In general, the growth results in a wide spectrum of structural correlations, which may dramatically change the phase transition.

Based on known results for the percolation (the onestate Potts model), see Sec. III.F we expect the following picture for the Ising model on recursive growing graphs. If each new vertex has a single connection, the recursive graph is a tree, and so the ferromagnetic ordering takes place only at zero temperature. Now let a number of connections of new vertices be greater than 1 , so that these networks are not trees. (i) If new vertices are attached to randomly chosen ones, there will be the BerezinskiiKosterlitz-Thouless critical singularity. (ii) If the mechanism of the growth is the preferential attachment, then the critical feature is less exotic, more similar to that for uncorrelated networks.

\section{THE POTTS MODEL ON NETWORKS}

The Potts model is related to a number of outstanding problems in statistical and mathematical physics (Baxter, 1982; Wu, 1982). The bond percolation and the Ising model are only particular cases of the $p$ state Potts model. The bond percolation is equivalent to one-state Potts model (Fortuin and Kastelevn (1972); Kasteleyn and Fortuin (1969), see also Lee et al. (2004c)). The Ising model is exactly the two-state Potts model. Here we first look at critical properties of the Potts model and then consider its applications for coloring a random graph and for extracting communities. 


\section{A. Solution for uncorrelated networks}

The energy of the Potts model with $p$ states is

$$
E=-\frac{1}{2} \sum_{i, j} J_{i j} a_{i j} \delta_{\alpha_{i}, \alpha_{j}}-H \sum_{i} \delta_{\alpha_{i}, 1}
$$

where $\delta_{\alpha, \beta}=0,1$ if $\alpha \neq \beta$ and $\alpha=\beta$, respectively. Each vertex $i$ can be in any of $p$ states: $\alpha_{i}=1,2, \ldots, p$. The "magnetic field" $H>0$ distinguishes the state $\alpha=1$. The $\alpha$-component of the magnetic moment of vertex $i$ is defined as follows:

$$
M_{i}^{(\alpha)}=\frac{p\left\langle\delta_{\alpha_{i}, \alpha}\right\rangle-1}{p-1} .
$$

In the paramagnetic phase at zero magnetic field, $M_{i}^{(\alpha)}=$ 0 for all $\alpha$. In an ordered state $M_{i}^{(\alpha)} \neq 0$.

Exact equations for magnetic moments of the Potts model on a treelike complex network (see Appendix E) were derived by Dorogovtsev et al. (2004) by using the recursion method which, as we have demonstrated, is equivalent to the Bethe-Peierls approximation and the belief-propagation algorithm . It was shown that the ferromagnetic $p$-state Potts model with couplings $\left(J_{i j}=\right.$ $J>0)$ on the configuration model has the critical temperature

$$
T_{\mathrm{P}}=J / \ln \left[\frac{B+p-1}{B-1}\right] .
$$

where $B=z_{2} / z_{1}$ is the average branching parameter. Interestingly, $T_{\mathrm{P}}$ has different meanings for $p=1,2$ and $p \geqslant 3$. In the case $p=1$, the critical temperature $T_{\mathrm{P}}$ determines the percolation threshold (see Appendix E). When $p=2, T_{\mathrm{P}}$ is equal to the exact critical temperature Eq. (84) of the ferromagnetic phase transition in the Ising model (it is only necessary to rescale $J \rightarrow 2 J$ ). For $p \geqslant 3, T_{\mathrm{P}}$ gives the lower temperature boundary of the hysteresis phenomenon at the first order phase transition.

\section{B. A first order transition}

In the standard mean-field theory, the ferromagnetic Potts model with $J_{i j}=J>0$ undergoes a first order phase transition for all $p \geqslant 3(\mathrm{Wu}, 1982)$. In order to study critical properties of the Potts model on a complex network, we need to solve Eq. (E2) which is very difficult to do analytically. An approximate solution based on the ansatz Eq. (85) was obtained by Dorogovtsev et al. (2004). It turned out that in uncorrelated random networks with a finite second moment $\left\langle q^{2}\right\rangle$ (which corresponds to scale-free networks with $\gamma>3$ ) a first order phase transition occurs at a critical temperature $T_{c}$ if the number of Potts states $p \geqslant 3$. In the region $T_{\mathrm{P}}<T<T_{c}$, two metastable thermodynamic states with magnetic moments $M=0$ and $M \neq 0$ coexist. This leads to hysteresis

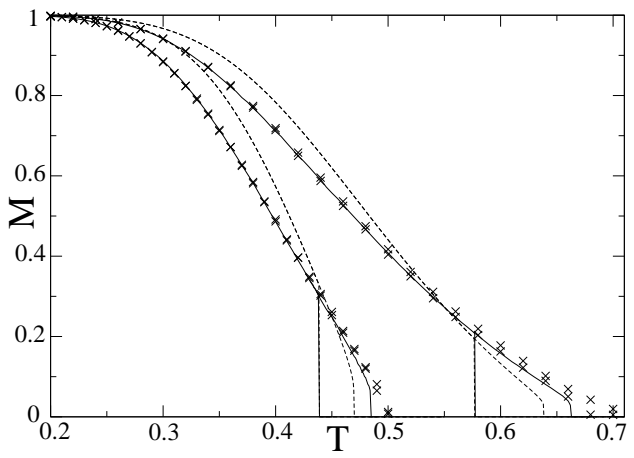

FIG. 33 Magnetic moment $M$ versus $T$ for the ferromagnetic Potts model on an uncorrelated scale-free network with degree $z_{1}=10$. Leftmost curves: $\gamma=4$; rightmost curves, $\gamma=3.5$. Numerical simulations and exact numerical solution (Ehrhardt and Marsili, 2005) are shown in crosses and solid lines. Dotted lines, an approximate solution (Dorogovtsev et al., 2004). Vertical lines, the lower temperature boundary $T_{\mathrm{P}}$ of the hysteresis region. From Ehrhardt and Marsili (2005).

phenomena which are typical for a first order phase transition. At $T<T_{\mathrm{P}}$, only the ordered state with $M \neq 0$ is stable.

When $\gamma$ tends to 3 from above, $T_{c}$ increases while the jump of the magnetic moment at the first order phase transition tends to zero. The influence of the network heterogeneity becomes dramatic when $2<\gamma \leqslant 3$ and the second moment $\left\langle q^{2}\right\rangle$ diverges: instead of a first order phase transition, the $p$-state Potts model with $p \geqslant 3$ undergoes an infinite order phase transition at the critical temperature $T_{c}(N) / J \approx z_{2} /\left(z_{1} p\right) \gg 1$, similarly to the Ising model in Sec. VI.C.2 In the limit $N \rightarrow \infty$, the Potts model is ordered at any finite $T$.

Ehrhardt and Marsili (2005) used a population dynamics algorithm to solve numerically Eq. (E2) for uncorrelated scale-free networks. The exact numerical calculations and numerical simulations of the Potts model confirmed that a first order phase transition occurs at $p \geqslant 3$ when $\gamma>3$. Some results obtained by Ehrhardt and Marsili (2005) are represented in Fig. 33. where they are compared with the approximate solution. As one could expected, the approximate solution gives poor results for vertices with small degree. For graphs with a large minimum degree (say $q_{0}=10$ ) the approximate solution agrees well with the exact calculations and numerical simulations.

A simple mean-field approach to the Potts model on uncorrelated scale-free networks was used by Iglói and Turban (2002). Its conclusions essentially deviate from the exact results. Karsai et al. (2007) studied the ferromagnetic large- $p$ state Potts model on evolving networks and described finite-size scaling in these systems. 


\section{Coloring a graph}

Coloring random graphs is a remarkable problem in combinatorics (Garey and Johnson, 1979) and statistical physics (Wu, 1982). Given a graph, we want to know if this graph can be colored with $p$ colors in such a way that no two neighboring vertices have the same color. A famous theorem states that four colors is sufficient to color a planar graph, such as a political map (Appel and Haken, 1977a,b). Coloring a graph is not only beautiful mathematics but it also has important applications. Good examples are scheduling of registers in the central processing unit of computers, frequency assignment in mobile radios, and pattern matching. Coloring a graph is a NP complete problem. The time needed to properly color a graph grows exponentially with the graph size.

How many colors do we need to color a graph? Intuitively it is clear that any graph can be colored if we have a large enough number of colors, $p$. The minimum needed number of colors is called the "chromatic number" of the graph. The chromatic number is determined by the graph structure. It is also interesting to find the number of ways one can color a graph.

The coloring problem was extensively investigated for classical random graphs. There exists a critical degree $c_{p}$ above which the graph becomes uncolorable by $p$ colors with high probability. This transition is the so called $p$-COL/UNCOL transition. Only graphs with average degree $z_{1} \equiv\langle q\rangle<c_{p}$ may be colored with $p$ colors. For larger $z_{1}$ we need more colors.

In order to estimate the threshold degree $c_{p}$ for the Erdős-Rényi graph, one can use the so-called firstmoment method (annealed computation, in other words). Suppose that $p$ colors are assigned randomly to vertices. It means that a vertex may have any color with equal probability $1 / p$. The probability that two ends of a randomly chosen edge have different colors is $1-1 / p$. We can color $N$ vertices of the graph in $p^{N}$ different ways. However only a small fraction $(1-1 / p)^{L}$ of these configurations have the property that all $L=z_{1} N / 2$ edges connect vertices of different colors. Hence the number of $p$-colorable configurations is

$$
\mathfrak{N}\left(z_{1}\right)=p^{N}(1-1 / p)^{L} \equiv \exp [N \Xi(p)]
$$

If $\Xi(p) \geqslant 0$, then with high probability there is at least one $p$-colorable configuration. At $p \gg 1$, this condition leads to the threshold average degree $c_{p} \sim 2 p \ln p-\ln p$. The exact threshold $c_{p} \sim 2 p \ln p-\ln p+o(1)$ was found by Luczak (1991), see also Achlioptas et al. (2005).

The coloring problem was reconsidered by methods of statistical mechanics of disordered systems, and a complex structure of the colorable phase was revealed (Braunsteinet al., 2003b; Krzakała et al., 2004; Mézard et al., 2005; Mulet et al., 2002). It was found that the colorable phase itself contains several different phases. These studies used the equivalence of this problem to the problem of finding the ground state of the
Potts model, Eq. (119), with $p$ states (colors) and antiferromagnetic interactions $J_{i j}=-J<0$ in zero field. Within this approach, the graph is $p$-colorable if in the ground state the endpoints of all edges are in different Potts states. The corresponding ground state energy is $E=0$. The degeneracy of this ground state means that there are several ways for coloring a graph. In the case of a $p$-uncolorable graph, the ground state energy of the antiferromagnetic Potts model is positive due to a positive contribution from pairs of neighboring vertices having the same color.

It was shown that if the mean degree $z_{1}$ is sufficiently small, then it is easy to find a solution of the problem by using usual computational algorithms. In these algorithms, colors of one or several randomly chosen vertices are changed one by one. For example, the Metropolis algorithm gives an exponentially fast relaxation from an arbitrary initial set of vertex colors to a correct solution (Svenson and Nordahl, 1999). On the other hand, for higher mean degrees (of course, still below $c_{p}$ ), these algorithms can approach a solution only in non-polynomial times- "computational hardness". The computational hardness is related to the presence of a hierarchy of numerous "metastable" states with a positive energy, which can dramatically slow down or even trap any simple numerical algorithm.

The mentioned works focused on the structure of the space of solutions for coloring a graph. (A solution here is a proper coloring of a graph.) It was found that this structure qualitatively varies with the mean degree. In general, the space of solutions is organized as a set of disjoint clusters - "pure states". Each of these clusters consists of solutions which can be approached from each other by changing colors of only $o(N)$ vertices. On the other hand, to transform a solution belonging to one cluster into a solution in another cluster, we have to change colors of $O(N)$ vertices, i.e., of a finite fraction of vertices. Clearly, if a network consists of only bare vertices $\left(z_{1}=0\right)$, the space of solutions consists of a single cluster. However, above some threshold value of a mean degree, this space becomes highly clustered. The structure and statistics of these clusters at a given $z_{1}$ determine whether the coloring problem is computationally hard or not.

The statistics of clusters in a full range of mean degrees was obtained in Krzakała et al. (2007) and Zdeborová and Krzakała (2007). Their results indicate a chain of topologically different phases inside the colorable phase, see Fig. 34. An important notion in this kind of problems is a frozen variable. (A variable here is a vertex.) By definition, a frozen variable (vertex) has the same color in all solutions of a given cluster. Figure 34 demonstrates that the clusters with frozen variables are statistically dominating in the range $c_{r}<z_{1}<c_{p}$. Remarkably, the computational hardness was observed only in this region, although the replica symmetry breaking was found in the essentially wider range $c_{d}<z_{1}<c_{p}$.

Coloring the Watts-Strogatz small-world networks was 


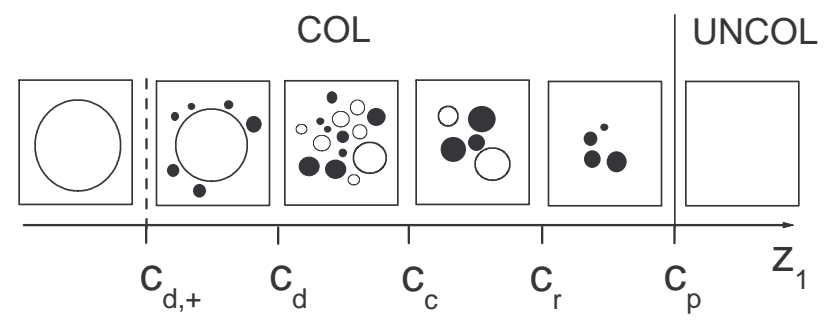

FIG. 34 Schematic phase diagram and structure of solutions for coloring the Erdös-Rényi graphs in $p$-colors versus the average degree $z_{1}$. (i) $z_{1}<c_{d,+}$, solutions form one large connected cluster without frozen variables (open circle). (ii) $c_{d,+}<z_{1}<c_{d}$, in addition to a large cluster, small disjoint clusters with frozen variables (black circles) appear. They include however an exponentially small fraction of solutions. (iii) $c_{d}<z_{1}<c_{c}$, solutions are arranged in exponentially many clusters of different sizes with and without frozen variables. Exponentially many clusters without frozen variables dominate. (iv) $c_{c}<z_{1}<c_{r}$, there are a finite number statistically dominating large clusters. These clusters does not contain frozen variables. (v) $c_{r}<z_{1}<c_{p}$, dominating clusters contain frozen variables. Above $c_{p}$, a graph is $p$-uncolorable. $c_{d,+}$ coincides with the 2 -core birth point. $c_{d}$, $c_{c}$, and $c_{r}$ correspond to so-called clustering, condensation, and rigidity (freezing) transitions, respectively. Adapted from Zdeborová and Krzakała (2007).

studied numerically by Walsh (1999). He found that it is easy to color these networks at small and large densities of shortcuts, $p$. However it is hard to color them in the intermediate region of $p$.

\section{Extracting communities}

It is a matter of common experience that a complex system or a data set may consist of clusters, communities or groups. A common property of a network having a community structure is that edges are arranged denser within a community and sparser between communities. If a system is small, we can reveal a community structure by eye. For a large network we need a special method (Newman, 2003a). Statistical physics can provide useful tools for this purpose. In particular, the Potts model has interesting applications which are ranged from extracting species of flowers, collective listening habits, communities in a football league to a search of groups of configurations in a protein folding network.

Blatt et al. (1996) showed that a search for clusters in a data set can be mapped to extracting superparamagnetic clusters in the ferromagnetic Potts model formed in the following way: each point in the data set is represented as a point in a $d$-dimensional space. The dimensionality $d$ is determined by the number of parameters we use to describe a data point, e.g., color, shape, size etc. A Potts spin is assigned to each of these points. The strength of short-range ferromagnetic interaction between nearest neighboring spins is calculated following a certain rule: the larger the distance between two neighboring points in the space the smaller is the strength. The energy of the model is given by Eq. (119). Blatt et al. (1996) used Monte Carlo simulations of the Potts model at several temperatures in order to reveal clusters of spins with strong ferromagnetic correlations between neighboring spins. Clusters of aligned spins showed a superparamagnetic behavior at low temperatures. They were identified as clusters in the data set. An analysis of real data, such as Iris data and data taken from a satellite image of the Earth, demonstrated a good performance of the method.

Lambiotte and Ausloos (2005) studied a complex bipartite network formed by musical groups and listeners. Their aim was to uncover collective listening habits. These authors represented individual musical signatures of people as Potts vectors. A scalar product of the vectors characterized a correlation between music tastes. This investigation found that collective listening habits do not fit the usual genres defined by the music industry.

Reichardt and Bornholdt (2004, 2006) proposed to map the communities of a network onto the magnetic domains forming the ground state of the $p$-state Potts model. In this approach, each vertex in the network is assigned a Potts state $\alpha=1,2, \ldots p$. Vertices, which are in the same Potts state $\alpha$, belong to the same community $\alpha$. The authors used the following Hamiltonian:

$$
\mathcal{H}=-\frac{1}{2} \sum_{i, j} a_{i j} \delta_{\alpha_{i}, \alpha_{j}}+\frac{\lambda}{2} \sum_{\alpha=1}^{p} n_{s}\left(n_{s}-1\right),
$$

where $a_{i j}$ are the elements of the adjacency matrix of the network, $n_{s}$ is the number of vertices in the community $\alpha$, i.e., $n_{s}=\sum_{i} \delta_{\alpha_{i}, \alpha}$. The number of possible states, $p$, is chosen large enough to take into account all possible communities. $\lambda$ is a tunable parameter. The first sum in Eq. (123) is the energy of the ferromagnetic Potts model. It favors merging vertices into one community. The second repulsive term is minimal when the network is partitioned into as many communities as possible. In this approach the communities arise as domains of aligned Potts spins in the ground state which can be found by Monte Carlo optimization.

At $\lambda=1$ the energy Eq. (123) is proportional to the modularity measure $Q$, namely $\mathcal{H}=-Q L$ where $L$ is the total number of edges in the network. Thus the ground state of the model Eq. (123) corresponds to the maximum modularity $Q$. The modularity measure was introduced in Clauset et al. (2004); Newman and Girvan (2004). For a given partition of a network into communities, the modularity is the difference between the fraction of edges within communities and the expected fraction of such edges under an appropriate null model of the network (a random network model assuming the absence of a modular structure):

$$
Q=\sum_{\alpha}\left(\frac{L_{\alpha}}{L}-\frac{L_{\alpha}^{\exp }}{L}\right)=\frac{1}{2 L} \sum_{\alpha} \sum_{i, j}\left(a_{i j}-p_{i j}\right) \delta_{\alpha_{i}, \alpha_{j}} .
$$


Here $L_{\alpha}$ and $L_{\alpha}^{\exp }$ are the numbers of edges within community $\alpha$ in the network and in its null model, respectively; $p_{i j}$ is the probability that vertices $i$ and $j$ are connected in the null model. Reichardt and Bornholdt (2004, 2006) used the configuration model as the null model, i.e., $p_{i j}=q_{i} q_{j} / 2 L$ where $q_{i}$ and $q_{j}$ are degrees of vertices $i$ and $j$ respectively. Tuning $\lambda$ and $p$, one can find a partition of a given network into communities such that a density of edges inside communities is maximal when compared to one in a completely random network. If however the size distribution of communities is sufficiently broad, then it is not easy to find an optimal value of the parameter $\lambda$. Searching for small communities and the resolution limit of this method are discussed in Kumpula et al. (2007). Interestingly, finding the partition of a complex network into communities, such that it maximizes the modularity measure, is an NP-complete problem (Brandes et al., 2006).

Reichardt and Bornholdt (2004, 2006) applied the Potts model Eq. (123) to study a community structure of real world networks, such as a US college football network and a large protein folding network.

Guimerà et al. (2004) proposed another approach to the problem of extracting communities based on a specific relation between the modularity measure $Q$ and the ground state energy of a Potts model with multiple interactions.

\section{THE XY MODEL ON NETWORKS}

The $X Y$ model describes interacting planar rotators. The energy of the $X Y$ model on a graph is

$$
\mathcal{H}=-\frac{J}{2} \sum_{i, j} a_{i j} \cos \left(\theta_{i}-\theta_{j}\right)
$$

where $a_{i j}$ are the elements of the adjacency matrix of the graph, $\theta_{i}$ is the phase of a rotator at vertex $i, J$ is the coupling strength. Unlike the Ising and Potts models with discrete spins, the $X Y$ model is described by continuous local parameters and belongs to the class of models with continuous symmetry.

A one-dimensional $X Y$ model has no phase transition. On a two-dimensional regular lattice, this model $(J>0)$ undergoes the unusual Berezinskii-KosterlitzThouless phase transition. On a $d$-dimensional lattice at $d>4$ and the fully connected graph (Antoni and Ruffo, 1995) the ferromagnetic phase transition in the $X Y$ model is of second order with the standard mean-field critical exponents.

The study of the $X Y$ model on complex networks is motivated by several reasons. In principal, the continuous symmetry may lead to a new type of critical behavior in complex networks. Moreover the ferromagnetic $X Y$ model is close to the Kuramoto model which is used for describing the synchronization phenomenon in Sec. X.A.

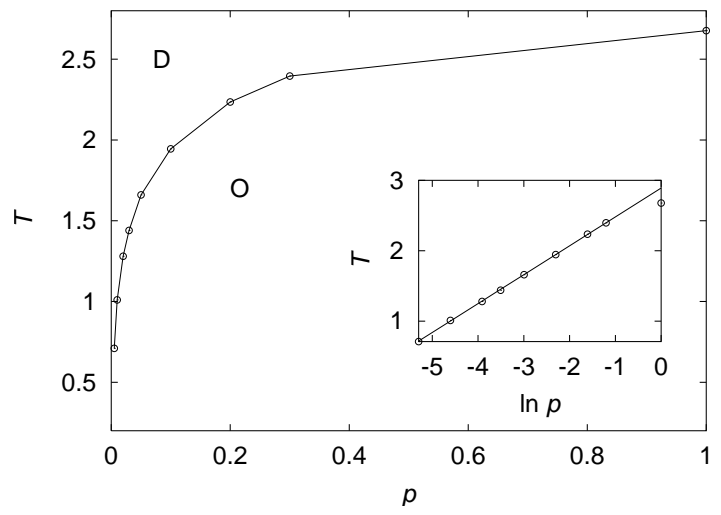

FIG. $35 p-T$ phase diagram of the ferromagnetic $X Y$ model on the Watts-Strogatz network. $p$ is the fraction of shortcuts. $D$ and $O$ denote the disordered and ordered phases, respectively. The inset shows that the critical temperature is well approximated by a function $0.41 \ln p+2.89$. From Kim et al. (2001).

\section{A. The $X Y$ model on small-world networks}

There were a few studies of the $X Y$ model on complex networks. Kim et al. (2001) carried out MonteCarlo simulations of the ferromagnetic $X Y$ model on the Watts-Strogatz small-world network generated from a ring of $N$ vertices. They measured the order parameter $r=\left|N^{-1} \sum_{j} \exp \left(i \theta_{j}\right)\right|$. By using the standard finite-size scaling analysis they showed that the phase transition appears even at a tiny fraction of shortcuts, $p$. The transition is of second order with the standard mean-field critical exponent $\beta=1 / 2$ (similar to the phase transition in the Ising model in Sec. (VI.D). The phase diagram of the $X Y$ model is shown in Fig. 35. There is no phase transition at $p=0$ because the system is one-dimensional. Surprisingly, the dependence of the critical temperature $T_{c}$ on $p$ was well fitted by a function $T_{c}(p) / J=0.41 \ln p+2.89$ in contrast to $T_{c}(p) / J \propto 1 /|\ln p|$ for the Ising model. The origin of this difference is unclear. Dynamical Monte-Carlo simulations of Medvedyeva et al. (2003) confirmed the meanfield nature of the phase transition. These authors found that, at $T$ near $T_{c}$, the characteristic time $\tau$ scales as $\tau \sim \sqrt{N}$ as it should be for networks with rapidly decreasing degree distribution (see the theory of finite-size scaling in Sec. IX.B.

\section{B. The $X Y$ model on uncorrelated networks}

An exact solution of the $X Y$ model on tree-like networks, in principle, can obtained in the framework of the belief propagation algorithm, see Eq. (69). Another analytical approach based on the replica theory and the cavity method was developed by Coolen et al. (2005); Skantzos et al. (2005), see also Skantzos and Hatchett 
(2007) for the dynamics of a related model. We here consider the influence of network topology on the critical behavior of the $X Y$ model, using the annealed network approximation from Sec. VI.A.3. Recall that in this approach, the element of adjacency matrix $a_{i j}$ is replaced by the probability that two vertices $i$ and $j$ with degrees $q_{i}$ and $q_{j}$, are linked. In this way, for the configuration model, we obtain the $X Y$ model with a degree dependent coupling on the fully connected graph:

$$
\mathcal{H}_{\mathrm{MF}}=-\frac{J}{N z_{1}} \sum_{i<j} q_{i} q_{j} \cos \left(\theta_{i}-\theta_{j}\right)
$$

This model is solved exactly by using a weighted complex order parameter

$$
\widetilde{r} e^{i \psi}=\frac{1}{N z_{1}} \sum_{j=1}^{N} q_{j} e^{i \theta_{j}} .
$$

The phase $\psi$ determines the direction along which rotators are spontaneously aligned. The order parameter $\widetilde{r}$ is a solution of an equation:

$$
\widetilde{r}=\frac{1}{z_{1}} \sum_{q} P(q) q \frac{I_{1}(\widetilde{r} q \beta J)}{I_{0}(\widetilde{r} q \beta J)},
$$

where $I_{0}(x)$ and $I_{1}(x)$ are the modified Bessel functions. An analysis of Eq. (128) shows that the ferromagnetic $X Y$ model has the same critical behavior as the ferromagnetic Ising model. Notice that the usually used order parameter $r=\left|N^{-1} \sum_{j} e^{i \theta_{j}}\right|$ has the same critical behavior as $\widetilde{r}$. The critical temperature of the continuous phase transition is $T_{c}=J\left\langle q^{2}\right\rangle / 2 z_{1}$. It is finite in a complex network with a finite second moment $\left\langle q^{2}\right\rangle$, and diverges if $\left\langle q^{2}\right\rangle \rightarrow \infty$. In the latter case, the $X Y$ model is in the ordered state at any finite $T$. The annealed network approximation predicts that the $X Y$ model on the WattsStrogatz small-world network has the standard meanfield critical behavior. This agrees with the numerical simulations in Kim et al. (2001) and Medvedveva et al. (2003).

\section{PHENOMENOLOGY OF CRITICAL PHENOMENA IN NETWORKS}

Why do critical phenomena in networks differ so strongly from those in usual substrates and what is their common origin? Why do all investigated models demonstrate universal behavior when $\left\langle q^{2}\right\rangle$ diverges? In order to answer these questions and analyze results of simulations and experiments from a general point of view, we need a general theory which is not restricted by specific properties of any model.

In the phenomenological approach, the origin of interactions and nature of interacting objects are irrelevant. In this section, we consider a phenomenological theory of cooperative phenomena in networks proposed by Goltsev et al. (2003). This theory is based on concepts of the Landau theory of continuous phase transitions and leads to the conclusion that the universal critical behavior in networks is determined by two factors: (i) the structure of a network and (ii) the symmetry of a given model.

\section{A. Generalized Landau theory}

Let us consider a system of interacting objects. Interactions or links between these objects form a net. We assume that some kind of order can emerge. This ordered phase may be characterized by some quantitative characteristic $x$ while it will vanish in a disordered phase above a critical point. We assume that the thermodynamic potential $\Phi$ of the system is not only a function of the order parameter $x$ but also depends on the degree distribution:

$$
\Phi(x, H)=-H x+\sum_{q}^{\infty} P(q) \phi(x, q x) .
$$

Here $H$ is a field conjugated with $x$. Equation (129) is not obvious a priori. The function $\phi(x, q x)$ can be considered as a contribution of vertices with $q$ connections. There are arguments in favor of this assumption. Let us consider the interaction of an arbitrary vertex with $q$ neighboring vertices. In the framework of a mean-field approach, $q$ neighbors with a spontaneous "moment" $x$ produce an effective field $q x$ acting on this vertex.

We impose only general restrictions on $\phi(x, y)$ :

(i) $\phi(x, y)$ is a smooth function of $x$ and $y$ and can be represented as a series in powers of both $x$ and $y$. Coefficients of this series are functions of "temperature" $T$ and "field" $H$.

(ii) $\Phi(x, H)$ is finite for any finite average degree $\langle q\rangle$.

A network topology affects analytical properties of $\Phi$. If the distribution function $P(q)$ has a divergent moment $\left\langle q^{p}\right\rangle$, then we have

$$
\Phi(x, H)=-H x+\sum_{n=2}^{p-1} f_{n} x^{n}+x^{p} s(x),
$$

where $s(x)$ is a non-analytic function. The specific form of $s(x)$ is determined by the asymptotic behavior of $P(q)$ at $q \gg 1$. It is the nonanalytic term that can lead to a deviation from the standard mean-field behavior.

Following Landau, we assume that near the critical temperature the coefficient $f_{2}$ can be written as $a(T-$ $T_{c}$ ) where $a$ is chosen to be positive for the stability of the disordered phase. The stability of the ordered phase demands that either $f_{3}>0$ or if $f_{3}=0$, then $f_{4}>0$. The order parameter $x$ is determined by the condition that $\Phi(x, H)$ is minimum: $d \Phi(x, H) / d x=0$. 
If degree distribution exponent $\gamma$ is non-integer, then the leading nonanalytic term in $\Phi(x)$ is $x^{\gamma-1}$. If $\gamma$ is integer, then the leading nonanalytic term is $x^{\gamma-1}|\ln x|$. Interestingly, this nonanaliticity looks like that of the free energy for the ferromagnetic Ising model in magnetic field on a Cayley tree (see Sec. VI.B).

We must be take into account a symmetry of the system. When $\Phi(x, H)=\Phi(-x,-H)$ and the coefficient $f_{4}$ is positive, we arrive at the critical behavior which describes the ferromagnetic Ising model on equilibrium uncorrelated random networks in Sec. VI.C.2, In a network with $\left\langle q^{4}\right\rangle<\infty$ a singular term in $\Phi$ is irrelevant, and we have the usual $x^{4}$ - Landau theory which leads to the standard mean-field phase transition. The singular term $x^{\gamma-1}$ becomes relevant for $2<\gamma \leq 5$ (this term is $x^{4}|\ln x|$ at $\gamma=5$ ). Critical exponents are given in Table I. At the critical point $T=T_{c}$ the order parameter $x$ is a non-analytic function of $H: x \propto H^{1 / \delta}$, where $\delta(\gamma>5)=3$ and $\delta(3<\gamma<5)=\gamma-2$.

If the symmetry of the system permits odd powers of $x$ in $\Phi$ and $f_{3}$ is positive, then the phenomenological approach gives a critical behavior which was found for percolation on uncorrelated random networks in Sec. III.B.2. Note that when $\gamma>4$, a singular term $x^{\gamma-1}$ is irrelevant. It becomes relevant for $2<\gamma \leq 4$ (this term is $x^{3}|\ln x|$ at $\left.\gamma=4\right)$.

At $2<\gamma \leqslant 3$, the thermodynamic potential has a universal form, independent on the symmetry:

$$
\Phi(x, H)=-H x+C x^{2}-D s(x),
$$

where $s(x)=x^{2}|\ln x|$ for $\gamma=3$, and $s(x)=x^{\gamma-1}$ for $2<\gamma<3$. We can choose $C \propto T^{2}$ and $D \propto T$, then the phenomenological theory gives a correct temperature behavior of the ferromagnetic Ising model.

When $f_{3}<0$ (or $f_{4}<0$ if $f_{3}=0$ ), the phenomenological theory predicts a first-order phase transition for a finite $\left\langle q^{2}\right\rangle$. This corresponds, e.g., to the ferromagnetic Potts model with $p \geq 3$ states (see Sec. VII).

The phenomenological approach agrees with the microscopic theory and numerical simulations of the ferromagnetic Ising, Potts, $X Y$, spin glass, Kuramoto and the random-field Ising models, percolation and epidemic spreading on various uncorrelated random networks. These models have also been studied on complex networks with different clustering coefficients, degree correlations, etc. It seems that these characteristics are not relevant, or at least not essentially relevant, to critical behavior. When the tree ansatz for complex networks gives exact results, the phenomenology leads to the same conclusions. In these situations the critical fluctuations are Gaussian. We strongly suggest that the critical fluctuations are Gaussian in all networks with the small-world effect, as is natural for infinite-dimensional objects.

\section{B. Finite-size scaling}

Based on the phenomenological theory one can get scaling exponents for finite-size scaling phenomena in complex networks. Let $\Phi(m, \tau, H, N)$ be a thermodynamic potential per vertex, where $\tau$ is the deviation from a critical point. According to the standard scaling hypothesis (in its finite-size scaling form), in the critical region,

$$
N \Phi(m, \tau, H, N)=f_{\phi}\left(m N^{x}, \tau N^{y}, H N^{z}\right),
$$

where $f_{\phi}(a, b, c)$ is a scaling function. Note that there is exactly $N$ on the left-hand side of this relation and not an arbitrary power of $N$. Formally substituting $\Phi(m, \tau, H)=A \tau m^{2}+B m^{\Delta(\gamma)}-H m$, one can find exponents $x, y$, and $z$. As was explained, $\Delta$ may be (i) $\min (4, \gamma-1)$, as, e.g., in the Ising model, or (ii) $\min (3, \gamma-1)$, as, e.g., in percolation. This naive substitution, however, does not allow one to obtain a proper scaling function, which must be analytical, as is natural. The derivation of the scaling function demands more rigorous calculations.

As a result, for the two classes of theories listed above, we arrive at the following scaling forms of the order parameter:

(i) for $\gamma \geq 5, m(\tau, H, N)=N^{-1 / 4} f\left(N^{1 / 2} \tau, N^{3 / 4} H\right),(133)$

(ii) for $\gamma \geq 4, m(\tau, H, N)=N^{-1 / 3} f\left(N^{1 / 3} \tau, N^{2 / 3} H\right)$, (13

and for more small $3<\gamma<5$ (i) or $3<\gamma<4$ (ii), $m$ scales as

$m(\tau, H, N)=N^{-1 /(\gamma-1)} f\left(N^{(\gamma-3) /(\gamma-1)} \tau, N^{(\gamma-2) /(\gamma-1)} H\right)$.

Hong et al. 2007a) obtained these scaling relations (without field) by using other arguments and confirmed them simulating the Ising model on the static and the configuration models of uncorrelated networks. Their idea may be formally reduced to the following steps. Recall a relevant standard scaling relation from the physics of critical phenomena in lattices. The standard form is usually written for dimension $d$ lower than the upper critical dimension $d_{u}$. So, rewrite this scaling relation for $d>d_{u}$ : substitute $d_{u}$ for $d$ and use the mean-field values of the critical exponents which should be obtained as follows. For networks, in this relation, formally substitute $\nu=1 / 2$ for the correlation length exponent and $\eta=0$ for the Fisher exponent, use the susceptibility exponent $\tilde{\gamma}=\nu(2-\eta)=1$, exponent $\beta=\beta(\gamma)$ (see Sec. IX.A), and

$$
d_{u}(\gamma)=\frac{2 \Delta(\gamma)}{\Delta(\gamma)-2}
$$

(Hong et al., 2007a). This procedure allows one to easily derive various scaling relations. We have used it in Sec. III.B.3,

Finite-size scaling of this kind works in a wide class of models and processes on networks. Hong et al. (2007a) 
also applied these ideas to the contact process on networks. Earlier, Kim et al. (2001) and Medvedyeva et al. (2003) studied the finite size scaling by simulating the $X Y$ model on the Watts-Strogatz network. In their work, in particular, they investigated the dynamic finite-size scaling. In the framework of our phenomenology, we can easily reproduce their results and generalize them to scale-free networks. Let us assume the relaxational dynamics of the order parameter: $\partial m / \partial t=-\partial \Phi(m) / \partial m$. In dynamical models, the scaling hypothesis also implies the scaling time variable, $t_{\mathrm{scal}}=t N^{-s}$, which means that the relaxation time diverges as $N^{s}$ at the critical point. For brevity, we only find the form of this scaling variable, which actually resolves the problem. In terms of scaling variables, the dynamic equation for the order parameter must not contain $N$. With this condition, passing to the scaling variables $m N^{x}$ and $t N^{-s}$ in the dynamic equation, we immediately get $s=y$, which means that time scales with $N$ exactly in the same way as $1 / \tau$. So, for the indicated two classes of theories, (i) and (ii), the time scaling variable is of the following form:

$$
\begin{aligned}
& \text { in theory (i), for } \gamma \geq 5, \quad t_{\text {scal }}=t N^{-1 / 2}, \\
& \text { in theory (ii), for } \gamma \geq 4, \quad t_{\text {scal }}=t N^{-1 / 3},
\end{aligned}
$$

and

in theory (i), for $3<\gamma<5$, and in theory (ii), for $3<\gamma<4$,

$$
t_{\text {scal }}=t N^{-(\gamma-3) /(\gamma-1)} \text {. }
$$

Finally, we recommend that the reader refer to Gallos et al. (2007b) for the finite-size scaling in scalefree networks with fractal properties. For description of these networks, see Song et al. (2005, 2006, 2007) and also Goh et al. (2006).

\section{SYNCHRONIZATION ON NETWORKS}

Emergence of synchronization in a system of coupled individual oscillators is an intriguing phenomenon. Nature gives many well-known examples: synchronously flashing fireflies, crickets that chirp in unison, two pendulum clocks mounted on the same wall synchronize their oscillations, synchronous neural activity, and many others. Different dynamical models were proposed to describe collective synchronization, see, for example, monographs and reviews of Pikovsky et al. (2001), Strogatz (2003), Strogatz (2000), Acebrón et al. (2005), and Boccaletti et al. (2006).

Extensive investigations aimed at searching for network architectures which optimize synchronization. First (mostly numerical) studies of various dynamical models have already revealed that the ability to synchronize can be improved in small-world networks (Barahona and Pecora, 2002; Gade and $\mathrm{Hu}$, 2000; Hong et al., 2002a; Jost and Jov, 2001; Lago-Fernández et al., 2000; Wang and Chen, 2002). On the other hand, an opposite effect was
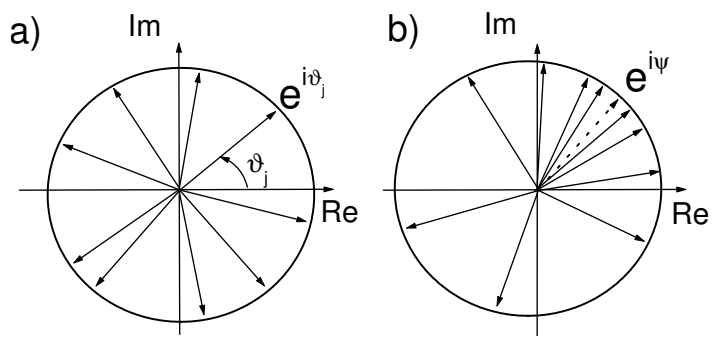

FIG. 36 Schematic view of phases in the Kuramoto model. (a) Incoherent phase. Unit length vectors representing individual states are randomly directed in the complex plane. (b) Coherent phase. The individual states condense around a direction $\psi$.

observed in synchronization dynamics of pulse-coupled oscillators (Guardiola et al., 2000), where homogeneous systems synchronize better.

We here consider the effect of the network topology on the synchronization in the Kuramoto model and a network of coupled dynamical systems. These two models represent two different types of synchronization phenomena. The interested reader will find the discussion of this effect for coupled map lattices in Atav et al. (2004); Gade and $\mathrm{Hu}$ (2000); Grinstein and Linsker (2005); Huang et al. (2006); Jost and Joy (2001); Lind et al. (2004), for networks of Hodgkin-Huxley neurons - in Kwon and Moon (2002); Lago-Fernández et al. (2000), for pulse-coupled oscillators - in Denker et al. (2004); Timme et al. (2004), and for the Edwards-Wilkinson model -in Kozma et al. (2004).

\section{A. The Kuramoto model}

The Kuramoto model is a classical paradigm for a spontaneous emergence of collective synchronization (Acebrón et al., 2005; Kuramoto, 1984; Strogatz, 2000). It describes collective dynamics of $N$ coupled phase oscillators with phases $\theta_{i}(t), i=1,2, \ldots N$, running at natural frequencies $\omega_{i}$ :

$$
\dot{\theta}_{i}=\omega_{i}+J \sum_{j=1}^{N} a_{i j} \sin \left(\theta_{j}-\theta_{i}\right),
$$

where $a_{i j}$ is the adjacency matrix of a network. $J$ is the coupling strength. The frequencies $\omega_{i}$ are distributed according to a distribution function $g(\omega)$. It is usually assumed that $g(\omega)$ is unimodular and symmetric about its mean frequency $\Omega$. It is convenient to use a rotating frame and redefine $\theta_{i} \rightarrow \theta_{i}-\Omega t$ for all $i$. In this frame we can set the mean of $g(\omega)$ to be zero. The state of oscillator $j$ can be characterized by a complex exponent $\exp \left(i \theta_{j}\right)$ which is represented by a vector of unit length in the complex plane (see Fig. [36).

The Kuramoto model is solved exactly for the fully connected graph (all-to-all interaction), i.e., $a_{i j}=1$ for 
all $i \neq j$, with rescaling $J \rightarrow J / N$. When $J<J_{c}$, there is no collective synchronization between the rotations of individual oscillators. Nonetheless some finite clusters of synchronized oscillators may exist. Collective synchronization between oscillators emerges spontaneously above a critical coupling $J_{c}$ if $N \rightarrow \infty$. The global state of the model is characterized by the following average:

$$
r(t) e^{i \psi(t)}=\frac{1}{N} \sum_{j=1}^{N} e^{i \theta_{j}},
$$

where $r(t)$ is the order parameter which measures the phase coherence, and $\psi(t)$ is the average phase. Simulations show that if we start from any initial state, then at $J<J_{c}$ in the incoherent phase, $r(t)$ decays to a tiny jitter of the order of $O\left(N^{-1 / 2}\right)$. On the other hand, in the coherent phase $\left(J>J_{c}\right)$, the parameter $r(t)$ decays to a finite value $r(t \rightarrow \infty)=r<1$. At $J$ near $J_{c}$, the order parameter $r \propto\left|J-J_{c}\right|^{\beta}$ with $\beta=1 / 2$. In the original frame, $\psi(t)$ rotates uniformly at the mean frequency $\Omega$. Substituting Eq. (140) into Eq. (139) gives

$$
\dot{\theta}_{i}=\omega_{i}+J r \sin \left(\psi-\theta_{i}\right) .
$$

The steady solution of this equation shows that at $J>$ $J_{c}$, a finite fraction of synchronized oscillators emerges. These oscillators rotate coherently at frequency $\Omega$ in the original frame. In the rotating frame, they have individual frequencies $\left|\omega_{i}\right| \leqslant J r$ and their phases are locked according to the equation: $\sin \theta_{i}=\omega_{i} / J r$, where we set $\psi=0$. Others oscillators, having individual frequencies $\left|\omega_{i}\right|>J r$, are "drifting". Their phases are changed nonuniformly in time. The order parameter $r$ satisfies the self-consistent equation:

$$
r=\int_{-J r}^{J r} \sqrt{1-\frac{\omega^{2}}{J^{2} r^{2}}} g(\omega) d \omega,
$$

which gives the critical coupling $J_{c}=2 /[\pi g(0)]$. Note that the order of the synchronization phase transition in the Kuramoto model depends on the distribution $g(\omega)$. In particular, it can be of first order if the natural frequencies are uniformly distributed (Tanaka et al., 1997).

The Kuramoto model on finite networks and lattices shows synchronization if the coupling is sufficiently strong. Is it possible to observe collective synchronization in the Kuramoto model on an infinite regular lattice? For sure, there is no synchronization in a onedimensional system with a short-ranged coupling. According to Hong et al. (2004b, 2005), phase and frequency ordering is absent also in two-dimensional $(d=2)$ lattices; frequency ordering is possible only in three-, four-, and higher-dimensional lattices, while phase ordering is possible only when $d>4$. The value of the upper critical dimension of the Kuramoto model is still under discussion (Acebrón et al., 2005). Simulations in Hong et al. (2007b) indicate the mean-field behavior of the Kuramoto model at $d>4$.

\section{B. Mean-field approach}

The Kuramoto model was recently investigated numerically and analytically on complex networks of different architectures. We here first look at analytical studies and then discuss simulations though the model was first studied numerically in Hong et al. (2002a).

Unfortunately no exact results for the Kuramoto model on complex networks are obtained yet. A finite mean degree and a strong heterogeneity of a complex network make difficult to find an analytical solution of the model. Ichinomiva (2004, 2005) and Lee (2005) developed a simple mean-field theory which is actually equivalent to the annealed network approximation in Sec. VI.A.3, Using this approximation, we arrive at the Kuramoto model with a degree dependent coupling on the fully connected graph:

$$
\dot{\theta}_{i}=\omega_{i}+\frac{J q_{i}}{N z_{1}} \sum_{j=1}^{N} q_{j} \sin \left(\theta_{j}-\theta_{i}\right) .
$$

This effective model can be easily solved exactly. Introducing a weighted order parameter

$$
\widetilde{r}(t) e^{i \widetilde{\psi}(t)}=\frac{1}{N z_{1}} \sum_{j=1}^{N} q_{j} e^{i \theta_{j}},
$$

one can write Eq. (143) as follows:

$$
\dot{\theta}_{i}=\omega_{i}+J \widetilde{r} q_{i} \sin \left(\widetilde{\psi}-\theta_{i}\right) .
$$

The steady solution of this equation shows that in the coherent state, oscillators with individual frequencies $\left|\omega_{i}\right| \leqslant J \widetilde{r} q_{i}$ are synchronized. Their phases are locked and depend on vertex degree: $\sin \theta_{i}=\omega_{i} /\left(J \widetilde{r} q_{i}\right)$, where we set $\widetilde{\psi}=0$. This result shows that hubs with degree $q_{i} \gg 1$ synchronize more easy than oscillators with low degrees. The larger the degree $q_{i}$ the larger the probability that an individual frequency $\omega_{i}$ of an oscillator $i$ falls into the range $\left[-J \widetilde{r} q_{i}, J \widetilde{r} q_{i}\right]$. Other oscillators are drifting. $\widetilde{r}$ is a solution of the equation:

$$
\widetilde{r}=\sum_{q} \frac{P(q) q}{z_{1}} \int_{-J \widetilde{r} q}^{J \widetilde{r} q} \sqrt{1-\frac{\omega^{2}}{(J \widetilde{r} q)^{2}}} g(\omega) d \omega .
$$

Spontaneous synchronization with $\widetilde{r}>0$ emerges above the critical coupling

$$
J_{c}=\frac{2 z_{1}}{\pi g(0)\left\langle q^{2}\right\rangle}
$$

which strongly depends on the degree distribution. $J_{c}$ is finite if the second moment $\left\langle q^{2}\right\rangle$ is finite. Note that at a fixed mean degree $z_{1}, J_{c}$ decreases (i.e., the network synchronizes easily) with increasing $\left\langle q^{2}\right\rangle$-increasing heterogeneity. Similarly to percolation, if the moment $\left\langle q^{2}\right\rangle$ diverges (i.e., $2<\gamma \leq 3$ ), the synchronization threshold 
$J_{c}$ is absent, and the synchronization is robust against random failures. In finite networks, the critical coupling is finite, $J_{c}(N) \propto 1 / q_{\text {cut }}^{3-\gamma}(N)$, and is determined by the size-dependent cutoff $q_{\text {cut }}(N)$ in Sec. I.E.4

Another important result, which follows from Eq. (146), is that the network topology strongly influences the critical behavior of the order parameter $\widetilde{r}$. Lee (2005) found that the critical singularity of this parameter is described by the standard mean-field critical exponent $\beta=1 / 2$ if an uncorrelated network has a finite fourth moment $\left\langle q^{4}\right\rangle$, i.e., $\gamma>5$. If $3<\gamma<5$, then $\beta=1 /(\gamma-3)$. Note that the order parameters $r$, Eq. (140), and $\widetilde{r}$, Eq. (144), have the same critical behavior. Thus, with fixed $z_{1}$, the higher heterogeneity of a network, the better its sinchronizability and the smoother the phase transition. The critical behavior of the Kuramoto model is similar to one found for the ferromagnetic Ising model in Sec. VI.C and confirms the phenomenological theory described in Sec. IX A finitesize scaling analysis of the Kuramoto model in complex networks was carried out by Hong et al. (2007c). Within the mean-field theory, they found that the order parameter $\widetilde{r}$ has the finite-size scaling behavior,

$$
\widetilde{r}=N^{-\beta \bar{\nu}} f\left(\left(J-J_{c}\right) N^{1 / \bar{\nu}}\right),
$$

with the critical exponent $\beta$ found above. Remarkably, the critical exponent $\bar{\nu}$ is different from that of the Ising model in Sec. IX.B. namely, $\bar{\nu}=5 / 2$ at $\gamma>5, \bar{\nu}=$ $(2 \gamma-5) /(\gamma-3)$ at $4<\gamma<5, \bar{\nu}=(\gamma-1) /(\gamma-3)$ at $3<\gamma<4$. Simulations of the Kuramoto model carried out by Hong et al. (2007d) agree with these analytical results.

The mean-field theory of synchronization is based on the assumption that every oscillator "feels" a "mean field" created by nearest neighbors. This assumption is valid if the average degree $z_{1}$ is sufficiently large. In order to improve the mean-field theory, Restrepo et al. (2005) introduced a local order parameter at vertex $n$,

$$
r_{n} e^{i \psi_{n}}=\sum_{m} a_{n m} e^{i \theta_{m}},
$$

and found it by using intuitive arguments. In their approach the critical coupling $J_{c}$ is inversely proportional to the maximum eigenvalue $\lambda_{\max }$ of the adjacency matrix $a_{i j}$. However, in an uncorrelated random complex network, the maximum eigenvalue $\lambda_{\max }$ is determined by the cutoff $q_{c u t}(N)$ of the degree distribution, $\lambda_{\max } \approx q_{\text {rut }}^{1 / 2}(N)$ (Chung et al., 2003; Dorogovtsev et al. , 2003; Krivelevich and Sudakov, 2003). In scale-free networks $(\gamma<\infty)$, the cutoff diverges in the limit $N \rightarrow \infty$. Therefore this approach predicts $J_{c}=0$ in the thermodynamic limit even for a scale-free network with $\gamma>3$ in sharp contrast to the approach of Ichinomiva (2004) and Lee (2005).

Oh et al. (2007) studied the Kuramoto model with asymmetric degree dependent coupling $J q_{i}^{-\eta} a_{i j}$ instead of $J a_{i j}$ in Eq. (139) by using the mean-field theory. They found that tuning the exponent $\eta$ changes the critical behavior of collective synchronization. On scale-free networks, this model has a rich phase diagram in the plane $(\eta, \gamma)$. In the case $\eta=1$, the critical coupling $J_{c}$ is finite even in a scale-free network with $2<\gamma<3$ contrary to $J_{c}=0$ for the symmetric coupling which corresponds to $\eta=0$. Note that the influence of the degree dependent coupling is similar to the effect of degree dependent interactions on the phase transition in the ferromagnetic Ising model (see Sec. VI.C.5).

\section{Numerical study of the Kuramoto model}

The Kuramoto model was investigated numerically on various networks. Hong et al. (2002a) studied numerically the model on the Watts-Strogatz network generated from a one-dimensional regular lattice. They observed that collective synchronization emerges even for a tiny fraction of shortcuts, $p$, which make the one-dimensional lattice to be a small world. The critical coupling $J_{c}$ is well approximated as follows: $J_{c}(p) \approx 2 /[\pi g(0)]+a p^{-1}$, where $a$ is a constant. As one might expect, the synchronization phase transition is of second order with the standard critical exponent $\beta=0.5$.

The evolution of synchronization in the Kuramoto model on the Erdős-Rényi and scale-free networks was recently studied by Gómez-Gardeñes et al. (2007a,b). These authors solved numerically Eq. (139) for $N=1000$ coupled phase oscillators and demonstrated that (i) the synchronization on a scale-free network $(\gamma=3)$ appears at a smaller critical coupling $J_{c}$ than the one on the Erdös-Rényi network (with the same average degree as the scale-free network), (ii) the synchronization phase transition on the Erdös-Rényi network is sharper than the transition on the scale-free network. This critical behavior agrees qualitatively with the mean-field theory. Gómez-Gardeñes et al. (2007a,b) calculated a fraction of synchronized pairs of neighboring oscillators for several values of the coupling $J$ and revealed an interesting difference in the synchronization patterns between the Erdős-Rényi and scale-free networks (see Fig. 37). In a scale-free network, a central core of synchronized oscillators formed by hubs grows with $J$ by absorbing small synchronized clusters. In contrast, in the Erdős-Rényi network numerous small synchronized clusters homogeneously spread over the graph. As $J$ approaches $J_{c}$, they progressively merge together and form larger clusters.

Moreno and Pacheco (2004) carried out numerical study of the Kuramoto model on the Barabási-Albert network of size $N=5 \times 10^{4}$. They found that the critical coupling is finite, though small. Surprisingly, the measured critical exponent was close to the standard meanfield value, $\beta \sim 0.5$, contrary to an infinite order phase transition and zero $J_{c}$ predicted by the mean-field theory in the limit $N \rightarrow \infty$. A reason of this discrepancy is unclear.

A community (modular) structure of complex networks 


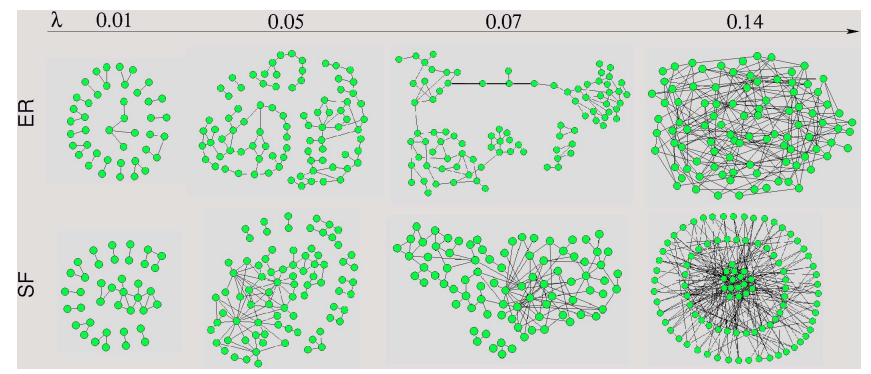

FIG. 37 Synchronization patterns of Erdős-Rényi (ER) and scale-free (SF) networks for several values of coupling $\lambda$ (or $J$ in our notations). From Gómez-Gardeñes et al. (2007a).

makes a strong effect on synchronization. In such networks, oscillators inside a community are synchronized first because edges within a community are arranged denser than edges between communities. On the other hand, intercommunity edges stimulate the global synchronization. The role of network motifs for the synchronization in the Kuramoto model was first studied numerically by Moreno et al. (2004). Oh et al. (2005) solved numerically the dynamical equations Eq. (139) with the asymmetric degree dependent coupling $J q_{i}^{-1} a_{i j}$ for two real networks - the yeast protein interaction network and the Internet at the Autonomous system level. These networks have different community structures. In the yeast protein network, communities are connected diversely while in the Internet communities are connected mainly to the North America continent. It turned out that for a given coupling $J$, the global synchronization for the yeast network is stronger than that for the Internet. These numerical calculations showed that the distributions of phases of oscillators inside communities in the yeast network overlap each other. This corresponds to the mutual synchronization of the communities. In contrast, in the Internet, the phase distributions inside communities do not overlap, the communities are coupled weaker and synchronize independently. A modular structure produces a similar effect on synchronization of coupled-map networks (Huang et al., 2006).

Arenas et al. (2006a, b) showed that the evolution of a synchronization pattern reveals different topological scales at different time scales in a complex network with nested communities. Starting from random initial conditions, highly interconnected clusters of oscillators synchronize first. Then larger and larger communities do the same up to the global coherence. Clustering produces a similar effect. McGraw and Menzinger (2006) studied numerically the synchronization on the Barabasi-Albert networks of size $N=1000$ with low and high clustering coefficients (networks with a high clustering coefficient were generated by using the method proposed by Kim (2004)). These authors found that in a clustered network the synchronization emerges at a lower coupling $J$ than a network with the same degree distribution but with a lower clustering coefficient. However, in the latter network the global synchronization is stronger.

Timme (2006) simulated the Kuramoto model on directed networks and observed a topologically induced transition from synchrony to disordered dynamics. This transition may be a general phenomenon for different types of dynamical models of synchronization on directed networks.

Synchronization of coupled oscillators in the $\mathrm{Ku}$ ramoto model to an external periodic input, called pacemaker, was studied for lattices, Cayley trees and complex networks by Kori and Mikhailov (2004, 2006); Radicchi and Meyer-Ortmanns (2006); Yamada (2002). This phenomenon is called entrainment. The pacemaker is assumed to be coupled with a finite number of vertices in a given network. Entrainment appears above a critical coupling strength $J_{c r}$. Kori and Mikhailov (2004) showed that $J_{c r}$ increases exponentially with increasing the mean shortest path distance $\mathcal{L}$ from the pacemaker to all vertices in the network, i.e., $J_{c r} \sim e^{a \mathcal{L}}$. In a complex network, $\mathcal{L}$ is proportional to the mean intervertex distance $\bar{\ell}(N)$ which, in turn, is typically proportional to $\ln N$, see Sec. II.A This leads to $J_{c r} \sim N^{b}$, where $b$ is a positive exponent. It was shown that frequency locking to the pacemaker strongly depends on its frequency and the network architecture.

\section{Coupled dynamical systems}

Consider $N$ identical dynamical systems. An individual system is described by a vector dynamical variable $\mathbf{x}_{i}(t), i=1, \ldots N$. The individual dynamics is governed by the equation: $\dot{\mathbf{x}}_{i}=\mathbf{F}\left(\mathbf{x}_{i}\right)$, where $\mathbf{F}$ is a vector function. These dynamical systems are coupled by edges and their dynamics is described by the equation:

$$
\dot{\mathbf{x}}_{i}=\mathbf{F}\left(\mathbf{x}_{i}\right)-J \sum_{j} L_{i j} \mathbf{H}\left(\mathbf{x}_{j}\right)
$$

where $J$ is the coupling strength, $\mathbf{H}\left(\mathbf{x}_{j}\right)$ is an output function which determines the effect of vertex $j$ on dynamics of vertex $i$. The network topology is encoded in the Laplacian matrix $L_{i j}=q_{i} \delta_{i j}-a_{i j}$, where $a_{i j}$ is the adjacency matrix, and $q_{i}$ is degree of vertex $i$. The Laplacian matrix is a zero-row-sum matrix, i.e., $\sum_{j} L_{i j}=0$ for all $i$. This property has the following consequence. Any solution of the equation $\dot{\mathbf{s}}=\mathbf{F}(\mathbf{s})$ is also a solution of Eq. (150), $\mathbf{x}_{i}=\mathbf{s}(t)$, i.e., dynamical systems evolve coherently.

\section{Stability criterion.}

We use the spectral properties of $L$ in order to determine the stability of the fully synchronized state against small perturbations, $\mathbf{x}_{i}=\mathbf{s}(t)+\eta_{i}$. The Laplacian has nonnegative eigenvalues which can be ordered as follows, $0=\lambda_{1}<\lambda_{2} \leqslant \ldots \leqslant \lambda_{N}$. The zero eigenvalue corresponds to the uniform eigenfunction, $f_{i}^{(0)}=1$ for all $i$ (the synchronized state). The remaining eigenfunctions 
$f_{i}^{(\lambda)}$ with $\lambda \geqslant \lambda_{2}$ are transverse to $f_{i}^{(0)}$. Representing a perturbation as a sum of the transversal modes, $\eta_{i}=\sum_{\lambda \geqslant \lambda_{2}} \eta_{\lambda} f_{i}^{(\lambda)}$, we find the master stability equation from Eq. (150):

$$
\dot{\eta}_{\lambda}=[D \mathbf{F}(\mathbf{s})-\alpha D \mathbf{H}(\mathbf{s})] \eta_{\lambda},
$$

where $\alpha=J \lambda . D \mathbf{F}$ and $D \mathbf{H}$ are the Jacobian matrices. If the largest Lyapunov exponent $\Lambda(\alpha)$ of this equation is negative, then the fully synchronized state is stable (Pecora and Carroll, 1998). $\Lambda(\alpha)$ is called the master stability function. This function is known for various oscillators such as Rössler, Lorenz, or double-scroll chaotic oscillators. Equation (151) is valid if the coupling matrix $L_{i j}$ is diagonalizable. A generalization of the master stability equation for non-diagonalizable networks (e.i., for the case of a non-symmetric coupling matrix) is given in Nishikawa and Motter (2006a, b) .

Thus we have the following criterion of the stability: the synchronized state is stable if and only if $\Lambda\left(J \lambda_{n}\right)<0$ for all $n=2, \ldots N$. In this case, a small perturbation $\eta_{\lambda}$ converges to zero exponentially as $t \rightarrow \infty$. The condition $\Lambda\left(J \lambda_{1}\right)=\Lambda(0)<0$ determines the dynamical stability of the solution $\mathbf{s}(t)$ to the individual dynamics.

Usually, the function $\Lambda(\alpha)$ is negative in a bound region $\alpha_{1}<\alpha<\alpha_{2}$. Therefore, a network is synchronizable if simultaneously $J \lambda_{2}>\alpha_{1}$ and $J \lambda_{N}<\alpha_{2}$. This is equivalent to the following condition:

$$
\frac{\lambda_{N}}{\lambda_{2}}<\frac{\alpha_{2}}{\alpha_{1}}
$$

(Barahona and Pecora, 2002). Note that $\lambda_{2}$ and $\lambda_{N}$ are completely determined by the network topology, while $\alpha_{1}$ and $\alpha_{2}$ depend on the specific dynamical functions $\mathbf{F}$ and $\mathbf{H}$. The value of $\alpha_{2} / \alpha$ typically ranges from 5 to 100 for various chaotic oscillators. The criterion Eq. (152) implies the existence of the interval $\left(\alpha_{1} / \lambda_{2}, \alpha_{2} / \lambda_{N}\right)$ of the coupling strength $J$ where the synchronization is stable. The smaller the eigenratio $\lambda_{N} / \lambda_{2}$, the larger this interval and the better synchronizability. If $J<\alpha_{1} / \lambda_{2}$, then modes with the small eigenvalues $\lambda<\alpha_{1} / J$ break down synchronization. If $J>\alpha_{2} / \lambda_{N}$, then modes with the large eigenvalues $\lambda>\alpha_{2} / J$ lead away from the synchronized state.

The spectrum of the Laplacian on the fully connected graph is simple: $\lambda_{1}=0$ and $\lambda_{2}=\ldots=\lambda_{N}=N$. The eigenratio $\lambda_{N} / \lambda_{2}$ is equal to 1 . It corresponds to the highest possible synchronizabilty. In the $d$-dimensional cubic lattice of side length $l=N^{1 / d}$, the minimum eigenvalue $\lambda_{2}$ of the Laplacian is small: $\lambda_{2} \propto l^{-2}$. On the other hand, the largest eigenvalue $\lambda_{N}$ is finite: $\lambda_{N} \sim d$. Therefore, the eigenratio $\lambda_{N} / \lambda_{2}$ diverges as $N \rightarrow \infty$. It means that the complete synchronization is impossible in an infinite $d$-dimensional lattice (Hong et al., 2004a; Wang and Chen, 2002). Only a finite lattice can be synchronized.

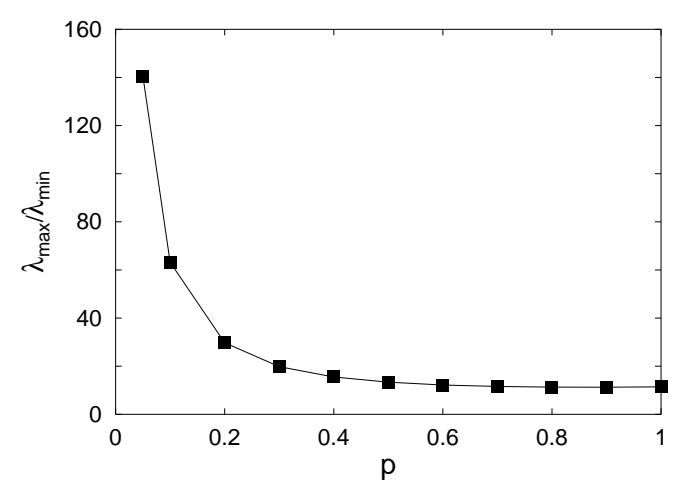

FIG. 38 Ratio $\lambda_{N} / \lambda_{2}$ versus the fraction of shortcuts, $p$, for the Watts-Strogatz network generated from a ring. Adapted from Hong et al. (2004a).

\section{Numerical study.}

Synchronization of coupled dynamical systems on various complex networks was extensively studied numerically. It turned out that the random addition of a small fraction of shortcuts, $p$, to a regular cubic lattice leads to a synchronizable network (Barahona and Pecora, 2002; Hong et al., 2004a; Wang and Chen, 2002). For example, a ring of $N$ vertices with shortcuts is always synchronizable if $N$ is sufficiently large. The shortcuts decrease sharply the ratio $\lambda_{N} / \lambda_{2}$ (see Fig. 38) until the network becomes synchronizable. The heuristic reason for this effect lies in the fact that adding shortcuts leads to the Watts-Strogatz network with the small-world effect. The average shortest path between two vertices chosen at random becomes very small compared to the original regular lattice. In other words, the small world effect improves synchronizability of the Watts-Strogatz network compared with a regular lattice.

Synchronization is also enhanced in other complex networks. One can show that the minimum eigenratio $\lambda_{N} / \lambda_{2}$ is achieved for the Erdős-Rényi graph. In scale-free networks, the eigenratio $\lambda_{N} / \lambda_{2}$ increases with decreasing degree distribution exponent $\gamma$, and so synchronizability becomes worse. This effect was explained by the increase of heterogeneity (Motter et al., 2005a, b; Nishikawa et al., 2003). It was found that a suppression of synchronization is related to the increase of the load on vertices. Importantly, the eigenratio $\lambda_{N} / \lambda_{2}$ increases strongly with $N$. Kim and Motter (2007), see also Motter (2007), found that the largest eigenvalue $\lambda_{N}$ in a uncorrelated scale-free network is determined by the cutoff of the degree distribution: $\lambda_{N}=q_{\text {cut }}+1$. The eigenvalue $\lambda_{2}$ is nearly size-independent and is ensemble averageable. (The last statement means that as $N \rightarrow \infty$, the ensemble distribution of $\lambda_{2}$ converges to a peaked distribution.) This leads to

$$
\frac{\lambda_{N}}{\lambda_{2}} \sim \min \left[N^{1 /(\gamma-1)}, N^{1 / 2}\right],
$$


see Sec. II.E.4. Therefore, it is difficult or even impossible to synchronize a large scale-free network with sufficiently small $\gamma$. These analytical results agree with numerical calculations of the Laplacian spectra of uncorrelated scale-free networks.

Another way to enhance synchronization is to use a network with asymmetric or weighted couplings. Motter et al. (2005a, b, c) considered an asymmetric degree dependent coupling matrix $q_{i}^{-\eta} L_{i j}$ instead of $L_{i j}$ in Eq. (150), where $\eta$ is a tunable parameter. Their numerical and analytical calculations demonstrated that if $\eta=1$, then in a given network topology the synchronizability is maximum and does not depend on the network size. In this case, the eigenratio $\lambda_{N} / \lambda_{2}$ is quite insensitive to the form of the degree distribution. Interestingly, in a random network the eigenvalues $\lambda_{2}$ and $\lambda_{N}$ of the normalized Laplacian matrix $q_{i}^{-1} L_{i j}$ achieve 1 as $\lambda_{2}=1-O(1 / \sqrt{\langle q\rangle})$ and $\lambda_{N}=1+O(1 / \sqrt{\langle q\rangle})$ in the limit of a large mean degree $\langle q\rangle \gg 1$ (Chung, 1997). Therefore in this limit the eigenratio $\lambda_{N} / \lambda_{2}$ is close to 1 , and the system is close to the highest possible synchronizability.

Note that apart the synchronization, network spectra have numerous applications to structural properties of networks and processes in them. For results on Laplacian spectra of complex networks and their applications, see, e.g., Chung (1997); Dorogovtsev et al. (2003); Kim and Motter (2007); Motter (2007) and references therein.

Chavez et al. (2005) found that a further enhancement of synchronization in scale-free networks can be achieved by scaling the coupling strength to the load of each edge. Recall that the load $l_{i j}$ of an edge $i j$ is the number of shortest paths which go through this edge. The authors replaced the Laplacian $L_{i j}$ to a zero row-sum matrix with off-diagonal elements $-l_{i j}^{\alpha} / \sum_{j \in N_{i}} l_{i j}^{\alpha}$, where $\alpha$ is a tunable parameter. This weighting procedure used a global information of network pathways. Chavez et al. (2005) demonstrated that varying the parameter $\alpha$, one may efficiently get better synchronization. A similar improvement was obtained by using a different, local weighting procedure based on the degrees of the nearest neighbors (Motter et al., 2005c). In networks with inhomogeneous couplings between oscillators, the intensity of a vertex is defined as the total strength of input couplings. Zhou et al. (2006) showed that the synchronizability in weighted random networks is enhanced as vertex intensities become more homogeneous.

The effect of degree correlations in a network on synchronization of coupled dynamical systems was revealed by Bernardo et al. (2007). These authors studied assortatively mixed scale-free networks. Their degree correlated networks were generated by using the method proposed by Newman (2003d). They showed that disassortative mixing (connections between high-degree and low-degree vertices are more probable) enhances synchronization in both weighted and unweighted scale-free networks compared to uncorrelated networks. However the synchronization in a correlated network depends on the a)

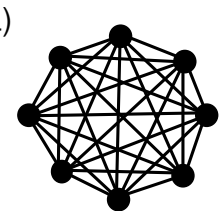

b)

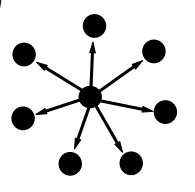

c)

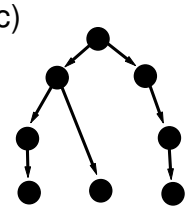

FIG. 39 Examples of graphs with optimal synchronizability: (a) a fully connected graph; (b) a directed star; (c) a hierarchical directed random graph.

weighting procedure (Chavez et al., 2006).

Above we showed that the fully connected graph gives the optimal synchronization. However this graph is costis-no-object and uncommon in nature. Which other architectures maximize the synchronizability of coupled dynamical systems? Nishikawa and Motter (2006a,b) came to the conclusion that the most optimal networks are directed and non-diagonalizable. Among the optimal networks they found a subclass of hierarchical networks with the following properties: (i) these networks embed an oriented spanning tree (i.e., there is a node from which all other vertices of the network can be reached by following directed links); (ii) there are no directed loops, and (iii) the total sum of input couplings at each vertex is the same for all vertices. Examples of optimal network topologies are shown in Fig. 39.

\section{SELF-ORGANIZED CRITICALITY PROBLEMS ON NETWORKS}

In this section we discuss avalanche processes in models defined on complex networks and other related phenomena.

\section{A. Sandpiles and avalanches}

The sandpile dynamics on the Erdős-Rényi random graphs was studied since (Bonabeau, 1995) but no essential difference from high-dimensional lattices was found. Goh et al. (2003); Lee et al. (2004a b) investigated a variation of the famous Bak-Tang-Wiesenfeld (BTW) model on scale-free uncorrelated networks and observed an effect of the network architecture on the self-organized criticality (SOC) phenomenon. Let us discuss these results.

The model is defined as follows. For each vertex $i$, a threshold $a_{i}=q_{i}^{1-\eta}$ is defined, where $0 \leq \eta \leq 1$, so that $a_{i} \leq q_{i}$. A number of grains at vertex $i$ is denoted by $h_{i}$.

(i) A grain is added to a randomly chosen vertex $i$, and $h_{i}$ increases by 1 .

(ii) If the resulting $h_{i}<a_{i}$, go to (i). On the other hand, if $h_{i} \geq a_{i}$, then $h_{i}$ is decreased by $\left\lceil a_{i}\right\rceil$, the smallest integer greater or equal to $a_{i}$. That is, $h_{i} \rightarrow h_{i}-\left\lceil a_{i}\right\rceil$. These $\left\lceil a_{i}\right\rceil$ toppled grains jump to 
$\left\lceil a_{i}\right\rceil$ randomly chosen nearest neighbors of vertex $i$ : $h_{j} \rightarrow h_{j}+1$.

(iii) If for all these $\left\lceil a_{i}\right\rceil$ vertices, the resulting $h_{j}<a_{j}$, then the "avalanche" process finishes. Otherwise, the vertices with $h_{j} \geq a_{j}$ are updated in parallel $(!), h_{j} \rightarrow\left\lceil a_{j}\right\rceil$, their randomly chosen neighbors receive grains, and so on until the avalanche stops. Then repeat (i).

Note that the particular, "deterministic" case of $\eta=0$, where all nearest neighbors of an activated vertex receive grains (as in the BTW model) essentially differs from the case of $\eta>0$, where $\left\lceil a_{i}\right\rceil<q_{i}$.

As is usual in SOC problems, the statistics of avalanches was studied: the size distribution $\mathcal{P}_{s}(s) \sim s^{-\tau}$ for the avalanches (the "size" is here the total number of toppling events in an avalanche) and the distribution $\mathcal{P}_{t}(t) \sim t^{-\delta}$ of their durations. (The distribution of the avalanche area - the number of vertices involved - is quite similar to $\mathcal{P}_{s}(s)$.) Taking into account the treelike structure of uncorrelated networks, one can see that (i) an avalanche in this model is a branching process, avalanches are trees, (ii) the duration of an avalanche $t$ is the distance from its root to its most remote vertex, and (iii) the standard technique for branching processes is applicable to this problem.

The basic characteristic of the avalanche tree is the distribution of branching, $p(q)$. According to Goh et al. (2005); Lee et al. (2004a, $\mathrm{b}), p(q)=p_{1}(q) p_{2}(q)$. The first factor is the probability that $q-1<a \leq q$, that is $q$ grains will fall from a vertex in the act of toppling. $p_{2}(q)$ is the probability that before the toppling, the vertex has exactly $q-1$ grains. The assumption that the distribution of $h$ is homogeneous gives the estimate $p_{2}(q) \sim 1 / q$. As for $p_{1}(q)$, one must take into account that (i) the degree distribution of an end of an edge is $q P(q) /\langle q\rangle$, (ii) $P(q) \sim q^{-\gamma}$, and (iii) $a=q^{1-\eta}$. As a result, $p_{1}(q) \sim q^{-(\gamma-1-\eta) /(1-\eta)}$. Thus, the distribution of branching is $p(q) \sim q^{-(\gamma-2 \eta) /(1-\eta)} \equiv q^{-\gamma^{\prime}}$. One can see that if $p_{2}(q)=1 / q$, then $\sum_{q} q p(q)=1$.

Goh et al. (2003); Lee et al. (2004a, b) applied the standard technique to the branching process with this $p(q)$ distribution and arrived at power-law size and duration distributions, which indicates the presence of a SOC phenomenon for the assumed threshold $a=q^{1-\eta}$. They obtained exponents $\tau$ and $\delta$. With these exponents, one can easily find the dynamic exponent $z=(\delta-1) /(\tau-1)$ (the standard SOC scaling relation), which in this case coincides with the fractal dimension of an avalanche. The results are as follows. There is a threshold value, $\gamma_{c}=3-\eta$, which separates two regimes:

$$
\begin{aligned}
& \text { if } \gamma>3-\eta, \quad \text { then } \tau=3 / 2, \quad \delta=z=2, \\
& \text { if } 2<\gamma<3-\eta, \quad \text { then } \tau=\frac{\gamma-2 \eta}{\gamma-1-\eta}, \delta=z=\frac{\gamma-1-\eta}{\gamma-2} .
\end{aligned}
$$

It is easy to understand these results for the fractal dimension of an avalanche, $z$. The reader may check that this $z$ exactly coincides with the fractal dimension of equilibrium connected trees with the degree (or branching) distribution equal to $p(q) \sim q^{-\gamma^{\prime}}$, see Sec. II.C.

For the numerical study of the BTW model on small-world networks, see de Arcangelis and Herrmann (2002). The BTW model is one of numerous SOC models. There were a few studies of other SOC models on complex networks. For example, for the Olami-Feder-Christensen model on various networks, see Caruso et al. (2006, 2007), and for a Manna type sandpile model on small-world networks, see Lahtinen et al. (2005). The Bak-Sneppen model on networks was studied in Kulkarni et al. (1999), Moreno and Vazquez (2002), Masuda et al. (2005), and Lee et al. (2005b).

\section{B. Cascading failures}

Devastating power blackouts are in the list of most impressive large-scale accidents in artificial networks. In fact, a blackout is a result of an avalanche of overload failures in power grids. A very simple though representative model of a cascade of overload failures was proposed by Motter and Lai (2002). The load of a vertex in this model is betweenness centrality - the number of the shortest paths between other vertices, passing through the vertex, Sec. II.A. Note that frequently the betweenness centrality is simply called load (Goh et al., 2001).

For every vertex $i$ in this model, a limiting loadcapacity is introduced:

$$
c_{i}=(1+\alpha) b_{0 i}
$$

where $b_{0 i}$ is the load (betweenness centrality) of this vertex in the undamaged network. The constant $\alpha \geq 0$ is a "tolerance parameter" showing how much an initial load can be exceeded. A cascading failure in this models looks as follows.

(i) Delete a vertex. This leads to the redistribution of loads of the other vertices: $b_{0 i} \rightarrow b_{0 i}^{\prime}$.

(ii) Delete all overloaded vertices, that is the vertices with $b_{0 i}^{\prime}>c_{i}$.

(iii) Repeat this procedure until no overloaded vertices remain.

In their simulations of various networks, Motter and Lai measured the ratio $G=N_{\text {after }} / N$, where $N$ and $N_{\text {after }}$ are, respectively, the original number of vertices in a network and the size of its largest connected component after the cascading failure. (Assume that the original network coincides with its giant connected component.) Resulting $G(\alpha)$ depend on (i) the architecture of a network, (ii) the parameter $\alpha$, and (iii) characteristics of the first failing vertex, e.g., on its degree.

In a random regular graph, for any $\alpha>0, G$ is 1 , and only if $\alpha=0$, the network will be completely destroyed, $G=0$. On the other hand, in networks with heavy-tailed 
degree distributions, $G$ strongly depends on the degree (or the load) of the first removed vertex. Motter and Lai used a scale-free network with $\gamma=3$ in their simulation. Let us briefly discuss their results. $\alpha=0$ gives $G=0$ for any starting vertex in any network, while $\alpha \rightarrow \infty$ results in $G=1$. The question is actually about the form of the monotonously growing curve $G(\alpha)$. When the first removed vertex is chosen at random, the cascade is large ( $G$ strongly differs from 1 ) only at small $\alpha$, and $G(\alpha)$ rapidly grows from 0 to 1 . If the first vertex is chosen from ones of the highest degrees, then $G$ gently rises with $\alpha$, and cascades may be giant even at rather large $\alpha$.

Lee et al. (2005a) numerically studied the statistics of the cascades in this model defined on a scale-free network with $2<\gamma \leq 3$ and found that in this case, there is a critical point $\alpha_{c} \approx 0.15$. At $a<a_{c}$, there are giant avalanches, and at $\alpha>\alpha_{c}$, the avalanches are finite. These authors observed that at the critical point, the size distribution of avalanches has a power-law form, $\mathcal{P}(s) \sim$ $s^{-\tau}$, where exponent $\tau \approx 2.1(1)$ in the whole range $2<$ $\gamma \leq 3$.

This model can be easily generalized: $\alpha$ may be defined as a random variable, instead of betweenness centrality other characteristics may be used, etc.- -see, e.g., Motter (2004) or, for a model with overloaded links, Moreno et al. (2003) and Bakke et al. (2006). Note that there are other approaches to cascading failures. For example, Watts (2002) proposed a model where, in simple terms, cascading failures were treated as a kind of epidemic outbreaks.

\section{Congestion}

Here we only touch upon basic models of jamming and congestion proposed by physicists. Ohira and Sawatari (1998) put forward a quite simple model of congestion. Originally it was defined on a lattice but it can be easily generalized to arbitrary network geometries.

The vertices in this model are of two types - hosts and routers. Hosts send packets at some rate $\lambda$ to other (randomly chosen) hosts, so that every packet has its own target. Each packet passes through a chain of routers storing and forwarding packets. There is a restriction: the routers can forward not more than one packet per time step. The routers are supposed to have infinite buffer space, where a queue of packets is stored. The packet at the head of the queue is sent first. A router sends a packet to that its neighboring router which is the closest to the target. If there occur more than one such routers, then one of them is selected by some special rules. For example, one may choose the router with the smallest flow of packets through it.

In their simulations Ohira and Sawatari studied the average time a packet needs to rich its target versus the packet injection rate $\lambda$. It turned out that this time strongly rises above some critical value $\lambda_{c}$, which indicates the transition to the congestion phase. The obser- vations of these authors suggest that it is a continuous transition, without a jump or hysteresis. The obvious reason for this jamming transition is the limited forwarding capabilities of routers - one packet per time step.

Solé and Valverde (2001) investigated this transition in the same model. They numerically studied the timeseries dynamics of the number of packets at individual routers, and found a set of power laws at the critical point. In particular, they observed a $1 / f$-type power spectrum of these series and a power-law distribution of queue lengths. [Similar critical effects were found in an analytically treatable model of traffic in networks with hierarchical branching, see Arenas et al. (2001).] They proposed the following idea. Since the traffic is most efficient at $\lambda_{c}$, the Internet self-organizes to operate at criticality. This results in various self-similar scaling phenomena in the Internet traffic.

These attractive ideas became the subject of strict criticism from computer scientists (Willinger et al., 2002). Let us dwell on this criticism, all the more so that it was from the discoverers of the scaling properties of the Internet traffic (Leland et al., 1994). They wrote: "selfsimilar scaling has been observed in networks with low, medium, or high loads, and any notion of a "magical" load scenario where the network has to run at critical rate $\lambda_{c}$ to show self-similar traffic characteristics is inconsistent with the measurements". They listed very simple alternative reasons for these self-similar phenomena. This criticism was, in fact, aimed at a wide circle of self-organized criticality models of various aspects of the real Internet, proposed by physicists. Willinger et al. stressed that these models "are only evocative; they are not explanatory". In their definition, an evocative model only "can reproduce the phenomenon of interest but does not necessarily capture and incorporate the true underlying cause". On the other hand, an explanatory model "also captures the causal mechanisms (why and how, in addition to what)." Ask yourself: how many explanatory models of real networks were proposed?

Guimerà et al. (2002) developed an analytical approach where search and congestion problems were interrelated. In their simple theory the mean queue length at vertices of a network was related to a search cost in this network. The latter is the mean number of steps needed to find a target vertex. In this approach, minimizing the mean queue length is reduced to minimizing the search cost. This approach was used to find optimal network architectures with minimum congestion.

Echenique et al. (2005) introduced a model of network traffic with a protocol allowing one to prevent and relieve congestion. In their model, routers forward packets, taking into account the queue lengths at their neighbors. Namely, a packet is sent to that neghboring router $j$, which has the minimum value

$$
\delta_{j} \equiv h \ell_{j t}+(1-h) c_{j} .
$$

Here $\ell_{j t}$ is the length of the shortest path from router $j$ to the target of the packet, $c_{j}$ is the queue length at the 


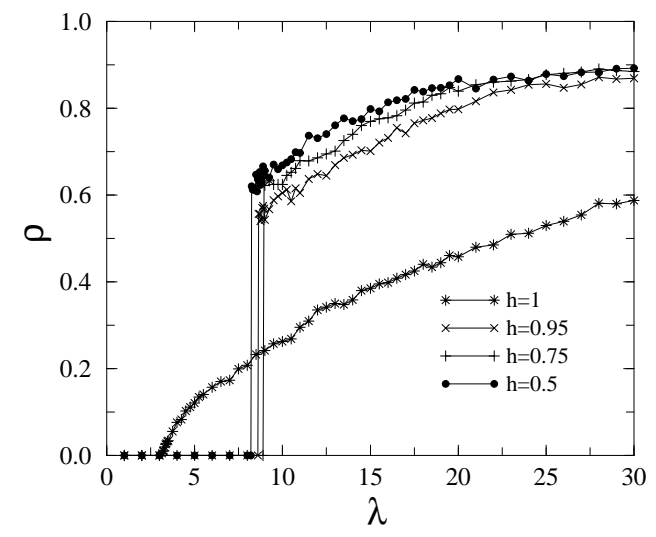

FIG. 40 Order parameter $\rho$ versus the packet injection rate $\lambda$ for varios $h$ in the model of Echenique et al. (2005). From Echenique et al. (2005).

router, and the parameter $h$ is in the range $0 \leq h \leq 1$. Echenique et al. performed the numerical simulations by using the map of a real Internet network but their results should be also valid for other architectures. As an order parameter for congestion they used the ratio: $\rho=$ (the number of packets that have not reached their targets during the observation)/(the total number of packets generated during this time period). It turned out that if the parameter $h$ is smaller than 1 , then the transition to the congestion phase occurs at an essentially higher rate $\lambda_{c}$. Furthermore, when $h<1$, the order parameter emerges with a jump as in a first order phase transition, while at $h=1$ the transition resembles a usual second order phase transition, see Fig. 40, Remarkably, the locations of these transitions, as well as the whole curves $\rho(\lambda)$, practically coincide at the studied $h=0.95,0.75,0.5$. On the other hand, the congestion $\rho$ at $h<1$ is much higher than at $h=1$ at the same $\lambda>\lambda_{c}$. The routing protocol of Echenique et al. was explored and generalized in a number of studies. For one of possible generalizations see Liu et al. (2006) and Zhang et al. (2007).

Another approach to network traffic, treating this process in terms of specific diffusion of packets, was developed by Tadić et al. (2007); Tadić and Thurner (2004); Tadić et al. (2004), see also Wang et al. (2006). The theory of this kind of traffic was elaborated by Fronczak and Fronczak (2007). Danila et al. (2006) studied routing based on local information. They considered "routing rules with different degrees of congestion awareness, ranging from random diffusion to rigid congestion-gradient driven flow". They found that the strictly congestion-gradient driven routing easily leads to jamming. Carmi et al. (2006a) presented a physical solution of the problem of effective routing with minimal memory resources. Toroczkai and Bassler (2004) investigated the influence of network architectures on the congestion. Helbing et al. (2007) described the generation of oscillations in network flows. Rosvall et al. (2004) dis- cussed how to use limited information to find the optimal routes in a network. For the problem of optimization of network flows, see also Gourley and Johnson (2006) and references therein.

\section{OTHER PROBLEMS AND APPLICATIONS}

In this section we briefly review a number of critical effects and processes in networks, which have been missed in the previous sections.

\section{A. Contact and reaction-diffusion processes}

\section{Contact process}

The contact process (Harris, 1974) is in a wide class of models exhibiting non-equilibrium phase transitions, for example, the SIS model of epidemics, which belong to the directed percolation universality class (Grassberger and de la Torre, 1979), see the review of Hinrichsen (2000). The contact process on a network is defined as follows. An initial population of particles occupies vertices in a network. Each vertex can be occupied by only one particle (or be empty). At each time step $t$, a particle on an arbitrary chosen vertex either (i) disappears with a probability $p$ or (ii) creates with the probability $1-p$ a new particle at an arbitrary chosen unoccupied neighboring vertex.

Let us introduce an average density $\rho_{q}(t)$ of particles at vertices with degree $q$. The time evolution of $\rho_{q}(t)$ is given by the mean-field rate equation:

$$
\frac{d \rho_{q}(t)}{d t}=-p \rho_{q}(t)+(1-p) q\left[1-\rho_{q}(t)\right] \sum_{q^{\prime}} \rho_{q^{\prime}}(t) \frac{P\left(q^{\prime} \mid q\right)}{q^{\prime}},
$$

where $P\left(q^{\prime} \mid q\right)$ is the conditional probability that a vertex of degree $q$ is connected to a vertex of degree $q^{\prime}$ (Castellano and Pastor-Satorras, 2006a). The first and second terms in Eq. (158) describe disappearance and creation of particles, respectively, at vertices with degree $q$. The factor $1 / q^{\prime}$ shows that a new particle is created with the same probability at any (unoccupied) nearest neighboring vertex of a vertex with degree $q^{\prime}$. Recall that in uncorrelated networks, $P\left(q^{\prime} \mid q\right)=q^{\prime} P\left(q^{\prime}\right) /\langle q\rangle$.

Equation (158) shows that if the probability $p$ is larger than a critical probability $p_{c}$, then any initial population of particles disappears at $t \rightarrow \infty$, because particles disappear faster then they are created. This is the so called absorbing phase. When $p<p_{c}$, an initial population of particles achieves a state with a non-zero average density:

$$
\rho=\sum_{q} P(q) \rho_{q}(t \rightarrow \infty) \propto \epsilon^{\beta},
$$

where $\epsilon=p_{c}-p$. This is the active phase. In the configuration model of uncorrelated random networks, the critical probability $p_{c}=1 / 2$ does not depend on the 
degree distribution while the critical exponent $\beta$ does. In networks with a finite second moment $\left\langle q^{2}\right\rangle$ we have $\beta=1$. If $\left\langle q^{2}\right\rangle \rightarrow \infty$, then $\beta$ depends on the asymptotic behavior of the degree distribution at $q \gg 1$. If the network is scale-free with $2<\gamma \leq 3$, the exponent $\beta$ is $1 /(\gamma-2)$. This critical behavior occurs in the infinite size limit, $N \rightarrow \infty$. In a finite network, $\rho$ is very small but finite at all $p>0$ and it is necessary to use the finite-size scaling theory.

Ha et al. (2007) and Hong et al. (2007a) applied the mean-field finite-size scaling theory to the contact process on finite networks (see Sec. IX.B). They showed that near the critical point $p_{c}$ the average density $\rho$ behaves as $\rho(\epsilon, N)=N^{-\beta / \bar{\nu}} f\left(\epsilon N^{1 / \bar{\nu}}\right)$, where $f(x)$ is a scaling function, the critical exponent $\beta$ is the same as above. The critical exponent $\bar{\nu}$ depends on degree distribution: $\bar{\nu}(\gamma>3)=2$, and $\bar{\nu}(2<\gamma \leq 3)=(\gamma-1) /(\gamma-2)$. The authors carried out Monte Carlo simulations of the contact process on the configuration model of uncorrelated scale-free networks of size to $N=10^{7}$. These simulations agreed well with the predictions of the meanfield scaling theory in contrast to earlier calculations of Castellano and Pastor-Satorras (2006a, 2007b).

Based on the phenomenological theory of equilibrium critical phenomena in complex networks (Sec. IX.A), Hong et al. (2007a) proposed a phenomenological meanfield Langevin equation which describes the average density of particles in the contact process on uncorrelated scale-free networks near the critical point:

$$
\frac{d \rho(t)}{d t}=\epsilon \rho-b \rho^{2}-d \rho^{\gamma-1}+\sqrt{\rho} \eta(t)
$$

where $\eta(t)$ is the Gaussian noise, $b$ and $d$ are constants. Note that the contact process contains the so-called multiplicative noise $\sqrt{\rho} \eta(t)$, in contrast to an equilibrium process with a thermal Gaussian noise (see, e.g., Hinrichsen (2000)). Neglecting the noise in Eq. (160), in the steady state one can obtain the critical behavior of $\rho$ and a finite-size scaling behavior of the relaxation rate (Sec. IX.B). As is natural, when a degree distribution is rapidly decreasing, this finite-size scaling coincides with that for the contact process on high-dimensional lattices (Lübeck and Janssen, 2005).

The time evolution of the average density $\rho(t)$ was studied by Castellano and Pastor-Satorras (2006a, 2007b) and Hong et al. (2007a). When $p \neq p_{c}$, in an infinite network, an initial population of particles exponentially relaxes to a steady distribution. The relaxation time $t_{c}$ is finite. At the critical point, $p=p_{c}$, the characteristic time $t_{c}$ diverges, and an initial distribution decays as $\rho(t) \sim t^{-\theta}$. Exponent $\theta=1$ for an uncorrelated complex network with a finite second moment $\left\langle q^{2}\right\rangle$, and $\theta=1 /(\gamma-2)$ for a scale-free network with $2<\gamma<3$. In a finite network, $t_{c}(N)$ is finite even at the critical point. In uncorrelated networks with $\left\langle q^{2}\right\rangle<\infty$, the character-

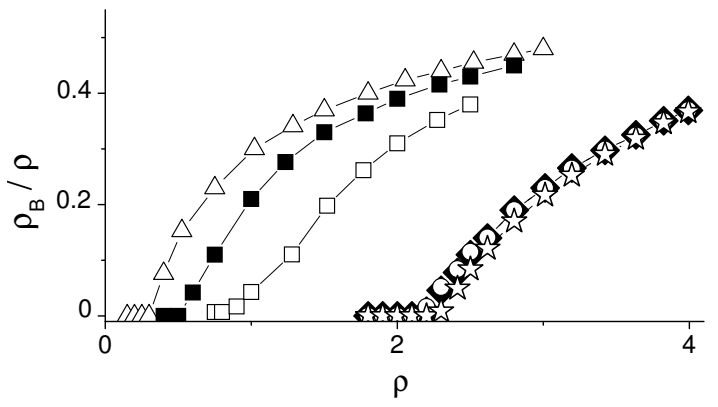

FIG. 41 Relative density of particles $B$ versus the total density of $A$ and $B$ particles in the reaction-diffusion model in scale-free networks at $\mu / \lambda=2$. Rightmost curves ( $A$ particles are non-diffusing): stars, $N=10^{4}, \gamma=3$; closed diamonds, $N=10^{4}, \gamma=2.5$; open circles, $N=10^{5}, \gamma=2.5$. Leftmost curves (both $A$ and $B$ particles are diffusing, $\gamma=2.5$ ): open squares, $N=10^{3}$; closed squares, $N=10^{4}$; open triangles, $N=10^{5}$. Adapted from Colizza et al. (2007).

istic relaxation time is

$$
t_{c} \sim \sqrt{\frac{N\langle q\rangle^{2}}{\left\langle q^{2}\right\rangle}}
$$

(Castellano and Pastor-Satorras, 2007b). Note that when exponent $\gamma>3$, the phenomenological approach based on Eq. (160) also leads to $t_{c} \propto N^{1 / 2}$. The size dependence of $t_{c}$ in the range $2<\gamma<3$, where $\left\langle q^{2}\right\rangle$ depends on $N$, is still under discussion, see Castellano and Pastor-Satorras (2007b); Hong et al. (2007a).

Giuraniuc et al. (2006) considered the contact process with a degree dependent rate of creation of particles assumed to be proportional to $\left(q_{i} q_{j}\right)^{-\mu}$, where $\mu$ is a tunable parameter, $q_{i}$ and $q_{j}$ are degrees of neighboring vertices. Using a mean-field approximation which is equivalent to the annealed network approximation, they showed that this degree dependent rate changes the critical behavior of the contact process in scale-free networks. The result is the shift of degree distribution exponent $\gamma$ to $\gamma^{\prime}=(\gamma-\mu) /(\gamma-1)$. This effect is similar to the Ising model with degree dependent interactions in Sec. VI.C.5. For finite-size scaling in contact processes with this degree-dependent rate of creation, see Karsai et al. (2006).

\section{Reaction-diffusion processes}

Reaction-diffusion processes on uncorrelated random complex networks were studied by Colizza et al. (2007). Consider the following process for particles of two types, $A$ and $B$. In an initial state, particles are distributed randomly over vertices of a network. There may be an arbitrary number of these particles at any vertex. Suppose that only particles at the same vertex may react and 
transform to other particles. The rules of this transformation are the following:

(i) Each particle $B$ can spontaneously turn into a $A$ particle at the same vertex at a rate $\mu: B \rightarrow A$.

(ii) Particles $A$ and $B$ can transform into two $B$ particles at the same vertex at a rate $\lambda: A+B \rightarrow 2 B$.

(iii) Particles $B$ can hop to neighboring vertices at the unit rate.

These reactions preserve the total number of particles in the system. The steady state of this process strongly depends on a supposed behavior of particles $A$.

If $A$ particles are non-diffusing and the total density of $A$ and $B$ particles, $\rho$, is smaller than the critical density $\rho_{c}=\mu / \lambda$, then $B$ particles disappear in the limit $t \rightarrow \infty$ (this is the absorbing phase). At $\rho>\rho_{c}$ there is a nonzero density of $B$ particles, $\rho_{B}$, in the steady state (this is the active phase), see Fig. 41. Colizza et al. (2007) showed that $\rho_{c}$ and the critical behavior do not depend on the degree distribution.

If $A$ particles can also hop, then the phase transition into the active phase occurs at a degree dependent critical density $\rho_{c}=\langle q\rangle^{2} \mu /\left(\left\langle q^{2}\right\rangle \lambda\right)$. In networks with divergent $\left\langle q^{2}\right\rangle, \rho_{c}$ is zero in the limit $N \rightarrow \infty$, see Fig. 41] A similar disappearance of the critical threshold was observed in percolation and the spread of diseases.

Network topology strongly affects dynamics of the diffusion-annihilation process. This process is defined in the following way. Identical particles diffuse in a network. If two particles are at the same vertex, they annihilate $(A+A \rightarrow \varnothing)$. Catanzaro et al. (2005) within the mean-field theory showed that in infinite uncorrelated random networks the average density of particles, $\rho(t)$, decreases as $t^{-\alpha}$ at large times, where the exponent $\alpha=1$ for a network with a finite second moment $\left\langle q^{2}\right\rangle$, and $\alpha=1 /(\gamma-2)$ for an uncorrelated scale-free network with degree distribution exponent $2<\gamma<3$ (i.e., with divergent $\left\langle q^{2}\right\rangle$ ). However, in a finite scale-free network, there is a crossover to the traditional mean field behavior $1 / t$ at times $t>t_{c}(N)$, where the crossover time $t_{c}(N)$ increases with increasing $N$. Thus the non-mean-field behavior with $\alpha=1 /(\gamma-2)$ may be observed only in a sufficiently large network (see ben-Avraham and Glasser (2007) for a discussion of kinetics of coalescence, $A+A \rightarrow$ $A$, and annihilation, $A+A \rightarrow \varnothing$, beyond the meanfield approximation in the Bethe lattice). This agrees with numerical simulations of Catanzaro et al. (2005); Gallos and Argyrakis (2004).

\section{B. Zero-range processes}

Zero-range process describes non-equilibrium dynamics of condensation of interacting particles in lattices and networks. This process is closely related to the ballsin-boxes model (Bialas et al., 1997) and equilibrium network ensembles (Angel et al., 2005, 2006; Burda et al.,
2001; Dorogovtsev et al., 2003b) discussed in Sec. IV.A. The interested reader will find a review of several applications of this model in Evans and Hanney (2005).

In the zero-range process, identical particles hop between vertices on a graph with a rate $u(n)$ which depends on the number of particles, $n$, at the vertex of departure. The total number of particles is conserved. In fact, an interaction between particles on the same vertex is encoded in the function $u(n)$. The case $u(n) \propto n$ corresponds to noninteracting particles. If $u(n)$ increases faster than $n$, then we deal with a local repulsion. If $u(n)$ decreases with $n$, then it assumes a local attraction. Emergence of the condensation depends on the hop rate $u(n)$ and the network structure.

The system evolves from an initial distribution of particles to a steady state. At a certain condition, the condensation of a finite fraction of particles occurs onto a single vertex. Note that this non-equilibrium phase transition occurs even in a one-dimensional lattice. In the steady state the distribution of particles over vertices can be found exactly. The probability that vertices $i=1,2, \ldots N$ are occupied by $n_{1}, n_{2}, \ldots n_{N}$ particles is

$$
\mathcal{P}\left(n_{1}, n_{2}, \ldots n_{N}\right)=A \prod_{i=1}^{N} f_{i}\left(n_{i}\right),
$$

where $A$ is a normalization constant, the function $f_{i}(n) \equiv \prod_{m-1}^{n}\left[\omega_{i} / u(m)\right]$ for $n \geq 1$, and $f_{i}(0) \equiv 1$ (Evans and Hanney, 2005). The parameters $\omega_{i}$ are the steady state weights of a single random walker which moves on a given network. In simple terms, the frequency of visits of the walker to a vertex is proportional to its weight. The weights satisfy the equation $\omega_{i}=\sum_{j} \omega_{j} T_{j i}$, where $T_{j i}$ is a rate of particle hops from vertex $j$ to neighboring vertex $i$. Using the function Eq. (162), one can find exact mean occupation numbers of vertices.

Let us first consider a homogeneous system where all vertices have the same degree. The condensation is absent if $u(n \rightarrow \infty) \rightarrow \infty$. In the steady state all vertices have the same average occupation number (this is the so called fluid phase). The condensation occurs if $u(n)$ decays asymptotically as $u(\infty)(1+b / n)$ with $b>2$. In this case, the steady state with the condensate emerges when the concentration of particles $\rho$ is larger than a critical concentration $\rho_{c}$ determined by the function $u(n)$. In the condensed phase, a finite fraction of particles, $\rho-\rho_{c}$, occupies a single vertex chosen at random. All other vertices are occupied uniformly with the mean occupation number $\rho_{c}$. If $u(n \rightarrow \infty)=0$, then $\rho_{c}=0$.

The zero-range process in uncorrelated scale-free networks with degree distribution exponent $\gamma>2$ was studied by Noh (2005); Noh et al. $(2005)$. The authors considered the case when the function $u(n)=n^{\delta}$. A particle can hop with the same probability to any nearest neighboring vertex, i.e., the transition probability $T_{i j}=1 / q_{i}$, where $q_{i}$ is degree of departure vertex $i$. In this case $\omega_{i}=q_{i}$. It was shown that if $\delta>\delta_{c}=1 /(\gamma-2)$, then the steady state is the fluid phase at any density of particles. If $\delta \leq \delta_{c}$, then the critical concentration $\rho_{c}=0$. At 
$\rho>0$, in the steady state, almost all particles are condensed not at a single vertex but a set of vertices with degrees exceeding $q_{c} \equiv\left[q_{\text {cut }}(N)\right]^{1-\delta / \delta_{c}}$. These vertices form a vanishingly small fraction of vertices in the network in the limit $N \rightarrow \infty$. Note that these results were obtained for the cutoff $q_{\text {cut }}(N)=N^{1 /(\gamma-1)}$ (see the discussion of $q_{\text {cut }}(N)$ in Sec. II.E.4). When $\delta=0$, then $q_{c}=q_{\text {cut }}(N)$ and all particles condense at a vertex with the highest degree $q_{\text {cut }}(N)$. See Tang et al. (2006) for specifics of condensation in a zero-range process in weighted scalefree networks.

The steady state in the zero-range process on a scalefree network is completely determined by the degree distribution. The topological structure plays no role (i.e., it does not matter whether vertices are arranged in a finite dimensional system or form a small world). It is assumed that the network structure may influence relaxation dynamics of the model, unfortunately, no exact results are known. Noh (2005) studied the evolution of an initial distribution of particles to the steady state and estimated the relaxation time $\tau$. In an uncorrelated random scale-free network, the relaxation time is $\tau \sim N^{z}$, where $z=\gamma /(\gamma-1)-\delta$, while in clustered scale-free networks, this exponent is $z=1-\delta$. This estimate agrees with numerical simulations in Noh (2005); Noh et al. (2005). Note that the scaling relation $\tau \sim N^{z}$ is also valid for a $d$ dimensional lattice. In this case the exponent $z$ depends on both the dimension $d$ and the probability distribution of hopping rates. Particularly, $z=2$ for a $d>2$-regular lattice (Evans and Hanney, 2005).

In a finite network, the condensate at a given vertex exists a finite time $\tau_{m}(N)$. After "melting" at this vertex, the condensate appears at another vertex, then - at another one, and so on. For a homogeneous network, e.g., for a random regular graph, $\tau_{m} \sim N^{z^{\prime}} \gg \tau \sim N^{z}$, where $z^{\prime}>z$. Bogacz et al. (2007a b); Waclaw et al. (2007) argued that in heterogeneous systems the typical melting time of the condensate, $\tau_{m}(N)$, increases exponentially with $N$, i.e., $\tau_{m}(N) \sim e^{c N}$, in contrast to a homogeneous system. The zero-range process relaxes slowly to the condensed phase in comparison to a relaxation time to the equilibrium state in the ferromagnetic Ising model. (The relaxation time of the Ising model is finite at all temperatures except the critical one, at which it scales with $N$ as $N^{s}$, see Sec.IX.B.) As soon as the condensate is formed, it exists for an exponentially long time at a vertex of the network: $\tau_{m} \sim e^{c N} \gg \tau \sim N^{z}$.

\section{The voter model}

According to Sood and Redner (2005), "the voter model is perhaps the simplest and most completely solved example of cooperative behavior". In this model, each vertex is in one of two states - spin up or spin down. In the vertex update version of the model, the evolution is defined as follows. At each time step,

(i) choose a vertex at random and (ii) ascribe to this vertex the state of its randomly chosen neighbor.

The evolution in the voter model starts with some random configuration of up and down spins, say, with a fraction of $n_{0}$ spins up. One can see that this evolution is determined by random annihilation of chaotic interfaces between "domains" with up and down spins. In a finite system, there is always a chance that the system will reach an absorbing state, where all spins up (or down). However, on the infinite regular lattices of dimensionality greater than 2 , the voter model never reaches the absorbing states staying in the active state for ever. For the voter model on the finite regular lattices of dimensionality greater than 2, the mean time to reach consensus is $\tau_{N} \sim N$ (ben-Avraham et al., 1990; Krapivsky, 1992). Here $N$ is the total number of vertices in a lattice.

On the other hand, the infinite one-dimensional voter model evolves to consensus. Castellano et al. (2003) and Vilone and Castellano (2004) studied the voter model on the Watts-Strogatz small-world networks and found that even a small concentration of shortcuts makes consensus unreachable in the infinite networks. This is quite natural, since these networks are infinite-dimensional objects.

It is important that the average fraction of spins up, $n$, conserves in the voter model on regular lattices, i.e., $n(t)=n_{0}=$ const. Here the averaging is over all initial spin configurations and over all evolution histories. Suchecki et al. (2005a b) found that on random networks, $n(t)$ is not conserved. Instead, the following weighted quantity conserves:

$$
\tilde{n}=\sum_{q} \frac{q P(q)}{\langle q\rangle} n(q)
$$

where $n(q)$ is the average fraction of spins up among vertices of degree $q$. Thus, $\tilde{n}(t)=\tilde{n}_{0}=$ const, where $\tilde{n}_{0} \equiv \tilde{n}(t=0)$. Note that $\tilde{n}$ is actually the probability that an end vertex of a randomly chosen edge is in state up.

Based on this conservation, Sood and Redner (2005) arrived at the following physical picture for the voter model on uncorrelated complex networks. Consensus is unreachable if these networks are infinite. In the finite networks, the mean time to reach consensus is finite. The evolution consists of two stages. The first is a short initial transient to an active state where at any particular evolution history, the fraction of vertices of a given degree with spin up is approximately $\tilde{n}_{0}$. In the slow second stage, coarsening develops, and the system has an increasing chance to approach consensus. The mean time to reach consensus is

$$
\tau_{N}=N \frac{\langle q\rangle^{2}}{\left\langle q^{2}\right\rangle}\left[\left(1-\tilde{n}_{0}\right) \ln \left(1-\tilde{n}_{0}\right)^{-1}+\tilde{n}_{0} \ln \tilde{n}_{0}^{-1}\right]
$$

So, the theory of Sood and Redner gives (a) $\tau_{N} \sim N$ for uncorrelated networks with a converging second moment of a degree distribution, (b) $\tau_{N} \sim N / \ln N$ in the case of 
the degree distribution $P(q) \sim q^{-3}$, and (c) $\tau_{N}$ growing slower than $N$ if $\left\langle q^{2}\right\rangle$ diverges, i.e., if the degree distribution exponent is less than 3 . In the last case, this size dependence (a power of $N$ with exponent less than 1 ) is determined by a specific model-dependent cutoff of the degree distribution, $q_{\text {cut }}(N)$.

Interestingly, in the second version of the voter model - edge update - the average fraction of up vertices is conserved as well as the mean magnetization. In the edge update voter model, at each time step, an end vertex of a randomly chosen edge adopts the state of the second end. In this model, the evolution of the system on a complex network is qualitatively the same as on high-dimensional regular lattices, and $\tau_{N} \sim N$ (Suchecki et al., 2005a , b).

Other basic types of spin dynamics are also widely discussed. Castellano et al. (2005) studied a difference between the voter dynamics and the Glauber-Metropolis zero-temperature dynamics on networks (Castellano and Pastor-Satorras, 2006b; Zhou and Lipowsky, 2005). In the Glauber-Metropolis dynamics in application to the Ising model at zero temperature, at each time step, a randomly chosen spin gets an energetically favorable value, +1 or -1 . In contrast to the evolution due to the interface annihilation in the voter model, in the Glauber-Metropolis dynamics, domain walls shorten diminishing surface tension. Svenson (2001) showed numerically that in infinite random networks, the Glauber-Metropolis dynamics of the Ising model at zero temperature does not reach the ground state. Häggström (2002) rigorously proved that this is true at least in the case of the Gilbert model of classical random graphs. Thus, this kind of dynamics can result in consensus only in finite networks, as in the voter model. Nonetheless, Castellano, et al. found that the voter and Glauber-Metropolis dynamics provide markedly different relaxation of spin systems on random networks. For the Glauber-Metropolis dynamics, the time dependence of the probability that a system does not yet reach consensus essentially deviates from exponential relaxation, typical for the voter dynamics.

For detailed discussion of the voter model on complex networks in context of opinion formation, see Wu and Huberman (2004). For other nonequilibrium phenomena in complex networks modeling social interactions, see, e.g., Klemm et al. (2003), Antal et al. (2005), and Baronchelli et al. (2007).

A few numerical studies were devoted to nonequilibrium phase transitions in the ferromagnetic Ising model on directed complex networks with possible application to processes in social, economic, and biological systems. In the directed Ising model, the interactions between spins are asymmetric and directed, so a Hamiltonian formulation is impossible. Each spin is affected only by those of its nearest neighboring spins which are connected to this spin by, say, outgoing edges. Using a directed Watts-Strogatz network generated from a square lattice, Sánchez et al. (2002) found that a ferromagnetic phase transition in this system is continuous at a sufficiently small density of the shortcuts. This transition, however, becomes of the first order above a critical concentration of the shortcuts. Lima and Stauffer (2006) carried out simulations of the ferromagnetic Ising model on a directed Barabási-Albert network at $T=0$ and found that different dynamics algorithms lead to different final states of the spin system. These first investigations demonstrate a strong influence of a directed network structure on the non-equilibrium dynamics. However, these systems are not understood as yet.

\section{Co-evolution models}

We mostly discuss systems where a cooperative model does not influence its network substrate. Holme and Newman (2006) described a very interesting contrasting situation, where an evolving network and interacting agents on it strongly influence each other. The model of Holme and Newman, in essence, is an adaptive voter model and may be formulated as follows. There is a sparse network of $N$ vertices with a mean degree $\langle q\rangle$. Each vertex may be in one of $G$ states- "opinions", where $G$ is a large number (which is needed for a sharp phase transition). Vertices and connections evolve: at each time step, choose a random vertex $i$ in state $g_{i}$. If the vertex is isolated, do nothing. Otherwise,

(i) with probability $\phi$, reattach the other end of a randomly chosen edge of vertex $i$ to a randomly chosen vertex with the same opinion $g_{i}$; or

(ii) with probability $1-\phi$, ascribe the opinion $g_{i}$ to a randomly chosen nearest neighbor $j$ of vertex $i$.

Due to process (i), vertices with similar opinions become connected - agents influence the structure of the network. Due to process (ii), opinions of neighbors change - the network influences agents.

Suppose that the initial state is the classical random graph with vertices in random states. Let the mean degree be greater than 1, so that the giant connected component is present. This system evolves to a final state consisting of a set of connected components, with all vertices in each of the components being in coinciding states - internal consensus. Of course, vertices in different connected components may be in different states. In their simulation, Holme and Newman studied the structure of this final state at various values of the parameter $\phi$. In more precise terms, they investigated the resulting size distribution $P(s)$ of the connected components.

If $\phi=0$, the connections do not move, and the structure of the final network coincides with the original one, with the giant connected component. This giant component is destroyed by process (i) if the probability $\phi$ is sufficiently high, and at $\phi \sim 1$, the network is segregated into a set of finite connected components, each one of about $N / G$ vertices. It turns out that at some critical 
value $\phi_{c}=\phi_{c}(\langle q\rangle, N / G)$ there is a sharp transition, where the giant connected component disappears. At the critical point, $P(s)$ seems to have a power-law form with a nonstandard exponent. There is a principle difference from the usual birth of the giant connected component in random networks - in this evolving system, the phase transition is nonequilibrium. In particular, this transition depends on the initial state of the system. We expect that models of this kind will attract much interest in the future, see works of Caldarelli et al. (2006); Ehrhardt et al. (2006); Gil and Zanette (2006); Kozma and Barrat (2007); Zanette (2007); Zimmermann et al. (2004), and the review of Gross and Blasius (2007). Allahverdvan and Petrosvan (2006) and Biely et al. (2007) considered somewhat related problems where spins at vertices and edges interacted with each other.

\section{E. Localization transitions}

In this subsection we briefly discuss two quite different localization problems - quantum and classical.

\section{Quantum localization}

Here we touch upon the transition from localized states of an electron on the Erdös-Rényi graph, that is the quantum percolation problem. The set of corresponding eigenfunctions $\psi(i, E)$, where $E$ is the energy described by the hopping Hamiltonian, obeys the equations:

$$
E \psi(i, E)=\sum_{j} a_{i j} \psi(j, E)
$$

where $a_{i j}$ are elements of the adjacency matrix. So, the quantum percolation problem is in fact the problem of the structure of eigenvectors of the adjacency matrix and its spectrum.

In the phase with delocalized states, the spectrum $(|E|<C\langle q\rangle$, where $C$ is some positive constant) is organized as follows (Harris, 1982). All states with $E_{c}(\langle q\rangle)<$ $|E|<C\langle q\rangle$ are localized, where $E_{c}$ is the mobility edge energy. On the other hand, in the range $|E|<E_{c}(\langle q\rangle)$, both localized and extended are present. At the localization threshold, $E_{c}$ becomes zero, and, as is natural, all the states are localized in the localization phase. This picture allows one to find the localization threshold by investigating only the zero energy states, since extended states first emerge at zero energy.

Harris (1982) (see also references therein) explained how to distinguish localized and extended states in the spectrum and how to relate the quantum percolation problem to classical percolation. It is important that he showed that the delocalization point, $q_{\text {deloc }}$, does not coincide with the classical percolation threshold (i.e., the point of the birth of the giant connected component, which is $\langle q\rangle=1$ in the Erdös-Rényi model).
Bauer and Golinelli (2001b) showed that the localization phase is at $\langle q\rangle\left\langle q_{\text {deloc }}=1.421529 \ldots\right.$. and above $q_{\text {deloc }}$ the conducting phase is situated. They also revealed another, relocalization transition at a higher mean degree, $q_{\text {reloc }}=3.154985 \ldots$. This intriguing relocalization was observed only in this work.

For numerical study of quantum localization in scalefree networks, which is a pretty difficult task, see Sade et al. (2005). This problem was not studied analytically.

\section{Biased random walks}

Let a classical particle randomly walk on a graph. It is well known that on $d$-dimensional lattices, (i) if $d \leq 2$, a walk is recurrent, that is a drunkard almost surely will get back to his home - "localization"; and (ii) if $d>2$, a walk is transient, that is with finite probability, it goes to infinity without returning to a starting point. Thus the dimension $d=2$ may be interpreted as a "localization transition".

Lyons (1990) found and analytically described a very similar transition in random networks, see also Lyons et al. (1996). Actually he considered random growing trees with a given distribution of branching, but Sood and Grassberger (2007) showed that in networks with locally tree-like structure, nearly the same conclusions hold. For brevity, let the network be uncorrelated. Consider a random walk started from a randomly chosen vertex 0, assuming that there is an "exponential" bias in the direction of vertex 0 . One may easily arrange this bias by labelling all vertices in the network by their shortest path distance to the starting vertex. Suppose that the probabilities of a jump of the walker from vertex $i$ ( $\ell$ steps from vertex 0$)$ to its nearest neighbors at distances $\ell-1, \ell$, or $\ell+1$ are related in the following way:

$$
\frac{p(i ; \ell \rightarrow \ell-1)}{p(i ; \ell \rightarrow \ell)}=\frac{p(i ; \ell \rightarrow \ell)}{p(i ; \ell \rightarrow \ell+1)}=\sqrt{\lambda}
$$

Then the localization transition is at $\lambda_{c}$, coinciding with the mean branching coefficient $B$, which is, as we know, $B=z_{2} / z_{1}=\left(\left\langle q^{2}\right\rangle-\langle q\rangle\right) /\langle q\rangle$ for the configuration model. It is exactly the same critical point as was observed in cooperative models on this network, see Secs. III.B.3 and VI.C.4 which indicates a close relation between these two classes of problems.

For $1 \leq \lambda<\lambda_{c}$, the average return time grows proportionally to $N^{\epsilon}$ with exponent $\epsilon=\ln (B / \lambda) / \ln B$, which is the analytical result of Bénichou and Voituriez (2007). At $\lambda=\lambda_{c}$, this time is $\propto \ln N$ as in the unbiased random walks on the chain of length $\ln N$ with the reflecting boundaries. Finally, for $\lambda>\lambda_{c}$, above the critical bias, the mean return time approaches a finite value at large $N$. Remarkably, the average return time coincides with the mean correlation volume $\bar{V}$, Sec. III.B.3 if the parameter $b$ characterizing the decay of correlations is taken 
to be $b=1 / \lambda$.

Sood and Grassberger (2007) measured the distribution of return times and found that due to the absence of small loops in the network, returns with sufficiently short odd times are virtually absent for any bias. In other words, in this range of times, a walker may get back to the starting vertex only by the same way he walked away.

\section{F. Decentralized search}

Recall that in the Watts-Strogatz small-world networks with variation of the number of shortcuts, there is a smooth crossover from a lattice (large world) to a small world. In marked contrast to this are Kleinberg's networks described in Sec. II.I as well as the long-range percolation problem. In these systems there is a sharp transition between the lattice and small-world geometries at some special value of the control parameterexponent $\alpha$, which depends on the dimensionality $d$ the lattice substrate (Beniamini and Berger, 2001; Biskup, 2004; Martel and Nguven, 2004). In these works actually a closely related long-range percolation problem was analysed. Assuming that the number of shortcuts is $O(N)$, i.e., the network is sparse, gives the following mean intervertex distances:

(i) for $\alpha<d, \bar{\ell}(N) \sim \ln N$;

(ii) for $d<\alpha<2 d, \bar{\ell}(N) \sim(\ln N)^{\delta(\alpha)}$, where $\delta(\alpha) \cong$ $\ln 2 / \ln (2 d / \alpha)>1$

(iii) for $\alpha>2 d, \bar{\ell}(N) \sim c(\alpha) N$, where $c(\alpha)$ depends only on $\alpha$.

Thus, there is a sharp transition from a "large world" to a "small world" at $\alpha=2 d$. (Note, however, that Moukarzel and de Menezes (2002) presented heuristic and numerical arguments that this transition is at $\alpha=d$, and $\bar{\ell} \sim N^{\mu(\alpha)}$ for $d<\alpha<2 d$, where $0<\mu(\alpha)<$ $1 / d$. The reason for this difference between two groups of results is not clear.) For other networks with a similar transition, see Hinczewski and Berker (2006) and Holme (2007).

The sparse network with exponent $\alpha$ equal to $d$ is unique in the following respect described by Kleinberg (1999, 2000, 2006). Kleinberg asked: how many steps in average, $\bar{\tau}(N)>\bar{\ell}(N)$, it will take to approach/find a target from an arbitrary vertex by using the fast "decentralized search greedy algorithm"? This algorithm exploits some information about geographic positions of vertices: at each step, move to the nearest neighbor (including the neighbors through shortcuts) geographically closest to the target [for other search algorithms based on local information, see Adamic et al. (2003)]. In particular, for $d=2$ :

(i) for $0 \leq \alpha<2, \bar{\tau}(N) \sim N^{(2-\alpha) / 6}$;

(ii) for $\alpha=2, \bar{\tau}(N) \sim \ln ^{2} N$ (which is also valid for general $\alpha=d)$;

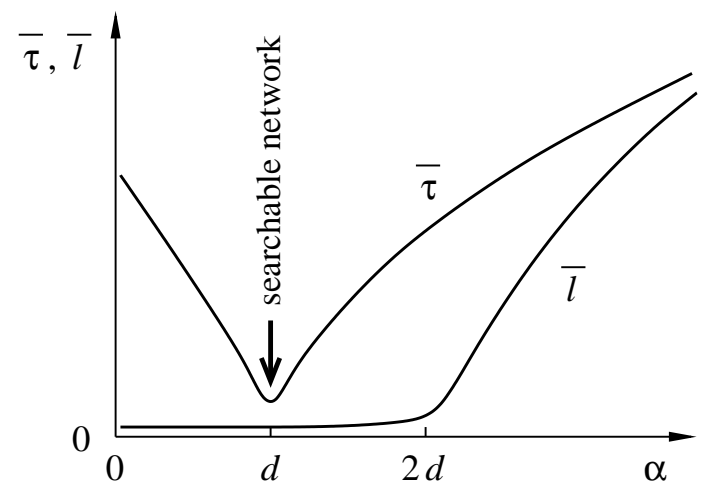

FIG. 42 Schematic plot of the mean intervertex distance $\bar{\ell}(N, \alpha)$ and the mean search time $\bar{\tau}(N, \alpha)$ vs. exponent $\alpha$ for sparse Kleinberg's network of fixed large size $N$, based on a $d$ dimensional lattice. The network with $\alpha=d$ is searchable.

(iii) for $\alpha>2, \bar{\tau}(N) \sim N^{(\alpha-2) /(2 \alpha-2)}$.

That is, $\alpha=d$ gives the best search performance (with this algorithm). In this respect, the network with $\alpha=d$ may be called "searchable" (see Fig. 42). Remarkably, $\bar{\tau}(N) \sim \bar{\ell}(N)$ in the searchable networks.

A similar phenomenon was observed also on trees with added shortcuts (Watts et al., 2002). In this situation, the probability that a shortcut connects a pair of vertices separated by $r$ steps on the tree should be taken not power-law but exponential, proportional to $\exp (-r / \xi)$. With the same greedy algorithm, using "geographic positions" of the vertices on the underlining tree, this network appears to be searchable at special values of the parameter $\xi$. Interestingly, the ferromagnetic Ising model placed on this network has long-range order only at zero temperature at any positive $\xi$ (Wołoszyn et al., 2007).

Dorogovtsev et al. (2007) exactly described a transition from a small world to a large one in growing trees with a power-law aging. Remarkably, they found that $\bar{\ell}(N) \sim \ln ^{2} N$ at the point of this transition similarly to a searchability point in Kleinberg's networks. This suggests that the tree ansatz works at a searchability point of Kleinberg's networks.

\section{G. Graph partitioning}

The size of this article does not allow us to touch upon each of studied transitions in various networks. In the end of this section, we only mention a phase transition found by Paul et al. (2007). They studied the following problem: partition a graph by removing a fraction $1-p$ of edges in a way minimizing the size of the largest partition, $S$. This problem is related to the optimal immunization strategy for a complex network. In the random regular graph with the coordination number $q$, all partitions are small if $p<p_{c}=2 / q$, where $p_{c}$ does not coincide with the usual percolation threshold, $1 /(q-1)$. On the other side of the threshold, the largest partition turns out to 
be giant, $S \sim N$. Moreover, in contrast to percolation, as the fraction $p$ of retained edges decreases, a sequence of jumps in $S$ - a sequence of "transitions" - takes place.

\section{SUMMARY AND OUTLOOK}

\section{A. Open problems}

We would like to indicate a few directions of particular interest among those discussed in this article. The first one is the synchronization in the $\mathrm{Ku}$ ramoto model on complex networks, for which there is no solid theory. The second direction is the coevolving networks and interacting systems defined on them (Holme and Newman, 2006; Pacheco et al., 2006). We did not discussed a number of interesting NP optimization problems which were studied by tools of statistical physics but were considered only for classical random graphs. Among them, there were sparse graph error correcting codes (see, e.g., Montanari (2005) and references therein), phase transitions in random satisfiability problems (Achlioptas et al., 2005; Krzakała et al., 2007; Mertens et al., 2003; (Mézard et al., 2002), and combinatorial auctions (Galla et al., 2006). Note that the coloring graph problem and minimum vertex covers were also not analysed for complex networks. Finally, we add to our list the tough but, we believe, doable problem of finding a replica-symmetry breaking solution for a spin glass on a complex network.

Real-life networks are finite, loopy (clustered) and correlated. Most of them are out of equilibrium. A solid theory of correlation phenomena in complex networks must take into account finite-size effects, loops, degree correlations and other structural peculiarities. We described two successful analytical approaches to cooperative phenomena in infinite networks. The first was based on the tree ansatz, and the second was the generalization of the Landau theory of phase transitions. What is beyond these approaches?

Several first methodical studies aiming at strict accounting for loops were performed recently, see Montanari (2005), Montanari and Rizzo (2005), and Chertkov and Chernyak (2006a, b). The approximations and loop expansions proposed in these works were not applied to complex networks yet. Rather, it is a tool for future work. It is still unknown when and how loops change cooperative phenomena in complex networks.

The tree ansatz usually fails in finite networks. In this respect, the problem of a finite size network is closely related to the problem of loops. It is technically difficult to go beyond intuitive estimates of finite-size effects demonstrated in Sec. III.B.4, and the finite-size scaling conjecture. The strict statistical mechanics theory of finite networks is still not developed.

Despite some number of interesting results, cooperative models on growing networks are poorly understood. As a rule, it is still impossible to predict the type of a critical phenomenon in an interacting system of this kind.
The effect of structural correlations in a complex network on collective phenomena is also a little studied problem.

\section{B. Conclusions}

We have reviewed recent progress in critical phenomena in complex networks. In more precise terms, we have considered critical effects in a wide range of cooperative models placed on various networks and network models. We have demonstrated a number of diverse critical effects and phenomena, which greatly differ from those in lattices. It turns out, however, that each of these phenomena in networks, in principle, can be explained in the framework of a unified approach. This unified view has been presented in this article.

We have shown that in simple terms, the brand new appearance of critical phenomena is determined by the combination of two factors - the small-world effect and a strong heterogeneity and complex architecture of networks. The compactness of networks leads to Gaussian critical fluctuations, and in this respect, the theory of phase transitions in networks is even more simple than in low-dimensional lattices. On the other hand, the complex organization of connections makes these critical phenomena far more rich and strayed from those predicted by the traditional mean-field theories.

It was claimed only four years ago that "the study of complex networks is still in its infancy" (Newman, 2003a). Now the baby has come of age. Nonetheless, we have indicated a wide circle of open problems and challenging issues. We stress that in contrast to the impressive progress in understanding the basic principles and nature of the critical phenomena in networks, progress in the application of these ideas to real-world networks is rather modest (though see, e.g., the work of Colizza et al. (2007)). There is much to be done in this direction.

Complex networks are ultimately compact, maximally disordered, and heterogeneous substrates for interacting systems. Importantly, these network systems are among the fundamental structures of nature. The phenomena and processes in these highly nontraditional systems remarkably differ from those in ordered and disordered lattices and fractals. This is why the study of these intriguing effects will lead to a new understanding of a wide circle of natural, artificial, and social systems.

\section{Acknowledgments}

We thank A. N. Samukhin, M. Alava, A.-L. Barabási, M. Bauer, O. Bénichou, G. Bianconi, M. Boguñá, B. Bollobás, S. Bornholdt, Z. Burda, S. Coulomb, D. Dhar, M. E. Fisher, M. Hase, S. Havlin, B. Kahng, T. Kaski, E. Khajeh, P. L. Krapivsky, F. Krzakala, A. Krzewicki, D. Krioukov, S. N. Majumdar, S. Maslov, D. Mukamel, M. E. J. Newman, J. D. Noh, J. G. Oliveira, M. Ostilli, J. Pacheco, H. Park, R. Pastor-Satorras, M. Peltomaki, 
A. M. Povolotsky, J. J. Ramasco, S. Redner, O. Riordan, G. J. Rodgers, M. Rosvall, B. N. Shalaev, K. Sneppen, B. Söderberg, B. Tadić, A. Vespignani, T. Vicsek, B. Waclaw, M. Weigt, L. Zdeborová, and A. Zyuzin for numerous helpful discussions and conversations on the topic of this work. We particularly thank J. G. Oliveira for numerous comments and remarks on the manuscript of this article. This work was supported by the POCI program, projects FAT/46241/2002, MAT/46176/2003, FIS/61665/2004, and BIA-BCM/62662/2004, and by the DYSONET program.

\section{APPENDIX A: BETHE-PEIERLS APPROACH: THERMODYNAMIC PARAMETERS}

The Bethe-Peierls approximation in Sec.VI.A.1 allows us to calculate a number of important thermodynamic parameters. The correlation function $C_{i j} \equiv\left\langle S_{i} S_{j}\right\rangle$ between two neighboring spins is

$$
C_{i j}=\tanh \left\{\beta J_{i j}+\tanh ^{-1}\left[\frac{\tanh \beta h_{j i} \tanh \beta h_{i j}}{\tanh ^{2} \beta J_{i j}}\right]\right\} .
$$

Notice that $C_{i j}$ is completely determined by the messages $h_{i j}$ and $h_{j i}$ which two neighboring spins, $i$ and $j$, send to each other. Knowing $C_{i j}$ and $M_{i}$, we find the internal energy

$$
E=-\sum_{(i j)} J_{i j} a_{i j} C_{i j}-\sum_{i} H_{i} M_{i}
$$

and the free energy (Mézard and Parisi, 2001)

$$
F=\sum_{(i j)} F_{(i j)}^{(2)}-\sum_{i}\left(q_{i}-1\right) F_{i}^{(1)}
$$

where

$$
\begin{aligned}
F_{i}^{(1)}= & -T \ln \left\{\sum_{S_{i}= \pm 1} \exp \left[\beta\left(H_{i}+\sum_{j \in N(i)} h_{j i}\right) S_{i}\right]\right\}, \\
F_{(i j)}^{(2)}= & -T \ln \left\{\sum _ { S _ { i } , S _ { j } = \pm 1 } \operatorname { e x p } \left[\beta J_{i j} S_{i} S_{j}+\beta \varphi_{i \backslash j} S_{i}\right.\right. \\
& \left.\left.+\beta \varphi_{j \backslash i} S_{j}\right]\right\}
\end{aligned}
$$

The free energy $F$ satisfies the thermodynamic relations: $\partial(\beta F) / \partial \beta=E, \partial F / \partial H_{i}=-M_{i}$, and the extremum condition $\partial F / \partial h_{j i}=0$.

\section{APPENDIX B: BELIEF-PROPAGATION ALGORITHM: MAGNETIC MOMENT AND THE BETHE FREE ENERGY}

Using the belief-propagation algorithm discussed in Sec.VI.A.2 we can easily calculate a local magnetic moment:

$$
M_{i}=\sum_{S_{i}= \pm 1} S_{i} b_{i}\left(S_{i}\right)
$$

a)

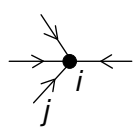

b)

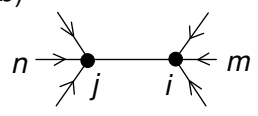

FIG. 43 Diagram representation of the beliefs (a) $b_{i}$ and (b) $b_{j i}$. Notations are explained in Fig. 21

where $b_{i}\left(S_{i}\right)$ is the probability of finding a spin $i$ in state $S_{i}$. This probability is normalized, $\sum_{S_{i}= \pm 1} b_{i}\left(S_{i}\right)=1$, and related to the fixed point probabilities $\left\{\mu_{j i}\left(S_{i}\right)\right\}$ and the probabilistic factor $\exp \left(\beta H_{i} S_{i}\right)$, see Fig. 43 .

$$
b_{i}\left(S_{i}\right)=A e^{\beta H_{i} S_{i}} \prod_{j \in N(i)} \mu_{j i}\left(S_{i}\right)
$$

where $A$ is a normalization constant.

The correlation function $C_{i j}=\left\langle S_{i} S_{j}\right\rangle$ is determined by the probability $b_{i j}\left(S_{i}, S_{j}\right)$ to simultaneously find neighboring spins $i$ and $j$ in spin states $S_{i}$ and $S_{j}$ :

$$
C_{i j}=\sum_{S_{i}, S_{j}= \pm 1} S_{i} S_{j} b_{i j}\left(S_{i}, S_{j}\right)
$$

where

$$
\sum_{S_{j}= \pm 1} b_{i j}\left(S_{i}, S_{j}\right)=b_{i}\left(S_{i}\right)
$$

In the belief-propagation algorithm, the probabilities $b_{i}$ and $b_{i j}$ are called "beliefs". Using Fig. 43, we obtain

$$
\begin{aligned}
b_{i j}\left(S_{i}, S_{j}\right)= & A e^{\beta H_{i} S_{i}+\beta J_{i j} S_{i} S_{j}+\beta H_{j} S_{j}} \\
& \times \prod_{n \in N(j) \backslash i} \mu_{n j}\left(S_{j}\right) \prod_{n \in N(i) \backslash j} \mu_{m i}\left(S_{i}\right) .
\end{aligned}
$$

At the fixed point, we find that $C_{i j}$ is given by Eq. (A1).

Yedidia et al. (2001) proved that at the fixed point the beliefs $\left\{b_{i}, b_{i j}\right\}$ give a local minimum of the Bethe free energy $F_{\mathrm{B}}$ :

$$
\begin{aligned}
F_{\mathrm{B}}\left(\left\{b_{i}, b_{i j}\right\}\right)= & \sum_{(i j)} \sum_{S_{i}, S_{j}= \pm 1} b_{i j}\left(S_{i}, S_{j}\right) \ln \frac{b_{i j}\left(S_{i}, S_{j}\right)}{\phi_{i j}\left(S_{i}, S_{j}\right)} \\
& -\sum_{i}\left(q_{i}-1\right) \sum_{S_{i}= \pm 1} b_{i}\left(S_{i}\right) \ln \frac{b_{i}\left(S_{i}\right)}{\psi_{i}\left(S_{i}\right)},
\end{aligned}
$$

where $\psi_{i}\left(S_{i}\right)=\exp \left(\beta H_{i} S_{i}\right), \phi_{i j}\left(S_{i}, S_{j}\right)=\exp \left(\beta H_{i} S_{i}+\right.$ $\left.\beta J_{i j} S_{i} S_{j}+\beta H_{j} S_{j}\right)$.

\section{APPENDIX C: REPLICA TRICK}

The replica trick is a powerful mathematical method which allows one to average over a quenched disorder. We first introduce a statistical network ensemble, describe an average over a network ensemble, and then develop a 
replica approach for the ferromagnetic Ising model on an uncorrelated random network.

Let us consider the Erdős-Rényi graph of $N$ vertices. The probability $P_{a}\left(a_{i j}\right)$ that an edge between vertices $i$ and $j$ is present $\left(a_{i j}=1\right)$ or absent $\left(a_{i j}=0\right)$ is

$$
P_{a}\left(a_{i j}\right)=\frac{z_{1}}{N} \delta\left(a_{i j}-1\right)+\left(1-\frac{z_{1}}{N}\right) \delta\left(a_{i j}\right)
$$

where $a_{i j}$ are the adjacency matrix elements, $z_{1} \equiv\langle q\rangle$ is average degree. Given that the matrix elements are independent and uncorrelated random parameters, the probability of realization of a graph with a given adjacency matrix $a_{i j}$, is the product of probabilities $P_{a}\left(a_{i j}\right)$ over all pairs of vertices:

$$
\mathcal{P}\left(\left\{a_{i j}\right\}\right)=\prod_{i=1}^{N-1} \prod_{j=i+1}^{N} P_{a}\left(a_{i j}\right)
$$

The average of a physical quantity $A\left(\left\{a_{i j}\right\}\right)$ over the network ensemble is

$$
\langle A\rangle_{\mathrm{en}}=\int A\left(\left\{a_{i j}\right\}\right) \mathcal{P}\left(\left\{a_{i j}\right\}\right) \prod_{i=1}^{N-1} \prod_{j=i+1}^{N} d a_{i j}
$$

In the configuration model with a given degree distribution $P(q)$ the probability of the realization of a given graph is

$$
\mathcal{P}\left(\left\{a_{i j}\right\}\right)=\frac{1}{\mathcal{N}} \prod_{i=1}^{N-1} \prod_{j=i+1}^{N} P_{a}\left(a_{i j}\right) \prod_{i=1}^{N} \delta\left(\sum_{j} a_{i j}-q_{i}\right) .
$$

The delta-functions fix degrees of the vertices. $\mathcal{N}$ is a normalization factor:

$$
\mathcal{N}=\exp \left[N \sum_{q} P(q) \ln \left(z_{1}^{q} / q !\right)-N z_{1}\right] .
$$

$P_{a}\left(a_{i j}\right)$ is given by the same Eq. (C1).

In the static model of a complex network (see Sec. II.E.2), a desired degree $d_{i}$ is assigned to each vertex $i$. The probability that vertices $i$ and $j$ are linked is equal to $p_{i j}$. With the probability $\left(1-p_{i j}\right)$ the edge $(i j)$ is absent. We have

$$
P_{a}\left(a_{i j}\right)=p_{i j} \delta\left(a_{i j}-1\right)+\left(1-p_{i j}\right) \delta\left(a_{i j}\right),
$$

where $p_{i j}=1-\exp \left(-N d_{i} d_{j} / N\langle d\rangle\right)$. The probability $\mathcal{P}\left(\left\{a_{i j}\right\}\right)$ is given by Eq. (C2).

The replica trick is usually used for calculating an average free energy $\langle F\rangle_{\mathrm{av}}=-\langle T \ln Z\rangle_{\mathrm{av}}$, where $\langle\ldots\rangle_{\mathrm{av}}$ is an average over a quenched disorder. The replica trick is based on the identity:

$$
\langle\ln Z\rangle_{\mathrm{av}}=\lim _{n \rightarrow 0} \frac{\left\langle Z^{n}\right\rangle_{\mathrm{av}}-1}{n}=\lim _{n \rightarrow 0} \frac{\ln \left\langle Z^{n}\right\rangle_{\mathrm{av}}}{n} .
$$

Let us demonstrate averaging over the statistical ensemble Eq. (C4) for the configuration model:

$$
\left\langle Z^{n}\right\rangle_{\mathrm{en}}=\int Z^{n} \prod_{i=1}^{N} \delta\left(\sum_{j} a_{i j}-q_{i}\right) \prod_{i=1}^{N-1} \prod_{j=i+1}^{N} P_{a}\left(a_{i j}\right) d a_{i j} .
$$

We consider the ferromagnetic Ising model with $J_{i j}=J$ in a uniform field $H$, placed on the configuration model (Leone et al., 2002). Using an integral representation of the constrains

$$
\delta\left(\sum_{j} a_{i j}-q_{i}\right)=\int_{-\infty}^{\infty} \frac{d \psi_{i}}{2 \pi} e^{i\left(\sum_{j} a_{i j}-q_{i}\right) \psi_{i}},
$$

we integrate over $a_{i j}$ with the probability function $P_{a}\left(a_{i j}\right)$ given by Eq. (C1):

$$
\begin{aligned}
& \int \exp \left[\beta J a_{i j} \mathbf{S}_{i} \mathbf{S}_{j}+i a_{i j}\left(\psi_{i}+\psi_{j}\right)\right] P_{a}\left(a_{i j}\right) d a_{i j} \\
& =1+\frac{z_{1}}{N}\left(e^{\beta J \mathbf{S}_{i} \mathbf{S}_{j}+i\left(\psi_{i}+\psi_{j}\right)}-1\right) \\
& \approx \exp \left[\frac{z_{1}}{N}\left(e^{\beta J \mathbf{S}_{i} \mathbf{S}_{j}+i\left(\psi_{i}+\psi_{j}\right)}-1\right)\right],
\end{aligned}
$$

where $\mathbf{S}_{i} \equiv\left(S_{i}^{1}, S_{i}^{2}, \ldots, S_{i}^{n}\right), \mathbf{S}_{i} \mathbf{S}_{j} \equiv \sum_{\alpha} S_{i}^{\alpha} S_{j}^{\alpha} . \quad \alpha=$ $1,2, \ldots, n$ is the replica index. Note that one can simultaneously integrate over random couplings $J_{i j}$ and random fields $H_{i}$.

In the limit $N \gg 1$ we obtain

$$
\begin{array}{r}
\left\langle Z^{n}\right\rangle_{\mathrm{en}}=\frac{1}{\mathcal{N}} \sum_{\left\{S_{i}^{\alpha}= \pm 1\right\}} \int\left(\prod_{i} \frac{d \psi_{i}}{2 \pi} e^{-i q_{i} \psi_{i}}\right) \times \\
\exp \left[\frac{z_{1}}{2 N} \sum_{i j} e^{\beta J \mathbf{S}_{i} \mathbf{S}_{j}+i\left(\psi_{i}+\psi_{j}\right)}+\beta \sum_{i} \mathbf{H S}_{i}-\frac{1}{2} N z_{1}\right],(\mathrm{C} 1)
\end{array}
$$

where $\mathbf{H S}=\sum_{\alpha} H S^{\alpha}$.

Let us introduce a functional order parameter:

$$
\rho(\sigma)=\frac{1}{N} \sum_{i} \delta\left(\sigma-\mathbf{S}_{i}\right) e^{i \psi_{i}}
$$

where $\sigma=\left(\sigma^{1}, \sigma^{2}, \ldots, \sigma^{n}\right)$. There is an identity:

$$
\frac{1}{N} \sum_{i j} e^{\beta J \mathbf{S}_{i} \mathbf{S}_{j}+i\left(\psi_{i}+\psi_{j}\right)}=N \sum_{\sigma_{1}, \sigma_{2}} \rho\left(\sigma_{1}\right) \rho\left(\sigma_{2}\right) e^{\beta J \sigma_{1} \sigma_{2}}
$$

We use the functional Hubbard-Stratonovich transformation:

$$
\begin{aligned}
& \exp \left[\frac{N z_{1}}{2} \sum_{\left\{\sigma_{1}, \sigma_{2}= \pm 1\right\}} \rho\left(\sigma_{1}\right) \rho\left(\sigma_{2}\right) e^{\beta J \sigma_{1} \sigma_{2}}\right] \\
& =\int D \widehat{\rho}(\sigma) \exp \left[-\frac{N z_{1}}{2} \sum_{\sigma_{1}, \sigma_{2}} \widehat{\rho}\left(\sigma_{1}\right) C\left(\sigma_{1}, \sigma_{2}\right) \widehat{\rho}\left(\sigma_{2}\right)\right. \\
& \left.\quad+N z_{1} \sum_{\sigma} \widehat{\rho}(\sigma) \rho(\sigma)\right] .
\end{aligned}
$$

Here $C\left(\sigma_{1}, \sigma_{2}\right)$ is an inverse function to $e^{\beta J \sigma_{1} \sigma_{2}}$ :

$$
\sum_{\sigma_{1}} C\left(\sigma, \sigma_{1}\right) e^{\beta J \sigma_{1} \sigma_{2}}=\delta\left(\sigma-\sigma_{2}\right) .
$$


The transformation Eq. C14 enables us to integrate over variables $\psi_{i}$ in Eq. (C11):

$$
\begin{gathered}
\left\langle Z^{n}\right\rangle_{\mathrm{en}}=\int \frac{D \widehat{\rho}(\sigma)}{\mathcal{N}} \exp \left\{-\frac{N z_{1}}{2} \sum_{\sigma_{1}, \sigma_{2}} \widehat{\rho}\left(\sigma_{1}\right) C\left(\sigma_{1}, \sigma_{2}\right) \widehat{\rho}\left(\sigma_{2}\right)\right. \\
\left.+N \sum_{q} P(q) \ln \left[\sum_{\mathbf{S}} \frac{1}{q !} z_{1}^{q} \widehat{\rho}^{q}(\mathbf{S}) e^{\beta \mathbf{H S}}\right]-\frac{1}{2} N z_{1}\right\} \cdot(\mathrm{C} 16)
\end{gathered}
$$

In the thermodynamic limit $N \rightarrow \infty$, the functional integral over $\widehat{\rho}(\sigma)$ is calculated by using the saddle point method. The saddle point equations are

$$
\begin{aligned}
& \widehat{\rho}(\mathbf{S})=\sum_{\sigma} \rho(\sigma) e^{\beta J \sigma \mathbf{S}}, \\
& \rho(\mathbf{S})=\sum_{q} \frac{P(q) q}{z_{1}} \frac{\widehat{\rho}^{q-1}(\mathbf{S}) e^{\beta \mathbf{H S}}}{\sum_{\mathbf{S}} \widehat{\rho}^{q}(\mathbf{S}) e^{\beta \mathbf{H S}}} .
\end{aligned}
$$

Equation (C16) gives the replica free energy per vertex:

$$
\begin{aligned}
-n \beta F N^{-1}= & N^{-1} \ln \left\langle Z^{n}\right\rangle_{\mathrm{av}}=-z_{1} \sum_{\sigma} \widehat{\rho}(\sigma) \rho(\sigma)+\frac{1}{2} z_{1} \\
& +\frac{z_{1}}{2} \sum_{\sigma_{1}, \sigma_{2}} \rho\left(\sigma_{1}\right) \rho\left(\sigma_{2}\right) e^{\beta J \sigma_{1} \sigma_{2}} \\
& +\sum_{q} P(q) \ln \left[\sum_{\mathbf{S}} \widehat{\rho}^{q}(\mathbf{S}) e^{\beta \mathbf{H S}}\right] . \quad(\mathrm{C} 19)
\end{aligned}
$$

A replica symmetric solution of the saddle-point equations (C17) and (C18) can be written in a general form:

$$
\begin{aligned}
& \rho(\mathbf{S})=\int d h \Phi(h) \frac{e^{\beta \mathbf{h S}}}{(2 \cosh \beta h)^{n}}, \\
& \widehat{\rho}(\mathbf{S})=\int d h \Psi(h) \frac{e^{\beta \mathbf{h S}}}{(2 \cosh \beta h)^{n}},
\end{aligned}
$$

where $\mathbf{h S}=h \sum_{\alpha=1}^{n} S^{\alpha}$. Substituting the replica symmetric solution into the saddle point equations (C17) and (C18), we obtain

$$
\begin{aligned}
& \Phi(h)=\sum_{q} \frac{P(q) q}{z_{1}} \int \delta\left(h-\sum_{m=1}^{q-1} h_{m}-H\right) \prod_{m=1}^{q-1} \Psi\left(h_{m}\right) d h_{m}, \\
& \Psi(h)=\int \delta\left(h-T \tanh ^{-1}[\tanh \beta J \tanh \beta y]\right) \Phi(y) d y \cdot(\mathrm{C} 22
\end{aligned}
$$

Substituting $\Phi(h)$ into the equation for $\Psi(h)$, we obtain the self-consistent equation (82) derived by using the Bethe-Peierls approximation. $\Psi(h)$ is actually the distribution function of additional fields (messages) in a network.

\section{APPENDIX D: MAX-CUT ON THE ERDÖS-RÉNYI GRAPH}

One can prove the validity of the upper bound Eq. (99) for the maximum cut $K_{c}$ on the Erdős-Rényi graph, using the so called first-moment method.
We divide the Erdős-Rényi graph into two sets of $S$ and $N-S$ vertices. The probability that a randomly chosen edge has endpoints from different sets is $Q=2(S / N)(1-$ $S / N)$. The number of ways to divide a graph by a cut of $K$ edges is

$$
\mathfrak{N}(K)=\left(\begin{array}{c}
L \\
K
\end{array}\right) \sum_{S=0}^{N}\left(\begin{array}{c}
N \\
S
\end{array}\right) Q^{K}(1-Q)^{L-K} .
$$

Here $\left(\begin{array}{c}L \\ K\end{array}\right)$ is the number of ways to choose $K$ edges from $L$ edges, $\left(\begin{array}{l}N \\ S\end{array}\right)$ is the number of ways to choose $S$ vertices from $N$ vertices, $Q^{K}(1-Q)^{L-K}$ is the probability that there are $K$ edges in the cut, and the $L-K$ remaining edges do not belong to the cut. The main contribution to $\mathfrak{N}(K)$ is given by terms with $S \approx N / 2$, i.e., $Q \approx 1 / 2$. So,

$$
\mathfrak{N}(K) \approx\left(\begin{array}{c}
L \\
K
\end{array}\right) 2^{N-L}=e^{L \Xi(\alpha)},
$$

where $\alpha=K / L$. Using the entropy bound on the binomial,

$$
\left(\begin{array}{l}
L \\
K
\end{array}\right) \leqslant \exp [-L(1-\alpha) \ln (1-\alpha)-L \alpha \ln \alpha],
$$

we find

$$
\Xi(\alpha)=-(1-\alpha) \ln (1-\alpha)-\alpha \ln \alpha+\left(z_{1} / 2-1\right) \ln 2 .
$$

The maximum cut $K_{c}$ is given by the condition $\Xi\left(\alpha_{c}\right)=$ 0 . In the limit $N \rightarrow \infty$, there is no cut with a size $K>\alpha_{c} L=K_{c}$ while there are exponentially many cuts at $K<K_{c}$. This condition at $z_{1} \gg 1$ leads to Eq. (99) with the upper bound $A=\sqrt{\ln 2} / 2$.

\section{APPENDIX E: EQUATIONS OF STATE OF THE POTTS MODEL ON A NETWORK}

Dorogovtsev et al. (2004) showed that for the ferromagnetic $p$-state Potts model Eq. (119) on an uncorrelated random graph, the magnetic moments $M_{i} \equiv M_{i}^{(1)}$ along the magnetic field $H$ and the additional fields (messages) $h_{i j}$ are determined by the following equations:

$$
\begin{aligned}
M_{i} & =\frac{1-\exp \left[-\beta\left(H+\sum_{j \in N(i)} h_{j i}\right)\right]}{1+(p-1) \exp \left[-\beta\left(H+\sum_{j \in N(i)} h_{j i}\right)\right]}, \\
h_{i j} & =T \ln \left\{\frac{e^{\beta J_{i j}}+(p-1) e^{-\beta \varphi_{i \backslash j}}}{1+\left(e^{\beta J_{i j}}+p-2\right) e^{-\beta \varphi_{i \backslash j}}}\right\},
\end{aligned}
$$

where $\varphi_{i \backslash j}=H+\sum_{m \in N(i) \backslash j} h_{m i}$ is the cavity field. These equations unify the percolation, the ferromagnetic Ising model and a first order phase transition on uncorrelated complex networks. They are exact in the limit $N \rightarrow \infty$. 
For the one-state ferromagnetic Potts model in zero field, Eq. (E2) takes a simple form:

$$
x_{i j}=1-r+r \prod_{m \in N(i) \backslash j} x_{m i},
$$

where $x_{i j} \equiv \exp \left(-h_{i j}\right)$, the coupling $J_{i j}=J>0$, and $r \equiv\left(1-e^{-\beta J}\right)$. In the configuration model, the parameters $x_{m i}$ are statistically independent. Averaging over the network ensemble and introducing the parameter $x \equiv\left\langle x_{i j}\right\rangle_{\text {en }}$, we arrive at Eq. (14) describing bond percolation on uncorrelated networks. The critical temperature $T_{\mathrm{P}}$ in Eq. (121) determines the percolation threshold $r\left(T=T_{\mathrm{P}}\right)=z_{1} / z_{2}$ in agreement with Eq. (17).

When $p=2$, equation (E2) is reduced to Eq. (60) for the Ising model. It is only necessary to rescale $J \rightarrow 2 J$, $H \rightarrow 2 H$, and $h \rightarrow 2 h$.

\section{References}

Acebrón, J. A., L. L. Bonilla, C. J. Pérez Vicente, F. Ritort, and R. Spigler, 2005, "The Kuramoto model: A simple paradigm for synchronization phenomena," Rev. Mod. Phys. 77, 137.

Achlioptas, D., A. Naor, and Y. Peres, 2005, "Rigorous location of phase transitions in hard optimization problems," Nature 435, 759 .

Adamic, L. A., R. M. Lukose, and B. . Huberman, 2003, "Local search in unstructured networks," in Handbook of Graphs and Networks, edited by S. Bornholdt and H. G. Schuster (Wiley-VCH GmbH \& Co., Weinheim), p. 295.

Aharony, A. 1978, "Tricritical points in systems with random fieldsl," Phys. Rev. B 18, 3318.

Aizenman, M. and J. L. Lebowitz, 1988, "Metastability effects in bootstrap percolation," J. Phys. A 21, 3801.

Alava, M. J. and S. N. Dorogovtsev, 2005, "Complex networks created by aggregation," Phys. Rev. E 71, 036107.

Albert, R. and A.-L. Barabási, 2002, "Statistical mechanics of complex networks," Rev. Mod. Phys. 74, 47.

Albert, R., H. Jeong, and A.-L. Barabási, 2000, "Error and attack tolerance in complex networks," Nature 406, 378.

Albert, R., H. Jeong, and A.-L. Barabási, 1999, "Diameter of the world-wide web," Nature 401, 130.

Aleksiejuk, A., J. A. Holyst, and D. Stauffer, 2002, "Ferromagnetic phase transition in Barabási-Albert networks," Physica A 310, 260.

Allahverdyan, A. E. and K. G. Petrosyan, 2006, "Statistical networks emerging from link-node interactions," Europhys. Lett. 75, 908.

Alvarez-Hamelin, J. I., L. Dall'Asta, A. Barrat, and A. Vespignani, 2006, " $k$-core decomposition: a tool for the visualization of large scale networks," Advances in Neural Information Processing Systems (Canada) 18, 41.

Alvarez-Hamelin, J. I., L. Dall'Asta, A. Barrat, and A. Vespignani, 2005b, " $k$-core decomposition: a tool for the analysis of large scale Internet graphs," eprint cs.NI/0511007.

Andrade, R. F. S. and H. J. Herrmann, 2005, "Magnetic models on Apollonian networks," Phys. Rev. E 71, 056131.
Andrade Jr., J. S., H. J. Herrmann, R. F. S. Andrade, and L. R. da Silva, 2005, "Apollonian networks," Phys. Rev. Lett. 94, 018702.

Angel, A. G., M. R. Evans, E. Levine, and D. Mukamel, 2005, "Critical phase in nonconserving zero-range process and rewiring networks," Phys. Rev. E 72, 046132.

Angel, A. G., T. Hanney, and M. R. Evans, 2006, "Condensation transitions in a model for a directed network with weighted links," Phys. Rev. E 73, 016105.

Antal, T., P. L. Krapivsky, and S. Redner, 2005, "Dynamics of social balance on networks," Phys. Rev. E 72, 036121.

Antoni, M. and S. Ruffo, 1995, "Clustering and relaxation in Hamiltonian long-range dynamics," Phys. Rev. E 52, 2361.

Appel, K. and W. Haken, 1977a, "Every planar map is four colorable. Part I. Discharging," Illinois J. Math. 21, 429.

Appel, K. and W. Haken, 1977b, "Every planar map is four colorable. Part II. Reducibility," Illinois J. Math. 21, 491.

de Arcangelis, L. and H. Herrmann, 2002, "Self-organized criticality on small world networks," Physica A 308, 545.

Arenas, A., A. Díaz-Guilera, and R. Guimera, 2001, "Communication in networks with hierarchical branching," Phys. Rev. Lett. 86, 3196.

Arenas A., A. Díaz-Guilera, and C. J. Pérez-Vicente, 2006a, "Synchronization reveals topological scales in complex networks," Phys. Rev. Lett. 96, 114102.

Arenas, A., A. Díaz-Guilera, and C. J. Perez-Vicente, 2006b, "Synchronization processes in complex networks," Physica D 224, 27.

Aronson, J., A., Frieze, and B. G., Pittel, 1998, "Maximum matchings is sparse random graphs: Karp-Sipser revisited," Rand. Struct. Algor. 12, 111.

Atay, F. M., J. Jost, and A. Wende, 2004, "Delays, Connection Topology, and Synchronization of Coupled Chaotic Maps," Phys. Rev. Lett. 92, 144101.

ben-Avraham, D. and M. L. Glasser, 2007, "Diffusion-limited one-species reactions in the Bethe lattice," J. Phys. C 19, 065107.

Baillie, C., W. Janke, D. Johnston, and P. Plecháč, 1995, "Spin glasses on thin graphs," Nucl. Phys. B 450, 730.

Bakke, J. O. H., A. Hansen, and J. Kertész, 2006, "Failure and avalanches in complex networks," Europhys. Lett. 76, 717.

Barabási, A.-L. and R. Albert, 1999, "Emergence of scaling in complex networks," Science 286, 509.

Barabási, A.-L., E. Ravasz, and T. Vicsek, 2001, "Deterministic scale-free networks," Physica A 299, 559.

Barahona, M. and L. Pecora, 2002, "Synchronization in smallworld systems," Phys. Rev. Lett. 89, 054101.

Baronchelli, A., L. Dall'Asta, A. Barrat, and V. Loreto, 2007, "Non-equilibrium phase transition in negotiation dynamics," Phys. Rev. E 76, 051102.

Barrat, A. and M. Weigt, 2000, "On the properties of smallworld models," Eur. Phys. J. B 13, 547.

Barthel, W. and A. K. Hartmann, 2004, "Clustering analysis of the ground-state structure of the vertex-cover problem," Phys. Rev. E 70, 066120.

Barthelemy, M., A. Barrat, R. Pastor-Satorras, and A. Vespignani, 2004, "Velocity and hierarchical spread of epidemic outbreaks in scale-free networks," Phys. Rev. Lett. 92, 178701 .

Barthelemy, M., A. Barrat, R. Pastor-Satorras, and A. Vespignani, 2005, "Dynamical patterns of epidemic outbreaks in complex heterogeneous networks," J. Theor. Biology 235, 275. 
Bartolozzi, M., T. Surungan, D. B. Leinweber, and A. G. Williams, 2006, "Spin glass behavior of the antiferromagnetic Ising model on a scale-free network," Phys. Rev. B 73, 224419.

Bauer, M. and D. Bernard, 2002, "Maximal entropy random networks with given degree distribution," eprint condmat/0206150.

Bauer, M., S. Coulomb, and S. N. Dorogovtsev, 2005, "Phase transition with the Berezinskii-Kosterlitz-Thouless singularity in the Ising model on a growing network," Phys. Rev. Lett. 94, 200602.

Bauer, M. and O. Golinelli, 2001a, "Core percolation in random graphs: a critical phenomena analysis," Eur Phys. J. B 24, 339.

Bauer, M. and O. Golinelli, 2001b, "Exactly solvable model with two conductor-insulator transitions driven by impurities," Phys. Rev. Lett. 86, 2621.

Baxter, R. J., 1982, Exactly Solved Models in Statistical Mechanics (Academic Press, London).

Bedeaux, D., K. E. Shuler, and I. Oppenheim, 1970, "Decay of correlations. III. Relaxation of spin correlations and distribution functions in the one-dimensional Ising lattice," J. Stat. Phys. 2, 1.

ben-Avraham, D., D. B. Considine, P. Meakin, S. Redner, and H. Takayasu, 1990, "Saturation transition in a monomermonomer model of heterogeneous catalysis," J. Phys. A 23, 4297.

Ben-Naim, E., and P. L. Krapivsky, 2004, "Size of outbreaks near the epidemic threshold," Phys. Rev. E 69, 050901 (R).

Bender, E. A. and E. R. Canfield, 1978, "The asymptotic number of labeled graphs with given degree sequences," J. Combin. Theor. A 24, 296.

Bénichou, O. and R. Voituriez, 2007, "Comment on 'Localization Transition of Biased Random Walks on Random Networks'," Phys. Rev. Lett. 99, 209801.

Benjamini, I. and N. Berger, 2001, "The diameter of longrange percolation clusters on finite cycles," Rand. Struct. Algor. 19, 102.

Berezinskii, V. L., 1970, "Destruction of long range order in one-dimensional and twodimensional systems having a continuous symmetry group: I. Classical systems," ZhETF 59, 907 [Sov. Phys. JETP 32, 493].

Berg, J. and M. Lässig, 2002, "Correlated random networks," Phys. Rev. Lett. 89, 228701.

Bernardo, M., F. Garofalo, and F. Sorrentino, 2007, "Synchronizability and synchronization dynamics of weighed and unweighed scale free networks with degree mixing," Int. J. Bifurcation and Chaos 17, 7.

Bethe, H. A., 1935, "Statistical theory of superlattices," Proc. Roy. Soc. London A 150, 552.

Bialas, P., L. Bogacz, B. Burda, and D. Johnston, 2000, "Finite size scaling of the balls in boxes model," Nucl. Phys. B 575, 599 .

Bialas, P., Z. Burda, and D. Johnston, 1997, "Condensation in the backgammon model," Nucl. Phys. B 493, 505.

Bialas, P., Z. Burda, J. Jurkiewicz, and A. Krzywicki, 2003, "Tree networks with causal structure," Phys. Rev. E 67, 066106.

Bianconi, G., 2002, "Mean field solution of the Ising model on a Barabási-Albert network," Phys. Lett. A 303, 166.

Bianconi, G. and A.-L. Barabási, 2001, "Bose-Einstein condensation in complex networks," Phys. Rev. Lett. 86, 5632.

Bianconi, G. and A. Capocci, 2003, "Number of loops of size $h$ in growing scale-free networks," Phys. Rev. Lett. 90,
078701.

Bianconi, G., N. Gulbahce, and A. E. Motter, 2007, "Local structure of directed networks," eprint arXiv:0707.4084 [physics].

Bianconi, G. and M. Marsili, 2005a, "Loops of any size and Hamilton cycles in random scale-free networks," J. Stat. Mech. P06005.

Biely, C., R. Hanel, and S. Thurner, 2007, "Solvable spin model on dynamical networks," eprint arXiv:0707.3085 [cond-mat].

Binder, K. and A. P. Young, 1986, "Spin glasses: Experimental facts, theoretical concepts, and open questions," Rev. Mod. Phys. 58, 801.

Biskup, M., 2004, "On the scaling of the chemical distance in long-range percolation models," Ann. Probab. 32, 2938.

Blatt, M., S. Wiseman, and E. Domany, 1996, "Superparamagnetic clustering of data," Phys. Rev. Lett. 76, 3251.

Boccaletti, S., V. Latora, Y. Moreno, M. Chavez, and D.U. Hwang, 2006, "Complex networks: Structure and dynamics," Phys. Rep. 424, 175.

Bogacz, L., Z. Burda, W. Janke, and B. Waclaw, 2007a, "Balls-in-boxes condensation on networks," Chaos 17, 026112.

Bogacz, L., Z. Burda, W. Janke, and B. Waclaw, 2007b, "Free zero-range processes on networks," eprint arXiv:0705.0549 [cond-mat].

Boguñá, M. and R. Pastor-Satorras, 2002, "Epidemic spreading in correlated complex networks," Phys. Rev. E 66, 047104 .

Boguñá, M. and R. Pastor-Satorras, 2003, "Class of correlated random networks with hidden variables," Phys. Rev. E 68, 036112.

Boguñá, M., R. Pastor-Satorras, and A. Vespignani, 2003a, "Absence of epidemic threshold in scale-free networks with degree correlations ," Phys. Rev. Lett. 90, 028701.

Boguñá, M., R. Pastor-Satorras, and A. Vespignani, 2003b, "Epidemic spreading in complex networks with degree correlations," Lect. Notes Phys. 625, 127.

Boguñá, M., R. Pastor-Satorras, and A. Vespignani, 2004, "Cut-offs and finite size effects in scale-free networks," Eur. Phys. J. B 38, 205.

Boguñá, M., and M. A. Serrano, 2005, "Generalized percolation in random directed networks," Phys. Rev. E 72, 016106.

Bollobás, B., 1980, "A probabilistic proof of an asymptotic formula for the number of labelled regular graphs," Eur. J. Comb. 1, 311.

Bollobás, B., 1984, "The evolution of sparse graphs," in Graph Theory and Combinatorics: Proc. Cambridge Combinatorial Conf. in honour of Paul Erdős, edited by B. Bollobás (Academic Press, New York), p. 35.

Bollobás, B. and O. Riordan, 2003, "Mathematical results on scale-free graphs," in Handbook of Graphs and Networks, edited by S. Bornholdt and H. G. Schuster (Wiley-VCH GmbH \& Co., Weinheim), p. 1.

Bollobás, B. and O. Riordan, 2005, "Slow emergence of the giant component in the growing $m$-out graph," Random Struct. Algor. 27, 1.

Bonabeau E., 1995, "Sandpile dynamics on random graphs," J. Phys. Soc. Japan 64, 327.

Borgs, C., J. T. Chayes, H. Kesten, and J. Spencer, 2001, "The birth of the infinite cluster: Finite-size scaling in percolation," Comm. Math. Phys. 224, 153.

Brandes, U., D. Delling, M. Gaertler, R. Görke, M. Hoefer, 
Z. Nikoloski, and D. Wagner, 2006, "Maximizing Modularity is hard," eprint arXive:physics/0608255.

Braunstein, L. A., S. V. Buldyrev, R. Cohen, S. Havlin, and H. E. Stanley, 2003a, "Optimal paths in disordered complex networks," Phys. Rev. Lett. 91, 168701.

Braunstein, A., R. Mulet, A. Pagnani, M. Weigt, and R. Zecchina, 2003b, "Polynomial iterative algorithms for coloring and analyzing random graphs," Phys. Rev. E 68, 036702 .

Braunstein L. A., S. V. Buldyrev, S. Sreenivasan, R. Cohen, S. Havlin, and H. E. Stanley, 2004, "The optimal path in a random network," Lecture Notes in Physics 650, 127.

Braunstein, A. and R. Zecchina, 2004, "Survey propagation as local equilibrium equations," J. Stat. Mech. P06007.

Bruinsma, R. 1984, "Random-field Ising model on a Bethe lattice," Phys. Rev. B 30, 289.

Burda, Z., J. D. Correia, and A. Krzywicki, 2001, "Statistical ensemble of scale-free random graphs," Phys. Rev. E 64, 046118.

Burda, Z., D. Johnston, J. Jurkiewicz, M. Kaminski, M. A. Nowak, and G. Papp, 2002, "Wealth condensation in Pareto macro-economies," Phys. Rev. E 65, 026102.

Burda, Z., J. Jurkiewicz, and A. Krzywicki, 2004a, "Network transitivity and matrix models," Phys. Rev. E 69, 026106.

Burda, B., J. Jurkiewicz, and A. Krzywicki, 2004b, "Perturbing general uncorrelated networks," Phys. Rev. E $\mathbf{7 0}$, 026106.

Burda, Z. and A. Krzywicki, 2003, "Uncorrelated random networks," Phys. Rev. E 6, 046118.

Caldarelli, G., 2007, Scale-Free Networks: Complex Webs in Nature and Technology (Oxford Finance Series-Oxford University Press, Oxford).

Caldarelli, G., A. Capocci, and D. Garlaschelli, 2006, "Selforganized network evolution coupled to extremal dynamics," eprint cond-mat/0611201.

Caldarelli, G., A. Capocci, P. De Los Rios, and M. A. Muñoz, 2002, "Scale-free networks from varying vertex intrinsic fitness," Phys. Rev. Lett. 89, 258702.

Callaway, D. S., J. E. Hopcroft, J. M. Kleinberg, M. E. J. Newman, and S. H. Strogatz, 2001, "Are randomly grown graphs really random?," Phys. Rev. E 64, 041902.

Callaway, D. S., M. E. J. Newman, S. H. Strogatz, and D. J. Watts, 2000, "Network robustness and fragility: Percolation on random graphs," Phys. Rev. Lett. 85, 5468.

Carmi, S., R. Cohen, and D. Dolev, 2006a, "Searching complex networks efficiently with minimal information," Europhys. Lett. 74, 1102.

Carmi, S., S. Havlin, S. Kirkpatrick, Y. Shavitt, and E. Shir, 2006b, "MEDUSA - New model of Internet topology using $k$-shell decomposition," eprint cond-mat/0601240.

Carmi, S., S. Havlin, S. Kirkpatrick, Y. Shavitt, and E. Shir, 2007, "New model of Internet topology using $k$-shell decomposition," PNAS 104, 11150.

Caruso, F., V. Latora, A. Pluchino, A. Rapisarda, and B. Tadic, 2006, "Olami-Feder-Christensen model on different networks," Eur. Phys. J. B 50, 243.

Caruso, F., A. Pluchino, V. Latora, S. Vinciguerra, and A. Rapisarda, 2007, "Analysis of self-organized criticality in the Olami-Feder-Christensen model and in real earthquakes," Phys. Rev. E 75, 055101 (R).

Castellani, T., F. Krząkała, and F. Ricci-Tersenghi, 2005, "Spin glass models with ferromagnetically biased couplings on the Bethe lattice: analytic solutions and numerical sim- ulations," Eur. Phys. J. B 47, 99.

Castellano, C., V. Loreto, A. Barrat, F. Cecconi, and D. Parisi, 2005, "Comparison of voter and Glauber ordering dynamics on networks," Phys. Rev. E 71, 066107.

Castellano, C. and R. Pastor-Satorras, 2006a, "Non meanfield behavior of the contact process on scale-free networks," Phys. Rev. Lett. 96, 038701.

Castellano, C. and R. Pastor-Satorras, 2006b, "Zero temperature Glauber dynamics on complex networks," J. Stat. Mech. P05001.

Castellano, C. and R. Pastor-Satorras, 2007a, "Castellano and Pastor-Satorras reply," Phys. Rev. Lett. 98, 029802.

Castellano, C. and R. Pastor-Satorras, 2007b, "Routes to thermodynamic limit on scale-free networks," eprint arXiv:0710.2784.

Castellano, C., D. Vilone, and A. Vespignani, 2003, "Incomplete ordering of the voter model on small-world networks," Europhys. Lett. 63, 153.

Catanzaro, M., M. Boguñá, and R. Pastor-Satorras, 2005, "Diffusion-annihilation processes in complex networks," Phys. Rev. E 71, 056104.

Chalupa, J., P. L. Leath, and G. R. Reich, 1979, "Bootstrap percolation on a Bethe lattice," J. Phys. C 12, L31.

Chatterjee, A. and P. Sen, 2006, "Phase transitions in Ising model on a Euclidean network," Phys. Rev. E 74, 036109.

Chavez, M., D.-U. Hwang, A. Amann, H. G. E. Hentschel, and S. Boccaletti, 2005, "Synchronization is enhanced in weighted complex networks," Phys. Rev. Lett. 94, 218701.

Chavez, M., D.-U. Hwang, J. Martinerie, and S. Boccaletti, 2006, "Degree mixing and the enchancement of synchronization in complex weighted networks," Phys. Rev. E 74, 066107.

Chertkov, M. and V. Y. Chernyak, 2006a, "Loop series for discrete statistical models on graphs," J. Sta. Mech. P06009.

Chertkov, M. and V. Y. Chernyak, 2006b, "Loop calculus helps to improve belief propagation and linear programming decodings of low-density-parity-check codes," eprint cs.IT/0609154.

Chung, F. R. K., 1997, Spectral graph theory (American Mathematical Society, Providence, Rhode Island).

Chung, F. and L. Lu, 2002, "The average distances in random graphs with given expected degrees," PNAS 99, 15879.

Chung, F., L. Lu, and V. Vu, 2003, "Spectra of random graphs with given expected degrees," PNAS 100, 6313.

Clauset, A., M. E. J. Newman, and C. Moore, 2004, "Finding community structure in very large networks," Phys. Rev. E 70, 066111.

Cohen, R., D. ben-Avraham, and S. Havlin, 2002, "Percolation critical exponents in scale-free networks," Phys. Rev. E 66, 036113.

Cohen, R., K. Erez, D. ben-Avraham, and S. Havlin, 2000, "Resilience of the Internet to random breakdowns," Phys. Rev. Lett. 85, 4626.

Cohen, R., K. Erez, D. ben-Avraham, and S. Havlin, 2001, "Breakdown of the Internet under intentional attack," Phys. Rev. Lett. 86, 3682.

Cohen, R., S. Havlin, and D. ben-Avraham, 2003a, "Structural properties of scale free networks," in Handbook of Graphs and Networks, edited by S. Bornholdt and H. G. Schuster (Wiley-VCH GmbH \& Co., Weinheim), p. 85.

Cohen, R., S. Havlin, and D. ben-Avraham, 2003b, "Efficient immunization strategies for computer networks and populations," Phys. Rev. Lett. 91, 247901. 
Colizza, V., A. Barrat, M. Barthelemy, A.-J. Valleron, and A. Vespignani, 2007, "Modeling the worldwide spread of pandemic influenza: Baseline case and containment interventions," PLoS Med. 4, e13.

Colizza, V., A. Flammini, M. A. Serrano, and A. Vespignani, 2006, "Detecting rich-club ordering in complex networks," Nature Physics 2, 110.

Colizza, V., R. Pastor-Satorras, and A. Vespignani, 2007, "Reaction-diffusion processes and metapopulation models in heterogeneous networks," Nature Physics 3, 276.

Coolen, A. C. C., N. S. Skantzos, I. P. Castillo, C. J. P. Vicente, J. P. L. Hatchett, B. Wemmenhove, and T. Nikoletopoulos, 2005, Finitely connected vector spin systems with random matrix interactions, J. Phys. A 38, 8289.

Copelli, M. and P. R. A. Campos, 2007, "Excitable scale free networks," Eur. Phys. J. B 56, 273.

Coppersmith, D., D. Gamarnik, M. T. Hajiaghayi, and G. B. Sorkin, 2004, "Random MAX SAT, random MAX CUT, and their phase transitions," Random Structures and Algorithms 24, 502.

Costin, O. and R. D. Costin, 1991, "Limit probability distributions for an infinite-order phase transition model," J. Stat. Phys. 64, 193.

Costin, O., R. D. Costin, and C. P. Grünfeld, 1990, "Infiniteorder phase transition in a classical spin system," J. Stat. Phys. 59, 1531.

Coulomb, M. and S. Bauer, 2003, " Asymmetric evolving random networks," Eur. Phys. J. B 35, 377.

Danila, B., Y. Yu, S. Earl, J. A. Marsh, Z. Toroczkai, and K. E. Bassler, 2006, "Congestion-gradient driven transport on complex networks," Phys. Rev. E 74, 046114.

Denker, M., M. Timme, M. Diesmann, F. Wolf, and T. Geisel, 2004, "Breaking synchrony by heterogeneity in complex networks," Phys. Rev. Lett. 92, 074103.

Derényi, I., I. Farkas, G. Palla, and T. Vicsek, 2004, "Topological phase transitions of random networks," Physica A 334, 583 .

Derényi, I., G. Palla, and T. Vicsek, 2005, "Clique percolation in random networks," Phys. Rev. Lett. 94, 160202.

Derrida, B. 1981, "Random-energy model: An exactly solvable model of disordered systems," Phys. Rev. B 24, 2613.

Dezsõ, Z. and A.-L. Barabási, 2002, "Halting viruses in scalefree networks," Phys. Rev. E 65, 055103.

Dhar, D., P. Shukla, and J. P. Sethnal, 1997, "Zerotemperature gysteresis in the random field Ising model on a Bethe lattice," J. Phys. A 30, 5259.

Domb, C., 1960, "On the theory of cooperatve phenomena in crystals," Adv. Phys. 9, 245.

Dorogovtsev, S. N., 2003, "Renormalization group for evolving networks," Phys. Rev. E 67, 045102 (R).

Dorogovtsev, S. N., A. V. Goltsev, and J. F. F. Mendes, 2002a, "Pseudofractal scale-free web," Phys. Rev. E 65, 066122.

Dorogovtsev, S. N., A. V. Goltsev, and J. F. F. Mendes, 2002b, "Ising model on networks with an arbitrary distribution of connections," Phys. Rev. E 66, 016104.

Dorogovtsev, S. N., A. V. Goltsev, and J. F. F. Mendes, 2004, "Potts model on complex networks," Eur. Phys. J. B 38, 177.

Dorogovtsev, S. N., A. V. Goltsev, and J. F. F. Mendes, 2005, "Correlations in interacting systems with a network topology," Phys. Rev. E 72, 066130.

Dorogovtsev, S. N., A. V. Goltsev, and J. F. F. Mendes, 2006a, " $k$-core organization of complex networks," Phys.
Rev. Lett. 96, 040601.

Dorogovtsev, S. N., A. V. Goltsev, and J. F. F. Mendes, $2006 \mathrm{~b}$, " $k$-core architecture and $k$-core percolation on complex networks," Physica D 224, 7.

Dorogovtsev, S. N., A. V. Goltsev, J. F. F. Mendes, and S. N. Samukhin, 2003, "Spectra of complex networks," Phys. Rev. E 68, 046109.

Dorogovtsev, S. N., P. L. Krapivsky, and J. F. F. Mendes, 2007, "Transition from a small to a large world in growing networks," eprint arXiv:0709.3094.

Dorogovtsev, S. N. and J. F. F. Mendes, 2001, "Scaling properties of scale-free evolving networks: Continuous approach," Phys. Rev. E 63, 056125.

Dorogovtsev, S. N. and J. F. F. Mendes, 2002, "Evolution of networks," Adv. Phys. 51, 1079.

Dorogovtsev, S. N., and J. F. F. Mendes, 2003, Evolution of Networks: From Biological Nets to the Internet and $W W W$ (Oxford University Press, Oxford).

Dorogovtsev, S. N., J. F. F. Mendes, A. M. Povolotsky, and A. N. Samukhin, 2005, "Organization of complex networks without multiple connections," Phys. Rev. Lett. 95, 195701.

Dorogovtsev, S. N., J. F. F. Mendes, and A. N. Samukhin, 2000, "Exact solution of the Barabási-Albert model," Phys. Rev. Lett. 85, 4633.

Dorogovtsev, S. N., J. F. F. Mendes, and A. N. Samukhin, 2001a, "Giant strongly connected component of directed networks," Phys. Rev. E 64, 025101 (R).

Dorogovtsev, S. N., J. F. F. Mendes, and A. N. Samukhin, 2001b, "Anomalous percolation properties of growing networks," Phys. Rev. E 64, 066110.

Dorogovtsev, S. N., J. F. F. Mendes, and A. N. Samukhin, 2001c, "Size-dependent degree distribution of a scale-free growing network," Phys. Rev. E 63, 062101.

Dorogovtsev, S. N., J. F. F. Mendes, and A. N. Samukhin, 2003a, "Metric structure of random networks," Nucl. Phys. B 653, 307.

Dorogovtsev, S. N., J. F. F. Mendes, and A. N. Samukhin, 2003b, "Principles of statistical mechanics of random networks," Nucl. Phys. B 666, 396.

Doye, J. P. K. and C. P. Massen, 2005, "Self-similar disk packings as model spatial scale-free networks," Phys. Rev. E 71, 016128.

Durrett, R., 2006, Random Graph Dynamics (Cambridge University Press, Cambridge).

Echenique, P., J. Gómez-Gardeñes, and Y. Moreno, 2005, "Dynamics of jamming transitions in complex networks," Europhys. Lett. 71, 325.

Eggarter, T. P., 1974, "Cayley trees, the Ising problem, and the thermodynamic limit," Phys. Rev. B 9, 2989.

Ehrhardt, G. C. M. A. and M. Marsili, 2005, "Potts model on random trees," J. Stat. Mech.: Theor. Exp. P02006.

Ehrhardt, G. C. M. A., M. Marsili, and F. Vega-Redondo, 2006, "Phenomenological models of socioeconomic network dynamics," Phys. Rev. E 74, 036106.

Erdős, P. and A. Rényi, 1959, "On random graphs," Publicationes Mathematicae Debrencen 6, 290.

Evans, M. R. and T. Hanney, 2005, "Nonequilibrium statistical mechanics of the zero-range process and related models," J. Phys. A 38, R195.

Falk, H. 1975, "Ising spin system on a Cayley tree: Correlation decomposition and phase transition," Phys. Rev. B 12, 5184 .

Farkas, I., I. Derenyi, G. Palla, and T. Vicsek, 2004, "Equi- 
librium statistical mechanics of network structures," Lect. Notes Phys. 650, 163.

Fernholz, D. and V. Ramachandran, 2004, "Cores and connectivity in sparse random graphs," UTCS Technical Report TR04-13.

Fortuin, C. M. and P. W. Kasteleyn, 1972, "On the randomcluster model: I. Introduction and relation to other models," Physica 57, 536.

Freeman, W. T., E. C. Pasztor, and O. T. Carmichael, 2000, "Learning low-level vision," Int. J. Comput. Vis. 40, 25.

Frey, B. J., 1998, Graphical models for machine learning and digital communication (MIT Press, Cambridge).

Frieze, A. M. 1990, "On the independence number of random graphs," Discr. Math. 81, 171.

Fronczak, A. and P. Fronczak, 2007, "Biased random walks on complex networks: the role of local navigation rules," eprint arXiv:0708.4404 [math]

Gade, P. M. and C.-K. Hu, 2000, "Synchronous chaos in coupled map lattices with small-world interactions," Phys. Rev. E 62, 6409.

Galla, T., M. Leone, M. Marsili, M. Sellitto, M. Weigt, and R. Zecchina, 2006, "Statistical mechanics of combinatorial auctions," Phys. Rev. Lett. 97, 128701.

Gallos, L. K. and P. Argyrakis, 2004, "Absence of kinetic effects in reaction-diffusion processes in scale-free networks," Phys. Rev. Lett. 92, 138301.

Gallos, L. K., R. Cohen, P. Argyrakis, A. Bunde, and S. Havlin, 2005, "Stability and topology of scale-free networks under attack and defense strategies," Phys. Rev. Lett. 94, 188701.

Gallos, L. K., F. Liljeros, P. Argyrakis, A. Bunde, and S. Havlin, 2007a, "Improving immunization strategies," eprint arXiv:0704.1589 [cond-mat].

Gallos, L. K., C. Song, S. Havlin, and H. A. Makse, 2007b, "Scaling theory of transport in complex networks," PNAS 104, 7746 .

Garey, M. A. and D. S. Johnson, 1979, Computers and intractability (Freeman, New York).

Gil, S. and D. H. Zanette, 2006, "Coevolution of agents and networks: Opinion spreading and community disconnection," Phys. Lett. A 356, 89.

Gilbert, E. N., 1959, "Random graphs," Ann. Math. Stat. 30, 1141.

Gitterman, M. 2000, "Small-word phenomena in physics: the Ising model," J. Phys. A 33, 8373.

Giuraniuc, C. V., J. P. L. Hatchett, J. O. Indekeu, M. Leone, I. Pérez Castillo, B. Van Schaeybroeck, and C. Vanderzande, 2005, "Trading interactions for topology in scalefree networks," Phys. Rev. Lett. 95, 098701.

Giuraniuc, C. V., J. P. L. Hatchett, J. O. Indekeu, M. Leone, I. Perez Castillo, B. Van Schaeybroeck, and C. Vanderzande, 2006, "Criticality on networks with topologydependent interactions," Phys. Rev. E 74, 036108.

Goh, K.-I., B. Kahng, and D. Kim, 2001, "Universal behavior of load distribution in scale-free networks," Phys. Rev. Lett. 87, 278701.

Goh, K.-I., D.-S. Lee, B. Kahng, and D. Kim, 2003, "Sandpile on scale-free networks," Phys. Rev. Lett. 91, 148701.

Goh, K.-I., D.-S. Lee, B. Kahng, and D. Kim, 2005, "Cascading toppling dynamics on scale-free networks," Physica A 346, 011665.

Goh, K.-I., G. Salvi, B. Kahng, and D. Kim, 2006, "Skeleton and fractal scaling in complex networks," Phys. Rev. Lett. 96, 018701.
Goldschmidt, Y. Y. and C. De Dominicis, "Replica symmetry breaking in the spin-glass model on lattices with finite connectivity: Application to graph partitioning," 1990, Phys. Rev. B 41, 2184.

Goltsev, A. V., S. N. Dorogovtsev, and J. F. F. Mendes, 2003, "Critical phenomena in networks," Phys. Rev. E 67, 026123.

Goltsev, A. V., S. N. Dorogovtsev, and J. F. F. Mendes, 2006, " $k$-core (bootstrap) percolation on complex networks: Critical phenomena and nonlocal effects," Phys. Rev. E 73, 056101.

Gómez-Gardeñes, J., Y. Moreno, and A. Arenas, 2007a, "Paths to synchronization on complex networks," Phys. Rev. Lett. 98, 034101.

Gómez-Gardeñes, J., Y. Moreno, and A. Arenas, 2007b, "Synchronizability determined by coupling strengths and topology on complex networks," Phys. Rev. E 75, 066106.

Gourley, S. and N. F. Johnson, 2006, "Effects of decisionmaking on the transport costs across complex networks," Physica A 363, 82.

Grassberger P., 1983, "Critical behavior of the general epidemic process and dynamical percolation," Math. Biosci. 63, 157.

Grassberger, P. and A. de la Torre, 1979, "Reggeon field theory (Schlögl's first model) on a lattice: Monte Carlo calculations of critical behaviour," Ann. Phys. (NY) 122, 373.

Grinstein, G. and R. Linsker, 2005, "Synchronous neural activity in scale-free network models versus random network models," Proc. Natl. Acad. Sci. 102, 9948.

Gross, T. and B. Blasius, 2007, "Adaptive coevolutionary networks - a review," eprint arXive:0709.1858.

Guardiola, X., A. Díaz-Guilera, M. Llas, and C. J. Pérez, 2000, "Synchronization, diversity, and topology of networks of integrate and fire oscillators," Phys. Rev. E 62, 5565.

Guimerà, R., A. Díaz-Guilera, F. Vega-Redondo, A. Cabrales, and A. Arenas, 2002, "Optimal network topologies for local search with congestion," Phys. Rev. Lett. 89, 258701.

Guimerà, R., M. Sales-Pardo, and L. A. N. Amaral, 2004, "Modularity from fluctuations in random graphs and complex networks," Phys. Rev. E 70, 025101.

Ha, M., H. Hong, and H. Park, 2007, "Comment on 'nonmean-field behavior of the contact process on scale-free networks'," Phys. Rev. Lett. 98, 029801.

Häggström O., 2002, "Zero-temperature dynamics for the ferromagnetic Ising model on random graphs," Physica A 310, 275.

Harris T. E., 1974, "Contact interactions on a lattice," Ann. Probab. 2, 969.

Harris, A. B. 1975, "Nature of the Griffiths singularity in dilute magnets," Phys. Rev. B 12, 203.

Harris A. B., 1982, "Exact solution of a model of localization," Phys. Rev. Lett. 49, 296.

Hartmann A. K. and M. Weigt, 2005, Phase Transitions in Combinatorial Optimization Problems: Basics, Algorithms and Statistical Mechanics (Wiley-VCH).

Hase, M. O., J. R. L. Almeida, and S. R. Salinas, 2005, "Replica-symmetric solutions of a dilute Ising ferromagnet in a random field," Eur. Phys. J B 47, 245.

Hase, M. O., J. R. L. de Almeida, and S. R. Salinas, 2006, "Spin glass behaviour on random lattices," eprint condmat/0604144.

Hastings, M. B., 2003, "Mean-field and anomalous behavior on a small-world network," Phys. Rev. Lett. 91, 098701.

Hastings M. B., 2006, "Systematic series expansions for pro- 
cesses on networks," Phys. Rev. Lett. 96, 148701.

von Heimburg, J. and H. Thomas, 1974, "Phase transition of the Cayley tree with Ising interaction," J. Phys. C 7, 3433.

Helbing, D., J. Siegmeier, and S. Lämmer, 2007, "Selforganized network flows," Networks and Heterogeneous Media 2, 193.

Herrero, C. P., 2002, "Ising model in small-world networks," Phys. Rev. E 65, 066110.

Herrero, C. P. 2004, "Ising model in scale-free networks: A Monte Carlo simulation," Phys. Rev. E 69, 067109.

Hinczewski, M. and A. N. Berker, 2006, "Inverted BerezinskiiKosterlitz-Thouless singularity and high-temperature algebraic order in an Ising model on a scale-free hierarchicallattice small-world network," Phys. Rev. E 73, 066126.

Hinrichsen, H., 2000, "Non-equilibrium critical phenomena and phase transitions into absorbing states," Adv. Phys. 49, 815 .

Holme, P., 2003, "Congestion and centrality in traffic flow on complex networks," Adv. Complex Systems 6, 163.

Holme P., 2007, "Scale-free networks with a large- to hypersmall-world transition," Physica A 377, 315.

Holme, P., F. Liljeros, C. R. Edling, and B. J. Kim,, 2003, "Network bipartivity," Phys. Rev. E 68, 056107.

Holme, P. and M. E. J. Newman, 2006, "Nonequilibrium phase transition in the coevolution of networks and opinions," Phys. Rev. E 74, 056108.

Hong, H., H. Chaté, H. Park, and L.-H. Tang, 2007b, "Entrainment transition in populations of random frequency oscillators," Phys. Rev. Lett. 99, 184101.

Hong, H., M. Y. Choi, and B. J. Kim, 2002a, "Synchronization on small-world networks," Phys. Rev. E 65, 026139.

Hong, H., M. Ha, and H. Park, 2007a, "Finite-size scaling in complex networks," Phys. Rev. Lett. 98, 258701.

Hong, H., B. J. Kim, and M. Y. Choi, 2002b, "Comment on Ising model on a small world network," Phys. Rev. E 66, 018101.

Hong, H., B. J. Kim, M. Y. Choi, and H. Park, 2004a, "Factors that predict better synchronizability on complex networks," Phys. Rev. E 69, 067105.

Hong, H., H. Park, and Choi M. Y., 2004b, "Collective phase synchronization in locally coupled limit-cycle oscillators," Phys. Rev. E 70, 045204 (R).

Hong, H., H. Park, and Choi M. Y., 2005, "Collective synchronization in spatially extended systems of coupled oscillators with random frequencies," Phys. Rev. E 72, 036217.

Hong, H., H. Park, and L.-H. Tang, 2007c, "Finite-size scaling of synchronized oscillation on complex networks," eprint arXiv:0710.1137.

Hovorka, O. and G. Frieman, 2007, "Non-converging hysteretic cycles in random spin networks," eprint condmat/0703525.

Huang, L., K. Park, Y.-C. Lai, L. Yang, and K. Yang, 2006, "Abnormal synchronization in complex clustered networks," Phys. Rev. Lett. 97, 164101.

Ichinomiya, T. 2004, "Frequency synchronization in a random oscillator network," Phys. Rev. E 70, 026116.

Ichinomiya, T. 2005, "Path-integral approach to the dynamics in sparse random network," Phys. Rev. E 72, 016109.

Iglói, F. and L. Turban, 2002, "First- and second-order phase transitions in scale-free networks," Phys. Rev. E 66, 036140 .

Ihler, A. T., J. W. Fischer, and A. S. Willsky, 2005, "Loopy belief propagation: convergence and effects of message errors," J. Mach. Learning Res. 6, 905.
Imry, Y. and S.-K. Ma, 1975, "Random-field instability of the ordered state of continuous symmetry," Phys. Rev. Lett. 35, 1399.

Janson, S., 2007, "The largest component in a subcritical random graph with a power law degree distribution," eprint arXiv:0708.4404 [math].

Jeong, D., H., Hong, B. J. Kim, and M. Y. Choi, 2003, "Phase transition in the Ising model on a small-world network with distance-dependent interactions," Phys. Rev. E 68, 027101.

Johnston, D. A. and P. Plecháč, 1998, "Equivalence of ferromagnetic spin models on trees and random graphs," J. Phys. A 31, 475.

Jost, J. and M. P. Joy, 2001, "Spectral properties and synchronization in coupled map lattices," Phys. Rev. E 65, 016201.

Jung, S., S. Kim, and B. Kahng, 2002, "Geometric fractal growth model for scale-free networks," Phys. Rev. E 65, 056101.

Kalapala, V. and C. Moore, 2002, "MAX-CUT on sparse random graphs," http://www.cs.unm.edu/ treport/tr/0208/maxcut.ps.gz.

Kalisky, T. and R. Cohen, 2006, "Width of percolation transition in complex networks," Phys. Rev. E 73, 035101.

Kanter, I. and H. Sompolinsky, 2000, "Mean-field theory of spin-glasses with finite coordination number," Phys. Rev. Lett. 58, 164.

Kappen, H., 2002, in Modelling Biomedical Signals, edited by G. Nardulli and S. Stramaglia (World Scientific, Singapore), p. 3-27.

Karp, R. M. and M., Sipser, 1981, "Maximum matchings is sparse random graphs," in Proceedings of the 22nd IEEE Symposium on Foundations of Computing, p. 364.

Karsai, M., J.-C. A. d'Auriac, and F. Iglói, 2007, "Rounding of first-order phase transitions and optimal cooperation in scale-free networks," eprint arXiv:0704.1538 [cond-mat].

Karsai, M., R. Juhász, and F. Iglói, 2006, "Nonequilibrium phase transitions and finite size scaling in weighted scalefree networks," Phys. Rev. E 73, 036116.

Kasteleyn, P. W. and C. M. Fortuin, 1969, "Phase transition in lattice systems with random local properties," J. Phys. Soc. Jpn. Suppl. 26, 11.

Kenah, E. and J. Robins, 2006, "Second look at the spread of epidemics on networks," eprint q-bio/0610057.

Khajeh, E., S. N. Dorogovtsev, and J. F. F. Mendes, 2007, "BKT-like transition in the Potts model on an inhomogeneous annealed network," Phys. Rev. E 75, 041112.

Kikuchi, R., 1951, "A theory of cooperative phenomena," Phys. Rev. 81, 988.

Kim, B. J., 2004, "Performance of networks of artificial neurons: the role of clustering," Phys. Rev. E 69, 045101.

Kim, B. J., H. Hong, P. Holme, G. S Jeon, P. Minnhagen, and M. Y. Choi, 2001, "XY model in small-world networks," Phys. Rev. E 64, 056135.

Kim, J., P. L. Krapivsky, B. Kahng, and S. Redner, 2002, "Infinite-order percolation and giant fluctuations in a protein interaction network," Phys. Rev. E 66, 055101.

Kim, D.-H. and A. E. Motter, 2007, "Ensemble averageability in network spectra," Phys. Rev. Lett. 98, 248701.

Kim, D.-H., G. J. Rodgers, B. Kahng, and D. Kim, 2005, "Spin glass transition on scale-free networks," Phys. Rev. E 71, 056115.

Kim, B. J., A. Trusina, P. Minnhagen, and K. Sneppen, 2005, "Self organized scale-free networks from merging and regeneration," Eur. Phys. J. B 43, 369. 
Kinouchi, O. and M. Copelli, 2006, "Optimal dynamical range of excitable networks at criticality," Nat. Phys. 2, 348.

Kirkpatrick, S., 2005, "Jellyfish and other interesting creatures of the Internet," http://www.cs.huji.ac.il/ ${ }^{\sim}$ kirk/Jellyfish_Dimes.ppt.

Kleinberg, J., 1999, "The small-world phenomenon: An algorithmic perspective.," Cornell Computer Science Technical Report 99-1776 (October 1999).

Kleinberg J., 2000, "Navigation in a small world," Nature 406, 845 .

Kleinberg J., 2006, "Complex networks and decentralized search algorithms," http://www.cs.cornell.edu/home/kleinber/icm06-swn.pdf.

Klemm, K., V. M. Eguíluz, R. Toral, and M. San Miguel, 2003, "Nonequilibrium transitions in complex networks: A model of social interaction," Phys. Rev. E 67, 026120.

Kori, H. and A. S. Mikhailov, 2004, "Entrainment of randomly coupled oscillator networks by a pacemaker," Phys. Rev. Lett. 93, 254101.

Kori, H. and A. S. Mikhailov, 2006, "Strong effect of network architecture of coupled oscillator systems," eprint condmat/0608702

Kosterlitz, J. M. and D. J. Thouless, 1973, "Ordering, metastability and phase transitions in two-dimensional systems," J. Phys. C 6, 1181.

Kozma, B. and A. Barrat, 2007, "Consensus formation on adaptive networks," eprint arXiv:0707.4416 [physics].

Kozma, B., M. B. Hastings, and G. Korniss, 2004, "Roughness scaling for Edwards-Wilkinson relaxation in small-world networks," Phys. Rev. Lett. 92, 108701.

Krapivsky P. L., 1992, "Kinetics of monomer-monomer surface catalytic reactions," Phys. Rev. A 45, 1067.

Krapivsky, P. L. and B. Derrida, 2004, "Universal properties of growing networks," Physica A 340, 714.

Krapivsky, P. L. and S. Redner, 2002, "Finiteness and fluctuations in growing networks," J. Phys. A 35, 9517.

Krapivsky, P. L., S. Redner, and F. Leyvraz, 2000, "Connectivity of growing random networks," Phys. Rev. Lett. 85, 4629.

Krivelevich, M. and B. Sudakov, 2003, "The largest eigenvalue of sparse random graphs," Combinatorics, Probab. Comput. 12, 61.

Krząkała, F., A. Montanari, F. Ricci-Tersenghi, G. Semerjian, and L. Zdeborova, 2007, "Gibbs states and the set of solutions of random constraint satisfaction problems," PNAS 104, 10318.

Krzạkała, F., A. Pagnani, and M. Weigt, 2004, "Threshold values, stability analysis, and high-q asymptotics for the coloring problem on random graphs," Phys. Rev. E 70, 046705.

Kulkarni, R. V., E., Almaas, and D. Stroud, 1999, "Evolutionary dynamics in the Bak-Sneppen model on small-world networks," eprint cond-mat/9905066.

Kumpula, J. M., J. Saramaki, K. Kaski, and J. Kertesz, 2007, "Limited resolution in complex network community detection with Potts model approach," Eur. Phys. J. B 56, 41.

Kuramoto, Y., 1984, Chemical Oscillations, Waves and Turbulence (Springer, New York).

Kwon, O. and H.-T. Moon, 2002, "Coherence resonance in small-world networks of excitable cells," Phys. Lett. A 298, 319.

Kwon, C. and D. J. Thouless, 1988, "Ising spin glass at zero temperature on the Bethe lattice," Phys. Rev. B 37, 7649.

Lacour-Gayet, P. and G. Toulouse, 1974, "Ideal Bose-Einstein condensation and disorder effects," J. Phys. (Paris) 35, 425.

Lago-Fernández, L. F., R. Huerta, F. Corbacho, and J. A. Sigüenza, 2000, "Fast response and temporal coherent oscillations in small-world networks," Phys. Rev. Lett. 84, 2758.

Lahtinen, J., J. Kertész, and K. Kaski, 2005, "Sandpiles on Watts-Strogatz type small-worlds," Physica A 349, 535.

Lambiotte, R. and M. Ausloos, 2005, "Uncovering collective listening habits and music genres in bipartite networks," Phys. Rev. E 72, 066107.

Lancaster, D., 2002, "Cluster growth in two growing network models," J. Phys. A 35, 1179.

Lee, D.-S. 2005, "Synchronization transition in scale-free networks: Clusters of synchrony," Phys. Rev. E 72, 026208.

Lee, D.-S., K.-I. Goh, B. Kahng, and D. Kim, 2004a, "Sandpile avalanche dynamics on scale-free networks,", Physica A 338, 84.

Lee, D.-S., K.-I. Goh, B. Kahng, and D. Kim, 2004b, "Branching process approach to avalanche dynamics on complex networks," J. Korean Phys. Soc. 44, 633.

Lee, D.-S., K.-I. Goh, B. Kahng, and D. Kim, 2004c, "Evolution of scale-free random graphs: Potts model formulation," Nucl. Phys. B 696, 351.

Lee, E. J., K.-I. Goh, B. Kahng, and D. Kim, 2005a, "Robustness of the avalanche dynamics in data packet transport on scale-free networks," Phys. Rev. E 71, 056108.

Lee, K. E., B. H. Hong, and J. W. Lee, 2005b, "Universality class of Bak-Sneppen model on scale-free network," eprint cond-mat/0510067.

Lee, J.-S., K.-I. Goh, B. Kahng, and D. Kim, 2006a, "Intrinsic degree-correlations in static model of scale-free networks," Eur. Phys. J. B 49, 231.

Lee, S. H., H. Jeong, and J. D. Noh, 2006b, "Random field Ising model on networks with inhomogeneous connections," Phys. Rev. E 74, 031118.

Leland, W. E., M. S. Taqqu, W. Willinger, and D. W. Wilson, 1994, "On the self-similar nature of Ethernet traffic (extended version)," IEEE/ACM Trans. Network. 2, 1.

Leone, M., A. Vázquez, A. Vespignani, and R. Zecchina, 2002, "Ferromagnetic ordering in graphs with arbitrary degree distribution," Eur. Phys. J. B 28, 191.

Li, G., L. A. Braunstein, S. V. Buldyrev, S. Havlin, and H. E. Stanley, 2007, "Transport and percolation theory in weighted networks," Phys. Rev. E 75, 045103.

Liers, F., M. Palassini, A. K. Hartmann, and M. Jünger, 2003, "Ground state of the Bethe lattice spin glass and running time of an exact optimization algorithm," Phys. Rev. B 68, 094406.

Lima, F. W. S. and D. Stauffer, 2006, "Ising model simulations in directed lattices and networks," Physica A 359, 423.

Lind, P. G., J. A. C. Gallas, and H. J. Herrmann, 2004, "Coherence in scale-free networks of chaotic maps," Phys. Rev. E 70, 056207.

Liu, Z., W. Ma, H. Zhang, Y. Sun, and P. M. Hui, 2006, "An efficient approach of controlling traffic congestion in scale-free networks," Physica A 370, 843.

Lopes, J. V., Y. G. Pogorelov, J. M. B. L. dos Santos, and R. Toral, 2004, Phys. Rev. E 70, 026112.

López, E., R. Parshani, R. Cohen, S. Carmi, and S. Havlin, 2007, "Limited path percolation in complex networks," eprint cond-mat/0702691.

Lübeck, S. and H.-K. Janssen, 2005, "Finite-size scaling of directed percolation above the upper critical dimension," 
Phys. Rev. E 72, 016119.

Luczak, T. 1991, "The chromatic number of random graphs," Combinatorica 11, 45.

Luijten, E. and H. W. J. Blöte, 1997, "Classical critical behavior of spin models with long-range interactions," Phys. Rev. B 56, 8945.

Lyons, R., 1989, "The Ising model and percolation on trees and tree-like graphs," Commun. Math. Phys. 125, 337.

Lyons R., 1990, "Random walks and percolation on trees," Ann. Probab. 18, 931.

Lyons, R., R. Pemantle, and Y. Peres, 1996, "Biased random walks on Galton-Watson trees," Prob. Theor. Relat. Fields 106, 249 .

Malarz K., W. Antosiewicz, J. Karpinska, K. Kulakowski, and B. Tadić, 2007, "Avalanches in complex spin networks," Physica A 373, 785.

Martel, C. and V., Nguyen, 2004, "Analyzing Kleinberg's (and other) small-world models," in Proceedings of the twentythird annual ACM symposium on Principles of distributed computing, St. John's, Newfoundland, Canada, p. 179.

Maslov, S. and K. Sneppen, 2002, "Specificity and stability in topology of protein networks," Science 296, 910.

Masuda, N., K.-I. Goh, and B. Kahng, 2005, "Extremal dynamics on complex networks: Analytic solutions," Phys. Rev. E 72, 066106.

Matsuda, H. 1974, "Infinite susceptibility without spontaneous magnetization: Exact properties of the Ising model on the Cayley tree," Prog. Theor. Phys. 51, 1053.

May R. M. and A. L. Lloyd, 2001, "Infection dynamics on scale-free networks," Phys. Rev. E 64, 066112.

McEliece, R. J., D. J. C. MacKay, and J. F. Cheng, 1998, "Turbo decoding as an instance of Pearl's beliefpropagation algorithm ," IEEE J. Select. Areas Commun. 16, 140.

McGraw, P. and M. Menzinger, 2006, "Analysis of nonlinear synchronization dynamics of oscillator networks by Laplacian spectral methods," eprint cond-mat/0610522.

Medvedyeva, K., P. Holme, P. Minnhagen, and B. J. Kim, 2003, "Dynamic critical behavior of the XY model in smallworld networks," Phys. Rev. E 67, 036118.

Melin, R., J. C. Angles d'Auriac, P. Chandra, and B. Doucot, 1996, "Glassy behavior in the ferromagnetic Ising model on a Cayley tree," J. Phys. A 29, 5773.

Mertens, S., M. Mézard, and R. Zecchina, 2003, "Threshold values of Random K-SAT from the cavity method," Rand. Struct.and Alg. 28, 340 .

Mézard, M., T. Mora, and R. Zecchina, 2005, "Clustering of solutions in the random satisfiability problem," Phys. Rev. Lett. 94, 197205.

Mézard, M. and G. Parisi, 2001, "The Bethe lattice spin glass revisited," Eur. Phys. J. B 20, 217.

Mézard, M. and G. Parisi, 2003, "The cavity method at zero temperature," J. Stat. Phys. 111, 1.

Mézard, M., G. Parisi, and M. A. Virasoro, 1987, Spin glass theory and beoyond (World Scientific, Singapur).

Mézard, M., G. Parisi, and R. Zecchina, 2002, "Analytic and algorithmic solution of random satisfiability problems," Science 297, 812 .

Mézard, M. and M. Tarzia, 2007, "Statistical mechanics of the hyper vertex cover problem," eprint arXiv:0707.0189 [cond-mat].

Mézard, M. and R. Zecchina, 2002, "Random K-satisfiability problem: From an analytic solution to an efficient algorithm," Phys. Rev. E 66, 056126.

Miller, J. C., 2007, "Epidemic size and probability in popu- lations with heterogeneous infectivity and susceptibility," Phys. Rev. E 76, 010101 (R).

Minnhagen, P., M. Rosvall, K. Sneppen, and A. Trusina, 2004, "Self-organization of structures and networks from merging and small-scale fluctuations," Physica A 340, 725.

Molloy, M. and B. A. Reed, 1995, "A critical point for random graphs with a given degree sequence," Random Struct. Algor. 6, 161.

Molloy, M. and B. A. Reed, 1998, "The size of the giant component of a random graph with a given degree sequence," Combin. Prob. Comp. 7, 295.

Montanari, A., 2005, "Two lectures on iterative coding and statistical mechanics," eprint cond-mat/0512296.

Montanari, A. and T. Rizzo, 2005, "How to compute loop corrections to Bethe approximation," J. Stat. Mech. P10011.

Mooij, J. M. and H. J. Kappen, 2005, "On the properties of the Bethe approximation and loopy belief propagation on binary networks," J. Stat. Mech. P11012.

Moore, C. and M. E. J. Newman, 2000a, "Epidemics and percolation in small-world networks," Phys. Rev. E 61, 5678.

Moore, C. and M. E. J. Newman, 2000b, "Exact solution of site and bond percolation on small-world networks," Phys. Rev. E 62, 7059.

Moreno, Y. and A. F. Pacheco, 2004, "Synchronization of Kuramoto oscillators in scale-free networks," Europhys. Lett. 68, 603.

Moreno, Y., R. Pastor-Satorras, A. Vázquez, and A. Vespignani, 2003, "Critical load and congestion instabilities in scale-free networks," Europhys. Lett. 62, 292.

Moreno, Y., R. Pastor-Satorras, and A. Vespignani, 2002, "Epidemic outbreaks in complex heterogeneous networks," Eur. Phys. J. B 26, 521.

Moreno, Y. and A. Vazquez, 2002, "The Bak-Sneppen model on scale-free networks," Europhys. Lett. 57, 765.

Moreno, Y. and A. Vázquez, 2003, "Disease spreading in structured scale-free networks," Eur. Phys. J. B 31, 265.

Moreno, Y., M. Vazquez-Prada, and A. F. Pacheco, 2004, "Fitness for Synchronization of Network Motifs," Physica A 343, 279.

Motter, A. E., 2004, "Cascade control and defense in complex networks," Phys. Rev. Lett. 93, 098701.

Motter A. E., 2007, "Bounding network spectra for network design," New J. Phys. 9, 182.

Motter, A. E. and Y.-C. Lai, 2002, "Cascade-based attacks on complex networks," Phys. Rev. E 66, 065102.

Motter, A. E., C. Zhou, and J. Kurths, 2005a, "Network synchronization, diffusion, and the paradox of heterogeneity," Phys. Rev. E 71, 016116.

Motter, A. E., C. Zhou, and J. Kurths, 2005b, "Enhancing complex-network synchronization," Europhys. Lett. 69, 334 .

Motter, A. E., C. Zhou, and J. Kurths, 2005c, "Weighted networks are more synchronizable: How and why," AIP Conf. Proceedings 776, 201.

Mottishaw, P., 1987, "Replica symmetry breaking and the spin-glass on a Bethe lattice," Europhys. Lett. 4, 333.

Moukarzel, C. F. and M. A. de Menezes, 2002, "Shortest paths on systems with power-law distributed long-range connections," Phys. Rev. E 65, 056709.

Mukamel, D. 1974, "Two-spin correlation function of spin 1/2 Ising model on a Bethe lattice," Phys. Lett. A 50, 339.

Mulet, R., A. Pagnani, M. Weigt, and R. Zecchina, 2002, "Coloring random graphs," Phys. Rev. Lett. 89, 268701.

Muller-Hartmann, E. and J. Zittartz, 1974, "New type of 
phase transition," Phys. Rev. Lett. 33, 893.

Nâsell I., 2002, "Stochastic models of some endemic infections," Math. Biosci. 179, 1.

Newman, M. E. J., 2000, "Models of the small world," J. Stat. Phys. 101, 819.

Newman, M. E. J., 2002a, "The spread of epidemic disease on networks," Phys. Rev. E 66, 016128.

Newman, M. E. J., 2002b, "Assortative mixing in networks," Phys. Rev. Lett. 89, 208701.

Newman, M. E. J., 2003a, "The structure and function of complex networks," SIAM Review 45, 167.

Newman M. E. J., 2003b, "Properties of highly clustered networks," Phys. Rev. E 68, 026121.

Newman, M. E. J., 2003c, "Random graphs as models of networks," in Handbook of Graphs and Networks: From the Genome to the Internet, edited by $\mathrm{S}$. Bornholdt and $\mathrm{H}$. G. Schuster, (Wiley-VCH, Berlin), p. 35.

Newman, M. E. J. 2003d, "Mixing patterns in networks," Phys. Rev. E 67, 026126.

Newman M. E. J., 2007, "Component sizes in networks with arbitrary degree distributions," eprint arXiv:0707.0080 [cond-mat].

Newman, M. E. J. and M. Girvan, 2004, "Finding and evaluating community structure in networks," Phys. Rev. E 69, 026113.

Newman, M. E. J., I. Jensen, and R. M. Ziff, 2002, "Percolation and epidemics in a two-dimensional small world," Phys. Rev. E 65, 021904.

Newman, M. E. J., S. H. Strogatz, and D. J. Watts, 2001, "Random graphs with arbitrary degree distributions and their applications," Phys. Rev. E 64, 026118.

Newman, M. E. J. and D. J. Watts, 1999a, "Scaling and percolation in the small-world network model," Phys. Rev. E 60, 7332 .

Newman, M. E. J. and D. J. Watts, 1999b, "Renormalization group analysis of the small-world network model," Phys. Lett. A 263, 341.

Nikoletopoulos, T., A. C. C. Coolen, I. Pérez Castillo, N. S. Skantzos, J. P. L. Hatchett, and B. Wemmenhove, 2004, "Replicated transfer matrix analysis of Ising spin models on 'small world' lattices," J. Phys. A 37, 6455.

Nishikawa, T. and A. E. Motter, 2006a, "Synchronization is optimal in non-diagonalizable networks," Phys. Rev. E 73, 065106.

Nishikawa, T. and A. E. Motter, 2006b, "Maximum performance at minimum cost in network synchronization," Physica D 224, 77.

Nishikawa, T., A. E. Motter, Y.-C. Lai, and F. C. Hoppensteadt, 2003, "Heterogeneity in oscillator networks: Are smaller worlds easier to synchronize?," Phys. Rev. Lett. 91, 014101.

Noh, J. D., 2005, "Stationary and dynamical properties of a zero range process on scale-free networks," Phys. Rev. E 72, 056123.

Noh, J. D., 2007, "Percolation transition in networks with degree-degree correlation," eprint arXiv:0705.0087 [condmat].

Noh, J. D., G. M. Shim, and H. Lee, 2005, "Complete condensation in a zero range process on scale-free networks," Phys. Rev. Lett. 94, 198701.

Oh, E., D.-S. Lee, B. Kahng, and D. Kim, 2007, "Synchronization transition of heterogeneously coupled oscillators on scale-free networks," Phys. Rev. E 75, 011104.

Oh, E., K. Rho, H. Hong, and B. Kahng, 2005, "Modular synchronization in complex networks," Phys. Rev. E $\mathbf{7 2}$ 047101.

Ohira, T. and R. Sawatari, 1998, "Phase transition in a computer network traffic model," Phys. Rev. E 58, 193.

Ohkubo, J., M. Yasuda, and K. Tanaka, 2005, "Statisticalmechanical iterative algorithms on complex networks," Phys. Rev. E 72, 046135.

Ostilli, M., 2006a, "Ising spin glass models versus Ising models: an effective mapping at high temperature I. General result," J. Stat. Mech. P10004.

Ostilli M., 2006b, "Ising spin glass models versus Ising models: an effective mapping at high temperature II. Applications to graphs and networks," J. Stat. Mech. P10005.

Ozana M., 2001, "Incipient spanning cluster on small-world networks," Europhys. Lett. 55, 762.

Pacheco, J. M., A. Traulsen, and M. A. Nowak, 2006, "Coevolution of strategy and structure in complex networks with dynamical linking," Phys. Rev. Lett. 97, 258103.

Pagnani, A., G. Parisi, and M. Ratieville, 2003, "Metastable configurations on the Bethe lattice," Phys. Rev. E 67, 026116 .

Palla, G., I. Derényi, I. Farkas, and T. Vicsek, 2004, "Statistical mechanics of topological phase transitions in networks," Phys. Rev. E 69, 046117.

Palla, G., I. Derényi, and T. Vicsek, 2007, "The critical point of $k$-clique percolation in the Erdős-Rényi graph," J. Stat. Phys. 128, 219.

Parisi, G. and T. Rizzo, 2006, "On $k$-core percolation in four dimensions," eprint cond-mat/0609777.

Parisi, G. and F. Slanina, 2006, "Loop expansion around the Bethe-Peierls approximation for lattice models," J. Stat. Mech. 0602, L003.

Park, J. and M. E. J. Newman, 2004a, "Solution of the 2-star model of a network," Phys. Rev. E 70, 066146.

Park, J. and M. E. J. Newman, 2004b, "The statistical mechanics of networks," Phys. Rev. E 70, 066117.

Pastor-Satorras, R. and A. Vespignani, 2001, "Epidemic spreading in scale-free networks," Phys. Rev. Lett. 86, 3200 .

Pastor-Satorras, R. and A. Vespignani, 2002a, "Epidemic dynamics in finite size scale-free networks," Phys. Rev. E 65, 035108 .

Pastor-Satorras, R. and A. Vespignani, 2002b, "Immunization of complex networks," Phys. Rev. E 65, 036104.

Pastor-Satorras, R. and A., Vespignani, 2003, "Epidemics and immunization in scale-free networks," in Handbook of Graphs and Networks: From the Genome to the Internet, edited by S. Bornholdt and H. G. Schuster, (Wiley-VCH, Berlin), p. 111.

Pastor-Satorras, R. and A. Vespignani, 2004, Evolution and Structure of the Internet: A Statistical Physics Approach (Cambridge University Press, Cambridge).

Paul, G., R. Cohen, S. Sreenivasan, S. Havlin, and H. E. Stanley, 2007, "Graph partitioning induced phase transitions," Phys. Rev. Lett. 99, 115701.

Pearl, J., 1988, Probabilistic Reasoning in Intelligent Systems: Networks of Plausible Inference (Morgan Kaufmann, San Francisco).

Pecora, L. M. and T. L. Carroll, 1998, "Master stability functions for synchronized coupled systems," Phys. Rev. Lett. 80, 2109.

Peierls, R., 1936, "On Ising's ferromagnet model," Proc. Cambridge Phil. Soc. 32, 477.

Pȩkalski, A. 2001, "Ising model on a small-world network," 
Phys. Rev. E 64, 057104.

Perkovic, O., K. Dahmen, and J. P. Sethnal, 1995, "Avalanches, Barkhausen noise, and plain old criticality," Phys. Rev. Lett. 75, 4528.

Petermann, T. and P. De Los Rios, 2004, "Role of clustering and gridlike ordering in epidemic spreading," Phys. Rev. E 69, 066116.

Picard, J.-C. and H. Ratliff, 1975, "Minimum cuts and related problems," Networks 5, 357.

Pikovsky, A. S., M. G. Rosenblum, and J. Kurths, 2001, Synchronization: A Universal Concept in Nonlinear Sciences (Cambridge University Press, Cambridge).

Pittel, B., J. Spencer, and N. Wormald, 1996, "Sudden emergence of a giant $k$-core in a random graph," J. Combin. Theor. B 67, 111.

Pretti, M. and A. Pelizzola, 2003, "Stable propagation algorithm for the minimization of the Bethe free energy," J. Phys. A 36, 11201.

Radicchi, F. and H. Meyer-Ortmanns, 2006, "Entrainment of coupled oscillator on regular networks by pacemakers," Phys. Rev. E 73, 036218.

Reichardt, J. and S. Bornholdt, 2004, "Detecting fuzzy community structures in complex networks with a Potts model," Phys. Rev. Lett. 93, 218701.

Reichardt, J. and S. Bornholdt, 2006, "Statistical mechanics of community detection," Phys. Rev. E 74, 016110.

Restrepo, J. G., E. Ott, and B. R. Hunt, 2005, "Onset of synchronization in large networks of coupled oscillators," Phys. Rev. E 71, 036151.

Rizzo, T., B. Wemmenhove, and H. J. Kappen, 2006, "On Cavity approximations for graphical models," eprint condmat/0608312.

Rosvall, M., P. Minnhagen, and K. Sneppen, 2004, "Navigating networks with limited information," Phys. Rev. E 71, 066111.

Roy, S. and S. M. Bhattacharjee, 2006, "Is small-world network disordered?," Phys. Letters A 352, 13.

Rozenfeld, H. D. and D. ben-Avraham, 2007, "Percolation in hierarchical scale-free nets," Phys. Rev. E 75, 061102.

Sade, M., T. Kalisky, S. Havlin, and R. Berkovits, 2005, "Localization transition on complex networks via spectral statistics," Phys. Rev. E 72, 066123.

Sánchez, A. D., J. M. López, and M. A. Rodríguez, 2002, "Nonequilibrium phase transitions in directed small-world networks," Phys. Rev. Lett. 88, 048701.

Scalettar R. T., 1991, "Critical properties of an Ising model with dilute long range interactions," Physica A 170, 282.

Schneider, T. and E. Pytte, 1977, "Random-field instability of the ferromagnetic state," Phys. Rev. B 15, 1519.

Schwartz, N., R. Cohen, D. ben-Avraham, A.-L. Barabási, and S. Havlin, 2002, "Percolation in directed scale-free networks," Phys. Rev. E 66, 015104.

Schwartz, J. M., A. J. Liu, and L. Q. Chayes, 2006, "The onset of jamming as the sudden emergence of an infinite $k$-core cluster," Europhys. Lett. 73, 560566.

Serrano, M. A. and M. Boguñá, 2006a, "Percolation and epidemic thresholds in clustered networks," Phys. Rev. Lett. 97, 088701.

Serrano, M. A. and M. Boguna, 2006b, "Clustering in complex networks. I. General formalism," Phys. Rev. E 74, 056114.

Serrano, M. A. and M. Boguna, 2006c, "Clustering in complex networks. II. Percolation properties," Phys. Rev. E 74, 056115 .

Serrano, M. A. and P. De Los Rios, 2007, "Interfaces and the edge percolation map of random directed networks," eprint arXiv:0706.3156 [cond-mat].

Sethna, J. P., K. Dahmen, S. Kartha, J. A. Krumhansl, B. W. Roberts, and J. D. Shore, 1993, "Hysteresis and hierarchies: Dynamics of disorder-driven first-order phase transformations," Phys. Rev. Lett. 70, 3347.

Sethna, J. P., K. A. Dahmen, and C. R. Myers, 2001, "Crackling noise," Nature 410, 242.

Seyed-allaei, H., G. Bianconi, and M. Marsili, 2006, "Scalefree networks with an exponent less than two," Phys. Rev. E 73, 046113.

Skantzos N. S., I. P. Castillo, and J. P. L. Hatchett, 2005, Cavity approach for real variables on diluted graphs and application to synchronization in small-world lattices, Phys. Rev. E 72, 066127.

Skantzos, N. S. and J. P. L. Hatchett, 2007, "Statics and dynamics of the Lebwohl-Lasher model in the Bethe approximation," Physica A 381, 202.

Soderberg, B., 2002, "A general formalism for inhomogeneous random graphs," Phys. Rev. E 66, 066121.

Solé, R. V. and S. Valverde, 2001, "Information transfer and phase transitions in a model of internet traffic," Physica A 289, 595 .

Solomonoff, R. and A. Rapoport, 1951, "Connectivity of random nets," Bull. Math. Biophys. 13, 107.

Son, S.-W., H. Jeong, and J. D. Noh, 2006, "Random field Ising model and community structure in complex networks," Eur. Phys. J. B 50, 431.

Song, C., S. Havlin, and H. A. Makse, 2005, "Self-similarity of complex networks," Nature 433, 392.

Song, C., S. Havlin, and H. A. Makse, 2006, "Origins of fractality in the growth of complex networks," Nature Physics 2, 275 .

Song, C., L. K. Gallos, S. Havlin, and H. A. Makse, 2007, "How to calculate the fractal dimension of a complex network: the box covering algorithm," J. Stat. Mech. P03006.

Sood, V. and P. Grassberger, 2007, "Localization transition of biased random walks on random networks," Phys. Rev. Lett. 99, 098701.

Sood, V. and S. Redner, 2005, "Voter model on heterogeneous graphs," Phys. Rev. Lett. 94, 178701.

Stosic, T., B. D. Stosic, and I. P. Fittipaldi, 1998, "Exact zerofield susceptibility of the Ising model on a Cayley tree," J. Magn. Magn. Mater. 177, 185.

Strauss D., 1986, "On a general class of models for interaction," SIAM Review 28, 513.

Strogatz, S. H. 2000, "From Kuramoto to Crawford: Exploring the onset of synchronization in populations of coupled oscillators," Physica D 143, 1.

Strogatz, S. H., 2003, Sync: The Emerging Science of Spontaneous Order (Hyperion, New York).

Suchecki, K., V. M. Eguíluz, and M. San Miguel, 2005a, "Conservation laws for the voter model in complex networks," Europhys. Lett. 69, 228.

Suchecki, K., V. M. Eguíluz, and M. San Miguel, 2005b, "Voter model dynamics in complex networks: Role of dimensionality, disorder, and degree distribution," Phys. Rev. E 72, 036132.

Svenson P., 2001, "Freezing in random graph ferromagnets," Phys. Rev. E 64, 036122.

Svenson, P. and M. G. Nordahl, 1999, "Relaxation in graph coloring and satisfiability problem," Phys. Rev. E 59, 3983.

Szabó, G., M. J. Alava, and J. Kertész, 2003, "Structural transitions in scale free networks," Phys. Rev. E 67 67, 
056102.

Tadić B., K. Malarz, and K. Kulakowski, 2005, "Magnetization reversal in spin patterns with complex geometry," Phys. Rev. Lett. 94, 137204.

Tadić, B., G. J. Rodgers, and S. Thurner, 2007, "Transport on complex networks: Flow, jamming and optimization," Int. J. Bifurcation and Chaos 17, 2363.

Tadić, B. and S. Thurner, 2004, "Information super-diffusion on structured networks," Physica A 332, 566.

Tadić, B., S. Thurner, and G. J. Rodgers, 2004, "Traffic on complex networks: Towards understanding global statistical properties from microscopic density fluctuations," Phys. Rev. E 69, 036102.

Tanaka, K. 2002, "Statistical-mechanical approach to image processing," J. Phys. A 35, R81.

Tanaka, H.-A., A. J. Lichtenberg, and S. Oishi, 1997, "First order phase transition resulting from finite inertia in coupled oscillator systems," Phys. Rev. Lett. 78, 2104.

Tang, M., Z. Liu, and J. Zhou, 2006, "Condensation in a zero range process on weighted scale-free networks," Phys. Rev. E 74, 036101.

Thouless, D. J., 1986, "Spin-Glass on a Bethe Lattice," Phys. Rev. Lett. 56, 1082.

Thouless, D. J., P. W. Anderson, and R. G. Palmer, 1977, "Solution of solvable model of a spin glass," Philos. Mag. 35, 593.

Timme M., 2006, "Does dynamics reflect topology in directed networks?," Europhys. Lett. 76, 367.

Timme, M., F. Wolf, and T. Geisel, 2004, "Topological speed limits to network synchronization," Phys. Rev. Lett. 92, 074101.

Toroczkai, Z. and K. E. Bassler, 2004, "Jamming is limited in scale-free systems," Nature 428, 716.

Vazquez A., 2006a, "Polynomial growth in branching processes with diverging reproductive number," Phys. Rev. Lett. 96, 038702.

Vazquez A., 2006b, "Spreading dynamics on small-world networks with connectivity fluctuations and correlations," eprint q-bio/0603010.

Vázquez, A. and Y. Moreno, 2003, "Resilience to damage of graphs with degree correlations," Phys. Rev. E 67, 015101 (R).

Vázquez, A. and M. Weigt, 2003, "Computational complexity arising from degree correlations in networks," Phys. Rev. E 67, 027101.

Viana, L. and A. J. Bray, 1985, "Phase diagrams for dilute spin glasses," J. Phys. C 18, 3037.

Vilone, D. and C. Castellano, 2004, "Solution of voter model dynamics on annealed small-world networks," Phys. Rev. E 69, 016109 .

Waclaw, B., L. Bogacz, Z. Burda, and W. Janke, 2007, "Condensation in zero-range processes on inhomogeneous networks," Phys. Rev. E 76, 046114.

Waclaw, B. and I. M. Sokolov, 2007, "Finite size effects in Barabási-Albert growing networks," Phys. Rev. E 75, 056114 .

Walsh T., 1999, "Search in a small world," in Proceedings of the 16the International Joint Conference on Artificial Intelligence, edited by T. Dean (Morgan Kaufmann, San Francisco, CA), p. 1172.

Wang, X. F. and G. Chen, 2002, "Synchronization in smallworld dynamical networks," Int. J. Bifurcation Chaos Appl. Sci. Eng. 12, 187.

Wang, W.-X., B.-H. Wang, C.-Y. Yin, Y.-B. Xie, and T. Zhou,
2006, "Traffic dynamics based on local routing protocol on a scale-free network," Phys. Rev. E 73, 026111.

Warren, C. P., L. M. Sander, and I. M. Sokolov, 2003, "Epidemics, disorder, and percolation," Physica A 325, 1.

Watts, D. J., 1999, Small Worlds: The Dynamics of Networks between Order and Randomness (Princeton University Press, Princeton, NJ).

Watts, D. J., 2002, "A simple model of global cascades on random networks," PNAS 99, 5766.

Watts, D. J., P. S. Dodds, and M. E. J. Newman, 2002, "Identity and search in social networks," Science 296, 1302.

Watts, D. J. and S. H. Strogatz, 1998, "Collective dynamics of 'small-world' networks," Nature 393, 440.

Weigt, M. and A. K. Hartmann, 2000, "Number of guards needed by a museum: A phase transition in vertex covering of random graphs," Phys. Rev. Lett. 84, 6118.

Weigt, M. and A. K. Hartmann, 2001, "Minimal vertex covers on finite-connectivity random graphs: A hard-sphere lattice-gas picture," Phys. Rev. E 63, 056127.

Weigt, M. and H. Zhou, 2006, "Message passing for vertex covers," Phys. Rev. E 74, 046110.

Willinger, W., R. Govindan, S. Jamin, V. Paxson, and S. Shenker, 2002, "Scaling phenomena in the Internet: Critically examining criticality," PNAS 99, 2573.

Wołoszyn, M., D. Stauffer, and K. Kulakowski, 2007, "Orderdisorder phase transition in a cliquey social network," Eur. Phys. J. B 57, 331.

Wu, F. Y., 1982, "The Potts model," Rev. Mod. Phys. 54, 235.

Wu, Z., L. A. Braunstein, V. Colizza, R. Cohen, S. Havlin, and H. E. Stanley, 2006, "Optimal paths in complex networks with correlated weights: The worldwide airport network," Phys. Rev. E 74, 056104.

$\mathrm{Wu}$, F. and B. A. Huberman, 2004, "Social structure and opinion formation," eprint cond-mat/0407252.

Wu, Z., C. Lagorio, L. A. Braunstein, R. Cohen, S. Havlin, and H. E. Stanley, 2007a, "Numerical evaluation of the upper critical dimension of percolation in scale-free networks," Phys. Rev. E 75, 066110.

Wu, A.-C., X.-J. Xu, and Y.-H. Wang, 2007b, "Excitable Greenberg-Hastings cellular automaton model on scale-free networks," Phys. Rev. E 75, 032901.

Yamada, H. 2002, "Phase-locked and phase drift solutions of phase oscillators with asymmetric coupling strengths," Progr. Theor. Phys. 108, 13.

Yedidia, J. S., W. T. Freeman, and Y. Weiss, 2001, in $A d$ vances in Neural Information Processing Systems, edited by T. K. Leen, T. G. Dietterich, and V. Tresp, (MA: MIT Press, Cambridge), "Generalized belief propagation," 13, pp. 689-695.

Zanette, D. H., 2007, "Coevolution of agents and networks in an epidemiological model," eprint 0707.1249 [cond-mat].

Zdeborová, L. and F. Krząkała, 2007, "Phase transitions in the coloring of random graphs," Phys. Rev. E 76, 031131.

Zhang, H., Z. Liu, M. Tang, and P. M. Hui, 2007, "An adaptive routing strategy for packet delivery in complex networks," Phys. Lett. A 364, 177.

Zhang, X. and M. A. Novotny, 2006, "Critical behavior of Ising models with random long-range (small-world) interactions," Brazilian J. Phys. 36, 664.

Zhou, H. 2003, "Vertex cover problem studied by cavity method: Analytics and population dynamics," Eur. Phys. J. B 32, 265.

Zhou, H. 2005, "Long range frustrations in a spin glass model 
of the vertex cover problem ," Phys. Rev. Lett. 94, 217203.

Zhou, H. and R. Lipowsky, 2005, "Dynamic pattern evolution on scale-free networks," PNAS 102, 10052.

Zhou, S. and R. J. Mondragón, 2004, "The rich-club phenomenon in the Internet topology," IEEE Commun. Lett. 8, 180 .

Zhou, C., A. E. Motter, and J. Kurths, 2006, "Universality in the synchronization of weighted random networks," Phys. Rev. Lett. 96, 034101.

Zimmermann, M. G., Eguíluz V. M., and M. San Miguel, 2004, "Coevolution of dynamical states and interactions in dynamic networks," Phys. Rev. E 69, 065102. 